\title{
Asymptotic Approximations for the Sound Generated by Aerofoils in Unsteady Subsonic Flows
}

\author{
Lorna Jane Ayton \\ Churchill College
}

Department of Applied Mathematics and Theoretical Physics

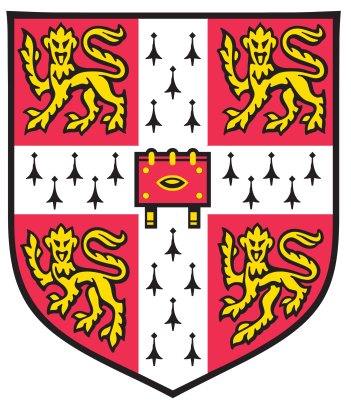

A Dissertation SUbmitted For THE DeGree of Doctor of Philosophy AT The University of CAMbridge SEPTEMBER 2014 



\section{Asymptotic Approximations for the Sound Generated by Aerofoils in Unsteady Subsonic Flows}

\section{Lorna Jane Ayton}

This thesis considers the sound generated by unsteady perturbations interacting with solid aerofoils in background steady flows, in an attempt to further develop analytic models for the noise generated by blades within turboengines. Specifically, high-frequency unsteady gust and sound wave perturbations are considered and asymptotic results are obtained for, primarily, the far-field noise.

Previous analytic work has examined high-frequency gust-aerofoil interactions in steady uniform flows using rapid distortion theory, and has focused on aerofoils with simple geometries. We extend this to deal with aerofoils with more realistic geometries (by including camber, thickness, and angle of attack), as well as considering the new topic of sound-aerofoil interactions in steady uniform flows for aerofoils with realistic geometries. The assumption of a steady uniform flow is later relaxed and we investigate the sound generated by high-frequency gust-aerofoil interactions in steady shear flows.

Throughout all of the aforementioned work, the key process involves identifying various asymptotic regions around the aerofoil where different sources dominate the generation of sound. Solutions are obtained in each region and matched using the asymptotic matching rule. The dominant regions producing noise are the local, "inner", regions at the leading and trailing edges of the aerofoil. Approximations for the far-field noise (in the "outer" regions) are the principal results, however one can also extract approximations for the unsteady pressure generated on the surface of the aerofoil.

The surface pressure generated by high-frequency gust-aerofoil interaction in uniform flow is found to contain a singularity at the leading-edge stagnation point, thus the final piece of work in this thesis focuses more closely on turbulent interactions with solid body stagnation points in uniform flow, eliminating this singularity. 



\section{Declaration}

This thesis describes research carried out in the Department of Applied Mathematics and Theoretical Physics of Cambridge University. This is a result of my own work and includes nothing which is the outcome of work done in collaboration except where specifically indicated in the text. The analysis in Chapter 1 has been published as Ayton \& Peake (2013), and Chapter 3 has been submitted for publication as Ayton \& Peake $(2014 b)$. Parts of Chapter 3 have been presented at an international conference as Ayton \& Peake (2014a). Some elements of the introduction have been taken from Ayton \& Peake (2013) and Ayton \& Peake (2014b). No part of the work contained herein has been submitted to any other university or place of learning for any degree, diploma or other qualification.

Lorna J. Ayton

September 2014 


\section{Acknowledgements}

I would first like to thank my supervisor, Prof. Nigel Peake, for his guidance and support during the past three years, and for his great patience and calm frame of mind during all of the problems I have encountered with my research. I must also thank my office-mate, Alison Ming, without whom less tea would have been drunk and fewer crosswords would have been completed. More importantly, without her I would have never obtained the minimal amount of Linux knowledge required to survive in the Department. I would further like to thank past and present members of the Waves Group; Ed, Conor and Justin, for always being willing to spend their time helping and guiding me in the world of research.

I'm endlessly grateful for the support of my family and friends; Mum, who doesn't understand any of the work that I do but does not let that hinder her encouragement and pride; Dad, who is always there to give good advice at any hour of the day or night; Ryan, who tries to not let me stress out too much and patiently proof-reads anything I give him; and Laura and Pete, the friends who are always there (with wine) when things go wrong.

This work was supported by an EPSRC CASE grant in conjunction with Rolls-Royce PLC, and undertaken at DAMTP, all of whom I would like to thank.

Finally, a great deal of gratitude must go to Churchill College, who have given me a happy home for seven years, as well as financial support for various conferences. 
For Grandad,

I wish you could see this. 


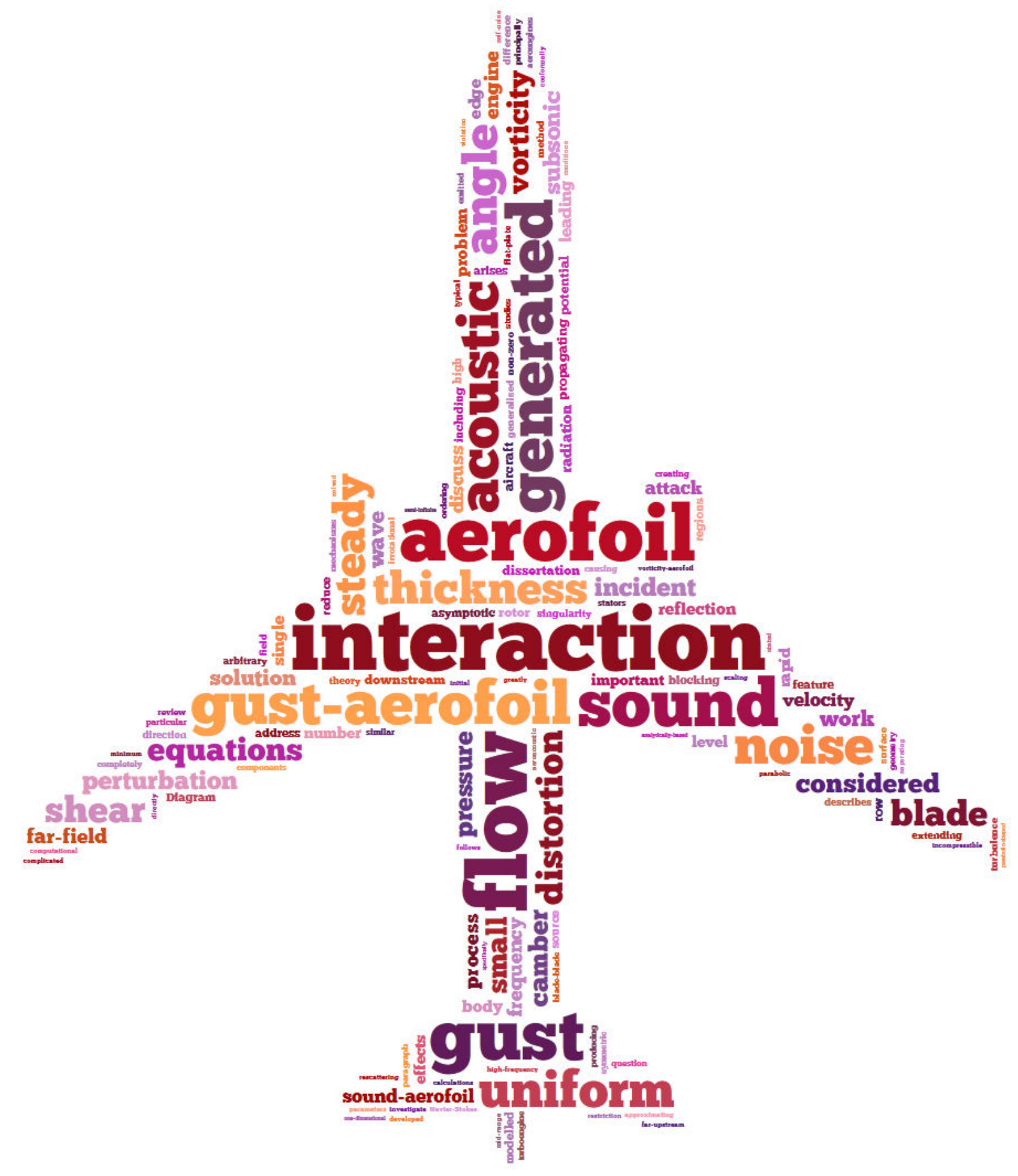




\section{Contents}

List of Figures $\quad 4$

$\begin{array}{ll}\text { Introduction } & 7\end{array}$

1 High-Frequency Sound Generated by Sound-Aerofoil Interaction in Subsonic Uniform Flow 16

1.1 Formulation of the Problem . . . . . . . . . . . . . . . . 17

1.1.1 Aerofoil Geometry and Steady Mean Flow . . . . . . . . . . . . . 17

1.1.2 Unsteady Disturbance Equations . . . . . . . . . . . . . . . . 19

1.1.3 Form of the Incident Sound . . . . . . . . . . . . . . . . . . . . . 21

1.2 Leading-Edge Inner Solution . . . . . . . . . . . . . . . . . . . . . 24

1.2.1 Solution for the Flat-Plate Term $H_{0} \ldots \ldots$. . . . . . . . . . 25

1.2.2 Solution for the Thickness-Related Term $H_{1}$. . . . . . . . . . . . 28

1.2.3 Solution for the Thickness-Related Term $H_{2}$. . . . . . . . . . . . 31

1.2.4 Solution for the Thickness-Related Term $H_{3}$. . . . . . . . . . . . 33

1.2.5 Solution for the Camber-Related Terms $P_{i}, i=1,2,3 \ldots$. . . . . 35

1.3 Leading-Edge Outer and Transition Solutions . . . . . . . . . . . . . 36

1.3.1 Leading-Edge Ray Field . . . . . . . . . . . . . . . . . . 37

1.3.2 Leading-Edge Transition Solution . . . . . . . . . . . . . . 38

1.4 Trailing-Edge Solutions . . . . . . . . . . . . . . . . . . . . . 42

1.4.1 Trailing-Edge Ray Field . . . . . . . . . . . . . . . . . . . . . . . 42

1.4.2 Trailing-Edge Inner Solution . . . . . . . . . . . . . . . . . . . . 43

1.4.3 Trailing-Edge Transition Solution . . . . . . . . . . . . . . . 46

1.4.4 Matching the Trailing-Edge Transition and Inner Solutions . . . . 47

1.4.5 Far-Field Solution from the Trailing Edge . . . . . . . . . . . . . 48

1.5 Total Far-Field Solution . . . . . . . . . . . . . . . . . . . . 50

1.6 Results and Discussion . . . . . . . . . . . . . . . . . . 51

1.6.1 Far-Field Pressure Directivity for the Scattered Sound . . . . . . 51

1.6.2 Total Far-Field Pressure . . . . . . . . . . . . . . . . . . . 58

1.7 Conclusions . . . . . . . . . . . . . . . . . 61

$\begin{array}{ll}\text { List of Symbols for Chapter } 1 & 63\end{array}$

$\begin{array}{ll}\text { Appendices } & 66\end{array}$ 
2 High-Frequency Sound Generated by Gust-Aerofoil Interaction in Subsonic Uniform Flow $\quad 73$

2.1 Formulation of the Governing Equations . . . . . . . . . . . . . . 75

2.2 Leading-Edge Inner Solution . . . . . . . . . . . . . . . . . . 78

2.2.1 Solution for the Flat-Plate Term $H_{0} \ldots \ldots \ldots$. . . . . . . . . 79

2.2.2 Solution for Thickness-Related Term $H_{1}$. . . . . . . . . . . . . . 79

2.2.3 Solution for Thickness-Related Term $H_{2}$. . . . . . . . . . . . . . 80

2.2.4 Solution for Thickness-Related Term $H_{3}$. . . . . . . . . . . . . . 82

2.2.5 Solution for Camber-Related Terms $P_{i}, i=1,2,3 \ldots \ldots$. . . . 83

2.3 Outer Solutions . . . . . . . . . . . . . . . . . . . . . . 83

2.3.1 Leading-Edge Acoustic Outer Solution . . . . . . . . . . . . 85

2.3.2 Trailing-Edge Acoustic Outer Solution . . . . . . . . . . . . . 86

2.4 Leading-Edge Transition Solution . . . . . . . . . . . . . . . . . . 86

2.5 Trailing-Edge Inner Solution . . . . . . . . . . . . . . . . . . . 88

2.6 Trailing-Edge Transition Solution . . . . . . . . . . . . . . . . 89

2.7 Total Far-Field Solution _ . . . . . . . . . . . . . . . . . . . . . . . 90

2.8 Results . . . . . . . . . . . . . . . . . . . . . . . . . . . 92

2.8.1 Far-Field Acoustic Pressure . . . . . . . . . . . . . . . . . . 93

2.8.2 Unsteady Surface Pressure . . . . . . . . . . . . . . . . . 97

2.8.3 Comparison with Numerical Results . . . . . . . . . . . . . . 101

2.9 Conclusions . . . . . . . . . . . . . . . . . . . . . . . . . . . . 104

$\begin{array}{ll}\text { List of New Symbols for Chapter } 2 & 108\end{array}$

$\begin{array}{ll}\text { Appendices } & 109\end{array}$

3 High-Frequency Sound Generated by Gust-Aerofoil Interaction in $\begin{array}{lr}\text { Subsonic Shear Flow } & 119\end{array}$

3.1 Formulation and Governing Equations . . . . . . . . . . . . . . . . . 122

3.2 Form of the Incident Gust . . . . . . . . . . . . . . . . . . . . . . 125

3.3 Leading-Edge Inner Solution . . . . . . . . . . . . . . . 127

3.3.1 General Solution for Leading-Edge Inner Acoustic Pressure . . . . 128

3.3.2 Outer limit of the inner solution . . . . . . . . . . . . . . . . 130

3.4 Leading-Edge Outer Solution _. . . . . . . . . . . . . . . . . 134

3.5 Leading-Edge Transition Solution . . . . . . . . . . . . . . . . 137

3.6 Trailing-Edge Solutions . . . . . . . . . . . . . . . . . . . . . . . . . 138

3.6.1 Trailing-Edge Inner Solution . . . . . . . . . . . . . . . 138 
3.6.2 Trailing-Edge Outer Solution _. . . . . . . . . . . . . . 141

3.6.3 Trailing-Edge Transition Solution . . . . . . . . . . . . . . . . 141

3.7 Total far-field solution . . . . . . . . . . . . . . . . . . . . . 142

3.8 Results . . . . . . . . . . . . . . . . . . . 143

3.8.1 Evaluation of the Streamfunction . . . . . . . . . . . . . . . 144

3.8.2 Far-Field Results . . . . . . . . . . . . . . . . . . . . . . . 145

3.9 Conclusions . . . . . . . . . . . . . . . . . . . . 152

List of Symbols for Chapter $3 \quad 155$

$\begin{array}{lr}\text { Appendices } & 157\end{array}$

4 Leading-Edge Stagnation-Point Noise Generated by Turbulence in Subsonic Uniform Flow 161

4.1 Streamfunction and Drift Function . . . . . . . . . . . . . . . . 164

4.1 .1 Streamfunction . . . . . . . . . . . . . . . . 164

4.1 .2 Joukowski Transformation of Coordinates . . . . . . . . . . 165

4.1 .3 Drift Function . . . . . . . . . . . . . . . . . 166

4.2 Velocity Potential Given an Incident Gust . . . . . . . . . . . . . . . . 168

4.2.1 High-Frequency Solution . . . . . . . . . . . . . . . . . . . 169

4.2 .2 Low-Frequency Solution . . . . . . . . . . . . . . . . . . . 174

4.3 Effect of Turbulence . . . . . . . . . . . . . . . . . . . 175

4.3.1 High-Frequency Pressure Spectra in the Far Field . . . . . . . . . 177

4.3.2 High- and Low-Frequency Surface Pressure Spectrum . . . . . . . 177

4.4 Results . . . . . . . . . . . . . . . . . . . . . . . 180

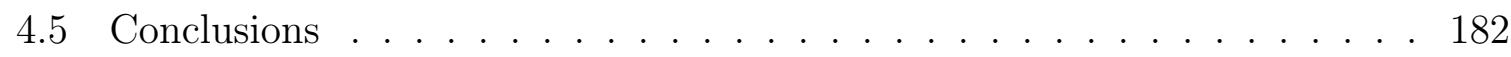

List of Symbols for Chapter 4

$\begin{array}{lr}\text { Appendices } & 186\end{array}$

5 Concluding Remarks and Further Work 193

$\begin{array}{ll}\text { Bibliography } & 197\end{array}$ 


\section{List of Figures}

0.1 Aeroengine noise reduction over time due to improving designs . . . . . 8

0.2 Change over time in the region around Frankfurt Airport in which aircraft take-off noise equals approximately $85 \mathrm{~dB} \ldots \ldots$. . . . . . . 8

0.3 Relative contributions to the total engine noise from different components in early and modern turbofans. . . . . . . . . . . . . . . . . . . 99 9

0.4 Diagram of a Rolls-Royce turboengine attached to a Boeing 757 . . . . . 9

0.5 Schematic of key features that generate noise within an aeroengine. . . . 10

0.6 Two-dimensional blade-blade interaction model . . . . . . . . . . . . . . 10

0.7 Diagram of a generalised aerofoil with camber, thickness and angle of attack 11

1.1 Asymptotic regions around the aerofoil in both physical and $(\phi, \psi)$-space 23

1.2 Steepest descents contour for phase function $-a(\lambda)$ in the $\lambda$-plane. . . . . 27

1.3 Location of the branch cut of $\sqrt{v-\beta \mathrm{e}^{-3 \pi \mathrm{i} / 4}}$ in the $v$-plane, and contour

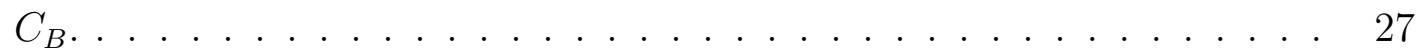

1.4 Comparison of real and imaginary parts of the uniformly-valid and non uniformly-valid far-field approximations for the directivity of $H_{1} \ldots$. . .

1.5 Magnitude of the far-field limit of the leading-edge transition solution for a NACA 1112 aerofoil with $k=10$. . . . . . . . . . . . . . . . . . 41

1.6 Far-field scattered pressure directivity for a NACA 1112 aerofoil, $k=6$, $\alpha_{i}=0^{\circ}, \chi=45^{\circ}, k w=10$. The Mach number is varied from 0.6 to 0.8 . .

1.7 Far-field scattered pressure directivity for a NACA 4-digit aerofoil with $10 \%$ maximum camber at $10 \%$ chord length, $k=6, \alpha_{i}=0^{\circ}, \chi=45^{\circ}$, $k w=10, M_{\infty}=0.7$. The thickness ratio is varied from $0 \%$ to $12 \%$. . .

1.8 Far-field scattered pressure directivity for a NACA 1112 aerofoil with $k=$ $6, \chi=45^{\circ}, k w=10, M_{\infty}=0.7$. The angle of attack is varied from $0^{\circ}$ to $10^{\circ} \ldots \ldots \ldots \ldots \ldots \ldots \ldots$

1.9 Far-field scattered pressure directivity for a NACA 1112 aerofoil with $k=$ $6, \alpha_{i}=0^{\circ}, k w=10, M_{\infty}=0.7$. The angle of the incident sound wave, $\chi$, is varied from $30^{\circ}$ to $60^{\circ} \ldots \ldots \ldots \ldots \ldots$

1.10 Far-field scattered pressure directivity for a NACA 1112 aerofoil with $k=$ $6, \alpha_{i}=0^{\circ}, \chi=45^{\circ}, M_{\infty}=0.7$. The acoustic frequency, $k w$, is varied from 8 to 12 . 
1.11 Far-field scattered pressure directivity for a NACA 4-digit aerofoil with maximum camber at $10 \%$ chord length, and $12 \%$ thickness, $k=6, k w=$ $10, \alpha_{i}=0^{\circ}, \chi=135^{\circ}, M_{\infty}=0.7$. The percentage of maximum camber is varied from $1 \%$ to $9 \%$.

1.12 Maximum and minimum total far-field pressure for uncambered NACA 4-digit aerofoils, as functions of angle of attack, for various thickness parameters. . . . . . . . . . . . . . . . . . .

1.13 Maximum and minimum total far-field pressure, for NACA 4-digit aerofoils with maximum camber at $10 \%$ chord and zero angle of attack, as functions of maximum \% camber, for various thickness parameters.

2.1 Asymptotic regions around the aerofoil in both physical and $(\phi, \psi)$-space

2.2 Far-field scattered pressure directivity for a NACA 4-digit aerofoil with $1 \%$ maximum camber at $10 \%$ chord length, $k=8, \alpha_{i}=0^{\circ}, M_{\infty}=0.7$, $\theta_{g}=45^{\circ}, k_{3}=0$. The thickness ratio is varied from $0 \%$ (flat plate) to $12 \% .92$

2.3 Far-field scattered pressure directivity for a $10 \%$ thick Joukowski aerofoil with $k=5, \alpha_{i}=0^{\circ}, M_{\infty}=0.6, k_{3}=0$, and $\theta_{g}=45^{\circ}$. Maximum camber is varied from $0 \%$ to $2 \%$, and the location of maximum camber is at $10 \%$ chord length.

2.4 Far-field scattered pressure directivity for a 6-33 cambered aerofoil with $k=8, \alpha_{i}=0^{\circ}, M_{\infty}=0.5, k_{3}=0$, and $\theta_{g}=60^{\circ}$. Thickness is varied from $0 \%$ to $10 \%$.

2.5 Far-field scattered pressure directivity for a NACA 1112 aerofoil with $k=$ $8, \alpha_{i}=0^{\circ}, M_{\infty}=0.7, k_{3}=0$. The gust angle is varied from $30^{\circ}$ to $60^{\circ}$.

2.6 Far-field scattered pressure directivity for a NACA 1112 aerofoil with $k=$ $8, \alpha_{i}=0^{\circ}, M_{\infty}=0.7, \theta_{g}=45^{\circ} . k_{3}$ is varied from 0 to 0.5 .

2.7 Absolute value of the unsteady surface pressure jump across a NACA aerofoil, with $\theta_{g}=45^{\circ}, k_{3}=0$, and $M=0.6 \ldots \ldots$. . . . .

2.8 Unsteady lift on a NACA 0012 and 1112 aerofoils, with $\theta_{g}=45^{\circ}, k_{3}=0$ and $\alpha_{i}=0^{\circ} . k$ varies from 8 to 20 .

2.9 Comparison of asymptotic and numeric results for a NACA 0012 aerofoil at $M=0.5, k=5 / \beta_{\infty}, k_{t}=1, \alpha_{i}=0,, k_{3}=0$, and $\theta_{g}=45^{\circ} \ldots \ldots 103$

2.10 Unsteady RMS surface pressure on a symmetric Joukowski aerofoil of $12 \%$ thickness, at $M_{\infty}=0.5, k=3 / \beta_{\infty}, k_{3}=0$, and $\theta_{g}=45^{\circ}$.

2.11 Comparison of asymptotic and Amiet far-field pressure directivities for a flat plate with $\theta_{g}=45^{\circ}, k_{t}=1, k_{3}=0$, and $\alpha_{i}=0^{\circ} \ldots \ldots \ldots$ 
2.12 Comparison of asymptotic and numeric far-field pressure directivities for a NACA 0012 aerofoil with $M=0.6, \theta_{g}=45^{\circ}, k_{t}=1, k_{3}=0$, and $\alpha_{i}=0^{\circ} .106$

3.1 Asymptotic regions around the aerofoil in both physical and $(\phi, \psi)$-space. 121

3.2 Leading-edge far-field acoustic pressure for a flat plate . . . . . . . . . 147

3.3 Magnitude of the ray tube scaling factor for various shear flow profiles. $\quad 147$

3.4 Leading-edge far-field acoustic pressure directivity with $k=10, M_{0}=0.2$, $M_{\infty}=0.3$, and $S=0.33$, for Joukowski aerofoils of varying thickness. . . 148

3.5 Trailing-edge far-field pressure directivity with $S=0.333, k=10, \epsilon=0.1$. 148

3.6 Far-field pressure directivity at $r=25$ in almost uniform Gaussian shear flow with $M \approx 0.3, k=10$, and $\epsilon=0 \ldots \ldots$. . . . . . . . 150

3.7 Far-field pressure directivity at $r=25$ for jet-like and wake-like shear flows, with $k=10$, and $\epsilon=0 . \ldots \ldots$. . . . . . . . . 150

3.8 Far-field pressure directivity at $r=25$ with $M_{0}=0.2, M_{\infty}=0.3, k=10$, and varying thickness. . . . . . . . . . . . . . . . . 150

3.9 Far-field pressure directivity at $r=25$ with $M_{0}=0.2, k=10, \epsilon=0.1$ and varying $S \ldots \ldots \ldots \ldots \ldots \ldots \ldots \ldots$

3.10 Far-field pressure directivity for gust-aerofoil interaction in uniform flow with $M=0.6$ around a Joukowski aerofoil of thickness 0.1 , and frequency (as defined in Chapter 2) 10 . . . . . . . . . . . . . . . . . . 152

4.1 Diagram of the flow problem and coordinate system. . . . . . . . . . 167

4.2 Far-field scattered pressure directivity for a NACA 0006 aerofoil, $k=$ $8, \alpha_{i}=0^{\circ}, M_{\infty}=0.6, \theta_{g}=0.01^{\circ}, k_{3}=0$, variables as defined for Chapter 2. 174

4.3 Plot of $h\left(\theta, k_{1} l\right)$ governing the turbulent pressure spectrum far from the ellipse, for varying values of $\theta$. . . . . . . . . . . . . . . . . 180

4.4 Asymptotically obtained cross-PSD close to $\theta=0$ with $U=30, b=2$, $\rho_{0}=1, l=0.0078$, and $M=0.09$, compared to experimental data, on the surface of a NACA 0015 aerofoil.

4.5 Sound pressure levels in the far field from the leading edge of an SD7003 aerofoil measured experimentally, compared to the asymptotically obtained turbulent pressure spectrum. . . . . . . . . . . . . . 


\section{Introduction}

The problem of sound generation and scattering by bodies in flow arises in a number of engineering applications, in particular the important question of predicting the level of far-field noise emitted by aircraft engines. It is important to have a handle on the noise currently emitted from aeroengines because this noise level must be reduced by $50 \%$ by 2020 to comply with the aim set in 2001 by the Advisory Council for Aeronautics Research in Europe. Figures 0.1 and 0.2 illustrate the progress in noise reduction; Figure 0.1 shows the reduction in noise due to improvements in turboengine design, whilst Figure 0.2 shows how the region around an airport where the take-off noise equals approximately 85 decibels has been decreased due to noise reduction efforts. The principal source of noise emitted by an aircraft is from the engine, and Figure 0.3 shows the relative contributions to the total engine noise from the key noise generating features. Early and modern turbofans are compared, showing a very notable reduction in jet noise, but the remaining contributions have not been reduced as significantly. Figure 0.4 shows a typical turboengine found on a large passenger aircraft, and Figure 0.5 illustrates the important components inside the engine where the high levels of noise shown in Figure 0.3 are produced.

Peake \& Parry (2012) give a recent review of the different mechanisms generating noise within the engine, including perhaps principally the jet and (the subject of this thesis) the interaction of the vortical wakes shed from a rotor blade row with a downstream stator blade row; a simplified view of this second process can be seen in Figure 0.6. This "blade-blade" interaction noise contributes to both the fan and the compressor noise seen in Figure 0.5. It is commonly assumed that the background steady flow, to which the vortical disturbances are seen as small perturbations, is uniform (which is the background flow illustrated in Figure 0.6). However, there are a number of situations in which significant upstream mean shear is present. One common example of this is the case in which a rotor operates in shear flow due to incidence or to installation effects (perhaps caused by the wake of some structural element upstream). In this dissertation we therefore discuss blade-blade interactions occurring both in uniform and sheared background steady flows.

The process of sound generation by blade rows in uniform steady flow has been modelled in a number of studies of unsteady vorticity-aerofoil interaction (known also as gust-aerofoil interaction), as will be discussed in the next paragraph. Once the noise has been generated by this gust-aerofoil interaction, it is scattered by the rotating blade row 


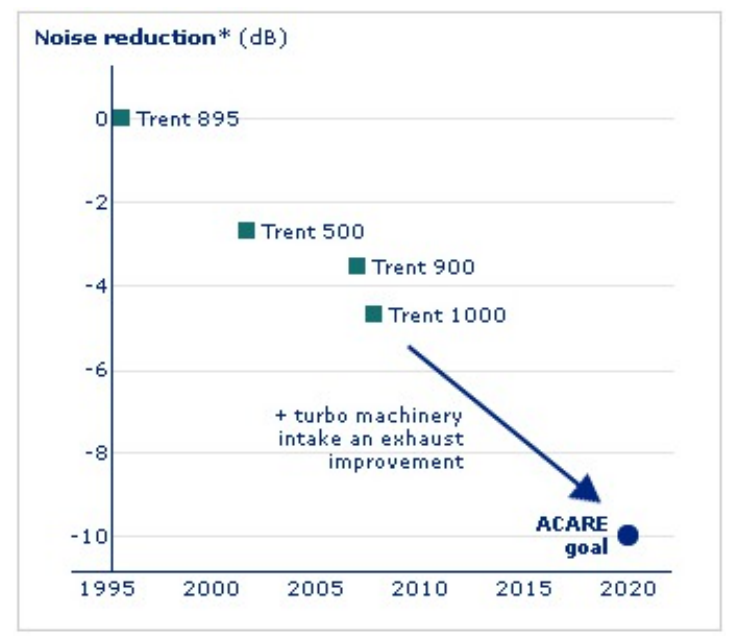

*Corrected for aircraft weight

Figure 0.1: Aeroengine noise reduction over time due to improving designs. Source: Rolls-Royce.

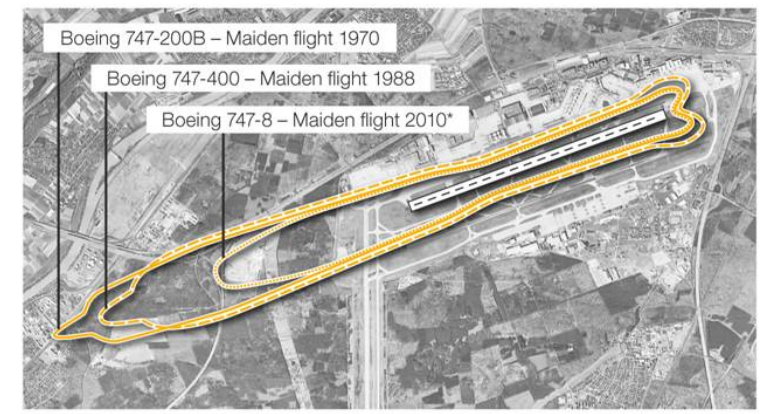

Figure 0.2: Change over time in the region around Frankfurt Airport in which aircraft take-off noise equals approximately $85 \mathrm{~dB}$. Source: Boeing, Lufthansa.

upstream (sound-aerofoil interaction) before being partially radiated from the engine in the forward arc, and the level of so-called rotor blockage is then crucial. The radiated sound may be scattered by the wings, and novel aircraft designs have been proposed in which the engine is mounted above the wing so as to reduce noise levels on the ground via shielding - see Agarwal et al. (2007). In this dissertation we therefore address the question of sound-aerofoil interaction (Chapter 1) and gust-aerofoil interaction (Chapter 2) in background subsonic uniform flows for generalised aerofoils with small thickness, camber and angle of attack. We also consider gust-aerofoil interaction in background shear flows (Chapter 3) for symmetric aerofoils with small thickness. A schematic diagram of a generalised aerofoil (i.e. one with thickness, camber and angle of attack) is given in Figure 0.7.

Unsteady gust-aerofoil interaction (by which we mean a gust interacting with a single aerofoil) in uniform flow has received a great deal of attention, including Sears' famous result (Sears, 1941) concerning the fluctuating lift on a flat-plate aerofoil interacting with a sinusoidal gust in an incompressible flow (see Goldstein (1976) for details, and other early work). Extension of Sears' result to include the effects of (thin) aerofoil geometry has been completed by Goldstein \& Atassi (1976) and Atassi (1984). A number of approaches have been based on the version of rapid distortion theory devised by Goldstein (1978b), in which the linearised unsteady velocity perturbation to the steady (potential) base flow is decomposed into vortical and irrotational parts, yielding two equations: one 


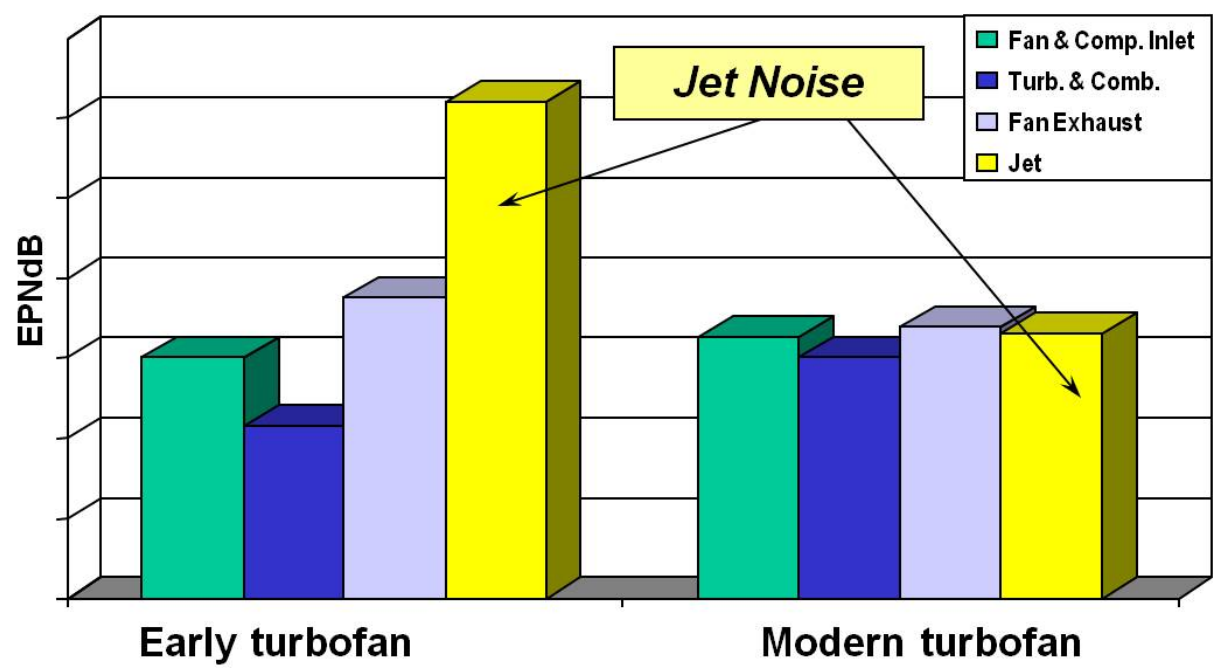

Figure 0.3: Relative contributions to the total engine noise from different components in early and modern turbofans. Source; Peake \& Parry (2012), with permission.

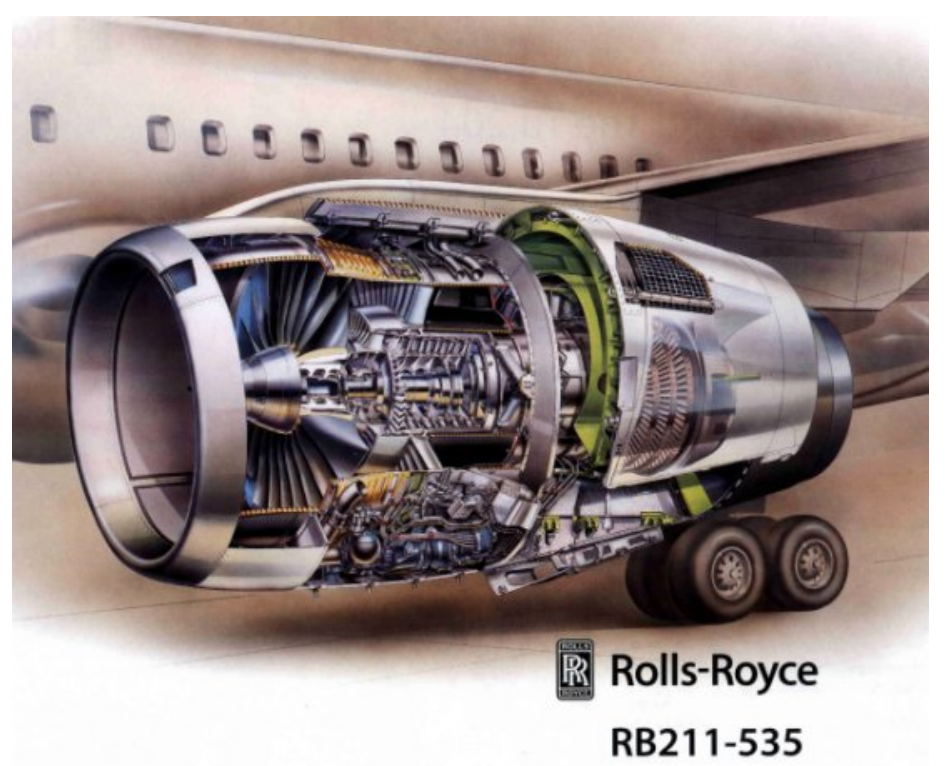

Figure 0.4: Diagram of a Rolls-Royce turboengine attached to a Boeing 757. 


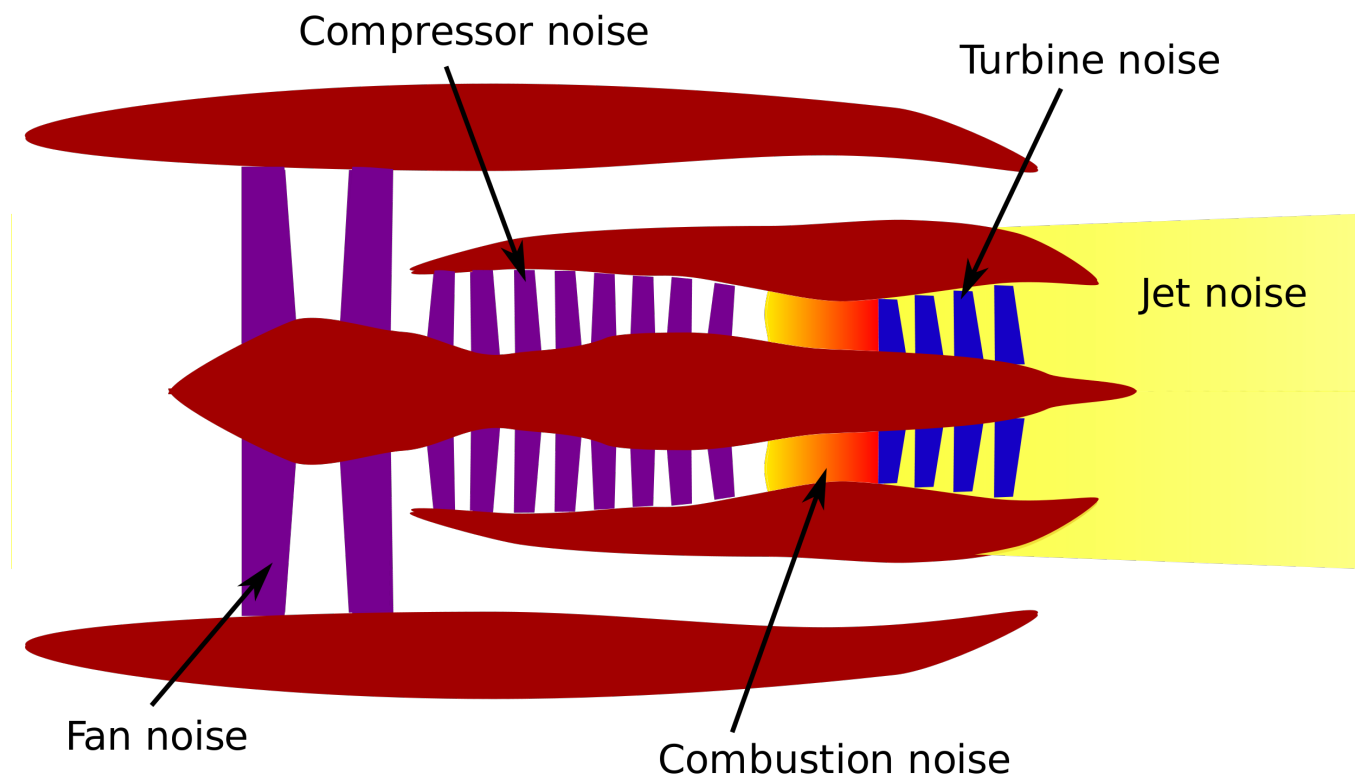

Figure 0.5: Schematic of key features that generate noise within an aeroengine.
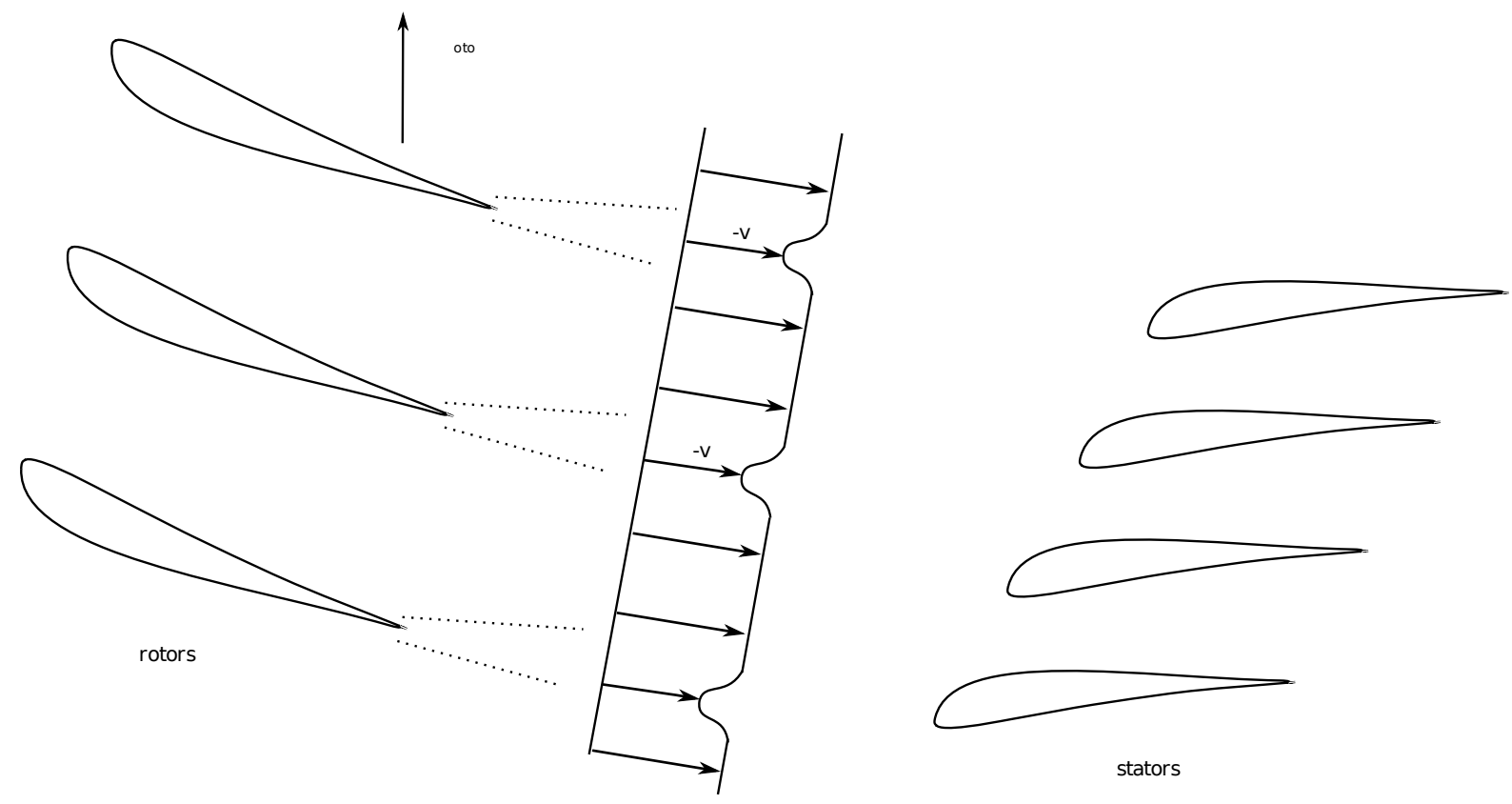

Figure 0.6: Two-dimensional blade-blade interaction model; the blades are unrolled onto two staggered infinite cascades. The rotors translate at constant velocity $U_{\text {rotor }}$ whilst the stators are stationary. Far upstream (to the left), the background flow is uniform, with velocity $U$. The unsteady perturbation generated by the rotor wakes is illustrated by velocity $v$. 


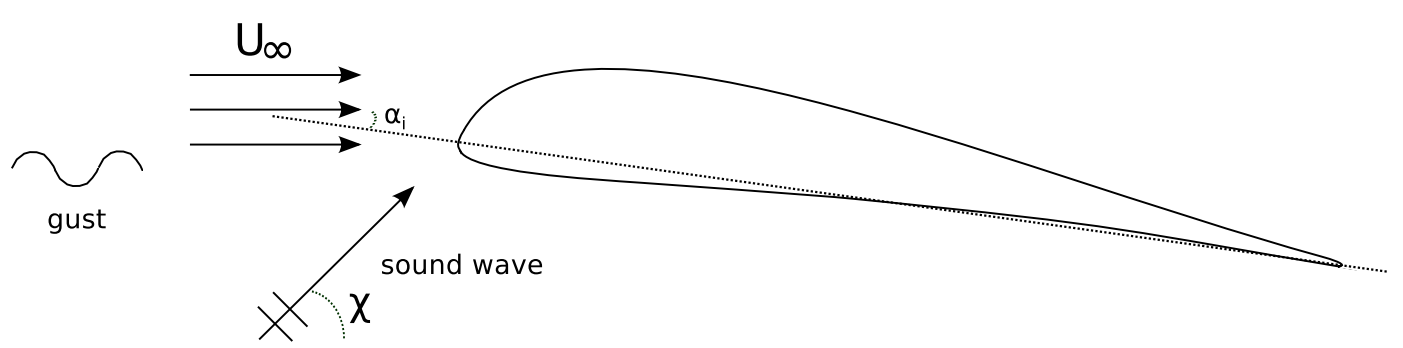

Figure 0.7: Diagram of a generalised aerofoil, with camber, thickness and angle of attack, $\alpha_{i}$, with respect to the direction of the background steady flow. The upstream perturbation is either a sound wave with propagation angle $\chi$ (Chapter 1 ) or a gust (Chapters 2 and 3 ). The background steady flow shown here is uniform.

equation describing the distortion of the incident unsteady vorticity as it propagates downstream; and a second equation being the wave equation for the unsteady velocity potential, forced by the distorted vorticity. This method is used when we analyse both the gust- and sound-aerofoil interaction in uniform flow, and a similar decomposition of the flow field (into a gust part and a scattered acoustic part) occurs when we consider gust-aerofoil interaction in steady shear flow.

Single gust-aerofoil interaction is of fundamental importance for creating semi-analytic models for the sound generated by a gust interacting with an entire cascade of aerofoils. Peake \& Kerschen $(1997,2004)$ have considered the high-frequency far-upstream and -downstream acoustic radiation generated by a gust interacting with a cascade of flat plates at non-zero angles of attack; these solutions are constructed by taking the gustaerofoil interaction for a single blade, then assessing the rescattering of the acoustics generated by one blade with all of the other blades in the cascade. More complicated aerofoil geometries have been considered by Evers \& Peake (2002), however they only analysed the upstream acoustic radiation by considering just the leading-edge sources which are obtained by approximating a conformal mapping near the leading edges of each aerofoil, and applying the result from Peake \& Kerschen (1997). The downstream radiation is much more complicated (and a subject that Evers \& Peake (2002) do not consider), because each pair of blades in a cascade forms a duct down which acoustic modes can travel before rescattering at the trailing edge, or simply exiting the duct and propagating into the far field. The interaction of an incident sound wave with a blade row (sound-cascade interaction) has been examined by Amiet (1971) and Koch (1971) but only for flat-plate cascades at zero angle of attack. Amiet uses an asymptotic method to obtain a far-field high-frequency acoustic solution, whilst Koch develops a finite Wiener-Hopf problem which can be solved iteratively for the far-field acoustic pressure (unrestricted to a specific frequency regime). Neither of these works consider 
the single aerofoil sound-aerofoil interaction problem, nor do they consider aerofoils with realistic geometries. Even more recent sound-cascade studies such as Posson et al. (2013) still only consider flat plates at zero angle of attack, and in fact this mentioned work does not consider background steady flow interaction.

For (single) gust-aerofoil interaction in subsonic uniform flow, acoustic pressure is generated by the interaction of the unsteady vorticity with the solid aerofoil surface (momentum-blocking, which arises as a boundary condition) and by the distortion of the unsteady vorticity with the non-uniform flow around the aerofoil (which arises as a source term in the governing equations, dependent on the aerofoil geometry). The rapid distortion equations have been solved numerically for low- to mid-range frequencies for the gust-aerofoil problem, and we mention in particular the results of Atassi et al. (1993) and Scott \& Atassi (1995). Furthermore, computational aeroacoustic schemes (again at low- to mid-range frequencies) for the full non-linear Navier-Stokes or Euler equations have been developed (see Hixon et al. (2006) and Allampalli et al. (2009)). Turning to more analytically-based work, Myers \& Kerschen (1995, 1997), and Tsai (1992) have developed asymptotic solutions to the rapid distortion theory equations at high frequencies, accounting for aerofoil loading and camber, and thickness respectively. They used the equations for the modified velocity potential derived by Kerschen \& Balsa (1981) and Kerschen \& Myers (1987), and found the far-field acoustic pressure asymptotically in the limit of large gust reduced frequency $(k \gg 1)$ and small (but non-zero) aerofoil thickness and camber angle (denoted by $t=\epsilon t^{\prime}$ and $\alpha=\epsilon \alpha^{\prime}$ respectively, where $\epsilon \ll 1$ is an ordering parameter, in the preferred limit $\epsilon k=O(1))$. Their analysis is restricted to aerofoils with parabolic leading edges (nose radii scaling on thickness squared), so that the effects of the local stagnation point flow (such as the well known divergence of the drift in rapid distortion theory) occur in a region of size $O\left(\epsilon^{2}\right)$, and do not feature on the scale of the acoustic wavelength, $O(\epsilon)$.

Currently there are few comparisons available between analytic and numerical solutions due to the differing regimes in which each solution is determined and a lack of analytic solutions for realistic aerofoil geometries. Therefore validation of computational aeroacoustic (CAA) codes is of great concern. Various benchmark CAA solutions have been provided by Scott (2004), however these are only in the low- to mid-range frequency regime $(k \lesssim 3)$. Analytic solutions for leading-edge gust-aerofoil interaction noise have 
been obtained by Chapman (2003) for arbitrary gust disturbances ${ }^{1}$ interacting with flat plates, with the hope that higher frequency CAA codes will be able to use these for validation.

For sound-aerofoil interaction in subsonic uniform flow, which we discuss in detail in Chapter 1, acoustic pressure is generated both by scattering and by reflection and blocking of the incident sound wave by the solid aerofoil. The only mathematical difference between sound-aerofoil and gust-aerofoil interaction is in the upstream initial perturbation, hence the problem formulation from Goldstein (1978b), or more specifically, Myers \& Kerschen (1997) and Tsai (1992), can be adapted for use in this problem. We restrict our attention to aerofoils with parabolic leading edges since aerofoils with these nose shapes are typical of those used in industry in modern-day aircrafts, for example the NACA 4-digit series. We use the parameters for frequency, $k$, thickness, $t$, and camber angle, $\alpha$, as stated previously. Due to the ability to reflect and block sound in this system, there is a key difference in the far-field acoustic results from those obtained for gust-aerofoil interaction problems by Myers \& Kerschen (1997) and Tsai (1992); at the reflection and shadow angles, there is an acoustic field representing the total reflection and blocking of the incident sound wave by the whole of the aerofoil body (i.e. not specifically the leading or trailing edges). The corresponding field for gust-aerofoil interaction (that cancels the gust normal velocity along the whole body of the aerofoil) is hydrodynamic and does not propagate noise into the far field. Also at these shadow and reflection angles, we also see Fresnel regions which are generated by the interaction of the incident sound wave with the leading and trailing edges; these Fresnel regions form part of the scattered solution (which is distinctly different from the reflected and blocked solution) and are regions where the scattered acoustic pressure is greatest in magnitude. They are discussed in detail in Chapter 1. For distances far enough away from the aerofoil, the scattered Fresnel regions from the leading and trailing edges overlap and dominate the reflection/blocking solution, thus the Fresnel solutions are all that are seen along the reflection and shadow boundaries. A discussion of this overlap of scattered Fresnel fields and direct reflection/blocking fields can be found in Peake \& Kerschen (2004), where the noise emanating from the rear of a cascade is considered; acoustic duct modes can scatter by interaction with the trailing edge (creating Fresnel regions) but also be emitted directly into the far field without any trailing-edge interaction.

\footnotetext{
${ }^{1}$ For work in this dissertation we will be using single-frequency sinusoidal gusts whose far-upstream profiles are $\boldsymbol{A} \mathrm{e}^{\mathrm{i} \boldsymbol{k} \cdot \boldsymbol{x}-\mathrm{i} \omega t}$, however the "arbitrary" gusts in Chapman (2003) refer to those comprising of a longitudinal shape function, $f_{0}$, and a transverse shape function, $g_{0}$, so that the gust may be thought of as having vertical velocity component $f(t-x / U) g(z)$ on $y=0$. The wider range of gusts considered allows for more rigorous CAA testing as more variable parameters are permitted.
} 
In Chapter 2 we take advantage of the analysis completed in Chapter 1, and the previous work by Myers \& Kerschen (1997) and Tsai (1992), to construct an analytic solution for the uniform flow gust-aerofoil interaction problem for aerofoils with small thickness, camber, and angle of attack, which until now has not been done. We compare our generalised result to results from Myers \& Kerschen (1997) and Tsai (1992) as well as current mid-frequency CAA solutions.

Acoustic pressure is generated by a different mechanism for gust-aerofoil interaction in steady subsonic shear flow. In uniform flow, a gust is purely convective and creates no acoustic pressure perturbations itself, so the sound generated comes only from the interaction of the gust with the body. In shear, however, the gust distorts in the background flow causing acoustic perturbations to arise; this is known as gust self-noise. When the gust interacts with the aerofoil in shear, sound is generated by the blocking of non-acoustic gust velocity components (the source of sound in uniform flow), and by the scattering and reflection of the gust self-noise. This new process means that separating the unsteady flow solution into vortical and irrotational parts is no longer sensible. Instead, the unsteady solution is separated into a "gust solution" that describes the evolution of the gust as it distorts through the steady background shear, and an outward-propagating acoustic part, that describes the acoustics generated by the gust interacting with the aerofoil. The gust solution has been considered previously by Goldstein $(1978 a, 1979)$, and the sound generated by a gust interacting with a semi-infinite flat plate has been found. We greatly extend this work in Chapter 3 to investigate the sound generated by a gust interacting with a finite symmetric aerofoil with small but non-zero thickness.

It shall be found that during gust-aerofoil interaction in uniform steady flow, a singularity occurs in the unsteady pressure at the leading edge. This is clearly a violation of the small perturbation assumption used during the asymptotic analysis ${ }^{2}$. Chapter 4 therefore addresses this singularity by considering the leading edge more closely than in the other chapters. We discuss gusts of arbitrary (i.e. not specifically high) frequency incident on an ellipse with small but non-zero thickness. Since any gust frequency is allowable, we then investigate the effects of upstream homogeneous isotropic turbulence since turbulence of this variety can be decomposed as a Fourier series of gusts (Hunt, 1973). We compare asymptotic results to experimental findings for the one-dimensional turbulent pressure spectrum at the leading edge of bodies with small but non-zero thickness.

\footnotetext{
${ }^{2}$ This violation occurs only on the aerofoil surface and does not affect the far-field results except potentially at a single point directly upstream of the aerofoil (at $\theta=\pi$ on a standard polar plot).
} 
The layout of this dissertation is as follows; we begin by discussing the new topic of sound-aerofoil interaction in subsonic steady flow in Chapter 1, presenting all relevant calculations. This chapter has been published as Ayton \& Peake (2013). In Chapter 2 we review the procedures of Myers \& Kerschen (1997) and Tsai (1992), extending them and using results from Chapter 1 to consider gust-aerofoil interaction in subsonic steady flow for aerofoils of small thickness, camber, and angle of attack. We present only key results in the main body, with most calculations given in the appendices where necessary, since much of the method is similar to that in Chapter 1. In Chapter 3 we discuss gust-aerofoil interaction in subsonic shear flow, greatly extending the work from Goldstein (1978a). This chapter is outlined in Ayton \& Peake (2014a), and has been submitted for publication as Ayton \& Peake (2014b). Chapter 4 addresses the problem of the singularity in the unsteady pressure on the nose of an aerofoil arising during gustaerofoil interaction in uniform flow. Conclusions and suggestions for further work are presented in Chapter 5

In each chapter, relevant functions and variables are defined independently of all other chapters, unless otherwise stated. Each chapter contains all results and conclusions relevant to the work in the chapter, and is followed by relevant appendices. A list of symbols for each chapter can be found immediately after the main body of work in each chapter, prior to any appendices. References feature at the end of the dissertation. 


\section{Chapter 1}

\section{High-Frequency Sound Generated by Sound-Aerofoil Interaction in Subsonic Uniform Flow}

In this chapter we adapt the analytically-based procedure of Myers \& Kerschen (1995, 1997), and Tsai (1992) to study the sound-aerofoil interaction problem. We consider a high-frequency incident plane sound wave being scattered by a generic aerofoil (such as that in Figure 0.7) in a steady uniform background flow, and use the asymptotic limits as described in the Introduction; $k \gg 1$ is the acoustic reduced frequency; $\epsilon \ll 1$ is a non-dimensionalised small parameter (lengths are non-dimensionalised with respect to semi-chord length) such that $t=\epsilon t^{\prime}$ and $\alpha=\epsilon \alpha^{\prime}$ are the thickness and camber, respectively, of the aerofoil; and we impose that $\epsilon k=O(1)$. Results will be shown specifically for NACA 4-digit series aerofoils, however any aerofoil with a parabolic nose is allowable. In Section 1.2 we discuss this nose shape constraint and allude to results for bodies with different nose shapes, and later in Chapter 4 we explicitly discuss ellipticnosed bodies (although in both of these cases the radius of curvature of the nose is $O\left(\epsilon^{2}\right)$ ). The method of matched asymptotic expansions, (Van Dyke, 1975), is used to split the problem into several asymptotic regions around the aerofoil; the inner leading- and trailing-edge regions, of size $O\left(k^{-1}\right)$ centred on the leading and trailing edges respectively; the transition regions on the aerofoil surfaces between the inner leading- and trailing-edge regions, of width $O\left(k^{-1 / 2}\right)$, which account for the surface curvature of the aerofoil; a wake region, also of width $O\left(k^{-1 / 2}\right)$; and the outer region comprising the rest of space, which also contains Fresnel zones above and below the aerofoil emanating from the leading and trailing edges. The solution in each region is determined individually, and matched to its surrounding regions using Van Dyke's matching rule (Van Dyke, 1975). An advantage of this analytical approach is that it provides results in the high-frequency regime (where numerical approaches become more difficult) as well as providing interesting physical insight.

In Section 1.1 we formulate the mathematical problem for the incidence of a highfrequency sound wave on an aerofoil. In Section 1.2 we solve the governing equations 
in the inner leading-edge region of the aerofoil. On taking the outer limit of this inner solution (as required by the matching process) we find singularities along the shadow and reflection boundaries, so we construct uniformly-valid expansions to eliminate these singularities in the far-field solution. The leading-order solution (the flat-plate solution) yields the well-known uniformly-valid expansion containing the complementary error function, and is caused by the coalescence of a saddle point and a pole in a very familiar way (Jones, 1986). In contrast, the part of the leading-order thickness correction which accounts for the inclination of the surface normal vector has a singularity caused by the coalescence of a branch cut and a saddle point, and the uniformly-valid solution is more unusual, containing a modified Bessel function of the second kind. In Section 1.3 we construct the leading-edge outer, and surface transition solutions, the latter of which accounts for the surface curvature of the aerofoil. In Section 1.4 we find the trailing-edge outer, transition and inner solutions; the inner solution comprises of the field arising from the scattering of the leading-edge field by the trailing edge, which is similar to that found in Myers \& Kerschen (1997) and Tsai (1992), and the reflected field, which is particular to our incident sound problem. This reflected field generates further Fresnel regions, now emanating from the trailing edge, which have the standard far-field form involving the complementary error function. The final uniformly-valid result of the total far-field sound generated when a sound wave is scattered and reflected by a thin aerofoil in background steady uniform flow is presented in Section 1.5. Section 1.6 contains results and discussion, and concluding remarks are given in Section 1.7.

\subsection{Formulation of the Problem}

\subsubsection{Aerofoil Geometry and Steady Mean Flow}

We consider a small unsteady perturbation to the mean flow around a thin aerofoil of chord length $2 b^{*}$ in Cartesian coordinates $\left(x^{*}, y^{*}\right)$ with their origin at the aerofoil leading edge. We use ${ }^{*}$ to denote dimensional quantities. We restrict the geometry of the aerofoil to have a parabolic nose $\left(\epsilon y^{*}\left(x^{*}\right) \sim 2 a \epsilon t^{\prime} \sqrt{x^{*} / b^{*}}\right.$ at the leading edge, where $t=\epsilon t^{\prime}$ is the maximum thickness of the aerofoil as a fraction of the chord length and primed quantities are $O(1))$ and a sharp trailing edge. We decompose the boundary description of the aerofoil, $\epsilon y^{*}\left(x^{*}\right)$, into thickness related terms, $\epsilon y^{(t) *}$, and camber and angle of attack related terms, $\epsilon Y^{(c) *}\left(x^{*}\right)=-\alpha_{i} x^{*}+\epsilon y^{(c) *}\left(x^{*}\right)$, where $\alpha_{i}$ is the angle of attack, and $y^{(c)}$ describes the camber line of the aerofoil.

In what follows we non-dimensionalise lengths with respect to $b^{*}$, and velocities by 
$U_{\infty}^{*}$, where $U_{\infty}^{*}$ is the uniform mean flow speed far from the aerofoil. Note this nondimensionalisation fails when there is no background mean flow, but we do not consider such a simplified case. At upstream infinity the mean flow is uniform, with speed $U_{\infty}^{*}$ aligned with the positive $x^{*}$ direction. It is most convenient to work in the nondimensionalised orthogonal coordinate system $(\phi, \psi)$, which represent the velocity potential and streamfunction of the mean flow around the aerofoil. In this coordinate system we write $z=\phi+\mathrm{i} \psi$, which relates to the Cartesian coordinates $(x, y)$ via

$$
x+\mathrm{i} \beta_{\infty} y=z+O(\epsilon)
$$

where $\beta_{\infty}^{2}=\left(1-M_{\infty}^{2}\right)$ is the Prandtl-Glauert transformation factor accounting for compressibility, and $M_{\infty}$ is the mean flow Mach number at upstream infinity.

To utilise the modified rapid distortion theory equations determined by Kerschen \& Myers (1987), we need the complex potential for the steady flow around the aerofoil. Using Thwaites (1960) we find that, for a thin aerofoil, the amplitude of the total mean flow is $U_{\infty}^{*}(1+\epsilon q)$, in the direction making an angle $\epsilon \mu$ with the $x^{*}$ axis, where

$$
(q-\mathrm{i} \mu)(z)=\frac{1}{\pi \beta_{\infty}} \int_{0}^{2} \frac{y^{(t) \prime}(x)}{z-x} d x+\frac{1}{\pi \beta_{\infty}} \sqrt{\frac{2-z}{z}} \int_{0}^{2} \frac{Y^{(c) \prime}(x)}{z-x} \sqrt{\frac{x}{2-x}} d x
$$

and ' denotes differentiation. This is correct to first order in aerofoil thickness, camber and angle of attack. The quantities $q$ and $\mu$ are related to the complex potential $F$ (which is non-dimensionalised with respect to $U_{\infty}^{* 2} b^{*}$ ) by

$$
q(\phi, \psi)-\mathrm{i} \mu(\phi, \psi)=\frac{d F}{d z}
$$

where the arbitrary constant in $F$ is chosen so that $F(0)=0$. This now completes the relation given by (1.1.1.1); the $O(\epsilon)$ perturbation is $\epsilon F(z)$. We require explicit details of $q$ and $\mu$ in the local neighbourhood of the leading and trailing edges. For the other regions we construct the unsteady solution in terms of $q, \mu$, and $F$. For further details of the coordinate transformation between physical $(x, y)$-space, and potential-streamline space, see Myers (1987).

We combine the angle of attack, $\alpha_{i}=\epsilon \alpha_{i}^{\prime}$, and the leading-edge camber together to give an effective angle of attack, $\alpha_{\text {eff }}=\epsilon \alpha_{\text {eff }}^{\prime}$, with

$$
\alpha_{\mathrm{eff}}=\alpha_{i}-\frac{1}{\pi} \int_{0}^{2} \frac{\epsilon y^{(c)}(x)(1-x)}{((2-x) x)^{3 / 2}} d x
$$


Equation (1.1.1.4) has been found by sending $z \rightarrow 0$ in the second term in (1.1.1.2) and integrating by parts so that later, with this effective angle of attack, we may write the inner leading-edge expansion of $q$ as $q-\mathrm{i} \mu \sim \alpha_{\text {eff }}^{\prime} \beta_{\infty}^{-1} \sqrt{2} z^{-1 / 2}+$ thickness term + $O\left(z^{1 / 2}\right)$. The complex velocity potential for a general thin aerofoil with boundary $\epsilon y(x)=$ $\epsilon y^{(t)}(x)+\epsilon y^{(c)}(x)$, where $t$ and $c$ denote thickness and camber respectively, can be written as $F=F_{0}+F^{(t)}+F^{(c)}$, where $F_{0}$ is the flat-plate potential at angle of attack $\alpha_{i}$ as given in Myers \& Kerschen (1995). The flat-plate (at the angle of attack), thickness and camber dependent components of the complex potential are given by

$$
\begin{aligned}
F_{0}(z)= & \frac{\mathrm{i} \alpha_{i}}{\beta_{\infty}}(\log [z-1+\sqrt{z(z-2)}]+z-\sqrt{z(z-2)}-\pi \mathrm{i}), \\
F^{(c)}(z)= & \frac{-\mathrm{i}}{\pi \beta_{\infty}} \int_{0}^{2} y^{(c) \prime}(x) \frac{\sqrt{x}}{\sqrt{2-x}}\left(\log \left[\frac{1+\sqrt{(z-2) / z}}{1-\sqrt{(z-2) / z}}\right]\right. \\
& \left.-\mathrm{i} \frac{\sqrt{2-x}}{\sqrt{x}} \log \left[\frac{\mathrm{i} \sqrt{(2-x) / x}+\sqrt{(z-2) / z}}{\mathrm{i} \sqrt{(2-x) / x}-\sqrt{(z-2) / z}}\right]-\pi \mathrm{i}\right) d x, \\
F^{(t)}(z)= & \frac{1}{\pi \beta_{\infty}}\left(\int_{0}^{2} y^{(t) \prime}(x) \log [z-x] d x+\int_{0}^{2} \frac{y^{(t) \prime}(x)}{x} d x\right) .
\end{aligned}
$$

This completes our description of the mean flow.

A shift of $z \rightarrow z+2$ recentres the expressions for the disturbance potentials about the trailing edge (where now we take constants such that the velocity potential with respect to trailing-edge coordinates, $F_{t}$, satisfies $\left.F_{t}\left(z_{t}=0\right)=0\right)$. These are used later to determine the phase shift between the leading- and trailing-edge scattered fields.

\subsubsection{Unsteady Disturbance Equations}

Rapid distortion theory is often used to investigate the sound generated by the interaction of an unsteady flow with a solid boundary. The theory was initially developed by Hunt (1973) and Goldstein (1978b) for three-dimensional incompressible and compressible flows respectively, but the form given by Kerschen \& Myers (1987) and Kerschen \& Balsa (1981) for two-dimensional, small-disturbance mean flows is more relevant to this chapter. We assume that the fluid is inviscid and non heat conducting, which is a reasonable assumption in many aeronautical applications such as fan noise. For the purposes here we assume that the incident sound wave originates far from the aerofoil where the mean flow is uniform, with a small-amplitude unsteady disturbance. The third coordinate in our orthogonal system $\left(\phi^{*}, \psi^{*}, x_{3}^{*}\right)$ is the spanwise direction, and we assume that all unsteady quantities are proportional to $\mathrm{e}^{\mathrm{i} k_{3} x_{3}-i \omega t}$, where $\omega$ is the non-dimensionalised 
frequency. We non-dimensionalise coordinates $\left(\phi^{*}, \psi^{*}\right)$ with respect to $U_{\infty}^{*} b^{*}$, wavevector $\boldsymbol{k}=k^{*}\left(k_{1}, k_{2}, k_{3}\right)$ with respect to $\left(b^{*} U_{\infty}^{*}\right)^{-1}$ and frequency with respect to $k^{*} U_{\infty}^{* 2}$.

We assume the perturbation to the uniform flow, due to the presence of the aerofoil, is small compared to the uniform flow component, but large compared to the unsteady flow component. Following Kerschen \& Myers (1987) we use the perfect gas relations for a small velocity perturbation, $\epsilon q$, to a uniform mean flow and take directly from them the governing equation for the modified unsteady velocity potential $h(\phi, \psi)$ (Kerschen \& Myers, 1987, eq. 10). Here $h$ relates to the physical unsteady velocity potential, $G$, via

$$
h(\phi, \psi)=G(\phi, \psi) \mathrm{e}^{-\mathrm{i} k_{3} x_{3}+\mathrm{i} \omega t} \mathrm{e}^{\mathrm{i} k M_{\infty}^{2} \phi / \beta_{\infty}-M_{\infty}^{2} \epsilon q},
$$

and $G$ satisfies

$$
\frac{D_{0}}{D t}\left(\frac{1}{a_{0}} \frac{D_{0} G}{D t}\right)-\frac{1}{\rho_{0}} \boldsymbol{\nabla} \cdot\left(\rho_{0} \boldsymbol{\nabla} G\right)=\frac{1}{\rho_{0}} \boldsymbol{\nabla} \cdot\left(\rho_{0} \boldsymbol{v}\right),
$$

where the perturbation to the steady mean flow field is given by $\boldsymbol{u}=\boldsymbol{v}+\boldsymbol{\nabla} G$ and $\boldsymbol{v}$ is the evolution of the incident vortical disturbance in the steady mean flow. In Goldstein (1978b), the flow field is separated into $\boldsymbol{v}$ and $G$ because (in the case of an incident gust) one, $G$, can then be seen as an acoustic field, and the other, $\boldsymbol{v}$, a vortical field. In this chapter, we are considering the effects of an acoustic incident field only, therefore we set $\boldsymbol{v}=\mathbf{0}$, and we separate $G$ into two parts, one that is the imposed perturbation, and one that is the response. Individually both fields satisfy (1.1.2.2) with zero source term. We solve (1.1.2.2) for the imposed incident field in the next subsection, whilst the remainder of the chapter focuses on solving for the response by imposing a boundary condition on the aerofoil surface. The incident sound field is denoted by a subscript ${ }_{I}$ whilst the response has no subscript.

The subscript ${ }_{0}$ denotes steady mean flow quantities, and $\rho_{0}$ and $a_{0}$ are density and speed of sound respectively. We can determine the mean flow quantities with respect to their values far upstream (denoted by subscript $\infty$ ), via

$$
\begin{aligned}
\frac{U_{0}}{U_{\infty}}=1+\epsilon q, & \frac{V_{0}}{V_{\infty}}=\epsilon \mu \\
\frac{a_{0}}{a_{\infty}}=1-\epsilon \frac{\gamma-1}{2} M_{\infty}^{2} q, & \frac{\rho_{0}}{\rho_{\infty}}=1-\epsilon M_{\infty}^{2} q, \\
\frac{\beta_{0}}{\beta_{\infty}}=1-\epsilon\left(1+\frac{\gamma-1}{2} M_{\infty}^{2}\right) \frac{M_{\infty}^{2}}{\beta_{\infty}^{2}} q, & \frac{M_{0}}{M_{\infty}}=1+\epsilon\left(1+\frac{\gamma-1}{2} M_{\infty}^{2}\right) q .
\end{aligned}
$$

These expansions are correct to first order in $\epsilon$, and $\gamma$ is the ratio of specific heats.

After non-dimensionalisation of (1.1.2.2), we retain terms of size $O\left(k^{2}, k, 1\right)$ (recall 
$\left.k=O\left(\epsilon^{-1}\right)\right)$, to find the governing equation for $h$,

$$
\begin{array}{r}
\frac{\partial^{2} h}{\partial \phi^{2}}+\frac{\partial^{2} h}{\partial \psi^{2}}+k^{2} w^{2}\left(1-2 \beta_{\infty}^{2} \epsilon q\right) h+\frac{(\gamma+1) M_{\infty}^{4} \epsilon q}{\beta_{\infty}^{2}}\left(\frac{\partial^{2} h}{\partial \psi^{2}}+2 \mathrm{i} k \delta \frac{\partial h}{\partial \phi}+k^{2}\left(w^{2}+\delta^{2}\right) h\right) \\
-\frac{(\gamma+1) M_{\infty}^{4}}{\beta_{\infty}^{2}} \epsilon \frac{\partial q}{\partial \phi}\left(\frac{\partial h}{\partial \phi}-\mathrm{i} k \delta h\right)=0, \quad(1.1 .2 .4 a)
\end{array}
$$

where

$$
\delta=\frac{\omega}{\beta_{\infty}^{2}}, \quad w^{2}=\left(\delta M_{\infty}\right)^{2}-\left(k_{3} / \beta_{\infty}\right)^{2}
$$

In (1.1.2.4) we have now explicitly set the source term from (1.1.2.2) to zero.

We work with the modified unsteady pressure, as defined by Myers \& Kerschen (1995, eq. (2.7)), which is given by

$$
p=-\left(\frac{\partial h}{\partial \phi}-\mathrm{i} k \delta h\right) \mathrm{e}^{-\mathrm{i} k \delta M_{\infty}^{2} \phi}
$$

In the following sections we solve for $h$ in various regions around the aerofoil, wherein different terms dominate the behaviour of the flow. These regions arise from balancing different terms in (1.1.2.4) with respect to $k$ and $\epsilon$. We have a particular aim of finding the acoustic pressure in the far field.

\subsubsection{Form of the Incident Sound}

Suppose the incident sound wave has potential

$$
h_{I}=A_{I} \mathrm{e}^{\mathrm{i} k w \sigma_{I}}
$$

We require $A_{I}=1$, and $\sigma_{I}=k_{1} \phi+k_{2} \psi$ at infinity, where $k_{1,2}$ are the streamwise and transverse wavenumbers of the incident sound wave. Writing $\sigma_{I}=\sigma_{0}+\epsilon \sigma_{1}+O\left(\epsilon^{2}\right)$ and substituting (1.1.3.1) into (1.1.2.4) we find that to $O\left(k^{2}\right)$

$$
\left(\frac{\partial \sigma_{0}}{\partial \phi}\right)^{2}+\left(\frac{\partial \sigma_{0}}{\partial \psi}\right)^{2}-1=0
$$

Hence $\sigma_{0}=k_{1} \phi+k_{2} \psi$ subject to $k_{1}^{2}+k_{2}^{2}=1$. We define $\chi$ as the angle of propagation of the sound wave with respect to the uniform flow upstream, and write $\left(k_{1}, k_{2}\right)=$ $(\cos \chi, \sin \chi)$. The sound originates from upstream, $-\pi / 2<\chi<\pi / 2$, or from down- 
stream $\pi / 2<\chi<3 \pi / 2$. The real part of (1.1.2.4) to $O(k)$ gives the eikonal equation

$$
\cos \chi \frac{\partial \sigma_{1}}{\partial \phi}+\sin \chi \frac{\partial \sigma_{1}}{\partial \psi}+q\left(\beta_{\infty}^{2}-\frac{(\gamma+1) M_{\infty}^{4}}{2 \beta_{\infty}^{2} w^{2}}(\delta-w \cos \chi)^{2}\right)=0
$$

whilst the imaginary part at this order gives the transport equation

$$
\frac{\partial A_{I}}{\partial \phi} \cos \chi+\frac{\partial A_{I}}{\partial \psi} \sin \chi=0
$$

By using characteristic coordinates $\xi=\phi+\psi \cot \chi, \eta=\phi-\psi \cot \chi$ and re-labelling $q(\phi, \psi)$ as $\mathscr{Q}(\xi, \eta)$ in these variables, we obtain the solution to the eikonal equation

$$
\sigma_{1}=\frac{V(\chi)}{2 \cos \chi} \int_{-\infty}^{\xi} \mathscr{Q}\left(\xi^{\prime}, \eta\right) d \xi^{\prime}
$$

where

$$
V(\theta)=-\beta_{\infty}^{2}\left(1-\frac{(\gamma+1) M_{\infty}^{4}}{2 \beta_{\infty}^{4} w^{2}}(\delta-w \cos \theta)^{2}\right) .
$$

The function $\sigma_{1}$ represents the distortion of the incident sound wave due to the nonuniform steady flow created by the presence of the aerofoil. We set $g_{1}$ to zero due to the condition at infinity. The transport equation is satisfied by an arbitrary function of the spanwise coordinate, $x_{3}$, but given the condition at infinity we set $A_{I}=1$ everywhere, and hence the incident sound wave is given by

$$
h_{I}=\exp \left(\mathrm{i} k w(\phi \cos \chi+\psi \sin \chi)+\frac{\mathrm{i} k \epsilon w V(\chi)}{2 \cos \chi} \int_{-\infty}^{\xi} \mathscr{Q}\left(\xi^{\prime}, \eta\right) d \xi^{\prime}\right)
$$

If $\chi=\pi / 2$, we can solve for $\sigma_{1}$ directly from (1.1.3.3) without changing to characteristic variables. This special case shall not be considered further. The boundary condition for the problem is one of zero unsteady normal velocity on the aerofoil surface, i.e.

$$
\left(\frac{\partial h}{\partial \psi}+M_{\infty}^{2} \epsilon \frac{\partial q}{\partial \psi} h\right)_{\psi=0}=-\left(\frac{\partial h_{I}}{\partial \psi}+M_{\infty}^{2} \epsilon \frac{\partial q}{\partial \psi} h_{I}\right)_{\psi=0}
$$

Here $\psi=0$ corresponds to the aerofoil surface and the range of $\phi$ over which (1.1.3.8) holds corresponds to the body length in the $(\phi, \psi)$ plane.

We use the method of matched asymptotic expansions (Van Dyke, 1975) to solve the problem in all regions shown in Figure 1.1; solving (i) and (ii) in Section 1.2, specifically with (ii) occurring in Sections 1.2.1, 1.2.2, 1.2.4 and 1.2.5; solving (iii) in Sections 1.3.1 and 1.4.1; solving (iv) in Section 1.3.2; solving (v) and (vi) in Section 1.4.2; and finally 


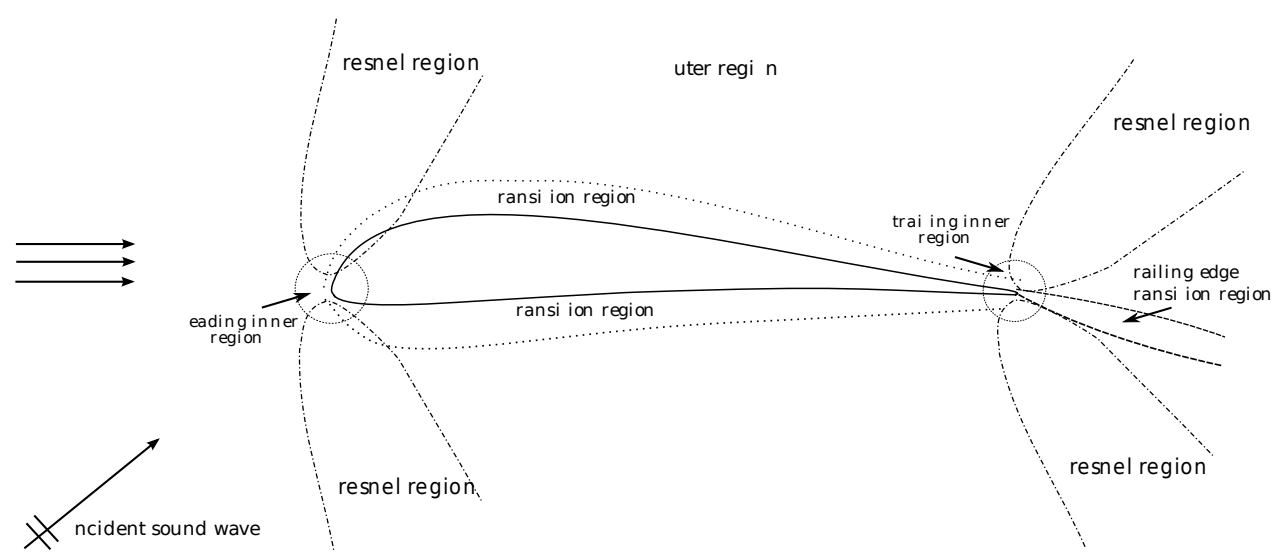

(a) In $(x, y)$ space.

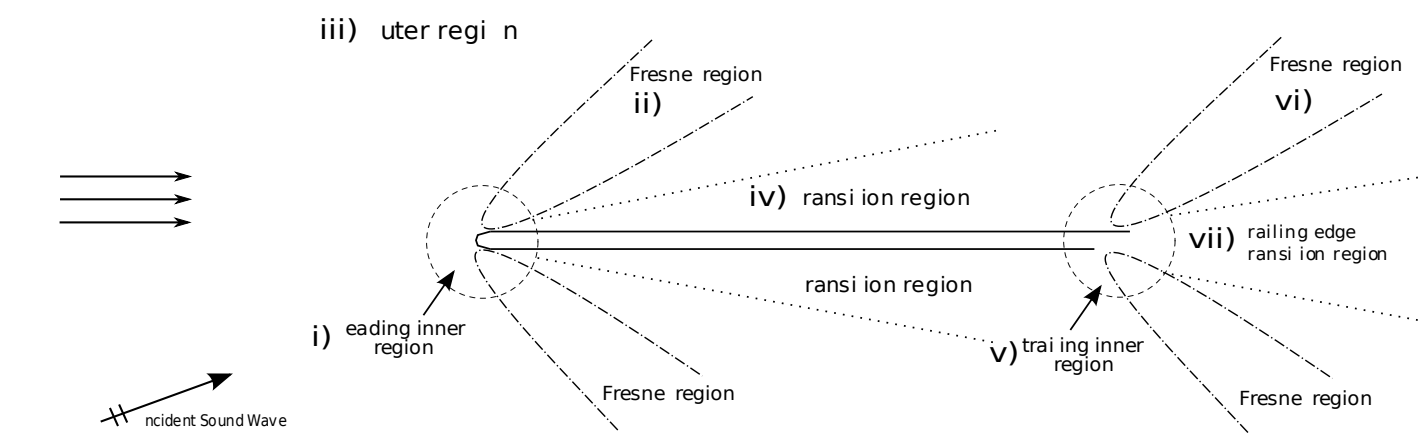

(b) In $(\phi, \psi)$ space.

Figure 1.1: Asymptotic regions around the aerofoil; leading- and trailing-edge inner regions, (i) and (v), scale as $O\left(k^{-1}\right)$, the width of the transition regions, (iv) and (vii), scales as $O\left(k^{-1 / 2}\right)$, and the width of the Fresnel regions, (ii) and (vi), scales as $O\left((k r)^{1 / 2}\right)$. The outer region (iii) is $O(1)$ on the scale of the aerofoil chord. We solve for (i) in Section 1.2, with (ii) found specifically in Sections 1.2.1, 1.2.2, 1.2.4, and 1.2.5. We then solve for a leading-edge contribution to (iii) in Section 1.3.1, and (iv) in Section 1.3.2. We solve for the trailing-edge contribution to (iii) in Section 1.4.1, solve for (v) and (vi) in Section 1.4.2, and finally for (vii) in Section 1.4.3 
solving (vii) in Section 1.4.3. As in Myers \& Kerschen (1997), we take $k \gg 1$ and $\epsilon \ll 1$ with the distinguished limit that $\epsilon k=O(1)$, but with suitable choice of $O(1)$ parameters $t^{\prime}$ and $\alpha^{\prime}$ (recall thickness and camber angle of the aerofoil are measured by, $t=\epsilon t^{\prime}$ and $\alpha=\epsilon \alpha^{\prime}$ ), our analysis also holds for flat-plate or uncambered aerofoils. We begin with the leading-edge inner region, then match that to the leading-edge outer region. The leading-edge transition region along the aerofoil surface can then be constructed. A similar process is completed at the trailing edge, but to ensure continuity of pressure at the trailing edge, these solutions rely on the leading-edge solution, and thus are more complicated.

\subsection{Leading-Edge Inner Solution}

Here we introduce inner variables $(\Phi, \Psi)=k(\phi, \psi)$ for $k \gg 1$ centred on the leading edge, and require the inner expansion of (1.1.1.2). To obtain this, we take the approximation of the aerofoil boundary near the leading edge in inner variables, substitute into (1.1.1.2), and expand for large $k$. Recalling that $y^{(t)} \sim 2 a \epsilon t^{\prime} \sqrt{x}$ as $x \rightarrow 0$, and $\alpha_{\text {eff }}$ is defined in (1.1.1.4) as a function of the angle of attack and the camber distribution, the result is

$$
(q-\mathrm{i} \mu)(R, \theta) \sim-\frac{a t^{\prime} \mathrm{i}}{\beta_{\infty}} \sqrt{\frac{k}{R}}(\cos \theta / 2-\mathrm{i} \sin \theta / 2)+\frac{\alpha_{\text {eff }}^{\prime}}{\beta_{\infty}} \sqrt{\frac{2 k}{R}}(\cos \theta / 2-\mathrm{i} \sin \theta / 2),
$$

where $(R, \theta)$ are the local polar coordinates centred on $(0,0)$ with $R=k r$. Transforming the equations (1.1.2.4) gives

$$
\begin{array}{r}
\frac{\partial^{2} H}{\partial \Phi^{2}}+\frac{\partial^{2} H}{\partial \Psi^{2}}+w^{2}\left(1-2 \beta_{\infty}^{2} \epsilon q\right) H+\frac{(\gamma+1) M_{\infty}^{4}}{\beta_{\infty}^{2}} \epsilon q\left(\frac{\partial^{2} H}{\partial \Psi^{2}}+2 \mathrm{i} \delta \frac{\partial H}{\partial \Phi}+\left(w^{2}+\delta^{2}\right) H\right) \\
-\frac{(\gamma+1) M_{\infty}^{4}}{\beta_{\infty}^{2}} \epsilon \frac{\partial q}{\partial \Phi}\left(\frac{\partial H}{\partial \Phi}-\mathrm{i} \delta H\right)=0, \quad(1.2 .0 .2
\end{array}
$$

subject to the boundary condition of zero normal velocity on the aerofoil surfaces, $\psi=0$, i.e.

$$
\frac{\partial H}{\partial \Psi}+\left.M_{\infty}^{2} \epsilon \frac{\partial q}{\partial \Psi} H\right|_{\Psi=0 \pm}=-\left[\frac{1}{k} \frac{\partial h_{I}}{\partial \psi}+M_{\infty}^{2} \epsilon \frac{\partial q}{\partial \Psi} h_{I}\right]_{\Psi=0 \pm}
$$

where $H$ is the unsteady potential in the inner region. This boundary condition tells us that $H=O(1)$ at leading order. We now follow Myers \& Kerschen (1997) and Tsai 
(1992), and expand the inner unsteady potential in the form;

$$
H(\Phi, \Psi)=\mathrm{e}^{\mathrm{i} k w \sigma_{1}(0,0)}\left(H_{0}+\epsilon t^{\prime} \sqrt{k}\left(H_{1}+H_{2}+H_{3}\right)+\epsilon \alpha_{\text {eff }}^{\prime} \sqrt{k}\left(P_{1}+P_{2}+P_{3}\right)+O(\epsilon)\right)
$$

Here, the phase function, $\sigma_{1}$, is given by (1.1.3.5) and arises due to the distortion of the incident sound wave through the non-uniform flow at the nose. Of the remaining functions, $H_{0}$ is the flat plate solution at zero angle of attack, $H_{1,2,3}$ are thicknessdependent terms, and $P_{1,2,3}$ are camber and angle of attack dependent terms. In what follows we determine each of these terms.

We mention here the requirement to have a parabolic-nosed body, $y \sim 2 a \epsilon t^{\prime} \sqrt{x}$. We choose this shape so that we may analyse the inner region qualitatively, and in particular obtain (1.2.0.1) and (1.2.0.3). For an arbitrary nose, $y \sim \epsilon t^{\prime} x^{m}$, where $0<m<1$ we would obtain $q \sim(k / R)^{m-1}$ thus the asymptotic series, (1.2.0.3), would be $H_{0}+\epsilon k^{m} \mathcal{H}_{1}$. We would proceed to match orders of $\epsilon k^{m}$ in the governing equation and boundary conditions as illustrated in the coming sections, but would have an additional arbitrary quantity, $m$. The parabolic nose choice, $m=1 / 2$, conforms to the NACA 4-digit series of aerofoil that are commonplace in the literature of blade-blade interaction problems, hence we impose this from the beginning to ease the understanding of the analysis.

\subsubsection{Solution for the Flat-Plate Term $H_{0}$}

The leading-order solution is simply that found for a semi-infinite flat plate at zero angle of attack with an incident sound wave in uniform flow. It satisfies

$$
\begin{aligned}
D\left(H_{0}\right) & =0, \\
\left.\frac{\partial H_{0}}{\partial \Psi}\right|_{\substack{\Phi>0 \\
\Psi=0}} & =-\mathrm{i} w \sin \chi \mathrm{e}^{\mathrm{i} w \cos (\chi) \Phi},
\end{aligned}
$$

where

$$
D=\frac{\partial^{2}}{\partial \Phi^{2}}+\frac{\partial^{2}}{\partial \Psi^{2}}+w^{2}
$$

This is of the same form as the flat-plate problem for an incident gust, and only depends on the aerofoil surface blocking the normal component of the incident velocity. Using the Wiener-Hopf technique in a standard way (see Myers \& Kerschen (1995) for more details), we obtain the solution

$$
H_{0}=-\frac{\mathrm{i} \sqrt{w} \sin (\chi) \operatorname{sgn}(\Psi)}{2 \pi \sqrt{\cos \chi+1}} \int_{-\infty}^{\infty} \frac{\mathrm{e}^{-\mathrm{i} \lambda \Phi-|\Psi| \sqrt{\lambda^{2}-w^{2}}}}{(\lambda+w \cos \chi) \sqrt{\lambda+w}} d \lambda
$$


Expanding (1.2.1.3) for large $R$, for later matching to the outer solution, gives

$$
\begin{aligned}
H_{0} & \sim L_{0}(\theta) \frac{\mathrm{e}^{\mathrm{i} k w r}}{\sqrt{k r}}+H_{0}^{p}+O\left(k^{-3 / 2}\right), \\
L_{0}(\theta) & =-\frac{\mathrm{i} \sin \chi \mathrm{e}^{-\mathrm{i} \pi / 4} \cos \theta / 2}{\sqrt{\pi} \sqrt{w \cos \chi+w}(\cos \chi-\cos \theta)} .
\end{aligned}
$$

$H_{0}^{p}$ is the contribution from the pole at $\lambda=-w \cos \chi$, which may or may not be included, depending on where the pole lies in relation to the steepest descent contour for the function

$$
a(\lambda)=-\mathrm{i} \lambda R \cos \theta-R|\sin \theta| \sqrt{\lambda^{2}-w^{2}} .
$$

See Figure 1.2 for an illustration of the steepest descent contour. In fact,

$$
H_{0}^{p}=-\operatorname{sgn}(\Psi) \mathrm{e}^{\mathrm{i} w \cos (\chi) \Phi+\mathrm{i} w \sin (\chi)|\Psi|}
$$

if $0<\theta<\chi$ or $2 \pi-\chi<\theta<2 \pi$, and $H_{0}^{p}$ is zero otherwise. This pole represents shadow, $0<\theta<\chi$, and reflection, $2 \pi-\chi<\theta<2 \pi$, regions of the incident sound wave by a semi-infinite flat plate. There is no pole in the gust case of Myers \& Kerschen (1997), therefore for them, $L_{0}$ would be uniformly valid, however for the sound-aerofoil case, our expression for $L_{0}$ has two regions of non-uniformity, i.e. when $\cos \chi=\cos \theta$, which is caused in a very familiar way by the coincidence of the pole in the integrand of (1.2.1.3) and the saddle point arising in the determination of the outer limit.

The uniformly-valid outer limit of (1.2.1.3) can be found using standard methods (Jones, 1986) which we outline here; upon deforming the contour to the steepest descents contour (see Figure 1.2) we cross the pole and pick up the contribution from $H_{0}^{p}$ which, for $\Phi>0$ is the reflection of the incident wave, and for $\Phi<0$ is a cancellation of the incident wave, i.e a shadow region. The regions determined by the reflection/shadow are bounded by angles which generate the discontinuity in the standard geometric optic far-field solution, (1.2.1.4). Thus we must construct a uniformly-valid expansion about these boundaries which are then seen as Fresnel regions emanating from the nose of the aerofoil. To evaluate the behaviour in these regions further we must expand about the pole and saddle point, and consider an integration contour that is a small line segment tangent to the steepest descent contour at the point of stationary phase (in the direction of steepest descent). Near to the saddle point, $\lambda=\lambda_{0}=-w \cos \theta$, we can approximate 


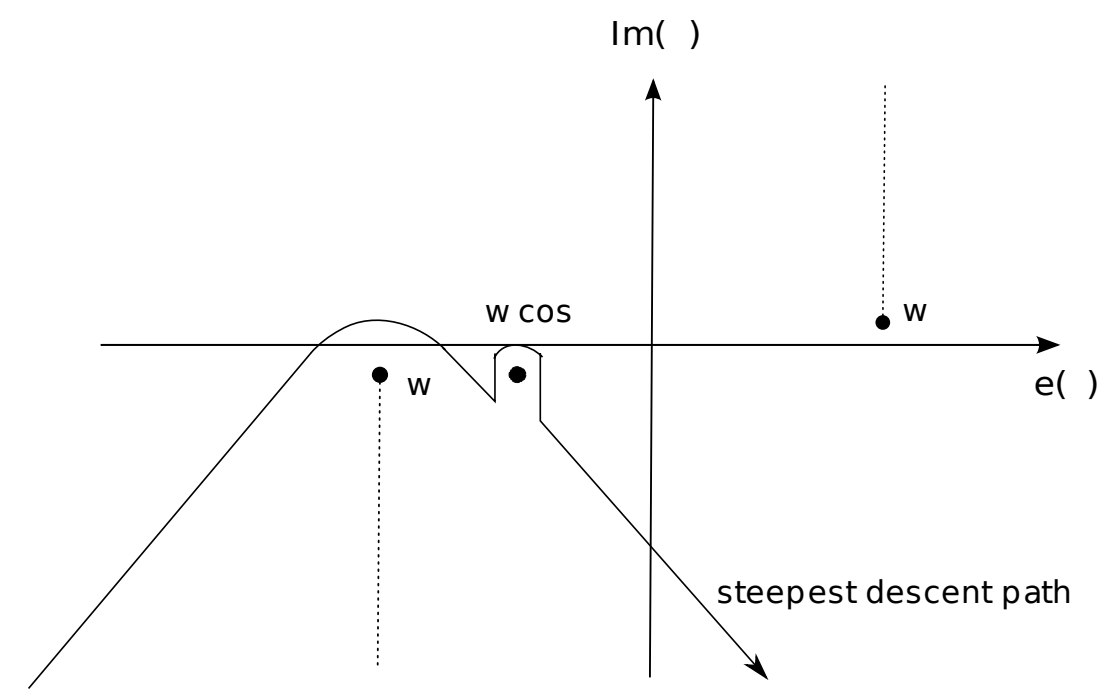

Figure 1.2: Steepest descents contour for phase function $-a(\lambda)$ in the $\lambda$-plane.

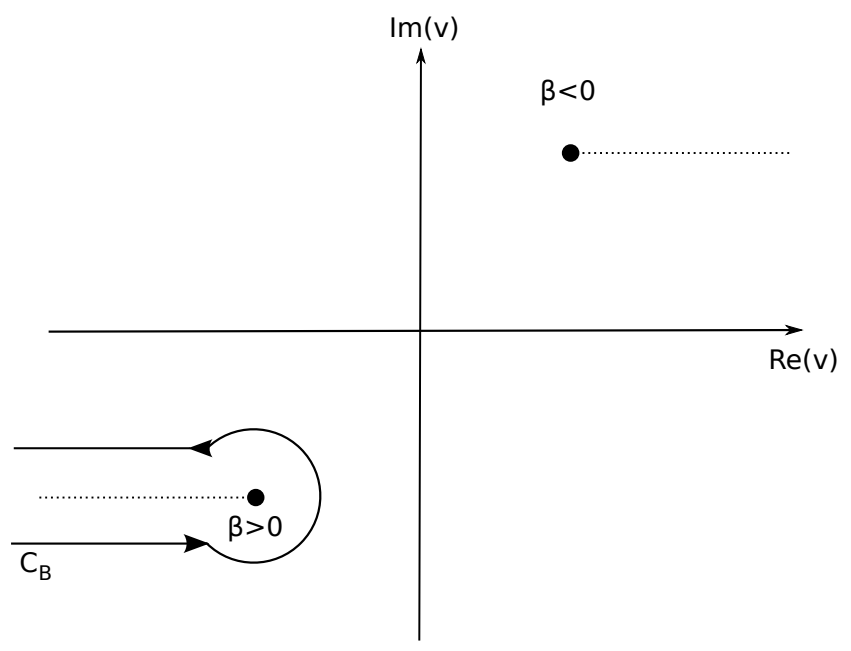

Figure 1.3: Location of the branch cut of $\sqrt{v-\beta \mathrm{e}^{-3 \pi \mathrm{i} / 4}}$ in the $v$-plane, and contour $C_{B}$. 
the phase function by a Taylor series, hence using the change of variables

$$
\lambda+w \cos \theta=\left(\frac{2 w \sin ^{2} \theta}{R}\right)^{1 / 2} \mathrm{e}^{-\pi \mathrm{i} / 4} v
$$

we can write

$$
H_{0}=-\frac{\mathrm{i} \sin \chi \operatorname{sgn}(\psi) \mathrm{e}^{\mathrm{i} k w r}}{2 \pi \sqrt{\cos \chi+1} \sqrt{(1-\cos \theta)}} \int_{C^{\prime}} \frac{\mathrm{e}^{-v^{2}} d v}{v-\beta \mathrm{e}^{-3 \pi \mathrm{i} / 4}},
$$

where

$$
\beta=(\cos \chi-\cos \theta) \sqrt{\frac{w R}{2 \sin ^{2} \theta}},
$$

and $C^{\prime}$ is the straight line from $-\infty \mathrm{e}^{\pi \mathrm{i} / 4}$ to $\infty \mathrm{e}^{\pi \mathrm{i} / 4}$. There are now two distinct limits, namely $\beta=O(1)$ and $\beta \gg 1$. For $\beta \gg 1$ we return to the original limit, whereas for $\beta=O(1)$, consider

$$
I=\int_{-\infty}^{\infty} \frac{\mathrm{e}^{-v^{2} x}}{v-\beta \mathrm{e}^{-3 \pi \mathrm{i} / 4}} d v
$$

By solving a first order PDE for $I(x)$ and ensuring that when we deform the contour $C^{\prime}$ to the real axis to give (1.2.1.8) we go around the pole and include its residue, we find that

$$
H_{0}{ }^{u} \sim \frac{-\sin (\chi) \operatorname{sgn}(\psi) \mathrm{e}^{\mathrm{i} k w r} \mathrm{e}^{-\mathrm{i} \beta^{2}}}{2 \sqrt{1+\cos \chi} \sqrt{1-\cos \theta}} \operatorname{sgn}(\beta) \operatorname{erfc}\left[\mathrm{e}^{-\pi \mathrm{i} / 4}|\beta|\right]+H_{0}^{p},
$$

This is continuous in $\theta$, and now has no singularities; we note in particular that the square root term, $(1-\cos \theta)^{-1 / 2}$, does not cause any problems as $\theta \rightarrow 0$ since the expansion of the complementary error function as $\beta \rightarrow \infty$ removes the singularity. The uniformly-valid solution consists of two different acoustic forms, the first term in (1.2.1.9) represents scattering of the incident sound wave by interaction with the edge of the flat plate and away from $\cos \theta=\cos \chi$ takes a cylindrically decaying form, whilst the second term represents direct blocking and reflection of the incident sound wave, and takes the form of a plane wave.

\subsubsection{Solution for the Thickness-Related Term $H_{1}$}

At this order, we begin to take account of the thickness of the aerofoil. We have separated the leading-order correction term in (1.2.0.3) so that we may apply different physical interpretations to each term, and solve the problem more easily. $H_{1}$ is therefore chosen to represent only the effect of thickness on the boundary condition, and accounts for the (thickness-dependent) evolution of the phase of the incident sound wave. Specifically, 
$H_{1}$ satisfies

$$
\begin{aligned}
D\left(H_{1}\right) & =0 \\
\left.\frac{\partial H_{1}}{\partial \Psi}\right|_{\substack{\Phi>0 \\
\Psi=0 \pm}} & =-\frac{\mathrm{i} w V(\chi) \operatorname{sgn}(\Psi) a \mathrm{e}^{\mathrm{i} w \cos (\chi) \Phi}}{4 \beta_{\infty} \cos (\chi) \sqrt{\Phi}} .
\end{aligned}
$$

The boundary condition, $(1.2 .2 .1 \mathrm{~b})$, arises from the $O\left(\epsilon k^{1 / 2}\right)$ correction in the boundary condition generated only by the incident sound wave, i.e. by the term $\frac{\partial h_{I}}{\partial \psi}$ in $(1.2 .0 .2 \mathrm{~b})$.

Equation (1.2.2.1) has solution

$$
H_{1}=\frac{\mathrm{e}^{\mathrm{i} \pi / 4} \mathrm{i} w V(\chi) a}{8 \sqrt{\pi} \beta_{\infty} \cos \chi} \int_{-\infty}^{\infty} \frac{\mathrm{e}^{-\mathrm{i} \lambda \Phi-|\Psi| \sqrt{\lambda^{2}-w^{2}}}}{\sqrt{\lambda^{2}-w^{2}} \sqrt{\lambda+w \cos \chi}} d \lambda .
$$

Using the method of steepest descents, we find that in terms of outer variables,

$$
H_{1} \sim L_{1}(\theta) \frac{\mathrm{e}^{\mathrm{i} k w r}}{\sqrt{k r}}+O\left(k^{-3 / 2}\right)
$$

where

$$
L_{1}=-\frac{V(\chi) a}{4 \sqrt{2} \beta_{\infty} \cos \chi \sqrt{\cos \chi-\cos \theta}} .
$$

The non-uniformity in $L_{1}(\theta)$ at the points $\cos \chi=\cos \theta$ is caused by the collision of the branch point (as opposed to a pole) at $\lambda=-w \cos \chi$ and the saddle point, $\lambda_{0}=-w \cos \theta$, and hence the uniformly-valid contribution differs from the usual Fresnel region solution (caused by the collision of a pole and the saddle point) that was demonstrated in the $H_{0}$ solution. Rather than picking up a residue contribution as we deform to the path of steepest descents (such as $H_{0}^{p}$ from (1.2.1.5)), we wrap the steepest descents contour around the branch cut between $-w \cos \chi$ and infinity. The branch cut dominates the integral for $\cos \theta$ close to $\cos \chi$ since here the point of stationary phase coalesces with the branch point.

To evaluate the branch cut contribution, we apply the same change of variables, (1.2.1.6), to the original integral expression of $H_{1}$, given by (1.2.2.2), to obtain

$$
H_{1}^{u} \sim c(\theta) \int_{C^{\prime \prime}} \frac{\mathrm{e}^{-v^{2}}}{\sqrt{v-\beta \mathrm{e}^{-3 \pi \mathrm{i} / 4}}} d v \equiv c(\theta) I,
$$

where $\beta$ is still defined by (1.2.1.7b). For $\beta=O(1)$, the contour $C^{\prime \prime}$ is wrapped around the branch cut at $\beta \mathrm{e}^{-3 \pi \mathrm{i} / 4}$, i.e. is given by $C_{B}$ shown in Figure 1.2 when $\beta>0$. For 


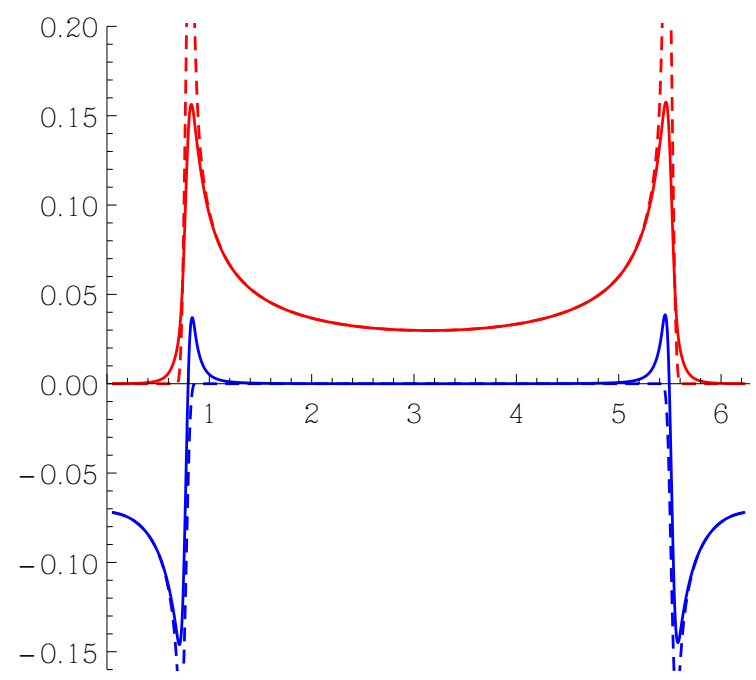

Figure 1.4: Comparison of real (red) and imaginary (blue) parts of the uniformly-valid (solid) and non uniformly-valid (dashed) far-field approximations for the directivity of $H_{1}$, as functions of $\theta$, at $k r=100$.

$|\beta| \gg 1$, the contour, $C^{\prime \prime}$ is the standard contour we would obtain via the method of steepest descents (because there is no coalescence of the saddle point and the branch point), which is the contour from $-\infty$ to $\infty$ along the real axis. Therefore for $|\beta| \gg 1$ we return to the original limit, (1.2.2.3b).

For $\beta>0$, evaluating the resulting integral around $C_{B}$, referring to Abramowitz \& Stegun (1964, p.687), yields

$$
I=\int_{C_{B}} \frac{\mathrm{e}^{-v^{2}}}{\sqrt{v-\beta \mathrm{e}^{-3 \pi \mathrm{i} / 4}}} d v=\sqrt{-\beta \mathrm{e}^{-3 \pi \mathrm{i} / 4}} \mathrm{i} K_{1 / 4}\left(\beta^{2} \mathrm{i} / 2\right) \mathrm{e}^{\mathrm{i} \beta^{2} / 2},
$$

where $K_{1 / 4}(x)$ is a modified Bessel function of the second kind. For $\beta<0$ we reverse the sign of $v$ within the integral expression for $I$ given in (1.2.2.5), and proceed as before to obtain a similar result.

Therefore the uniformly-valid expansion of $H_{1}$ is

$$
H_{1}^{u}=-\frac{\mathrm{e}^{\pi \mathrm{i} / 4} \sqrt{w} V(\chi) a \operatorname{sgn}(\beta) \sqrt{\cos \chi-\cos \theta}}{8 \beta_{\infty} \cos \chi \sqrt{\pi}|\sin \theta|} K_{1 / 4}\left(\beta^{2} \mathrm{i} / 2\right) \mathrm{e}^{\mathrm{i} k w r} \mathrm{e}^{\mathrm{i} \beta^{2} / 2}
$$

In the limit of large $\beta$ this returns to the initial far-field expansion, (1.2.2.3b). For $k r=100$, Figure 1.4 illustrates the uniformly-valid expression versus the non-uniformly valid solution. A discussion of this type of uniformly-valid solution can be found in Bleistein \& Handelsman (1975). 


\subsubsection{Solution for the Thickness-Related Term $\mathrm{H}_{2}$}

Tsai (1992) constructs $H_{2}$ as the solution that arises due to a source term in the governing equation. We do not have such a source term in (1.2.0.2a), because the incident sound wave does not create any upstream vortical perturbations in our linearised system (an incident gust does of course produce upstream vorticity), hence there is no need for the $H_{2}$ term as defined by Tsai (1992). We do, however, have an awkward boundary condition arising from the non-uniform flow around the nose of the aerofoil. We let $\mathrm{H}_{2}$ be the function that solves for this, i.e. $\mathrm{H}_{2}$ accounts for the distortion of the mean flow around the aerofoil nose, so

$$
D\left(H_{2}\right)=0,\left.\quad \frac{\partial H_{2}}{\partial \Psi}\right|_{\substack{\Phi>0 \\ \Psi=0 \pm}}=\frac{-M_{\infty}^{2}}{t^{\prime} \sqrt{k}} \frac{\partial q}{\partial \Psi}(\Phi, 0) \mathrm{e}^{\mathrm{i} w \cos (\chi) \Phi}
$$

This boundary condition arises from the $O\left(t k^{1 / 2}\right)$ correction in (1.2.0.2b) generated by the interaction of the incident sound wave with the mean flow; the second term on the right hand side of (12.0.2b). Again we solve using the Wiener-Hopf method, but this time, due to the boundary condition, the process is more involved. The solution is given by

$$
H_{2}=\frac{-\operatorname{sgn}(\Psi)}{2 \pi} \int_{-\infty}^{\infty} A_{+}(\lambda) \mathrm{e}^{-\mathrm{i} \lambda \Phi-|\Psi| \sqrt{\lambda^{2}-w^{2}}} d \lambda,
$$

where $A_{+}(\lambda)$ is to be determined from the Wiener-Hopf equation

$$
\sqrt{\lambda+w} A_{+}(\lambda)-F_{+}(\lambda)=\frac{D_{-}(\lambda)}{\sqrt{\lambda-w}}+F_{-}(\lambda)
$$

The $F_{ \pm}(\lambda)$ are determined via

$$
\frac{D_{+}(\lambda)}{\sqrt{\lambda-w}}=F_{+}(\lambda)+F_{-}(\lambda)
$$

with

$$
D_{+}(\lambda)=-\frac{M_{\infty}^{2}}{t^{\prime} \sqrt{k}} \int_{0}^{\infty} \frac{\partial q}{\partial \Psi}(x, 0) \Theta(x) \mathrm{e}^{\mathrm{i} w \cos \chi x} \mathrm{e}^{\mathrm{i} \lambda x} d x,
$$

and $\Theta(x)$ is the Heaviside step function. Recall a subscript \pm denotes a function that is analytic in the upper/lower half plane. $D_{-}$is an unknown function, given by

$$
D_{-}(\lambda)=\left.\int_{-\infty}^{0} \frac{\partial H_{2}}{\partial \Psi}\right|_{\Psi=0} \mathrm{e}^{\mathrm{i} \lambda \Phi} d \Phi .
$$


To evaluate $A_{+}$(and therefore $H_{2}$ ) we refer to Jones (1966) and Tsai (1992). Tsai imposes physical Kutta conditions that $H\left(\Phi, 0_{ \pm}\right) \sim \pm \sqrt{\Phi}$ for small positive $\Phi$, and $\left.\frac{\partial H_{2}}{\partial \Psi}\right|_{\Psi=0} \sim(-\Phi)^{-1 / 2}$ for small negative $\Phi$. We impose the same conditions, therefore, by relating small $\Phi$ behaviour to large $\lambda$ behaviour, we know that

$$
A_{+}(\lambda) \sim \lambda^{-3 / 2}, \quad D_{+}(\lambda) \sim \lambda^{1 / 2}, \quad \text { and } \quad D_{-}(\lambda) \sim \lambda^{-1 / 2}
$$

as $\lambda \rightarrow \infty$ in the appropriate half planes. By considering (1.2.3.2c) we see that $F_{+}+F_{-} \sim$ const. as $\lambda \rightarrow \infty$. Due to the Kutta conditions imposed for small $\Phi$ values, we require $F_{+} \rightarrow 0$ as $\lambda \rightarrow \infty$, so this constant only lies within $F_{-}$. The left hand side of equation (1.2.3.2b) is analytic in the upper half plane, whilst the right hand side is analytic in the lower half plane. Both sides have a small region of overlap (allowed by giving $w$ a small positive imaginary part), and for large $\lambda$ both sides tend to a constant. Therefore both sides give an entire function, which by Liouville's theorem (Noble, 1998), must be a constant. We have therefore determined $A_{+}$up to a constant as

$$
A_{+}(\lambda)=\frac{F_{+}(\lambda)+\text { const. }}{\sqrt{\lambda+w}} .
$$

We can choose to insert the constant into the function $F_{+}$and write

$$
F_{+}(\lambda)=\frac{1}{2 \pi \mathrm{i}} \int_{C_{1}} \frac{D_{+}(\lambda)+C \sqrt{\lambda-w}}{\sqrt{\kappa-w}} \frac{d \kappa}{\kappa-\lambda},
$$

where $C_{1}$ is a contour running parallel to but just above the real axis, and below $w$. The constant, $C$, is determined by ensuring that $H_{2}$ behaves in agreement with our restrictions imposed on its small $\Phi$ behaviour.

We therefore choose $C=-M_{\infty}^{2} a \mathrm{i} \sqrt{\pi} \beta_{\infty}^{-1} \mathrm{e}^{\pi \mathrm{i} / 4}$ by consulting Jones (1966, p. 468 Ex. 28 ), and after taking the outer limit of (1.2.3.2a) using the method of steepest descents we obtain

$$
H_{2} \sim L_{2}(\theta) \frac{\mathrm{e}^{\mathrm{i} k w r}}{\sqrt{k r}}+O\left(k^{-3 / 2}\right)
$$

where

$$
\begin{aligned}
& L_{2}(\theta)=\frac{-a \operatorname{sgn}(\Psi) M_{\infty}^{2} \cos \theta / 2}{\beta_{\infty} \sqrt{\pi}}(\tilde{\gamma}+\log [(\cos \chi+1) w]+2-\pi \mathrm{i} \\
&\left.-2 \mathrm{i} \frac{\sqrt{(\cos \chi-\cos \theta)}}{\sqrt{(1+\cos \theta)}} \arcsin \left[\frac{\sqrt{(1+\cos \theta)}}{\sqrt{\cos \chi+1}}\right]\right),
\end{aligned}
$$


and $\tilde{\gamma}$ is the Euler-Mascheroni constant. Although it may appear that (1.2.3.2a) has a branch point colliding with the saddle point to produce a non-uniformity, as it did for $H_{1}$, in fact this is not the case because the function $F_{+}(\lambda)$ ensures regular behaviour at the saddle point. The solution presented for $L_{2}$ varies from that presented in Ayton \& Peake (2013), where the term $-\pi$ i was given as $-\pi \mathrm{i} / 2$.

This Wiener-Hopf method is slightly more complicated that the standard method used in Myers \& Kerschen (1997), Tsai (1992) and the previous sections here, because for those, $F_{ \pm} \rightarrow 0$ as $\lambda \rightarrow \infty$ due to differing boundary conditions, hence they effectively had $C=0$.

\subsubsection{Solution for the Thickness-Related Term $H_{3}$}

The contribution $\mathrm{H}_{3}$ arises from terms in (1.2.0.2a) that contain $\epsilon q(\phi, \psi)$, i.e. the effects of the non-uniform flow near the nose on the propagation of the sound through that region. The equation can be rearranged so that the interaction of $\epsilon q(\phi, \psi)$ is seen as an equivalent source term located at the leading edge; this is called a propagation source (Tsai, 1992), and so $H_{3}$ satisfies

$$
\begin{aligned}
& D\left(H_{3}\right)=-\frac{2 w^{2} \tilde{a} \beta_{\infty} \sin \theta / 2}{\sqrt{R}} H_{0}+\frac{(\gamma+1) M_{\infty}^{4} \tilde{a} \sin \theta / 2}{\beta_{\infty}^{3} \sqrt{R}}\left(\frac{\partial^{2} H_{0}}{\partial \Psi^{2}}+2 \mathrm{i} \delta \frac{\partial H_{0}}{\partial \Phi}\right. \\
&\left.+\left(w^{2}+\delta^{2}\right) H_{0}\right)+\frac{\tilde{a}(\gamma+1) M_{\infty}^{4} \sin 3 \theta / 2}{2 \beta_{\infty}^{3} R^{3 / 2}}\left(\frac{\partial H_{0}}{\partial \Phi}-\mathrm{i} \delta H_{0}\right), \\
&\left.\frac{\partial H_{3}}{\partial \Psi}\right|_{\substack{\Phi>0 \\
\Psi=0}}=\frac{M_{\infty}^{2} \tilde{a}}{2 \beta_{\infty} \Phi^{3 / 2}} H_{0},
\end{aligned}
$$

where $\tilde{a}=\operatorname{sgn}(\Psi) a$. This final boundary condition arises from the second terms on the left hand side of (1.2.0.2b). The particular solution to (1.2.4.1) is solved for in Appendix A, and has outer limit

$$
\begin{aligned}
H_{3 p} \sim & \frac{a \sqrt{w} \beta_{\infty} \sin \chi \mathrm{e}^{-\mathrm{i} \pi / 4} \sin \theta \mathrm{e}^{\mathrm{i} w k r}}{\sqrt{\pi} \sqrt{\cos \chi+1}(\cos \chi-\cos \theta)}\left(1-\frac{(\gamma+1) M_{\infty}^{4}}{2 \beta_{\infty}^{4} w^{2}}(\delta-w \cos \theta)^{2}\right) \\
& +L_{3 p}(\theta) \frac{\mathrm{e}^{\mathrm{i} w k r}}{\sqrt{k r}}+O\left(k^{-1}\right)
\end{aligned}
$$

where

$$
L_{3 p}(\theta)=\frac{-a \sin \chi(\gamma+1) M_{\infty}^{4}}{2 \sqrt{2} \beta_{\infty}^{3} w \sqrt{\cos \chi+1}}\left(\frac{w}{2} \sin 2 \theta-\delta \sin \theta\right) .
$$

We do not calculate a uniformly-valid solution for $H_{3 p}$, since the term creating the nonuniformity when $\cos \chi-\cos \theta=0$ is not present in the outer solution; it is only required 
for matching the leading-edge inner solution to the leading-edge outer solution.

We now seek a complementary solution, $H_{3 c}$, to (1.2.4.1a) such that $H_{3}=H_{3 p}+H_{3 c}$, with

$$
\begin{aligned}
D\left(H_{3 c}\right) & =0, \\
\left.\frac{\partial H_{3 c}}{\partial \Psi}\right|_{\substack{\Phi>0 \\
\Psi=0}} & =d(\Phi),
\end{aligned}
$$

where

$$
d(\Phi)=\frac{M_{\infty}^{2} a}{2 \beta_{\infty} \Phi^{3 / 2}} \mathrm{e}^{\mathrm{i} w \cos (\chi) \Phi} \operatorname{erf}\left[\mathrm{e}^{\pi \mathrm{i} / 4} \sqrt{(\cos \chi-1) w \Phi}\right]-\left.\frac{\partial H_{3 p}^{*}}{\partial \Psi}\right|_{\substack{\Phi>0 \\ \Psi=0}}
$$

The first term in (1.2.4.3c) arises from the uniform expansion of $H_{0},(1.2 .1 .9)$, in the original boundary condition $(1.2 .4 .1 \mathrm{~b})$, while the second term is introduced to cancel the normal velocity of the particular solution $H_{3 p}$ and is defined in Appendix A. The full expansion for $d(\Phi)$ is given in Appendix $\mathrm{B}$, written in the form

$$
d(\Phi)=d_{1}(\Phi)+d_{2}(\Phi)+d_{3}(\Phi)
$$

so as to simplify later calculations. We set

$$
H_{3 c}=\Sigma_{i=1}^{3} H_{3 c_{i}}
$$

where each $H_{3 c_{i}}$ corresponds to the term $d_{i}$ in the boundary condition (1.2.4.3b). The solution to (1.2.4.3) can be obtained using the Wiener-Hopf method, giving

$$
H_{3 c_{i}}=-\frac{\operatorname{sgn}(\Psi)}{2 \pi} \int_{-\infty}^{\infty} F_{+i}(\lambda) \frac{\mathrm{e}^{a(\lambda, \Phi, \Psi)}}{\sqrt{\lambda+w}} d \lambda
$$

where the $F_{+i}$ are also given in Appendix B. In the limit of large $R$, we obtain

$$
H_{3 c_{i}} \sim L_{3 c_{i}}(\theta) \frac{\mathrm{e}^{\mathrm{i} w k r}}{\sqrt{k r}}+O\left(k^{-3 / 2}\right), \quad \text { for } i=1,2,3,
$$

where the expressions for the $L_{3 c_{i}}$, and their uniformly-valid counterparts, can be found in Appendix B. 


\subsubsection{Solution for the Camber-Related Terms $P_{i}, i=1,2,3$}

Recall that the $P_{i}$ terms in (1.2.0.3) are the components of the inner solution that correspond to the leading-edge effects of camber and angle of attack on the scattering of the incident sound wave. The $P_{1}$ solution comes from the differentiation of the integral in the exponent of $h_{I}$, given by (1.1.3.7), within the boundary condition (i.e. the effect of the cumulative distortion of the sound wave as it propagates through the non-uniform mean flow on the incident normal velocity) and so satisfies

$$
\begin{aligned}
D\left(P_{1}\right) & =0 \\
\left.\frac{\partial P_{1}}{\partial \Psi}\right|_{\substack{\Phi>0 \\
\Psi=0 \pm}} & =-\frac{\mathrm{i} w V(\chi) \operatorname{sgn}(\Psi) \mathrm{e}^{\mathrm{i} w \cos \chi \Phi}}{2 \sqrt{2} \beta_{\infty} \sin \chi \sqrt{\Phi}} .
\end{aligned}
$$

Again, using the Wiener-Hopf technique in a standard way, we find that

$$
P_{1}=\frac{\mathrm{e}^{\mathrm{i} \pi / 4} \mathrm{i} w V(\chi)}{4 \sqrt{2 \pi} \beta_{\infty} w \sin \chi} \int_{-\infty}^{\infty} \frac{\mathrm{e}^{-\mathrm{i} \lambda \Phi-|\Psi| \sqrt{\lambda^{2}-w^{2}}}}{\sqrt{\lambda^{2}-w^{2}} \sqrt{\lambda+w \cos \chi}} d \lambda,
$$

and a uniformly-valid outer limit can be found using the method described in Section 1.2 .2 , giving

$$
P_{1}^{u}=-\frac{\mathrm{e}^{\pi \mathrm{i} / 4} \sqrt{w} V(\chi) \operatorname{sgn}(\beta) \sqrt{\cos \chi-\cos \theta}}{4 \sqrt{2 \pi} \beta_{\infty} \sin \chi|\sin \theta|} K_{1 / 4}\left(\beta^{2} \mathrm{i} / 2\right) \mathrm{e}^{\mathrm{i} k w r} \mathrm{e}^{\mathrm{i} \beta^{2} / 2} .
$$

The $H_{2}$ term in (1.2.0.3) arose from the influence of the thickness-dependent part of the mean velocity potential in the boundary condition (1.2.0.2b) (i.e. the term containing $q$ ), however the camber-dependent part of the potential tells us that this term is zero, and hence we set $P_{2}=0$.

The term $P_{3}$ in (1.2.0.3) arises from the terms involving $q$ in (1.2.0.2a) forming a source term in a similar way to the propagation source for $H_{3}$, namely

$$
\begin{aligned}
& D\left(P_{3}\right)=\frac{\sqrt{2}}{\beta_{\infty} \sqrt{R}} \cos \frac{\theta}{2}\left(2 \beta_{\infty}^{2} w^{2} H_{0}-\frac{(\gamma+1) M_{\infty}^{4}}{\beta_{\infty}^{2}}\left[\frac{\partial^{2} H_{0}}{\partial \Psi^{2}}+2 \mathrm{i} \delta \frac{\partial H_{0}}{\partial \Phi}\right.\right. \\
& \left.\left.\quad+\left(w^{2}+\delta^{2}\right) H_{0}\right]\right)-\frac{1}{R^{3 / 2}} \cos \frac{3 \theta}{2} \frac{(\gamma+1) M_{\infty}^{4}}{\sqrt{2} \beta_{\infty}^{3}}\left[\frac{\partial H_{0}}{\partial \Phi}-\mathrm{i} \delta H_{0}\right] \\
& \left.\frac{\partial P_{3}}{\partial \Psi}\right|_{\substack{\Phi>0 \\
\Psi=0 \pm}}=0 .
\end{aligned}
$$

The boundary condition is zero normal derivative since the $\Psi$ derivative of the part of $q$ corresponding to the leading-edge camber and angle of attack is zero on the aerofoil. 
Hence we can use a modified form of the solution for the gust case from Myers \& Kerschen (1997); setting $P_{3}=P_{3 c}+P_{3 p}+P_{3 e}$, gives

$$
\begin{aligned}
& P_{3 p}(R, \theta)=\mathrm{i} w \sqrt{2 k r}\left(-2 \beta_{\infty}+\frac{(\gamma+1) M_{\infty}^{4}}{\beta_{\infty}^{3} w^{2}}(\delta-w \cos \theta)^{2}\right) \cos \frac{\theta}{2} H_{0} \\
& P_{3 c}(R, \theta)=\frac{\mathrm{i} w \sin \chi \beta_{\infty} \mathrm{e}^{\pi \mathrm{i} / 4}}{\sqrt{2 \pi}} \int_{-\infty}^{\infty}\left(\frac{1}{\sqrt{\lambda+\delta}}-\frac{\delta}{(\lambda+\delta)^{3 / 2}}\right) \frac{\mathrm{e}^{a(\lambda, \phi, \psi)}}{\sqrt{\lambda^{2}-w^{2}}} d \lambda, \\
& P_{3 e}(R, \theta)=\frac{-\sqrt{2 \pi}(\gamma+1) M_{\infty}^{2} \mathrm{i} w \sin \chi \mathrm{e}^{-\mathrm{i} \pi / 4}}{8 \beta_{\infty}^{3} \sqrt{\delta+w}}\left(H_{2}^{(1)}(w R) \cos 2 \theta+\frac{2 \mathrm{i} \delta}{w} H_{1}^{(1)}(w R) \cos \theta\right) .
\end{aligned}
$$

The modification to Myers and Kerschen's result arises in the first factor within the integrand of $P_{3 c}$. The outer limit of $P_{3 c}+P_{3 e}$ is

$$
P_{3 c}+P_{3 e} \sim D_{3}(\theta) \frac{\mathrm{e}^{\mathrm{i} k w r}}{\sqrt{k r}}+O\left(k^{-3 / 2}\right)
$$

where

$$
D_{3}(\theta)=\frac{w^{3 / 2} \beta_{\infty} \sin \chi \cos \theta}{(\delta-w \cos \theta)^{3 / 2}}-\frac{(\gamma+1) M_{\infty}^{4} \sin \chi}{\sqrt{w} \sqrt{\delta+w} \beta_{\infty}^{3}}\left(\frac{\delta}{2} \cos \theta-\frac{w}{4} \cos 2 \theta\right) .
$$

We have thereby now completed our asymptotic solution in the inner region, and have crucially found a uniformly-valid outer limit which can be matched onto the solution in the outer region.

\subsection{Solution for the Leading-Edge Outer and Transi- tion Regions}

We first solve for the boundary condition, (1.1.3.8), in the outer region. Clearly, $-h_{I}$ is a solution in the outer region, however we need to construct an outer solution, $h^{o}$, that matches to an appropriate function in the leading-edge inner limit, but also cancels the normal velocity of $h_{I}$ on the aerofoil surface. The appropriate function for $\psi>0$ is

$$
h^{o}=\mathrm{e}^{\mathrm{i} w k \cos \chi \phi+\mathrm{i} w k \sin \chi|\psi|+\mathrm{i} k w \epsilon \sigma_{1}(\tilde{\xi}, \tilde{\eta})}\left(\Theta\left(\theta_{t}\right)-\Theta\left(\theta_{t}-\chi\right)-\Theta(\theta)+\Theta(\theta-\chi)\right)
$$

where $\sigma_{1}$ is defined in (1.1.3.5), and $\tilde{\xi}=\phi+\cot \chi|\psi|, \tilde{\eta}=\phi-\cot \chi|\psi|$. The Heaviside step functions, $\Theta$, dictate that $h^{o}$ is only non-zero if an observer is in the direct shadow which is defined by the polar angle measured from the leading edge satisfying $\theta \in(0, \chi)$, 
and the polar angle measured from the trailing edge satisfying $\theta_{t} \in(0, \chi)$. For $\psi<0$ we require a reflected solution given by

$$
\begin{aligned}
h^{o}=-\mathrm{e}^{\mathrm{i} w k \cos \chi \phi+\mathrm{i} w k \sin \chi|\psi|+\mathrm{i} k w \epsilon \sigma_{1}(\tilde{\xi}, \tilde{\eta})}\left[\Theta\left(-\theta_{t}\right)\right. & -\Theta\left(-\theta_{t}-\chi\right) \\
& -\Theta(\theta-(2 \pi-\chi))+\Theta(\theta-2 \pi)] .
\end{aligned}
$$

In the far field, these regions reduce to a small, $O(1 / r)$, polar regions near $\theta=\chi, 2 \pi-\chi$, which coincide with the location of the shadow and reflection boundaries around which the Fresnel regions are centred. In the leading-edge inner limit, the terms from the $(-\Theta(\theta)+\Theta(\theta-\chi))$ contributions match to the outer limit of the inner leading-edge term, $H_{0}^{p}$, given by (1.2.1.5). The other terms, from the $\Theta\left(\theta_{t}\right)-\Theta\left(\theta_{t}-\chi\right)$ contributions, will match to a trailing-edge inner solution which comes from another pole contribution.

At distances of $r=O(k)$ in the far field, the scattered Fresnel regions emanating from the leading and trailing edges overlap and eclipse this $h^{o}$ term, similar to the case seen in Peake \& Kerschen (2004), thus in the far field we do not actually see $h^{o}$.

\subsubsection{Leading-Edge Ray Field}

The leading-edge ray field (which is a scattered solution) is analogous to the gust cases found in Tsai (1992) and Myers \& Kerschen (1997), so we propose a cylindrically decaying leading-edge outer solution of the form

$$
h_{l}=\frac{K_{0}(\theta)+t \sqrt{k} K_{1}(\theta)+\alpha_{\text {eff }} \sqrt{k} K_{2}(\theta)}{\sqrt{k r}} \mathrm{e}^{\mathrm{i} k w \sigma_{l}(r, \theta)} .
$$

We substitute (1.3.1.1a) into (1.1.2.4) to give the eikonal equation for $\sigma_{l}$, and solving up to and including $O(\epsilon)$, we obtain

$$
\sigma_{l}=r+\epsilon \sigma_{l_{1}}+O\left(\epsilon^{2}\right)
$$

where

$$
\sigma_{l_{1}}=V(\theta) \int_{0}^{r} q\left(r^{\prime}, \theta\right) d r^{\prime}+\sigma_{1}(0,0)
$$

and $V$ is defined in (1.1.3.6). We finally obtain the solutions to $K_{i}$ by Van Dyke's matching rule (Van Dyke, 1975), whereby we match the outer limit $(R \rightarrow \infty)$ of the inner solution (1.2.0.3) onto the inner limit $(r \rightarrow 0)$ of the outer solution (1.3.1.1a). 
Hence, similarly to Myers \& Kerschen (1997) and Tsai (1992),

$$
\begin{aligned}
& K_{0}(\theta)=L_{0}(\theta), \\
& K_{1}(\theta)=L_{1}(\theta)+L_{2}(\theta)+L_{3 p}(\theta)+L_{3 c_{1}}(\theta)+L_{3 c_{2}}(\theta)+L_{3 c_{3}}(\theta), \\
& K_{2}(\theta)=D_{1}(\theta)+D_{2}(\theta)+D_{3}(\theta),
\end{aligned}
$$

in the non uniformly-valid case. The cross-term from the inner limit of $h_{l}$, given by $K_{0}(\theta) \mathrm{i} k w \epsilon V(\theta) \int_{0}^{r} q d r^{\prime}$, matches to the first term in the particular solution, $H_{3 p}$ from (1.2.4.2a), and to $P_{3 p}$ from (1.2.5.3a). For a uniformly-valid matching, we must replace the non uniformly-valid terms, $\left(L_{0}, L_{1}, L_{3 c_{1}}, L_{3 c_{2}}, D_{1}\right)$ by their uniformly-valid counterparts (e.g. $H_{0}^{u} \sqrt{k r}$ ). This still provides a matching, however the amplitude function is now dependent on $r$ as well as on $\theta$. In all cases that follow, to obtain the uniformly-valid solution we replace any non uniformly-valid terms by their uniformly-valid counterparts in this way. To aid the understanding of the matching problems, results will in general be given in terms of non uniformly-valid solutions where appropriate.

\subsubsection{Leading-Edge Transition Solution}

When finding the leading-edge outer scattered solution we did not impose a boundary condition on the aerofoil surface, and furthermore the expansion of $L_{3 c_{2}}$ becomes non uniform at small angles (illustrated by the logarithmic singularity in (B.8) at $\lambda=-w$ ). These two issues lead us to look for a boundary-layer style solution, which emanates from the leading edge and is valid close to the aerofoil surface, that cancels the normal velocity generated by the leading-edge outer ray field. When $\theta=O\left(k^{-1 / 2}\right)$, and $r=O(1)$, there is a distinguished limit in the integral term in $L_{3 c_{2}}^{u}$, (B.10), giving a boundary-layer style structure to our leading-edge inner solution. We therefore seek a complementary solution, $h_{l s}$, that is exponentially small outside a region of width $O\left(k^{-1 / 2}\right)$ on the aerofoil surface. In this case, the matching previously done of $H$ onto $h_{l}$ is still valid, and we can write the total leading-edge solution as

$$
h_{l}^{u}=h_{l}+h_{l s}
$$

We do, however, require an explicit expression for this transition solution in order to evaluate the pressure jump it creates at the trailing edge of the aerofoil. We then also require a suitable complementary function around the trailing edge to ensure the pressure is continuous across the wake, i.e the Kutta condition is satisfied.

We know that $h_{l s}$ must still satisfy governing equation, (1.1.2.4), and it must have 
boundary condition

$$
\frac{\partial h_{l s}}{\partial \psi}+\left.M_{\infty}^{2} \epsilon \frac{\partial q}{\partial \psi} h_{l s}\right|_{\psi=0_{ \pm}}=-\frac{\partial h_{l}}{\partial \psi}-\left.M_{\infty}^{2} \epsilon \frac{\partial q}{\partial \psi} h_{l}\right|_{\psi=0_{ \pm}}
$$

to ensure zero normal velocity on both upper and lower blade surfaces. On the upper surface we introduce a rescaled variable $\eta=\sqrt{k} \psi$ to represent this boundary layer structure. We can once again consider the thickness-dependent terms and camber-dependent terms separately, denoting the thickness-dependent part of the mean velocity perturbation, $\epsilon q$, as $\epsilon q^{(t)}$ and similarly the camber-dependent part as $\epsilon q^{(c)}$. We begin with the thickness dependent terms hence follow the method set out by Tsai (1992). By balancing terms in the boundary condition (1.3.2.2), we consider a solution of the form

$$
h_{l s}^{(t)}(\phi, \eta)=t \mathcal{G}(\phi, \eta) \mathrm{e}^{\mathrm{i} k w \phi+\mathrm{i} k w \epsilon V(0) \int_{0}^{r} q\left(r^{\prime}, 0\right) d r^{\prime}+\mathrm{i} k w \epsilon \sigma_{1}(0,0)},
$$

so to leading order, (1.1.2.4) becomes

$$
2 \mathrm{i} w \frac{\partial \mathcal{G}}{\partial \phi}+\frac{\partial^{2} \mathcal{G}}{\partial \eta^{2}}=0
$$

The boundary condition is applied along the whole of $\phi>0$; we deduct the $\phi>2$ part that we don't require later in Sections 1.4.2 and 1.4.3 as part of the trailing-edge transition solution. We therefore require

$$
\left.\frac{\partial \mathcal{G}}{\partial \eta}\right|_{\eta=0}=b(\phi)
$$

where

$$
b(\phi)=\frac{\mathrm{e}^{-\mathrm{i} \pi / 4} V(0)}{\sqrt{\pi} \beta_{\infty} \sqrt{\cos \chi+1}} \frac{\sqrt{w} \sin \chi}{1-\cos \chi} \frac{1}{\phi}\left(\frac{\beta_{\infty}}{t^{\prime} \sqrt{\phi}} \int_{0}^{\phi} \frac{\partial q^{(t)}}{\partial \theta}\left(r^{\prime}, 0\right) d r^{\prime}+a\right)
$$

for $0<\phi<2$, and

$$
b(\phi)=\frac{\mathrm{e}^{-\mathrm{i} \pi / 4} V(0)}{\sqrt{\pi} \beta_{\infty} \sqrt{\cos \chi+1}} \frac{\sqrt{w} \sin \chi}{1-\cos \chi} \frac{1}{\phi}\left(\frac{\beta_{\infty}}{t^{\prime} \sqrt{\phi}} \int_{0}^{2} \frac{\partial q^{(t)}}{\partial \theta}\left(r^{\prime}, 0\right) d r^{\prime}+a\right)
$$

for $\phi>2$.

By using the Laplace transform with respect to the variable $\eta$ in (1.3.2.4a) and 
applying the required boundary condition, we find that

$$
\mathcal{G}(\phi, \eta)=\frac{V(0) \sin \chi}{\sqrt{2} \beta_{\infty} \pi \sqrt{\cos \chi+1}(\cos \chi-1)} \int_{0}^{\phi}\left[\frac{a}{\xi}+\frac{\beta_{\infty}}{t^{\prime} \xi^{3 / 2}} \int_{0}^{\xi} \frac{\partial q^{(t)}}{\partial \theta}\left(r^{\prime}, 0\right) d r^{\prime}\right] \frac{\mathrm{e}^{\mathrm{i} \eta^{2} w / 2(\phi-\xi)}}{\sqrt{\phi-\xi}} d \xi
$$

for $0<\phi<2$, and

$$
\begin{aligned}
\mathcal{G}(\phi, \eta)= & \frac{V(0) \sin \chi}{\sqrt{2} \beta_{\infty} \pi \sqrt{\cos \chi+1}(\cos \chi-1)}\left(\int_{0}^{2}\left[\frac{a}{\xi}+\frac{\beta_{\infty}}{t^{\prime} \xi^{3 / 2}} \int_{0}^{\xi} \frac{\partial q^{(t)}}{\partial \theta}\left(r^{\prime}, 0\right) d r^{\prime}\right] \frac{\mathrm{e}^{\mathrm{i} \eta^{2} w / 2(\phi-\xi)}}{\sqrt{\phi-\xi}} d \xi\right. \\
& \left.+\int_{2}^{\phi}\left[\frac{a}{\xi}+\frac{\beta_{\infty}}{t^{\prime} \xi^{3 / 2}} \int_{0}^{2} \frac{\partial q^{(t)}}{\partial \theta}\left(r^{\prime}, 0\right) d r^{\prime}\right] \frac{\mathrm{e}^{\mathrm{i} \eta^{2} w / 2(\phi-\xi)}}{\sqrt{\phi-\xi}} d \xi\right)
\end{aligned}
$$

for $\phi>2$ (this will be used to find the pressure jump reaching the trailing edge). On the lower surface, $\psi=0_{-}$, we apply the same theory as for the upper surface, but using instead the transform variable $\eta=-\sqrt{k} \psi$. The camber-dependent transition solution is similar to that found in Myers \& Kerschen (1997) with appropriate redefinition of terms, so in the far-field limit takes the form

$$
\begin{gathered}
h_{l s}^{(c)} \sim \frac{\mathrm{e}^{\mathrm{i} k w \phi+\mathrm{i} k w \epsilon V(0) \int_{0}^{r} q\left(r^{\prime}, 0\right) d r^{\prime}+\mathrm{i} k w \epsilon \sigma_{1}(0,0)}}{\sqrt{r}} D_{l t r}(\theta), \\
D_{l t r}(\theta)=\operatorname{sgn}(\psi) \frac{\mathrm{e}^{3 \mathrm{i} \pi / 4} \epsilon V(0)}{\sqrt{2 w \pi} \beta_{\infty}} P_{l}\left(0_{ \pm}\right)\left[\int_{0}^{2} \mathrm{e}^{\mathrm{i} k w(1-\cos \theta) \xi}\left(\xi^{-1 / 2} y^{(c) \prime}(\xi)-\xi^{-3 / 2} y^{(c)}(\xi)\right) d \xi\right. \\
\left.+2^{3 / 2} y^{(c) \prime}(2)\left(\mathrm{e}^{2 \mathrm{i} k w(1-\cos \theta)}-\sqrt{\pi} e^{-\pi \mathrm{i} / 4} \sqrt{2 k w(1-\cos \theta)} \operatorname{erfc}\left(\mathrm{e}^{-\pi \mathrm{i} / 4} \sqrt{2 k w(1-\cos \theta)}\right)\right)\right] . \\
P_{l}(\theta)=\left[L_{0}(\theta)+\alpha_{\mathrm{eff}} \sqrt{k}\left(D_{1}(\theta)+D_{2}(\theta)+D_{3}(\theta)\right)\right] .
\end{gathered}
$$

The overall order of the camber-dependent transition solution is $O(\epsilon)$, like the thicknessdependent transition solution. We have now completed our description of the solution in the transition region. The total leading-edge transition solution (both thickness- and camber-dependent parts) is consistent with the leading-edge inner and outer solutions because in both limits the transition solution tends to zero except at small angles downstream (where $\theta \approx 0,2 \pi$ ) in the far-field limit. The difference at these small angles does not invalidate our current solutions because we correct these differences with trailing-edge solutions later.

We can now determine the total far-field sound scattered from the leading edge, given 


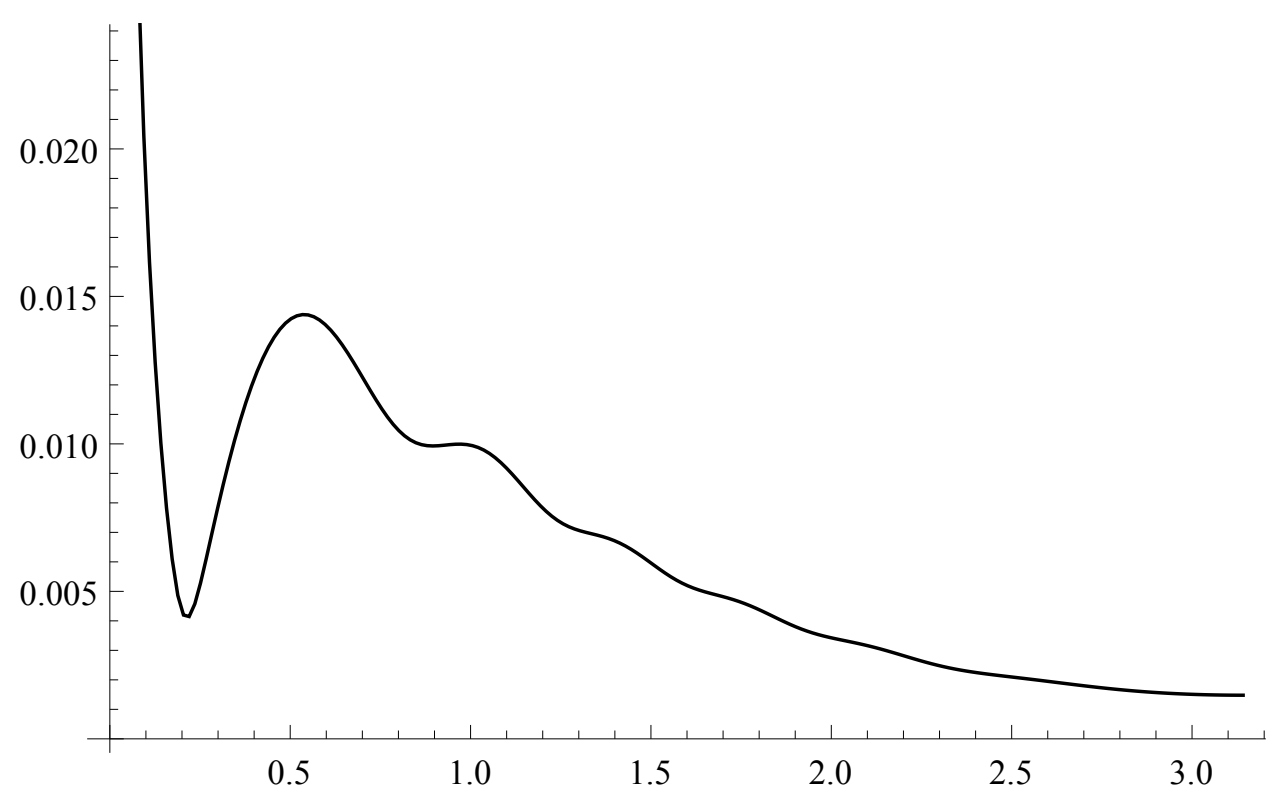

Figure 1.5: Magnitude of the far-field limit of the leading-edge transition solution (multiplied by $\sqrt{r}$ ) as a function of polar angle, $\theta$, for a NACA 1112 aerofoil with $k=10$.

by (1.3.2.1), which for large $r$ is

$$
\begin{aligned}
h_{l}^{u}(r, \theta) \sim & \frac{\mathrm{e}^{\mathrm{i} k w \sigma_{l}(r, \theta)}}{\sqrt{k r}}\left\{L_{0}(\theta)+t \sqrt{k}\left[L_{1}(\theta)+L_{2}(\theta)+L_{3 p}(\theta)+L_{3 c_{1}}(\theta)+L_{3 c_{2}}(\theta)\right.\right. \\
& +L_{3 c_{3}}(\theta)-\frac{\operatorname{sgn}(\psi) \sin \chi \beta_{\infty}}{\sqrt{2} \pi \sqrt{\cos \chi+1}(\cos \chi-1)}\left(1-\frac{(\gamma+1) M_{\infty}^{4}}{2 \beta_{\infty}^{4} w^{2}}(\delta-w)^{2}\right) \\
& \left(\int_{0}^{2}\left[\frac{a}{\tau}+\frac{\beta_{\infty}}{t^{\prime} \tau^{3 / 2}} \int_{0}^{\tau} \frac{\partial q^{(t)}}{\partial \theta}\left(r^{\prime}, 0\right) d r^{\prime}\right] \mathrm{e}^{\mathrm{i} w k(1-\cos \theta) \tau} d \tau\right. \\
& \left.\left.+\int_{2}^{\infty}\left[\frac{a}{\tau}+\frac{\beta_{\infty}}{t^{\prime} \tau^{3 / 2}} \int_{0}^{2} \frac{\partial q^{(t)}}{\partial \theta}\left(r^{\prime}, 0\right) d r^{\prime}\right] \mathrm{e}^{\mathrm{i} w k(1-\cos \theta) \tau} d \tau\right)\right] \\
& \left.+\alpha_{\text {eff }} \sqrt{k}\left[D_{1}(\theta)+D_{2}(\theta)+D_{3}(\theta)\right]+\sqrt{k} D_{l t r}(\theta)\right\} .
\end{aligned}
$$

The outer limits of the transition solution has been used in (1.3.2.7). These outer limits only take effect at angles that are within $O\left(k^{-1 / 2}\right)$ of $\theta=0$, which can be seen by considering the small $\theta$ expansion of the phase function $k w(1-\cos \theta)$ in the transition terms in (1.3.2.6) and (1.3.2.7). Figure 1.5 illustrates the magnitude of the leading-edge transition solution. The relative size for comparison is $t=0.12$ which is the order of magnitude of the thickness-related perturbation terms to the leading-edge outer solution (multiplied by $\sqrt{r}$ ). We clearly see that away from $\theta=0$ the transition solution is negligible compared to the orders retained in our calculations. 


\subsection{Solution for the Trailing-Edge Outer, Transition, and Inner Regions}

\subsubsection{Trailing-Edge Ray Field}

Let $\left(\phi_{t}, \psi_{t}\right)$ be the potential-streamfunction coordinates with origin shifted to the trailing edge. Since we do not necessarily have a non-lifting aerofoil, $\phi_{t}$ changes above and below the aerofoil, so the transformation between leading- and trailing-edge coordinates is given by

$$
\phi=2 \pm \frac{\Gamma}{2}+\alpha_{t}+\phi_{t}, \quad \psi=\psi_{t},
$$

where $\Gamma$ is the total circulation around the aerofoil given by

$$
\begin{aligned}
\Gamma & =\frac{2 \pi}{\beta_{\infty}} \alpha_{g} \\
\alpha_{g} & =\alpha_{i}+\frac{1}{\pi} \int_{0}^{2} \frac{\epsilon y^{(c)}(x)}{\sqrt{x}(2-x)^{3 / 2}} d x
\end{aligned}
$$

and

$$
\alpha_{t}=\operatorname{Re}\left(\epsilon F^{(t)}(2)\right)
$$

where $F^{(t)}$ is the thickness-dependent part of the complex potential given by (1.1.1.7). Note that both $\Gamma$ and $\alpha_{t}$ are $O(\epsilon)$, given our assumption of thin aerofoils with small camber and angle of attack.

The ray field emanating from the trailing edge is of the same form as in the gust cases of Tsai (1992) and Myers \& Kerschen (1997), so we write

$$
h_{t}=\frac{K_{t}\left(\theta_{t}\right)}{k \sqrt{r_{t}}} \mathrm{e}^{\mathrm{i} k w \sigma_{t}\left(r_{t}, \theta_{t}\right)}
$$

where $\left(r_{t}, \theta_{t}\right)$ are trailing-edge polar coordinates, and the trailing-edge phase is

$$
\sigma_{t}=r_{t}+\epsilon \sigma_{t_{1}}+O\left(\epsilon^{2}\right)
$$

with

$$
\sigma_{t_{1}}=V\left(\theta_{t}\right) \int_{0}^{r_{t}} q\left(r_{t}^{\prime}, \theta_{t}\right) d r_{t}^{\prime}+g_{2}\left(\theta_{t}\right)
$$

The directivity function, $K_{t}\left(\theta_{t}\right)$, and the phase function, $g_{2}\left(\theta_{t}\right)$, are as yet unknown, and 
must be determined by matching with the trailing-edge inner solution.

\subsubsection{Trailing-Edge Inner Solution}

We move to the trailing-edge coordinate system (1.4.1.1). Letting $\left(\Phi_{t}, \Psi_{t}\right)=k\left(\phi_{t}, \psi_{t}\right)$ and $H_{t}{ }^{\text {tot }}\left(\Phi_{t}, \Psi_{t}\right)$ be the local inner solution for the trailing edge, the leading-order terms in (1.1.2.4) and (1.1.3.8) are

$$
\begin{gathered}
\frac{\partial^{2} H_{t}^{\text {tot }}}{\partial \Phi_{t}^{2}}+\frac{\partial^{2} H_{t}^{\text {tot }}}{\partial \Psi_{t}^{2}}+w^{2} H_{t}^{\text {tot }}=0, \\
\left.\frac{\partial H_{t}^{\text {tot }}}{\partial \Psi_{t}}\right|_{\substack{\Phi_{t}<0 \\
\Psi_{t}=0}}=-\left.\frac{\partial h_{I}}{\partial \psi}\right|_{\substack{\phi<0 \\
\psi=0}} .
\end{gathered}
$$

We require the pressure to be continuous across the wake $\psi_{t}=0, \phi_{t}>0$, where the pressure jump across the wake caused by our leading-edge transition solution is given in Appendix C. We can write the pressure jump as

$$
\left.p_{l}\right|_{\psi=0+}-\left.p_{l}\right|_{\psi=0-}=\Delta p\left(\phi_{t}\right) \sqrt{k} \mathrm{e}^{\mathrm{i} k\left(w-\delta M_{\infty}^{2}\right) \phi_{t}} \mathrm{e}^{\mathrm{i} k \epsilon w V(0)\left[\int_{0}^{\phi} q d r-\int_{0}^{2} q d r\right]},
$$

and later we separate this into contributions from thickness and camber, save for an interaction of the two in phase terms.

We first consider a trailing-edge inner solution, $H_{t}$, that cancels the pressure jump, (1.4.2.2), and satisfies (1.4.2.1a). With this solution we also enforce continuity of displacement across the wake,

$$
\left.\mathrm{e}^{-\mathrm{i} C_{+}} \frac{\partial H_{t}}{\partial \Psi_{t}}\right|_{\substack{\Phi_{t}>0 \\ \Psi_{t}=0+}}=\left.\mathrm{e}^{-\mathrm{i} C_{-}} \frac{\partial H_{t}}{\partial \Psi_{t}}\right|_{\substack{\Phi_{t}>0 \\ \Psi_{t}=0-}}
$$

where the constants $C_{ \pm}$are defined by

$$
C_{ \pm}=k \delta M_{\infty}^{2}\left(2 \pm \Gamma / 2+\alpha_{t}\right)
$$

and the continuity of pressure across the wake imposes

$$
\mathrm{e}^{-\mathrm{i} C_{-}}\left[\frac{\partial H_{t}}{\partial \Phi_{t}}-i \delta H_{t}\right]_{\substack{\Phi_{t}>0 \\ \Psi_{t}=0-}}-\mathrm{e}^{-\mathrm{i} C_{+}}\left[\frac{\partial H_{t}}{\partial \Phi_{t}}-\mathrm{i} \delta H_{t}\right]_{\substack{\Phi_{t}>0 \\ \Psi_{t}=0+}}=\frac{\Delta p(0)}{\sqrt{k}} \mathrm{e}^{\mathrm{i} w \Phi_{t}}
$$

We do not impose (1.4.2.1b) on $H_{t}$ since we introduce a further solution, $H_{t}^{\text {ref }}$, to account 
for the direct reflection of the incident sound wave by the trailing edge. We therefore require that $H_{t}$ has zero normal velocity on the aerofoil surface,

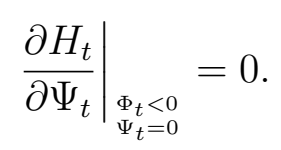

We solve for $H_{t}$ using the Wiener-Hopf method, to yield

$$
H_{t}=\frac{\operatorname{sgn}\left(\Psi_{t}\right) \mathrm{i} \sqrt{2 w} \Delta p(0) \mathrm{e}^{\mathrm{i} C_{ \pm}}}{4 \pi \sqrt{k}} \int_{-\infty}^{\infty} \frac{\mathrm{e}^{-\mathrm{i} \lambda \Phi_{t}-\left|\Psi_{t}\right| \sqrt{\lambda^{2}-w^{2}}}}{(\lambda+\delta)(\lambda+w) \sqrt{\lambda-w}} d \lambda
$$

We must match this solution onto the outer trailing-edge solution, (1.4.1.5), so evaluate (1.4.2.7a) for $R_{t} \gg 1$. The uniformly-valid expansion, found using Van der Waerden's method (Van der Waerden, 1952), is

$$
H_{t} \sim T\left(r_{t}, \theta_{t}\right) \frac{\mathrm{e}^{\mathrm{i} k w r_{t}}}{k \sqrt{r_{t}}}
$$

where

$$
\begin{array}{r}
T\left(r_{t}, \theta_{t}\right)=\frac{\operatorname{sgn}\left(\psi_{t}\right) \mathrm{i} \Delta p(0) \mathrm{e}^{\mathrm{i} C_{ \pm}}}{2(\delta-w)}\left[\operatorname{erfc}\left(\mathrm{e}^{-\mathrm{i} \pi / 4} \sqrt{w\left(1-\cos \theta_{t}\right) k r_{t}}\right) \mathrm{e}^{\mathrm{i} k w r_{t}\left(\cos \theta_{t}-1\right)} \sqrt{k r_{t}}\right. \\
\left.-\frac{\sqrt{2 w} \mathrm{e}^{\mathrm{i} \pi / 4}\left|\sin \theta_{t} / 2\right|}{\sqrt{\pi}\left(\delta-w \cos \theta_{t}\right)}\right],
\end{array}
$$

which is similar to the trailing-edge inner solution found by Tsai (1992).

The reflection of the incident sound wave by the aerofoil surface in the trailing-edge region requires us to construct the solution, $H_{t}^{\text {ref }}$, which we write as

$$
H_{t}^{r e f}=H_{0}^{t} \mathrm{e}^{\mathrm{i} k w \cos \chi\left(2 \pm \Gamma / 2+\alpha_{t}\right)+\mathrm{i} k w \epsilon \sigma_{1}^{t}(0,0)}
$$

where $\sigma_{1}^{t}$ is just $\sigma_{1}$, from (1.1.3.5), but in trailing-edge coordinates. To leading order, $H_{0}^{t}$ must satisfy

$$
\begin{aligned}
\frac{\partial^{2} H_{0}^{t}}{\partial \Phi_{t}^{2}}+\frac{\partial^{2} H_{0}^{t}}{\partial \Psi_{t}^{2}}+w^{2} H_{0}^{t} & =0 \\
\left.\frac{\partial H_{0}^{t}}{\partial \Psi_{t}}\right|_{\substack{\Phi_{t}<0 \\
\Psi_{t}=0 \pm}} & =-\mathrm{i} w \sin \chi \mathrm{e}^{\mathrm{i} w \cos \chi \Phi_{t}}
\end{aligned}
$$


Just as in Section 1.2.1, we find that

$$
H_{0}^{t} \sim L_{0}^{t}\left(\theta_{t}\right) \frac{\mathrm{e}^{\mathrm{i} k w r_{t}}}{\sqrt{k r_{t}}}
$$

as $R_{t} \rightarrow \infty$, where

$$
L_{0}^{t}\left(\theta_{t}\right)=-\frac{\sin \chi \mathrm{e}^{-\pi \mathrm{i} / 4} \sin \theta_{t} / 2}{\sqrt{w \pi} \sqrt{1-\cos \chi}\left(\cos \chi-\cos \theta_{t}\right)}+H_{0}^{t, p},
$$

where $H_{0}^{t, p}$ is a pole contribution given by

$$
H_{0}^{t, p}=\operatorname{sgn} \Psi_{t} \mathrm{e}^{\mathrm{i} w \cos \chi \Phi_{t}+\mathrm{i} w \sin \chi\left|\Psi_{t}\right|}
$$

when $-\chi<\theta_{t}<\chi$, and zero otherwise. The outer limit of this pole contribution matches to the remaining term (that explicitly contains $\theta_{t}$ ) in $h^{o}$. The equivalent uniformly-valid expansion for (1.4.2.10) is

$$
H_{0}^{t} \sim \frac{\mathrm{i} \sin \chi \mathrm{e}^{-\mathrm{i} \beta_{t}^{2}} \mathrm{e}^{\mathrm{i} k w r_{t}} \operatorname{sgn}\left(\Psi_{t}\right)}{2 \sqrt{1-\cos \chi} \sqrt{1+\cos \theta_{t}}} \operatorname{sgn}\left(\beta_{t}\right) \operatorname{erfc}\left[\mathrm{e}^{-\pi \mathrm{i} / 4}\left|\beta_{t}\right|\right]+H_{0}^{t, p}
$$

where

$$
\beta_{t}=\left(\cos \chi-\cos \theta_{t}\right) \sqrt{\frac{w R_{t}}{2 \sin ^{2} \theta_{t}}} .
$$

The pole contribution from $H_{0}^{t, p}$ matches to the outer solution, $h^{o}$, given by (1.3.0.1).

By combining (1.4.2.7) and (1.4.2.11), the overall scattered solution (i.e. without the pole term) for the trailing-edge inner region has outer limit

$$
\begin{aligned}
H_{t}^{t o t} & =H_{t}+H_{0}^{t} \mathrm{e}^{\mathrm{i} k w \cos \chi\left(2 \pm \Gamma / 2+\alpha_{t}\right)+\mathrm{i} k w \epsilon \sigma_{1}^{t}(0,0)} \\
& \sim\left(T\left(\theta_{t}\right)+\sqrt{k} L_{0}^{t}\left(\theta_{t}\right) \mathrm{e}^{\mathrm{i} k w \cos \chi\left(2 \pm \Gamma / 2+\alpha_{t}\right)+\mathrm{i} k w \epsilon \sigma_{1}^{t}(0,0)}\right) \frac{\mathrm{e}^{\mathrm{i} k w r_{t}}}{k \sqrt{r_{t}}} .
\end{aligned}
$$

This is matched onto the outer trailing-edge ray field (1.4.1.5) using Van Dyke's matching rule, yielding the final results;

$$
K_{t}\left(\theta_{t}\right)=T\left(\theta_{t}\right)+\sqrt{k} L_{0}^{t}\left(\theta_{t}\right) \mathrm{e}^{\mathrm{i} k w \cos \chi\left(2 \pm \Gamma / 2+\alpha_{t}\right)+\mathrm{i} k w \epsilon \sigma_{1}^{t}(0,0)} \quad \text { and } \quad g_{2}\left(\theta_{t}\right)=0 .
$$




\subsubsection{Trailing-Edge Transition Solution}

We now construct the trailing-edge transition solution $h_{t s}$, which cancels the pressure jump across the entire wake caused by the leading-edge field. We again split the solution into thickness-dependent, $h_{t s}^{(t)}$, and camber-dependent parts, $h_{t s}^{(c)}$, where the thicknessdependent part of the transition solution remains similar to the gust case of Tsai (1992), save for some rescaling, so

$$
h_{t s}^{(t)}=\frac{1}{\sqrt{k}} \mathcal{G}_{t}\left(\phi_{t}, \eta_{t}\right) \mathrm{e}^{\mathrm{i} k w \sigma_{t}\left(\phi_{t}, 0\right)}, \quad \eta_{t}=\sqrt{k} \psi_{t},
$$

with

$$
\mathcal{G}_{t}\left(\phi_{t}, \eta_{t}\right)=\frac{\mathrm{e}^{\mathrm{i} C_{ \pm}} \sqrt{w} \mathrm{e}^{\mathrm{i} \pi / 4}}{2 \sqrt{2}(\delta-w) \sqrt{\pi}} \int_{0}^{\phi_{t}} \frac{\eta_{t} \Delta p^{(t)}(\xi)}{\left(\phi_{t}-\xi\right)^{3 / 2}} \mathrm{e}^{\mathrm{i} w \eta_{t}^{2} / 2\left(\phi_{t}-\xi\right)} d \xi
$$

We can use the solution from Tsai (1992) because it depends only on the pressure jump at the trailing edge rather than explicitly on the nature of the incident perturbation. Similarly, the transition solution for the camber dependence is similar to that presented in Myers \& Kerschen (1997), subject to a rescaling in $k$, and a slight alteration of the phase to account for thickness dependence there. We take this solution in its final form

$$
h_{t s}^{(c)}=\frac{\mathrm{e}^{\mathrm{i} k w \phi_{t}}}{\sqrt{k}}\left[J_{t 0}\left(\phi_{t}, \eta_{t}\right)+\sqrt{k}\left(J_{t 1, a}\left(\phi_{t}, \eta_{t}\right)+J_{t 1, b}\left(\phi_{t}, \eta_{t}\right)\right)+O(\epsilon)\right]
$$

where the $J_{t i, j}$ are given by

$$
\begin{aligned}
J_{t 0}= & \frac{-\operatorname{sgn}(\psi) \mathrm{e}^{\mathrm{i} C_{ \pm}}}{2 \sqrt{2+\phi_{t}}} \mathrm{e}^{\mathrm{i} w \eta_{t}^{2} / 2\left(2+\phi_{t}\right)} \mathrm{e}^{\mathrm{i} k w \epsilon \sigma_{1}(0,0)}\left(P_{l}(0) \mathrm{e}^{\mathrm{i} k\left(\left(w-\delta M_{\infty}^{2}\right) \Gamma / 2+\epsilon w V(0) \int_{0}^{2} q\left(r_{t}, 0\right) d r\right)}\right. \\
& \left.-P_{l}(2 \pi) \mathrm{e}^{-\mathrm{i} k\left(\left(w-\delta M_{\infty}^{2}\right) \Gamma / 2+\epsilon w V(0) \int_{0}^{2} q\left(r_{t}, 2 \pi\right) d r\right)}\right) \operatorname{erfc}\left[\frac{\mathrm{e}^{-\pi \mathrm{i} / 4} \sqrt{w}\left|\eta_{t}\right|}{\sqrt{\phi_{t}\left(2+\phi_{t}\right)}}\right] \mathrm{e}^{\mathrm{i} k\left(w-\delta M_{\infty}^{2}\right)\left(2+\alpha_{t}\right)}
\end{aligned}
$$

$$
J_{t 1, a}=\mathrm{i} \epsilon w V(0) \eta J_{t 0} \int_{0}^{\phi_{t}} \frac{\partial q_{t}^{(c)}}{\partial \psi_{t}}\left(\phi_{t}^{\prime}, 0\right) d \phi_{t}^{\prime}-V(0) \epsilon \frac{\partial J_{t 0}}{\partial \eta} \int_{0}^{\phi_{t}} \int_{0}^{\phi_{t}^{\prime}} \frac{\partial q_{t}^{(c)}}{\partial \psi_{t}}\left(\phi_{t}^{\prime \prime}, 0\right) d \phi_{t}^{\prime \prime}
$$

and

$$
\begin{aligned}
J_{t 1, b}=- & \epsilon \frac{\mathrm{e}^{\mathrm{i} C_{ \pm}} w V(0) \mathrm{i}}{4 \pi \beta_{\infty}} \mathrm{e}^{\mathrm{i} k\left(w-\delta M_{\infty}^{2}\right)\left(2+\alpha_{t}\right)+\mathrm{i} k w \epsilon \sigma_{1}(0,0)}\left(P_{l}(0) \mathrm{e}^{\mathrm{i} k\left(\left(w-\delta M_{\infty}^{2}\right) \Gamma / 2+\epsilon w V(0) \int_{0}^{2} q\left(r_{t}, 0\right) d r\right)}\right. \\
& \left.+P_{l}(2 \pi) \mathrm{e}^{-\mathrm{i} k\left(\left(w-\delta M_{\infty}^{2}\right) \Gamma / 2+\epsilon w V(0) \int_{0}^{2} q\left(r_{t}, 2 \pi\right) d r\right)}\right) \int_{0}^{\phi_{t}} \frac{\eta_{t} b_{t}(\nu) \mathrm{e}^{\mathrm{i} w \eta_{t}^{2} / 2\left(\phi_{t}-\nu\right)}}{\left(\phi_{t}-\nu\right)^{3 / 2}} d \nu,
\end{aligned}
$$


with

$$
b_{t}\left(\phi_{t}\right)=\frac{2}{2+\phi_{t}} \int_{0}^{2} \sqrt{2+\phi_{t}-\xi} \sqrt{\xi} y^{(c) \prime \prime}(\xi) d \xi .
$$

Here, $J_{t 1, a}\left(\phi_{t}, \eta_{t}\right)$ is the correction in the trailing-edge transition region that cancels the discontinuity in the vertical velocity across the wake (and has zero pressure jump) caused by the leading-edge camber-related field, and $J_{t 1, b}\left(\phi_{t}, \eta_{t}\right)$ has continuous vertical velocity across the wake, but cancels the discontinuity in the pressure caused by the leading-edge camber-related field. For more details see Myers \& Kerschen (1997).

\subsubsection{Matching the Trailing-Edge Transition and Inner Solutions}

Here we match the trailing-edge transition and inner solutions to ensure that our final region completes our solution for the acoustic field anywhere in the solution domain. We note first that when we expand $H_{0}^{t}\left(\theta_{t}\right)$ from (1.4.2.11) in terms of transition variables it becomes $O\left(k^{-1}\right)$ and hence is negligible in the matching. Setting $\xi_{1}=\left|\eta_{t}\right| / \sqrt{\phi_{t}-\xi}$ in (1.4.3.1b) as done by Tsai (1992) gives

$$
G_{t}\left(\phi_{t}, \eta_{t}\right)=\frac{\operatorname{sgn}\left(\psi_{t}\right) \mathrm{e}^{\mathrm{i} C_{ \pm}} \sqrt{w} \mathrm{e}^{\mathrm{i} \pi / 4}}{\sqrt{2}(\delta-w) \sqrt{\pi}} \int_{\left|\eta_{t}\right| / \sqrt{\phi_{t}}}^{\infty} \Delta p^{(t)}\left(\phi_{t}-\eta_{t}^{2} / \xi_{i}^{2}\right) \mathrm{e}^{\mathrm{i} w \xi_{1}^{2} / 2} d \xi_{1} .
$$

We separate $\Delta p^{(t)}\left(\phi_{t}\right)$ into a term proportional to $\Delta p^{(t)}(0)$, and a correction of $O\left(t k^{-1 / 2}\right)$. This is given explicitly in Appendix C.

When we expand $G_{t}$ in terms of the variables $\Phi_{t}=k \phi_{t}$ and $\rho_{t}=\left|\eta_{t}\right| / \sqrt{\phi_{t}}=\left|\Psi_{t}\right| / \sqrt{\Phi_{t}}$ we see immediately that the second term in $\Delta p^{(t)}$ vanishes to $O\left(\epsilon k^{-1 / 2}\right)$, so

$$
h_{t s}^{(t)} \sim \frac{\operatorname{sgn}\left(\psi_{t}\right) \mathrm{e}^{\mathrm{i} k w \sigma_{t}\left(\phi_{t}, 0\right)}}{\sqrt{k}} \frac{\mathrm{e}^{\mathrm{i} C_{ \pm}} \mathrm{i} \Delta p^{(t)}(0)}{2(\delta-w)} \operatorname{erfc}\left(\mathrm{e}^{-\mathrm{i} \pi / 4} \rho_{t} \frac{\sqrt{w}}{\sqrt{2}}\right) .
$$

Writing (1.4.3.2) also in terms of these variables yields

$$
h_{t s}^{(c)} \sim \frac{\operatorname{sgn}\left(\psi_{t}\right) \mathrm{e}^{\mathrm{i} k w \sigma_{t}\left(\phi_{t}, 0\right)}}{\sqrt{k}} \frac{\mathrm{e}^{\mathrm{i} C_{ \pm}} \mathrm{i} \Delta p^{(c)}(0)}{2(\delta-w)} \operatorname{erfc}\left(\mathrm{e}^{-\mathrm{i} \pi / 4} \rho_{t} \frac{\sqrt{w}}{\sqrt{2}}\right) .
$$

The contributions from $J_{t 1}$ are negligible during the matching process. Recall the inner solution, (1.4.2.7), and note that for the transition region we require the expansion of $T$ that is uniformly valid for all $\theta_{t}$, i.e. $T^{u}\left(\theta_{t}\right)$. Expanding this in terms of the transition region variables, $\eta_{t}$ and $\phi_{t}$, and taking the limit of large $k$ we obtain

$$
H_{t} \sim \frac{\operatorname{sgn}\left(\psi_{t}\right) \mathrm{i} \Delta p(0) \mathrm{e}^{\mathrm{i} C_{ \pm}}}{2(\delta-w) \sqrt{k}} \operatorname{erfc}\left(\mathrm{e}^{-\mathrm{i} \pi / 4} \frac{\sqrt{w \eta_{t}^{2}}}{\sqrt{2 \phi_{t}}}\right) \mathrm{e}^{-\mathrm{i} w \eta_{t}^{2} / 2 \phi_{t}} \mathrm{e}^{\mathrm{i} k w r_{t}}
$$


where we use the approximation $\theta_{t} \sim \eta_{t} /\left(\sqrt{k} \phi_{t}\right)$. Finally, we note that in the trailingedge transition region $r_{t} \sim \phi_{t}+\eta_{t}^{2} /\left(2 k \phi_{t}\right)$, hence we see that these two scattered solutions, $h_{t s}$ and $H_{t}$, match since $\Delta p(0)=\Delta p^{(t)}(0)+\Delta p^{(c)}(0)$. We are not considering the $h_{l s}$ term in the matching here because it is not a field scattered from the trailing edge, and has already matched its corresponding scattered field from the leading edge.

\subsubsection{Far-Field Solution from the Trailing Edge}

The outer trailing-edge solution is given by (1.4.1.5) and (1.4.2.14). Following the work of Myers \& Kerschen (1997) and Tsai (1992), we expand this in terms of transition region variables, and expand the transition solution in terms of outer variables. When we write both in terms of transition region variables we see common terms for both camber and thickness. This ensures there is a matching between the trailing-edge transition and outer solutions, but we must subtract this common term from the total far-field solution to create a composite trailing-edge solution made up of the outer and transition solutions. The common term corresponding to the thickness parameter is

$$
h_{t}^{(t) c}=-\frac{\operatorname{sgn}\left(\psi_{t}\right) \mathrm{e}^{\mathrm{i} \pi / 4} \Delta p^{(t)}(0) \mathrm{e}^{\mathrm{i} C_{ \pm}} \sqrt{\phi_{t}}}{\sqrt{2 w \pi}\left|\eta_{t}\right| \sqrt{k}(\delta-w)} \mathrm{e}^{\mathrm{i} k w \phi_{t}+\mathrm{i} w \eta_{t}^{2} / 2 \phi_{t}+\mathrm{i} k \epsilon w \sigma_{t_{1}}\left(r_{t}, 0\right)},
$$

while for the camber parameters the common term is

$$
\begin{array}{r}
h_{t}^{(c) c}=-\frac{\operatorname{sgn}\left(\psi_{t}\right) \mathrm{e}^{\pi \mathrm{i} / 4} \mathrm{e}^{\mathrm{i} C_{ \pm}} \sqrt{\phi_{t}}}{2 \sqrt{\pi w} \sqrt{k}\left|\eta_{t}\right|} \mathrm{e}^{\mathrm{i} k w \phi_{t}+\mathrm{i} w \eta_{t}^{2} / 2 \phi_{t}}\left[1+\frac{\mathrm{i} \sqrt{k} \eta_{t} \epsilon w V(0)}{\phi_{t}} \int_{0}^{\phi_{t}} \phi_{t}^{\prime} \frac{\partial q^{(c)}}{\partial \psi_{t}}\left(\phi_{t}^{\prime}, 0\right) d \phi_{t}^{\prime}\right] \\
\left(P_{l}(0) \mathrm{e}^{\mathrm{i} k\left(\left(w-\delta M_{\infty}^{2}\right) \Gamma / 2+\epsilon w V(0) \int_{0}^{2} q(r, 0) d r\right)}-P_{l}(2 \pi) \mathrm{e}^{-\mathrm{i} k\left(\left(w-\delta M_{\infty}^{2}\right) \Gamma / 2+\epsilon w V(0) \int_{0}^{2} q(r, 2 \pi) d r\right)}\right) \\
\mathrm{e}^{\mathrm{i} k\left(w-\delta M_{\infty}^{2}\right)\left(2+\alpha_{t}\right)+\mathrm{i} k w \epsilon \sigma_{1}^{t}(0,0)} .
\end{array}
$$

To obtain a uniformly-valid composite solution in the outer region of the trailing edge, denoted by $h_{t}^{u}$, we must subtract this common term, therefore giving

$$
h_{t}^{u}=h_{t}+h_{t s}-h_{t}^{c}
$$

We did not have to subtract a common term when considering the leading-edge transition solution because the leading-edge transition solution had a negligible effect in the far field (except at $O(\sqrt{k})$ angles from $\theta=0$ which we corrected with the trailing-edge transition solution). 
The final expression for total far-field trailing-edge acoustic potential is given by $h_{t}^{u}\left(r_{t}, \theta_{t}\right) \sim \frac{\mathrm{e}^{\mathrm{i} C_{ \pm}} \mathrm{e}^{\mathrm{i} k w \sigma_{t}\left(r_{t}, \theta_{t}\right)}}{\sqrt{2} k \sqrt{r_{t}}(\delta-w \cos \theta)}\left\{-\operatorname{sgn}\left(\psi_{t}\right) \mathrm{ie}^{-2 \mathrm{i} k w\left(1-\cos \theta_{t}\right)}\left(P_{+}-P_{-}\right)\right.$ $\operatorname{erfc}\left[\mathrm{e}^{-\pi \mathrm{i} / 4} \sqrt{2 k w(1-\cos \theta)}\right]-\frac{2 \sqrt{k w} V(0)\left(\alpha_{i}-\epsilon y^{(c) \prime}(2)\right)\left(P_{+}-P_{-}\right) \mathrm{e}^{\pi \mathrm{i} / 4}}{\sqrt{\pi} \beta_{\infty}}$ $\left(1-\sqrt{2 k w \pi(1-\cos \theta)} \mathrm{e}^{-2 \mathrm{i} k w(1-\cos \theta)-\pi \mathrm{i} / 4} \operatorname{erfc}\left[\mathrm{e}^{-\pi \mathrm{i} / 4} \sqrt{2 k w(1-\cos \theta)}\right]\right)$ $\left.+\frac{\operatorname{sgn}\left(\psi_{t}\right) \epsilon \sqrt{k w} V(0)\left(P_{+}+P_{-}\right)}{\sqrt{2} \pi \beta_{\infty}} \sqrt{k w(1-\cos \theta)} \int_{0}^{\infty} \mathrm{e}^{\mathrm{i} k w(1-\cos \theta) \xi} b_{t}(\xi) d \xi\right\}$ $+\frac{\operatorname{sgn}(\psi) \mathrm{e}^{\mathrm{i} C_{ \pm}} \mathrm{e}^{\mathrm{i} k w \sigma_{t}\left(r_{t}, \theta_{t}\right)}}{k \sqrt{r_{t}}(\delta-w)}\left\{\frac{\Delta p^{(t)}(0) \mathrm{e}^{\pi \mathrm{i} / 4}\left|\sin \theta_{t} / 2\right|}{\sqrt{2 \pi w}(\delta-w \cos \theta)}\right.$

$+\frac{\Delta p^{(t)}(0)}{\sqrt{2}} \mathrm{i} \sqrt{k} \mathrm{e}^{-2 \mathrm{i} k w\left(1-\cos \theta_{t}\right)} \operatorname{erfc}\left[\mathrm{e}^{-\pi \mathrm{i} / 4} \sqrt{2 k w(1-\cos \theta)}\right]$ $+\frac{t k \mathrm{i} \beta_{\infty} \sin \chi \mathrm{e}^{\pi \mathrm{i} / 4} \sqrt{w}(\delta-w)}{2 \pi^{3 / 2} \sqrt{\cos \chi+1}(\cos \chi-1)}\left(1-\frac{(\gamma+1) M_{\infty}^{4}}{2 \beta_{\infty}^{4} w^{2}}(\delta-w)^{2}\right) \mathrm{e}^{\mathrm{i} k\left(w-\delta M_{\infty}^{2}\right)\left(2+\alpha_{t}\right)+\mathrm{i} k w \epsilon \sigma_{1}(0,0)}$ $\left[\mathrm{e}^{\mathrm{i} k\left(w-\delta M_{\infty}^{2}\right) \Gamma / 2+\mathrm{i} k w \epsilon \sigma_{l_{1}}(2,0)}\left\{\mathrm{e}^{\pi \mathrm{i} / 4} \frac{\sqrt{\pi}}{\sqrt{2 w}} \int_{0}^{2}\left(\frac{a}{\tau}+\frac{\beta_{\infty}}{t^{\prime} \tau^{3 / 2}} \int_{0}^{\tau} \frac{\partial q^{(t)}}{\partial \theta}(r, 0) d r\right)\right.\right.$ $\mathrm{e}^{\mathrm{i} k w(1-\cos \theta) \tau} \operatorname{erfc}\left[\mathrm{e}^{-\pi \mathrm{i} / 4} \sqrt{2-\tau} \sqrt{k w(1-\cos \theta)}\right] d \tau+2 a \sqrt{k(1-\cos \theta)}$ $\int_{2}^{\infty} \mathrm{e}^{\mathrm{i} k w(1-\cos \theta) \xi^{2} / 2}\left[\log [2]-\log \left[\xi^{2} / 2\right]-\log \left[1-\sqrt{1-4 / \xi^{2}}\right]\right] d \xi$ $+\frac{\beta_{\infty}}{t^{\prime}} \int_{0}^{2} \frac{\partial q^{(t)}}{\partial \theta}(r, 0) d r \sqrt{2 k(1-\cos \theta)} \int_{2}^{\infty} \sqrt{1-4 / \xi^{2}} \mathrm{e}^{\mathrm{i} k w(1-\cos \theta) \xi^{2} / 2} d \xi-$ $\left.\frac{\sqrt{\pi}}{\sqrt{w}} \operatorname{erfc}\left[\mathrm{e}^{-\pi \mathrm{i} / 4} \sqrt{2 k w(1-\cos \theta)}\right] \int_{0}^{2}\left(\frac{a}{\tau}+\frac{\beta_{\infty}}{t^{\prime} \tau^{3 / 2}} \int_{0}^{\tau} \frac{\partial q^{(t)}}{\partial \theta}(r, 0) d r\right) \frac{d \tau}{\sqrt{2-\tau}}\right\}$ $+\mathrm{e}^{-\mathrm{i} k\left(w-\delta M_{\infty}^{2}\right) \Gamma / 2+\mathrm{i} k w \epsilon \sigma_{l_{1}}(2,2 \pi)}\left\{\mathrm{e}^{\pi \mathrm{i} / 4} \frac{\sqrt{\pi}}{\sqrt{2 w}} \int_{0}^{2}\left(\frac{a}{\tau}+\frac{\beta_{\infty}}{t^{\prime} \tau^{3 / 2}} \int_{0}^{\tau} \frac{\partial q^{(t)}}{\partial \theta}(r, 2 \pi) d r\right)\right.$ $\mathrm{e}^{\mathrm{i} k w(1-\cos \theta) \tau} \operatorname{erfc}\left[\mathrm{e}^{-\pi \mathrm{i} / 4} \sqrt{2-\tau} \sqrt{k w(1-\cos \theta)}\right] d \tau+2 a \sqrt{k(1-\cos \theta)}$ $\int_{2}^{\infty} \mathrm{e}^{\mathrm{i} k w(1-\cos \theta) \xi^{2} / 2}\left[\log [2]-\log \left[\xi^{2} / 2\right]-\log \left[1-\sqrt{1-4 / \xi^{2}}\right]\right] d \xi$ $+\frac{\beta_{\infty}}{t^{\prime}} \int_{0}^{2} \frac{\partial q^{(t)}}{\partial \theta}(r, 2 \pi) d r \sqrt{2 k(1-\cos \theta)} \int_{2}^{\infty} \sqrt{1-4 / \xi^{2}} \mathrm{e}^{\mathrm{i} k w(1-\cos \theta) \xi^{2} / 2} d \xi-$ $\left.\left.\frac{\sqrt{\pi}}{\sqrt{w}} \operatorname{erfc}\left[\mathrm{e}^{-\pi \mathrm{i} / 4} \sqrt{2 k w(1-\cos \theta)}\right] \int_{0}^{2}\left(\frac{a}{\tau}+\frac{\beta_{\infty}}{t^{\prime} \tau^{3 / 2}} \int_{0}^{\tau} \frac{\partial q^{(t)}}{\partial \theta}(r, 2 \pi) d r\right) \frac{d \tau}{\sqrt{2-\tau}}\right\}\right]$ $\left.+\frac{\mathrm{i} \sin \chi \mathrm{e}^{-\mathrm{i} \beta_{t}^{2}}(\delta-w) k \sqrt{r_{t}}}{2 \sqrt{1-\cos \chi} \sqrt{1+\cos \theta_{t}}} \operatorname{sgn}\left(\beta_{t}\right) \operatorname{erfc}\left[\mathrm{e}^{-\pi \mathrm{i} / 4}\left|\beta_{t}\right|\right] \mathrm{e}^{\mathrm{i} k\left(w \cos \chi-\delta M_{\infty}^{2}\right)\left(2 \pm \Gamma / 2+\alpha_{t}\right)} \mathrm{e}^{\mathrm{i} k w \epsilon \sigma_{1}^{t}(0,0)}\right\}$, 
where

$$
P_{ \pm}=\frac{\mathrm{i}}{\sqrt{2}}(w-\delta) P_{l}(0 \pm) \mathrm{e}^{ \pm \mathrm{i} k\left(\left(w-\delta M_{\infty}^{2}\right) \Gamma / 2+w \epsilon V(0) \int_{0}^{2} q(r, 0 \pm) d r\right)+\mathrm{i} k\left(w-\delta M_{\infty}^{2}\right)\left(2+\alpha_{t}\right)+\mathrm{i} k w \epsilon \sigma_{1}(0,0)}
$$

and the camber-dependent pressure jump at the trailing edge is given by $P_{+}-P_{-}$.

\subsection{Total Far-Field Solution}

At this point we briefly summarise our analysis. The total far-field scattered pressure directivity is dominated by direct scattering from the leading and trailing edges, and in addition more sound is generated by the interaction of the incident sound wave with the aerofoil and the locally non-uniform mean flow in the leading-edge region (Section 1.2). In the outer region the length scale of the mean-flow gradients scales with the chord length of the aerofoil, and the distortion of the sound wave is sufficiently slow that no additional sound is generated in this region. However, sound propagates away from the leadingand trailing-edge regions through the outer region, and undergoes a refractive phase shift of $O(1)$ (Section 1.3.1). There are transition regions above and below the aerofoil which account for the surface curvature, and are seen as rays propagating from the leading edge along the upper and lower surfaces of the aerofoil (Section 1.3.2). These rays are then scattered by the trailing edge (Sections 1.4 .1 and 1.4.2). The trailing-edge transition solution cancels the discontinuous pressure across the wake caused by the leading-edge ray fields above and below the aerofoil (Section 1.4.3). A further contribution to the outer solution is $h^{o}$ from (1.3.0.1), which arises from the incident sound wave being directly reflected or blocked by the aerofoil surface. In the far-field this only has an effect in a small $O(1 / r)$ region at $\theta=\chi$, and at distances of $r=O(k)$ from the aerofoil this direct field is overpowered by the scattered Fresnel fields in a similar manner to that discussed in Peake \& Kerschen (2004), therefore we do not consider its effects from now on and instead discuss just the scattered solution.

We have obtained solutions for the leading- and trailing-edge ray fields, $h_{l}^{u}$ and $h_{t}^{u}$. To obtain a uniformly-valid expansion, we replace any non-uniform terms with their uniformly-valid counterparts, as explained in Section 1.3.1. We add the two fields to find the total acoustic far-field potential,

$$
h=h_{l}^{u}+h_{t}^{u},
$$


which can be written as

$$
h=\mathrm{e}^{\mathrm{i} k w \sigma_{l}}\left[D_{l}^{u}(r, \theta)-D_{t}^{u}(r, \theta) \mathrm{e}^{\mathrm{i} k w \sigma_{s}}\right]
$$

where $\sigma_{s}$ is the phase shift between the leading- and trailing-edge ray fields, as measured by an observer in the far field at angle $\theta$ from the leading edge. The functions, $D_{l, t}^{u}(r, \theta)$, are the uniformly-valid contributions to the total pressure from the leading and trailing edge respectively. We choose a negative sign between the leading- and trailing-edge fields so that the real parts of the Fresnel region contributions both have the same sign.

In the far field, the phase shift, $\sigma_{s}$, is given by

$$
\begin{aligned}
\sigma_{s}^{ \pm} & =\sigma_{t}-\sigma_{l} \\
& =\frac{V(\theta)}{\beta_{\infty}}\left(2 \alpha_{i} \sin \theta+\cos \theta\left( \pm \alpha_{g} \pi+\beta_{\infty} \alpha_{t}\right)\right)+\left(2 \pm \frac{\alpha_{g} \pi}{\beta_{\infty}}+\alpha_{t}\right) \cos \theta-\mathrm{i} k w \epsilon \sigma_{1}(0,0),
\end{aligned}
$$

and $\alpha_{g}$ is as defined in (1.4.1.3). The " \pm " denotes the phase shift above and below the aerofoil respectively, and is present due to the non-zero mean circulation. The first term in (1.5.0.2) corresponds to the different refraction experienced by the sound emanating from the leading- and trailing-edge sources en-route to an observer above or below the aerofoil, whilst the second term arises simply from the fact that the leading and trailing edges are located at different positions in $(\phi, \psi)$ space. The final term is due to the distortion of the incident sound wave as it approaches the leading edge, and is a constant. The term $\alpha_{g}$ is crucial in explaining why the acoustic pressures above and below the aerofoil differ as we alter the camber, thickness and angle of attack. We return to this in the next section.

\subsection{Results and Discussion}

\subsubsection{Far-Field Pressure Directivity for the Scattered Sound}

Here we present results for the far-field scattered pressure directivity (i.e. not including the incident wave), as determined from our analytic solutions $h_{l}^{u}$ and $h_{t}^{u}$. The pressure directivity is plotted in physical space, and polar coordinates $\left(r_{p}, \theta_{p}\right)$ in physical space 
are related to $(r, \theta)$ in potential-streamfunction space via

$$
\begin{aligned}
r & =\left(1-M_{\infty}^{2} \sin \theta\right)^{1 / 2} r_{p}+O\left(t, \alpha_{\mathrm{eff}}\right), \\
\cos \theta & =\frac{\beta_{\infty} \cos \theta_{p}}{\sqrt{1-M_{\infty}^{2} \cos ^{2} \theta_{p}}} .
\end{aligned}
$$

The $O\left(t, \alpha_{\text {eff }}\right)$ term in (1.6.1.1) provides only a phase shift to the total solution in the factor $\mathrm{e}^{\mathrm{i} k w \sigma_{l}}$ from (1.5.0.1), and hence does not affect the amplitude of the pressure directivity (Myers \& Kerschen, 1997). Our uniformly-valid composite expansions are plotted for $r_{p}$ greater than the Rayleigh distance, $O(k)$, to ensure that the acoustic far-field behaviour is captured correctly (i.e. the Fresnel regions from the leading and trailing edges have overlapped). In what follows, we omit the subscripted $p$ and refer only to physical space coordinates, and set $k_{3}=0$ in (1.1.2.4b).

We explicitly consider the NACA 4-digit series of aerofoils to illustrate our results as these are well known and widely used in industry (Abbott \& Von Doenhoff, 1959). The surfaces of such aerofoils are given by the non-dimensionalised functions

$$
y_{ \pm}=Y^{(c)} \pm y^{(t)} \cos \varphi, \quad x_{ \pm}=x \pm y^{(t)} \sin \varphi
$$

where \pm denotes the upper and lower surfaces of the aerofoil respectively, and $(x, y)$ are Cartesian coordinates with origin at the aerofoil leading edge. We define $Y^{(c)}(x), y^{(t)}(x)$ and $\varphi(x)$ by

$$
\begin{aligned}
Y^{(c)} & =-\alpha_{i}^{\prime} x+y^{(c)}(x), \\
\epsilon y^{(c)} & =\left\{\begin{array}{ll}
\frac{m x}{p^{2}}\left(2 p-\frac{x}{2}\right) & 0 \leq x \leq 2 p \\
\frac{m(2-x)}{(1-p)^{2}}\left(1+\frac{x}{2}-2 p\right) & 2 p \leq x \leq 2
\end{array},\right. \\
y^{(t)} & =\frac{2 t^{\prime}}{0.2}\left(a_{1} \sqrt{\frac{x}{2}}-a_{2} \frac{x}{2}-a_{3} \frac{x^{2}}{(2)^{2}}+a_{4} \frac{x^{3}}{(2)^{3}}-a_{5} \frac{x^{4}}{(2)^{4}}\right), \\
\varphi(x) & =\arctan \left[\frac{d y^{(c)}}{d x}+\alpha_{i}\right],
\end{aligned}
$$

with

$$
a_{1}=0.2969, \quad a_{2}=0.1260, \quad a_{3}=0.3516, \quad a_{4}=0.2843, \quad a_{5}=0.1036 \text {. }
$$

The standard choice of $a_{5}$ is 0.1015 (Abbott \& Von Doenhoff, 1959), however our choice of $a_{5}$ sets the position of the trailing edge to be $(2,0)$ which proves more convenient for computation. Analytically this small alteration is negligible to the orders retained 
during calculations. The superscripts $c$ and $t$ denote camber and thickness respectively, while the term $-\alpha_{i} x$ in (1.6.1.2) corresponds to the aerofoil being at the angle of attack $\alpha_{i}=\epsilon \alpha_{i}^{\prime}$. Here, 2 is the chord length, $0 \leq x \leq 2$ is the position along the chord, $\epsilon y$ is the half thickness at a given value of $x$ from the centreline to the surface, $t=\epsilon t^{\prime}$ is the maximum thickness as a fraction of the chord length, $m$ is the maximum camber as a fraction of the chord length, and $p$ is the location of the maximum camber along the centreline as a fraction of the chord length. A NACA 4-digit series aerofoil is defined entirely by the choice of $p, m$ and $t: 100 m$ gives the first digit, $10 p$ gives the second digit and $100 t$ gives the last two digits.

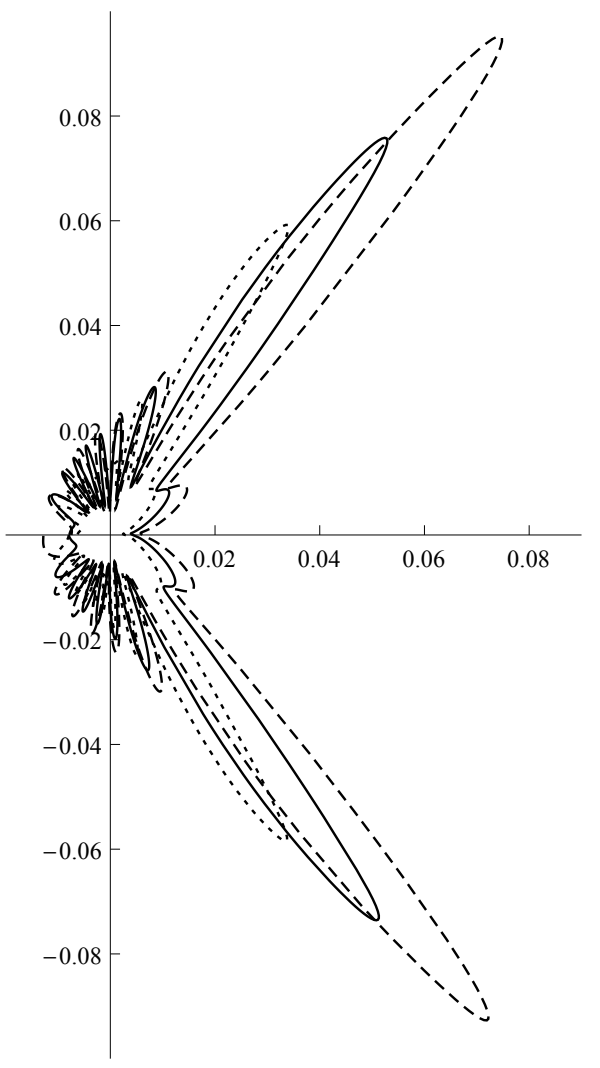

(a) Full range.

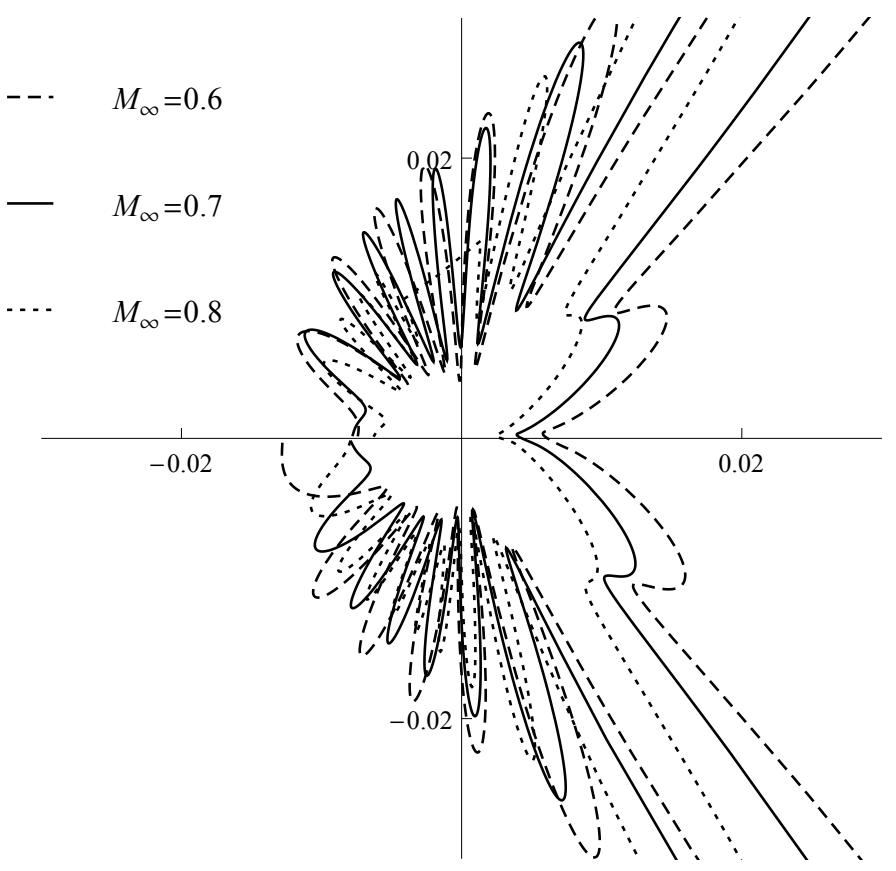

(b) Centre detail.

Figure 1.6: Far-field scattered pressure directivity for a NACA 1112 aerofoil, $k=6$, $\alpha_{i}=0^{\circ}, \chi=45^{\circ}, k w=10$. The Mach number is varied from 0.6 to 0.8 .

Figure 1.6 shows the effect of changing the freestream Mach number, $M_{\infty}$. An increase in Mach number increases the acoustic pressure amplitude everywhere around the aerofoil, and increases the modulation in the upstream direction (the left-half plane), i.e. a larger Mach number produces more lobes in the upstream direction. This effect arises from the relationship between $w$ and $M_{\infty}$ (see (1.1.2.4b)); as $M_{\infty}$ increases, so too does $w$, causing a more rapid phase variation. There is an extra modulation of the 


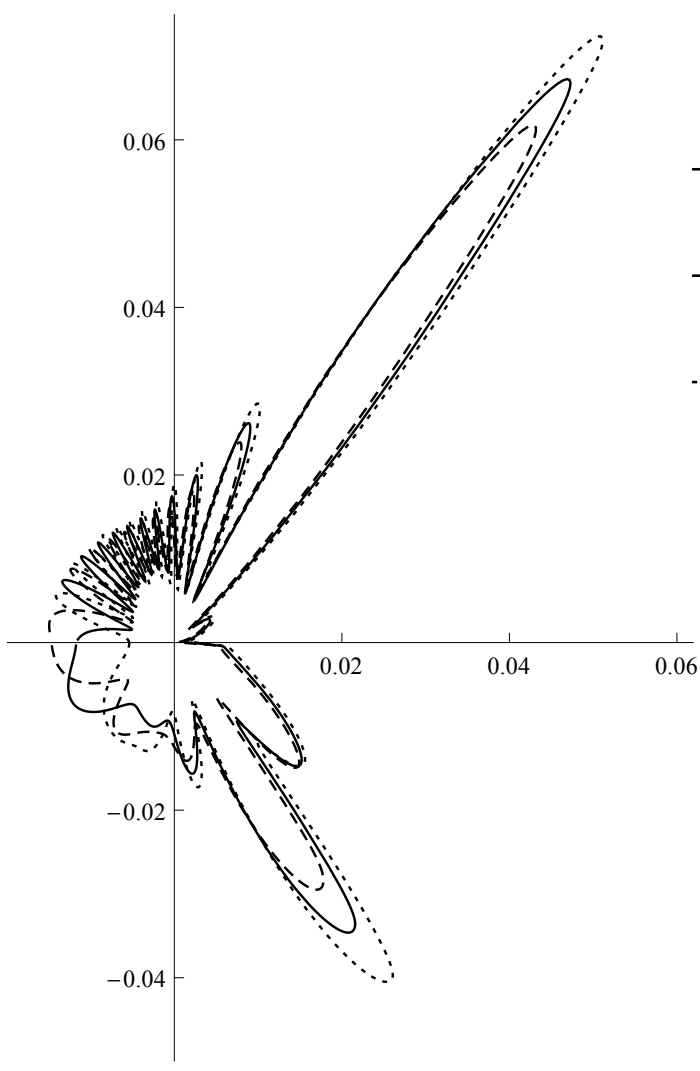

(a) Full range.

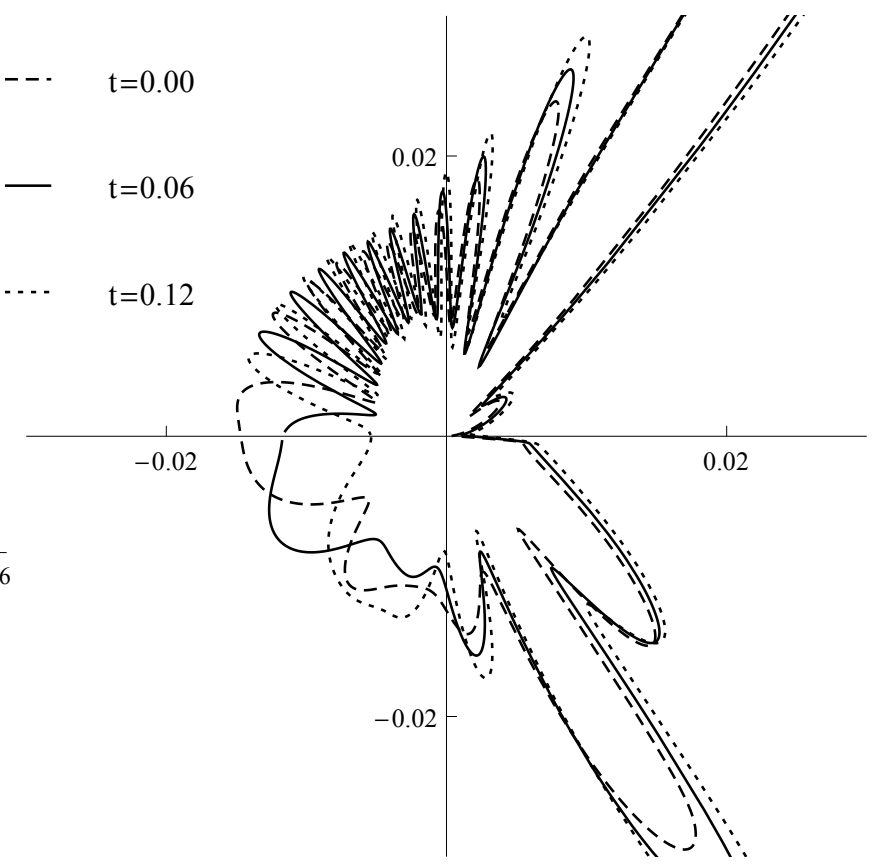

(b) Centre detail.

Figure 1.7: Far-field scattered pressure directivity for a NACA 4-digit aerofoil with 10\% maximum camber at $10 \%$ chord length, $k=6, \alpha_{i}=0^{\circ}, \chi=45^{\circ}, k w=10, M_{\infty}=0.7$. The thickness ratio is varied from $0 \%$ to $12 \%$.

phase above the aerofoil when compared to below, due to the phase shift between the leading and trailing edges; particularly the term depending on mean loading parameter $\alpha_{g} V(\theta)$. As analysed in Myers \& Kerschen (1997), this adds to the phase variation in the upper half plane, but subtracts from it in the lower half plane, due to the sign of the perturbation flow velocity $\epsilon q$; a positive $q$ (i.e. above the aerofoil) gives a larger speed of the surrounding flow in the downstream direction, whereas a negative sign decreases the speed, hence reducing the modulation. We see that the pressure directivity in the Fresnel regions (the large lobes above and below the aerofoil) is finite thanks to our uniformly-valid solution; this is a feature of all results given here.

The effect of varying aerofoil thickness is shown in Figure 1.7. As we increase thickness, we decrease the magnitude of the pressure levels directly upstream (the negative real axis). This is a result of the slowing of the steady flow as it approaches a thick body. The pressure in the Fresnel regions increases in magnitude, due to multiplicative factors of $t \sqrt{k}$ appearing in the corrections to the pressure amplitude that come from 


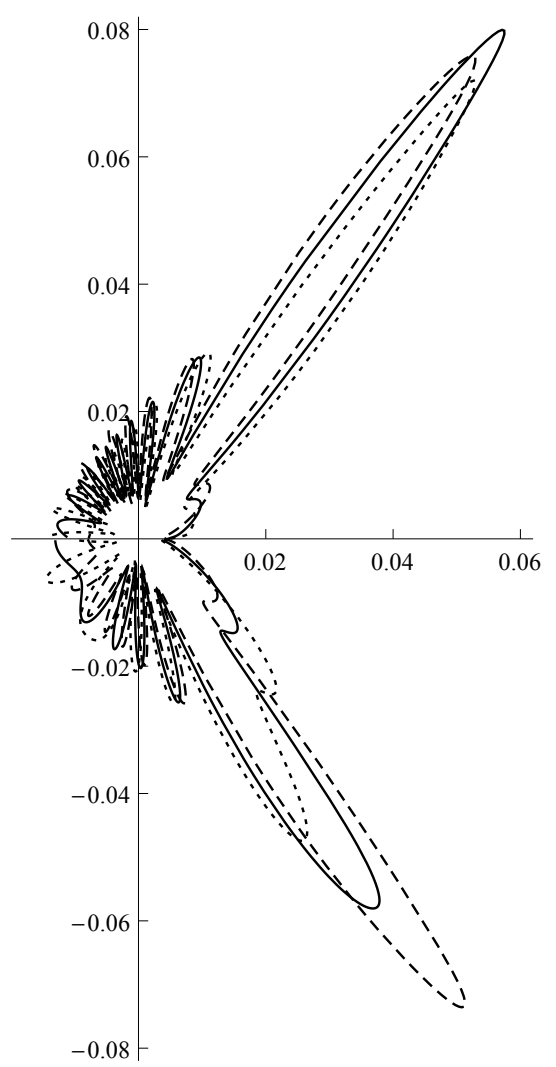

(a) Full range.

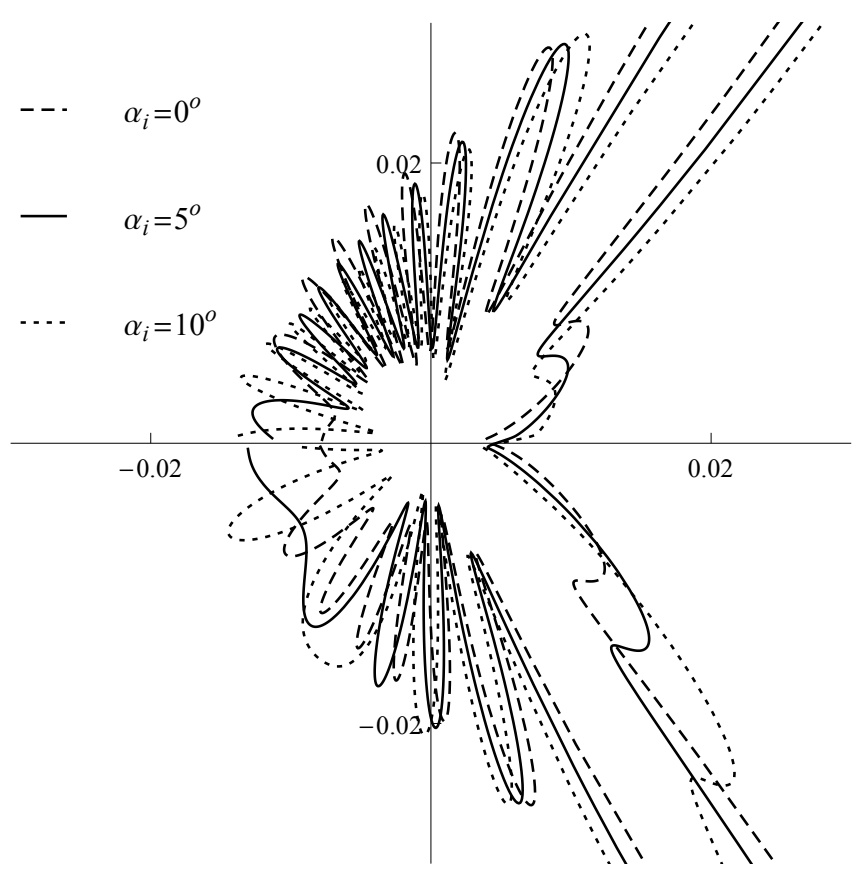

(b) Centre detail.

Figure 1.8: Far-field scattered pressure directivity for a NACA 1112 aerofoil with $k=6$, $\chi=45^{\circ}, k w=10, M_{\infty}=0.7$. The angle of attack is varied from $0^{\circ}$ to $10^{\circ}$.

(1.2.0.3). This effect is caused by additional Fresnel contributions being produced for a thick aerofoil hence we see increased reflection or shadow contributions.

Figure 1.8 shows the effect of varying the angle of attack of a NACA 1112 aerofoil. There is little amplification in the overall magnitude of the radiation (excluding in the Fresnel regions), due to the increase in $\alpha_{\text {eff }}$ being only small when we increase the angle of attack $\alpha_{i}$. Recall the significance of $\alpha_{\text {eff }}$ is that in the inner leading-edge region the camber-dependent part of $\epsilon q$ scales multiplicatively with $\alpha_{\text {eff }}$, thereby inducing the effect of both the angle of attack of the mean chord line and the camber relative to the mean chord line. The modulation is increased above the aerofoil for greater incidence angle, for similar reasons to those discussed for increasing Mach number in Figure 1.6. The pressure amplitude in the Fresnel regions varies in an apparently complicated way with varying $\alpha_{i}$, and this is caused by variations in magnitude of the directivity function, along with the phase shift between the leading and trailing Fresnel expansions being different above and below the aerofoil, which is discussed in more detail later.

Figure 1.9 shows the effect of varying $\chi$, the angle of propagation of the incident sound 


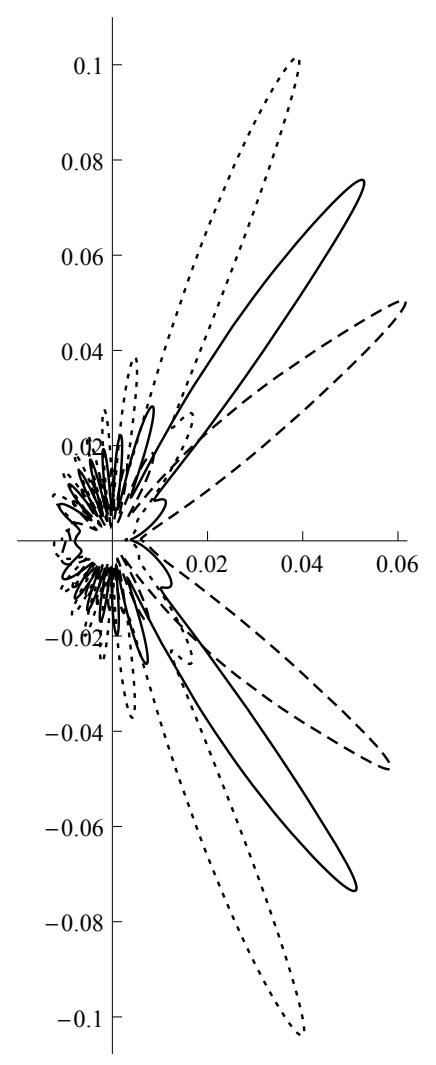

(a) Full range.

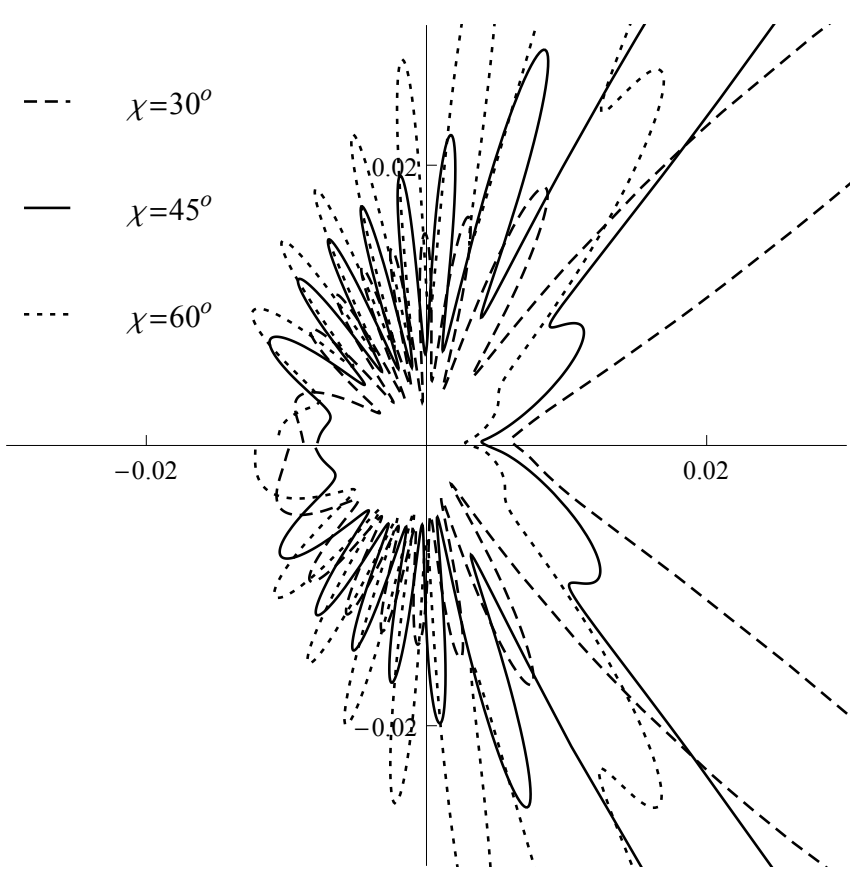

(b) Centre detail.

Figure 1.9: Far-field scattered pressure directivity for a NACA 1112 aerofoil with $k=6$, $\alpha_{i}=0^{\circ}, k w=10, M_{\infty}=0.7$. The angle of the incident sound wave, $\chi$, is varied from $30^{\circ}$ to $60^{\circ}$.

wave. Clearly as we alter $\chi$ the shadow and reflection boundaries move, and hence the directions of the Fresnel regions (which lie on these boundaries) also move. We also notice that there is an increase in the magnitude of the sound pressure level as we increase $\chi$. This is caused by the fact that the amplitude of the flat-plate solution, (1.2.1.9), scales as $\sin (\chi / 2)$, corresponding to the increase in the blocking of unsteady momentum by the aerofoil as the sound wave is rotated closer to the normal direction. Figure 1.10 shows the effect of varying $k w$. Physically this corresponds to varying the frequency of the incident sound wave. In the upstream direction, the pressure directivity increases in magnitude as $k w$ increases, since $w$ is a multiplicative factor of the pressure (recall, the pressure can be obtained from the modified potential $h$ via (1.1.2.5)). We have found that the flat-plate solution has $h \sim(k w)^{-1 / 2} \mathrm{e}^{\mathrm{i} k w r}$, hence the flat-plate pressure contribution has a multiplicative factor of $\left.w^{1 / 2}\right)$. The other effect of increasing $k w$ is simply to increase the modulation everywhere around the aerofoil (due to $k w$ being the scaling of all phase terms in the far-field sound). 


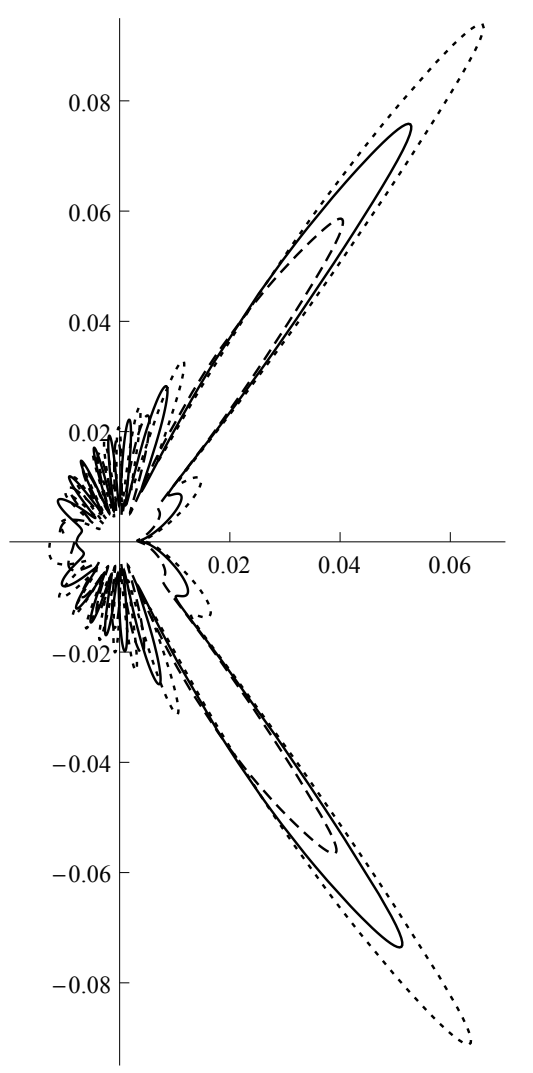

(a) Full range.

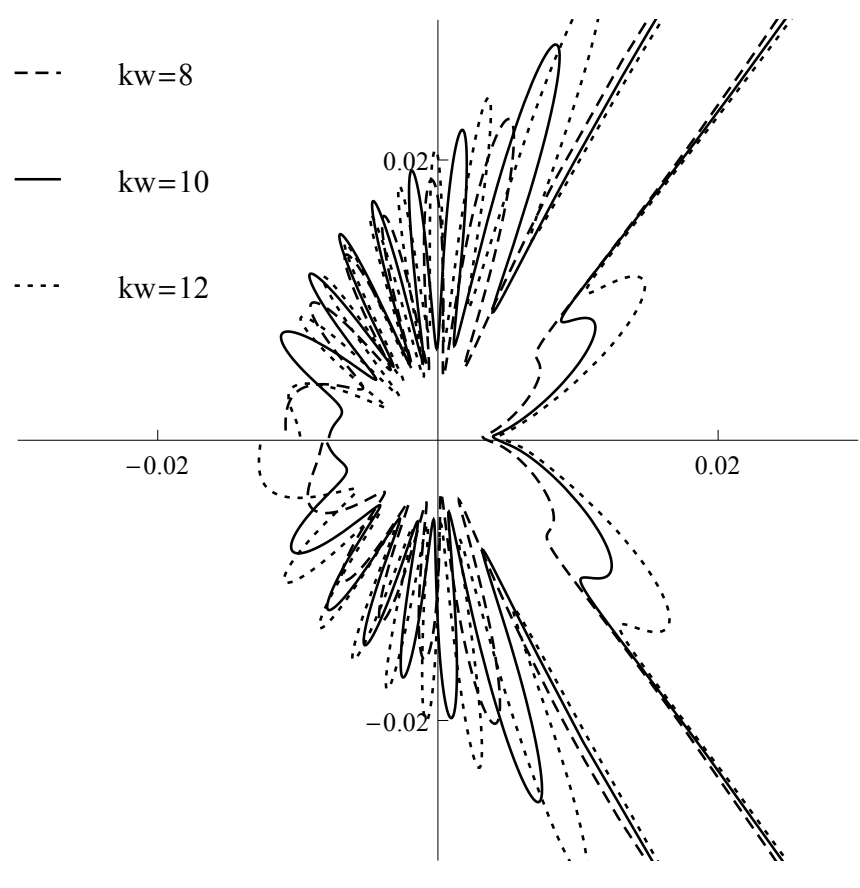

(b) Centre detail.

Figure 1.10: Far-field scattered pressure directivity for a NACA 1112 aerofoil with $k=6$, $\alpha_{i}=0^{\circ}, \chi=45^{\circ}, M_{\infty}=0.7$. The acoustic frequency, $k w$, is varied from 8 to 12 .

Finally, Figure 1.11 shows the effect of varying the percentage of maximum camber of the aerofoil for an incident sound wave emanating from far downstream i.e. from the bottom right of Figure 1.1. The modulation of the far-field pressure directivity is due to the interference of the leading- and trailing-edge fields, and as can be seen in the previous results is strongest in the upstream direction. This time, however, the Fresnel regions are directed upstream and thereby envelope much of the modulation, so that the directivity in Figure 1.11 is less oscillatory than for sound incident from upstream. The upper leading-edge Fresnel zone is visibly modulated however, and this modulation increases with increasing camber; this is for identical reasons as to the increasing modulation found in Figures 1.6 and 1.8. We also see here another example of a complicated variation in the magnitude of the pressure with varying aerofoil geometry in the Fresnel regions, similar to Figure 1.8. 


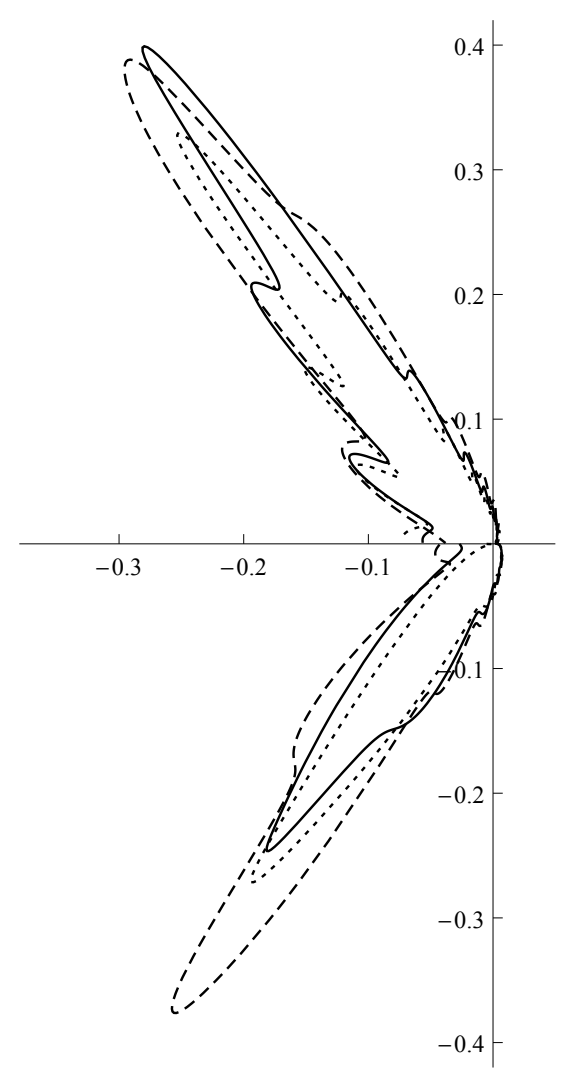

(a) Centre detail.

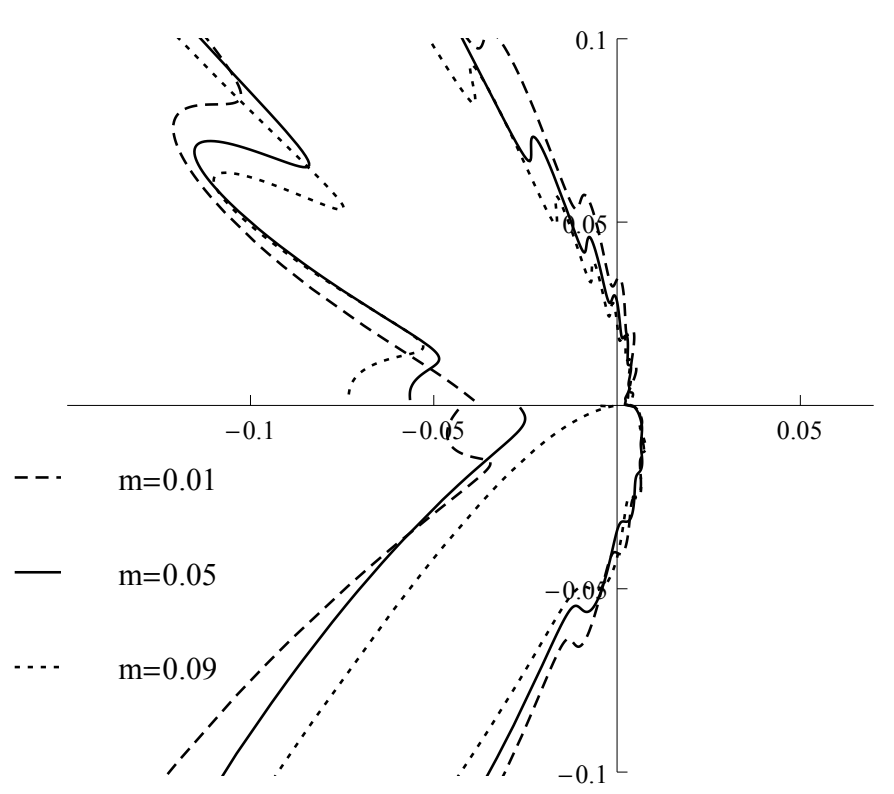

(b) Full range.

Figure 1.11: Far-field scattered pressure directivity for a NACA 4-digit aerofoil with maximum camber at $10 \%$ chord length, and $12 \%$ thickness, $k=6, k w=10, \alpha_{i}=0^{\circ}$, $\chi=135^{\circ}, M_{\infty}=0.7$. The percentage of maximum camber is varied from $1 \%$ to $9 \%$.

$\begin{array}{cccc}\alpha_{i} & k w \sigma_{s}^{+} & k w \sigma_{s}^{-} & k w\left(\sigma_{s}^{+}-\sigma_{s}^{-}\right) \\ -5^{\circ} & 0.46 & 2.69 & -2.23 \\ -3^{\circ} & 0.91 & 2.25 & -1.34 \\ 0^{\circ} & 1.58 & 1.58 & 0.00 \\ 3^{\circ} & 2.25 & 0.91 & 1.34 \\ 5^{\circ} & 2.69 & 0.46 & 2.23 \\ 7^{\circ} & 3.14 & 0.01 & 3.13 \\ 10^{\circ} & -2.47 & -0.66 & -1.81\end{array}$

Table 1.1: Values of the phase shift above and below the flat-plate aerofoil modulo $2 \pi$.

\subsubsection{Total Far-Field Pressure}

In what follows we set $M_{\infty}=0.7, k w=10$ and $\chi=45^{\circ}$, and investigate the maximum and minimum total scattered far-field pressure, by summing the incident pressure and the scattered pressure, and normalizing by the incident pressure. The maximum and 


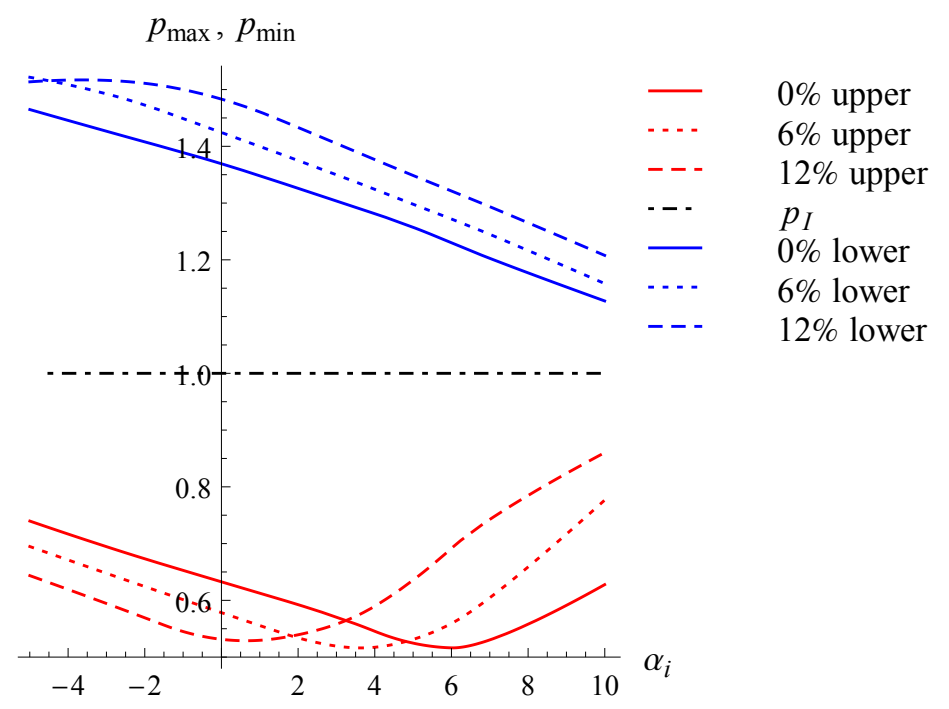

Figure 1.12: Maximum and minimum total far-field pressure (normalised with respect to the incident pressure), as functions of angle of attack, for uncambered NACA 4-digit aerofoils. The legend denotes maximum thickness in $\%$ of chord length and the location; either in the upper (shadow) or lower (reflection) Fresnel region. $p_{I}$ denotes the pressure generated by the incident sound wave.

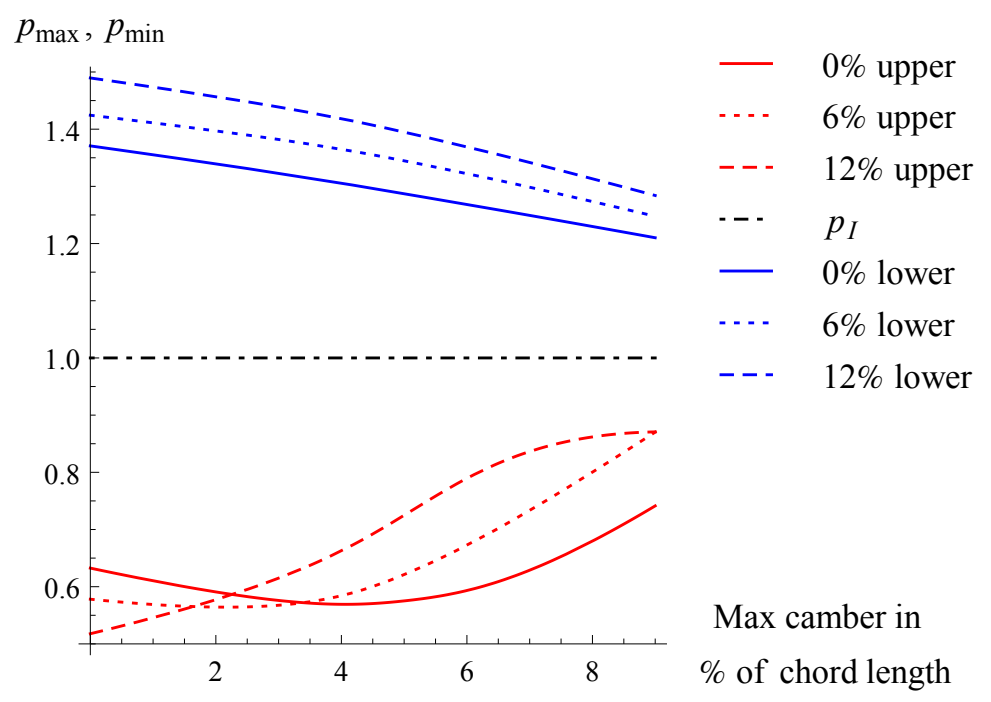

Figure 1.13: Maximum and minimum total far-field pressure (normalised with respect to the incident pressure), as functions of maximum percentage camber,for NACA 4-digit aerofoils with maximum camber at $10 \%$ chord and zero angle of attack. The legend denotes maximum thickness in $\%$ of chord length and the location; either in the upper (shadow) or lower (reflection) Fresnel region. $p_{I}$ denotes the pressure generated by the incident sound wave. 
minimum pressures occur within the reflection and shadow Fresnel regions, respectively. Each maximum and minimum is generated by two Fresnel regions combining in the far field, emanating from the leading and trailing edges of the aerofoil, which have a phase shift between them. This phase shift, $\sigma_{s}$ in (1.5.0.2), is an important factor in determining the nature of the total pressure within the Fresnel regions, and depends strongly on the non-uniform mean flow around the aerofoil.

To illustrate the importance of the phase shift, consider Figure 1.12 and Table 1.1; for $0 \%$ thickness at $\alpha_{i}=-5^{\circ}$, the Fresnel region contributions are almost in and out of phase above and below the flat plate respectively, with the phase shift above the plate being close to 0 , and the shift below being close to $\pi$. Hence above the plate, we subtract the magnitude of the scattered field generated at the trailing edge from the scattered field generated at the leading edge (we subtract when the fields are in phase because there is an overall negative sign difference between the relevant contributions in the leading- and trailing-edge fields in (1.5.0.1)). Below the plate, because the fields are almost out of phase, we end up summing their magnitudes. Thus the upper solid curve (denoting the maximum pressure below the plate) in Figure 1.12 is further from the (unit) amplitude of the incident pressure than the lower solid curve (denoting the minimum pressure above the plate).

As we increase the angle of attack, the phase shift above the plate increases, whilst the phase shift below the plate decreases, and the overall phase shift difference comes closer to 0 , and equals zero at zero angle of attack. In this case, the flat plate is aligned with the steady mean flow, hence we expect that, for any angle of propagation of incident sound wave, the pressure perturbation above the plate mirrors that below the plate. This is precisely the case seen in Figure 1.12 where for any value of thickness, at $\alpha_{i}=0$ the maximum and minimum total pressures are equidistant from unit pressure. As we increase the angle of attack further still, the phase shift above the plate still increases and the phase shift below the plate still decreases, and as these values approach $\pi$ and 0 , we begin adding and subtracting the magnitudes of the pressure from the leadingedge scattered field and the trailing-edge scattered field respectively, which results in the upper curve approaching unit pressure, and the lower curve tending further from unit pressure. This process occurs until $\alpha_{i} \approx 7^{\circ}$ at which point both the maximum and minimum pressure curves approach minimum values (for larger values of $\alpha_{i}$, we begin to veer out of the $\alpha_{i}=O(\epsilon)$ region so the results are less reliable). One anticipates the maximum and minimum pressures will continue to oscillate as we increase the angle of attack, and the troughs of the maximum and minimum pressure curves will occur when the pressure shift difference between them are close to $\pi$, whereas the peaks will occur 
when the difference is close to $-\pi$.

The variation of maximum and minimum pressure for a cambered aerofoil is shown in Figure 1.13. There is symmetry about unit pressure for zero camber for all values of thickness plotted here, because once again the phase shifts between the leading and trailing Fresnel regions are the same above and below the aerofoil, for zero camber. Upon varying the amount of camber, we not only vary the phase shift between the Fresnel regions, we also alter the shape of the leading and trailing edges with respect to the incident flow, and hence get a variation in magnitude of the leading- and trailing-edge scattered pressure fields as well as the variation in phase. Thus there is little symmetry anywhere else in any of the curves in this figure, but it illustrates the complicated nature of the sound scattered in the Fresnel regions as we alter the aerofoil geometry.

\subsection{Conclusions}

This chapter has investigated the scattering of a high-frequency sound wave by an aerofoil in steady mean flow. In particular, a uniformly-valid far-field solution for the scattered acoustic pressure has been found, correct to the leading two terms in both the amplitude and the phase. In the high-frequency limit the far-field sound is dominated by scattering from the leading and trailing edges, and the magnitude of the scattered pressure is greatest in the Fresnel reflection and shadow regions. The existence of these Fresnel regions represents a structural difference between our results and the work of Myers \& Kerschen $(1995,1997)$ and Tsai $(1992)$, who considered an incident gust rather than an incident sound wave. A number of physical insights have arisen from our analysis; first, the difference in refraction experienced by the sound from the leading and trailing edges en-route to the observer, represented by the quantities $\sigma_{s}^{ \pm}$, plays a key role. For instance (in Figures 1.6 and 1.7) the aerofoil camber and mean loading have a direct effect on the number of lobes in the sound field, while (in Figures 1.12 and 1.13) refraction is shown to have a significant effect on the amplitudes in the Fresnel regions (i.e. on the amplitude of the total reflected field and on the depth of the shadow). Secondly, the aerofoil geometry and non-uniform flow close to the leading edge can have an appreciable effect, especially in the Fresnel region (see Figure 1.7, where we vary the thickness with everything else held fixed).

We have also seen that the angle of incidence plays an important role; the sound pressure level of the scattered field increases with sound incidence angle (Figure 1.9), which is seen to be due in part to the increased momentum blocking for oblique sound waves. Further, the character of the sound field changes between cases in which the 
incident sound comes from upstream and from downstream, which is seen to be due to the Fresnel regions dominating the highly oscillatory diffracted field upstream in the latter case (Figure 1.11). The precise implications of this effect for rotor blockage will only become clear once a cascade calculation has been completed, but given that the cascade noise is highly sensitive to the interference of the scattered field from different blades, we can speculate that the possible constructive interference of the upstream Fresnel regions of the different blades could reduce the opacity of a rotor to sound generated downstream.

The results in this chapter are applicable to any aerofoil with a parabolic leading edge, with nose radius scaling with thickness squared, and a sharp trailing edge, although aerofoils with different leading-edge shapes can be analysed using the same steps laid out by this chapter. The parabolic leading edge is convenient for both industrial aerofoils such as the NACA series, and mathematical aerofoils such as the Joukowski aerofoil.

Whilst new sound power is not created during the interaction of the incident field with the aerofoil, it is scattered and redirected by the aerofoil, and understanding this process is of great importance if one wishes to optimise shielding within an aeroengine. A principal benefit of the work presented here is in Section 1.6.2; this analysis allows the location and magnitude of the maximum total pressure obtained during sound-aerofoil interaction to be calculated swiftly and easily. Moreover this maximum can be calculated for a variety of aerofoil geometries and flow conditions with little difficulty, hence optimal aerofoil geometries can quickly be specified. Compare this method to computationally calculating the far-field pressure for many aerofoils (just one such calculation takes a substantial amount of time and processing power), and it becomes clear how this work could be useful to industry. Furthermore, as mentioned in the introduction to this dissertation, current computational schemes only run in low- to mid-range frequencies; high-frequency results are increasingly hard to compute.

It must be noted that the effects of a turbulent boundary layer have been neglected, and it is well-known that the interaction of turbulence with the trailing edge is a very important additional source of sounds (Ffowcs Williams \& Hawkings, 1969; Howe, 1978). Furthermore there is an upper limit of validity of the Kutta condition we apply at the trailing edge, namely $k<O\left(R e^{1 / 4}\right)$, with $R e$ the Reynolds number based on chord length. A detailed discussion of this limit can be found in Crighton (1985). A further and more obvious breakdown of our analysis occurs when the angle of attack approaches the stall angle. For thin aerofoils this restricts the applications of our theory to aerofoils at small angles of attack, but for cascades this restriction is not so severe, given the flow turning induced upstream. 


\section{List of Symbols for Chapter 1}

a constant determining the shape of the aerofoil nose.

$a_{0} \quad$ speed of sound of the steady flow, which far upstream equals $a_{\infty}$.

$C_{ \pm} \quad k \delta M_{\infty}^{2}\left(2 \pm \Gamma / 2+\alpha_{t}\right)$.

$d \quad$ boundary condition term for inner solution $H_{3},=d_{1}+d_{2}+d_{3}$.

$D_{i} \quad$ directivities of the outer limits of $P_{i}$.

$D_{\text {ltr }}$ amplitude function of the camber-dependent leading-edge transition solution.

$D \quad$ directivity function for the far-field acoustics.

$F \quad$ complex potential in $(\phi, \psi)$ space for the steady flow around the aerofoil. $F_{0}$ denotes the complex potential for a flat plate at an angle of attack.

$G \quad$ velocity potential.

$\mathcal{G}$ amplitude function of the thickness-dependent leading-edge transition solution.

$h \quad$ modified velocity potential in the outer region.

$h_{I} \quad$ modified velocity potential of the incident sound wave.

$h_{l s} \quad$ leading-edge transition solution to $h$. Corresponding trailing-edge transition solution is $h_{t s}$.

$H_{t} \quad$ trailing-edge scattered inner solution of $h$, with outer limit directivity, $T$.

$H_{t}^{\text {ref }}$ trailing-edge reflected inner solution of $h, H_{t}^{\text {ref }}=H_{0}^{t} \mathrm{e}^{\mathrm{i} k w \cos \chi\left(2 \pm \Gamma / 2+\alpha_{t}\right)}$.

$H$ leading-edge inner solution of $h$, separated into a flat plate term, $H_{0}$, thickness contributions, $H_{i}$ and camber and angle of attack contributions $P_{i}$.

$k_{1,2} \quad$ wavenumbers of the incident sound wave, $\left(k_{1}, k_{2}\right)=(\cos \chi, \sin \chi)$.

$k \quad$ reduced frequency parameter.

$\boldsymbol{k} \quad$ wave vector in $(\phi, \psi)$ space, $=k\left(k_{1}, k_{2}, k_{3}\right)$.

$L_{i} \quad$ directivities of the outer limits of $H_{i}$.

$L_{0}^{t} \quad$ directivity of the outer limit of $H_{0}^{t}$.

$M_{0} \quad$ Mach number of the uniform flow, which far upstream is $M_{\infty}$.

$p \quad$ modified unsteady pressure.

$q \quad$ velocity variation of the steady flow around the aerofoil. 
$t \quad$ aerofoil thickness, $t=\epsilon t^{\prime}$.

$\boldsymbol{u} \quad$ total flow field generated by the upstream perturbation.

$U_{0} \quad$ steady flow speed, which far upstream equals $U_{\infty}$.

$\boldsymbol{v} \quad$ evolution of the incident disturbance in the steady mean flow.

$V \quad-\beta_{\infty}^{2}\left(1-\frac{(\gamma+1) M_{\infty}^{4}}{2 \beta_{\infty}^{4} w^{2}}(\delta-w \cos \theta)^{2}\right)$.

$w \quad \sqrt{\left(\delta M_{\infty}\right)^{2}-\left(k_{3} / \beta_{\infty}\right)^{2}}$.

$z \quad$ complex coordinate in $(\phi, \psi)$ space.

$\alpha_{\text {eff }}$ effective angle of attack relative to a horizontal uniform flow accounting for camber at the nose, $\alpha_{\text {eff }}=\epsilon \alpha_{\text {eff }}^{\prime}$.

$\alpha_{g} \quad \beta_{\infty} \Gamma /(2 \pi)$.

$\alpha_{i} \quad$ aerofoil angle of attack relative to a horizontal uniform flow, $\alpha_{i}=\epsilon \alpha_{i}^{\prime}$.

$\alpha_{t} \quad \operatorname{Re}\left(\epsilon F^{(t)}(2)\right)$.

$\beta_{0} \quad$ Prandtl-Glauert compressibility factor, $\sqrt{1-M_{0}^{2}}$, which far upstream equals $\beta_{\infty}=$ $\sqrt{1-M_{\infty}^{2}}$.

$\delta \quad \omega / \beta_{\infty}^{2}$.

$\Delta p \quad$ amplitude of the pressure jump across the trailing edge.

$\epsilon \quad$ small asymptotic parameter for aerofoil thickness, camber and angle of attack.

$\Gamma \quad$ steady circulation around the aerofoil.

$\gamma \quad$ ratio of specific heats.

$\mu \quad$ angle variation of the steady flow around the aerofoil.

$\rho_{0} \quad$ density of the steady flow, which far upstream equals $\rho_{\infty}$.

$\phi \quad$ horizontal coordinate corresponding to the modified velocity potential of the steady flow around the aerofoil, with origin at the leading edge.

$\psi \quad$ vertical coordinate corresponding to the streamlines of the steady flow around the aerofoil, with origin at the leading edge.

$\sigma_{I} \quad$ phase of the modified velocity potential of the incident sound wave, $\sigma_{I}=\sigma_{0}+\epsilon \sigma_{1}+$ $O\left(\epsilon^{2}\right)$.

$\sigma \quad$ phase function the outer region, $=\sigma_{l_{0}, t_{0}}+\epsilon \sigma_{l_{1}, t_{1}}$.

$\sigma_{s} \quad$ phase shift between the leading- and the trailing-edge ray fields. 
$\omega \quad$ non-dimensionalised frequency.

$\chi \quad$ angle of propagation of the incident sound wave.

$l \quad$ subscript denotes the function with respect to an origin at the leading edge of the aerofoil.

$t \quad$ subscript denotes the function with respect to an origin at the trailing edge of the aerofoil.

(c) superscript denotes a camber-dependent quantity.

(t) superscript denotes a thickness-dependent quantity.

$u \quad$ superscript denotes uniformly valid approximation.

* $\quad$ superscript denotes a dimensionalised quantity. 


\section{Appendix A}

Upon substituting the solution for $H_{0}$, (1.2.1.3), into the right hand side of (1.2.4.1a) and by using the same method as Tsai $(1992)^{1}$ we find the particular solution of (1.2.4.1a), denoted with a hat, to be

$$
\begin{aligned}
\hat{H}_{3 p}= & \frac{\mathrm{i} \sqrt{w} \sin \chi \beta_{\infty} \tilde{a}}{\pi \sqrt{\cos \chi+1}}\left(\sqrt{R} \cos \frac{\theta}{2} \int_{-\infty}^{\infty} \frac{\sqrt{\lambda-w} \mathrm{e}^{a(\lambda, R, \theta)}}{\lambda+w \cos \chi}\left[1-\frac{(\lambda+\delta)^{2}(\gamma+1) M_{\infty}^{4}}{2 \beta_{\infty}^{4} w^{2}}\right] d \lambda\right. \\
& \left.-\operatorname{isgn}(\Psi) \sqrt{R} \sin \frac{\theta}{2} \int_{-\infty}^{\infty} \frac{\lambda \mathrm{e}^{a(\lambda, R, \theta)}}{\sqrt{\lambda+w}(\lambda+w \cos \chi)}\left[1-\frac{(\lambda+\delta)^{2}(\gamma+1) M_{\infty}^{4}}{2 \beta_{\infty}^{4} w^{2}}\right]\right) d \lambda \\
& -\frac{(\gamma+1) M_{\infty}^{4}}{4 \beta_{\infty}^{4} w^{2}} \frac{\mathrm{i} \cos \theta / 2}{\sqrt{R}} \int_{-\infty}^{\infty} \sqrt{\lambda-w} \frac{(\lambda+\delta)}{(\lambda+w \cos \chi)} \mathrm{e}^{a(\lambda, R, \theta)} d \lambda \\
& \left.+\operatorname{sgn}(\Psi) \frac{(\gamma+1) M_{\infty}^{4}}{4 \beta_{\infty}^{4} w^{2}} \frac{\sin \theta / 2}{\sqrt{R}} \int_{-\infty}^{\infty} \frac{\lambda(\lambda+\delta)}{\sqrt{\lambda+w}(\lambda+w \cos \chi)} \mathrm{e}^{a(\lambda, R, \theta)} d \lambda\right) .
\end{aligned}
$$

This particular solution has singular behaviour at the origin which we can analyse by defining

$$
\begin{aligned}
& I_{2}(R, \theta)=\int_{-\infty}^{\infty} \sqrt{\lambda-w}\left(\frac{1}{(\lambda+w \cos \chi)}-\frac{(\gamma+1) M_{\infty}^{4}}{2 \beta_{\infty}^{4} w^{2}} \frac{(\lambda+\delta)^{2}}{(\lambda+w \cos \chi)}\right) \mathrm{e}^{a(\lambda, R, \theta)} d \lambda, \\
& I_{3}(R, \theta)=\int_{-\infty}^{\infty} \frac{\lambda}{\sqrt{\lambda+w}}\left(\frac{1}{(\lambda+w \cos \chi)}-\frac{(\gamma+1) M_{\infty}^{4}}{2 \beta_{\infty}^{4} w^{2}} \frac{(\lambda+\delta)^{2}}{(\lambda+w \cos \chi)}\right) \mathrm{e}^{a(\lambda, R, \theta)} d \lambda, \\
& I_{4}(R, \theta)=\int_{-\infty}^{\infty} \sqrt{\lambda-w} \frac{(\lambda+\delta)}{(\lambda+w \cos \chi)} \mathrm{e}^{a(\lambda, R, \theta)} d \lambda \\
& I_{5}(R, \theta)=\int_{-\infty}^{\infty} \frac{\lambda}{\sqrt{\lambda+w}} \frac{(\lambda+\delta)}{(\lambda+w \cos \chi)} \mathrm{e}^{a(\lambda, R, \theta)} d \lambda .
\end{aligned}
$$

These represent the integrals in (A.1). There is a clear similarity to the gust case considered by Tsai (1992), so we evaluate these integrals as he does; we can write

$$
\begin{aligned}
I_{2}(R, \theta) & =-2 \pi \sqrt{1+\cos \chi} V(\chi) \mathrm{e}^{\mathrm{i} w R(\cos \chi \cos \theta-|\sin \theta||\sin \chi|)} \\
& +2 \mathrm{e}^{\mathrm{i} w R \cos \theta+\pi \mathrm{i} / 4}\left(I_{21}(R, \theta)+\frac{(\gamma+1) M_{\infty}^{4}}{2 \beta_{\infty}^{4} w^{2}} I_{22}(R, \theta)\right), \\
I_{3}(R, \theta) & =\frac{2 \pi \mathrm{i} \sqrt{w} \cos \chi}{\sqrt{1-\cos \chi}} V(\chi) \mathrm{e}^{\mathrm{i} w R(\cos \chi \cos \theta-|\sin \theta||\sin \chi|)} \\
& +2 \mathrm{e}^{\mathrm{i} w R \cos \theta-\pi \mathrm{i} / 4}\left(I_{31}(R, \theta)+\frac{(\gamma+1) M_{\infty}^{4}}{2 \beta_{\infty}^{4} w^{2}} I_{32}(R, \theta)\right) \\
I_{4}(R, \theta) & =-2 \pi \sqrt{1+\cos \chi}(\delta-w \cos \chi) \mathrm{e}^{\mathrm{i} w R(\cos \chi \cos \theta-|\sin \theta||\sin \chi|)}+2 \mathrm{e}^{\mathrm{i} w R \cos \theta-\pi \mathrm{i} / 4} I_{41}(R, \theta),
\end{aligned}
$$

\footnotetext{
${ }^{1}$ This method is repeated in detail in the first appendix of the next chapter. We have chosen to include full details of the method for that solution because of the errors found in Tsai (1992).
} 


$$
I_{5}(R, \theta)=-\frac{2 \pi \mathrm{i} \sqrt{w} \cos \chi}{\sqrt{1-\cos \chi}}(\delta-w \cos \chi) \mathrm{e}^{\mathrm{i} w R(\cos \chi \cos \theta-|\sin \theta \| \sin \chi|)}+2 \mathrm{e}^{\mathrm{i} w R \cos \theta+\pi \mathrm{i} / 4} I_{51}(R, \theta)
$$

and evaluate in the limit $R \rightarrow 0$ to get

$$
\begin{aligned}
& I_{22} \sim \sqrt{\pi}\left(\frac{3}{4 R^{5 / 2}} \sin \frac{3 \theta}{2}+\frac{\mathrm{i}}{8 R^{3 / 2}}\left[(8 \delta-4 w \cos \chi-5 w) \sin \frac{3 \theta}{2}-3 w \sin \frac{7 \theta}{2}\right]\right) \\
& I_{32} \sim \sqrt{\pi}\left(\frac{3}{4 R^{5 / 2}} \cos \frac{3 \theta}{2}+\frac{\mathrm{i}}{8 R^{3 / 2}}\left[(8 \delta-4 w \cos \chi-5 w) \cos \frac{3 \theta}{2}-3 w \cos \frac{7 \theta}{2}\right]\right) \\
& I_{41} \sim \sqrt{\pi}\left(\frac{1}{2 R^{3 / 2}} \sin \frac{3 \theta}{2}+\frac{\mathrm{i}}{4 \sqrt{R}}\left[-2 w \cos \frac{3 \theta}{2} \sin \theta+4(\delta-w \cos \chi-w) \sin \frac{\theta}{2}\right]\right) \\
& I_{51} \sim \sqrt{\pi}\left(\frac{1}{2 R^{3 / 2}} \cos \frac{3 \theta}{2}+\frac{\mathrm{i}}{4 \sqrt{R}}\left[2 w \sin \frac{3 \theta}{2} \sin \theta+4(\delta-w \cos \chi-w) \cos \frac{\theta}{2}\right]\right) .
\end{aligned}
$$

We have neglected terms that do not create singularities at $R=0$. By summing and simplifying we find that the asymptotic behaviour of this solution as $R \rightarrow 0$ is

$$
\hat{H}_{3 p} \sim \frac{\mathrm{i} \sin \chi a \mathrm{e}^{\mathrm{i} \pi / 4}(\gamma+1) M_{\infty}^{4}}{2 \sqrt{\pi} \beta_{\infty}^{3} w \sqrt{w \cos \chi+w}}\left(\frac{\sin 2 \theta}{R^{2}}+\frac{\mathrm{i} \delta \sin \theta}{R}\right) .
$$

We can approximate the terms in (1.2.4.1a) for small $R$, and then integrate the resulting equation to give us a local solution of (1.2.4.1a) valid for small $R$. This informs us that $H_{3}$ ought not be singular at $R=0$. Hence we add the appropriate Hankel functions, which are complementary solutions to the Helmholtz equation, to eliminate the singularity to yield

$$
H_{3 p}=\hat{H}_{3 p}+\frac{a \sin \chi \mathrm{e}^{\mathrm{i} \pi / 4}(\gamma+1) M_{\infty}^{4} \sqrt{\pi}}{4 \beta_{\infty}^{3} w^{3 / 2} \sqrt{\cos \chi+1}}\left(\frac{w}{2} \mathrm{H}_{2}^{(1)}(w R) \sin 2 \theta+\mathrm{i} \delta \mathrm{H}_{1}^{(1)}(w R) \sin \theta\right),
$$

where $H_{1,2}^{(1)}$ are Hankel functions of the first kind (Abramowitz \& Stegun, 1964, pp. 358). We define $H_{3 p}^{*}=H_{3 p}-\hat{H}_{3 p}$. 


\section{Appendix B}

Equation (1.2.4.3c) is factored as $d_{1}+d_{2}+d_{3}$, where

$$
\begin{aligned}
& d_{1}(\Phi)=-\frac{\beta_{\infty} a}{w} \mathrm{e}^{\mathrm{i} w \cos \chi \Phi}\left(-2 w^{2} \sin ^{2} \chi \sqrt{\Phi}-\frac{\mathrm{i} w \cos \chi}{\sqrt{\Phi}}-\right. \\
& \frac{\mathrm{i}(\gamma+1) M_{\infty}^{4}}{2 \beta_{\infty}^{4} w^{2}} \frac{(\delta-w \cos \chi)}{2}\left[-4 \sqrt{\Phi}(\delta-w \cos \chi) w^{2} \sin ^{2} \chi\right. \\
& \left.\left.+\frac{2 \mathrm{i}\left(w \cos \chi(\delta-2 w \cos \chi)+w^{2}\right)}{\sqrt{\Phi}}\right]\right) \\
& d_{2}(\Phi)=\frac{\mathrm{i} w \sin \chi \beta_{\infty} a \mathrm{e}^{\mathrm{i} w \Phi+\mathrm{i} \pi / 4}}{\sqrt{\pi} \sqrt{w \cos \chi+w}} \frac{1}{\cos \chi-1}\left(1-\frac{(\gamma+1) M_{\infty}^{4}}{2 \beta_{\infty}^{4} w^{2}}(\delta-w)^{2}\right) \frac{1}{\Phi}, \\
& d_{3}(\Phi)=\frac{\mathrm{i} w \sin \chi \beta_{\infty} a \mathrm{e}^{\mathrm{i} w \Phi+\mathrm{i} \pi / 4}}{\sqrt{\pi} \sqrt{w \cos \chi+w}}\left[2 \mathrm{i}(w \cos \chi+w)\left(1-\frac{(\gamma+1) M_{\infty}^{4}}{2 \beta_{\infty}^{2} w^{2}}(\delta-w)^{2}\right)-\frac{X}{\Phi}\right. \\
& +\frac{\sqrt{\pi}(\gamma+1) M_{\infty}^{4} \cos \chi}{4 \beta_{\infty}^{4} w \sqrt{w \cos \chi-w} \Phi^{3 / 2}}(\delta-w \cos \chi) \mathrm{e}^{\mathrm{i}(w \cos \chi-w) \Phi-\pi \mathrm{i} / 4} \\
& -\frac{(\gamma+1) M_{\infty}^{4}}{2 \beta_{\infty}^{4} w^{2}}\left(\frac{2}{\Phi^{3}}+\frac{\mathrm{i}(\delta-2 w)}{\Phi^{2}}\right) \\
& +\frac{\sqrt{\pi} \mathrm{e}^{\mathrm{i}(w \cos \chi-w) \Phi-\mathrm{i} \pi / 4}}{\sqrt{w \cos \chi-w}} \operatorname{erfc}\left(\mathrm{e}^{\mathrm{i} \pi / 4} \sqrt{(w \cos \chi-w) \Phi}\right)\left[-2 w^{2} \sin ^{2} \chi \sqrt{\Phi}\right. \\
& -\frac{\mathrm{i} w \cos \chi}{\sqrt{\Phi}}-\frac{\mathrm{i}(\gamma+1) M_{\infty}^{4}}{4 \beta_{\infty}^{4} w^{2}}(\delta-w \cos \chi)\left(-4 \sqrt{\Phi}(\delta-w \cos \chi) w^{2} \sin ^{2} \chi\right. \\
& \left.\left.\left.+\frac{2 i\left((\delta-2 w \cos \chi) w \cos \chi+w^{2}\right)}{\sqrt{\Phi}}+\frac{w \cos \chi}{\Phi^{3 / 2}}\right)\right]\right] \\
& +\frac{a M_{\infty}^{2} \mathrm{e}^{\mathrm{i} w \cos \chi \Phi}}{2 \beta_{\infty} \Phi^{3 / 2}} \operatorname{erf}\left(\mathrm{e}^{\mathrm{i} \pi / 4} \sqrt{(w \cos \chi-w) \Phi}\right) \\
& -\frac{(\gamma+1) M_{\infty}^{4} \sin \chi a \sqrt{\pi}}{4 \beta_{\infty}^{3} \sqrt{w \cos \chi+w}} \mathrm{e}^{\pi \mathrm{i} / 4}\left(w H_{2}^{(1)}(w \Phi)+\mathrm{i} \delta H_{1}^{(1)}(w \Phi)\right) \frac{1}{\Phi},
\end{aligned}
$$

and

$$
X=\frac{1}{\cos \chi-1}\left(1+\frac{(\gamma+1) M_{\infty}^{4}}{2 \beta_{\infty}^{4} w^{2}}\left[w(\cos \chi-1)^{2}(w \cos \chi+w-\delta)+(\delta-w)^{2}\right]\right) .
$$

We assume that $\delta$ has a small positive imaginary part so that when we apply the Fourier transform, our integrals converge at infinity. The $F_{+i}$ required for the evaluation of the $L_{3 c_{i}}$ in Section 1.2 .4 are given by

$$
F_{+i}=\int_{0}^{\infty} \frac{d_{i}(x) \mathrm{e}^{\mathrm{i} \lambda x}}{\sqrt{\lambda-w}} \operatorname{erf}\left(\mathrm{e}^{\mathrm{i} \pi / 4} \sqrt{(\lambda-w) x}\right) d x
$$


We evaluate $F_{+1,2}$, noting that the formula

$$
F_{+i}(\lambda)=\frac{1}{2 \pi \mathrm{i}} \int_{C_{1}} \frac{d \kappa}{\sqrt{\kappa-w}(\kappa-\lambda)} \int_{0}^{\infty} d_{i}(x) \mathrm{e}^{\mathrm{i} \kappa x} d x
$$

(where the contour of integration, $C_{1}$, runs parallel to, but just above, the real $\kappa$ axis, and below $w$ ), is more appropriate to evaluate $F_{+1,2}$ since it allows us to properly assess the effects of the branch cuts. For $F_{+1}$ we evaluate the $x$ integral in (B.6) first, whereas for $F_{2+}$ is it easier to evaluate the $\kappa$ integral first. We obtain

$$
\begin{aligned}
F_{+1}= & \frac{-\mathrm{i} \beta_{\infty} a \mathrm{e}^{3 \mathrm{i} \pi / 4}}{\sqrt{\pi}}\left(\frac{-\mathrm{i}(\alpha-w b \cos \chi)}{(w+w \cos \chi)(\lambda+w \cos \chi)}\right. \\
& \left.-\frac{\pi}{2} \frac{\alpha+\lambda b}{\sqrt{\lambda-w}(w \cos (\chi)+\lambda)^{3 / 2}}\left[1-\frac{2}{\pi} \arcsin \left(\frac{\sqrt{w-\lambda}}{\sqrt{w+w \cos \chi}}\right)\right]\right),
\end{aligned}
$$

where

$$
\begin{aligned}
\alpha & =-2 w^{2}+\frac{\mathrm{i} M_{\infty}^{4}(\gamma+1)}{\beta_{\infty}^{2}}(\delta-w \cos \chi)^{2} \\
b & =-2 w \cos \chi-\frac{\mathrm{i} M_{\infty}^{4}(\gamma+1)}{\beta_{\infty}^{2} w}(\delta-w \cos \chi)(\cos \chi(\delta-2 w \cos \chi)+w),
\end{aligned}
$$

and

$$
F_{+2}=\frac{2 \mathrm{i} \sin \chi \sqrt{w} \beta_{\infty} a \mathrm{e}^{\mathrm{i} \pi / 4}}{\sqrt{\pi} \sqrt{\cos \chi+1} \sqrt{\lambda-w}} \frac{1}{\cos \chi-1}\left(1-\frac{(\gamma+1) M_{\infty}^{4}}{2 \beta_{\infty}^{4} w^{2}}(\delta-w)^{2}\right) \log \left[\frac{\sqrt{2 w}-\mathrm{i} \sqrt{\lambda-w}}{\sqrt{\lambda+w}}\right] .
$$

The decomposition of the complementary solution is done in this way so that we may explicitly evaluate $L_{3 c_{1}}$ and $L_{3 c_{2}}$. The remaining terms for the integral involved in $L_{3 c_{3}}$ is convergent, but must be evaluated numerically. We take the outer limits of the $H_{3 c_{i}}$ given by $(1.2 .4 .4 \mathrm{~b})$ to find the directivity functions $L_{3 c_{i}}$. We find

$$
\begin{aligned}
L_{3 c_{1}}(\theta)= & -\frac{\beta_{\infty} a \operatorname{sgn}(\Psi) \cos (\theta / 2)}{2 \sqrt{2} w(\cos \chi-\cos \theta)}\left[\frac{\alpha-w b \cos \theta}{w \sqrt{\cos \chi-\cos \theta}}\left(1-\frac{2}{\pi} \arcsin \left[\frac{\sqrt{1+\cos \theta}}{\sqrt{1+\cos \chi}}\right]\right)\right. \\
& \left.-\frac{2}{\pi} \frac{(\alpha-w \cos (\chi) b) \sqrt{1+\cos \theta}}{w \cos \chi+w}\right],
\end{aligned}
$$

This is not singular at $\cos \theta=\cos \chi$ which can be seen by taking a Taylor series expansion for $\theta$ close to $\chi$. In Ayton \& Peake (2013) we incorrectly thought $L_{3 c_{1}}$ was singular at $\cos \theta=\cos \chi$ and presented a complementary error function style uniformly-valid solution, which given the parameter $\beta,(1.2 .1 .7 \mathrm{~b}$ ), would never be $O(1)$ (as we have now 
established that there is no singularity) is equivalent to the result presented here.

The uniformly-valid expansion of $L_{3 c_{2}}$ found by using Van der Waerden's method (Van der Waerden, 1952) ${ }^{1}$ is

$$
\begin{gathered}
L_{3 c_{2}}^{u}(r, \theta)=\frac{\sqrt{2} \operatorname{sgn}(\Psi) \sin \chi \beta_{\infty} a}{\pi \sqrt{\cos \chi+1}} \frac{1}{\cos \chi-1}\left(1-\frac{(\gamma+1) M_{\infty}^{4}}{2 \beta_{\infty}^{4} w^{2}}(\delta-w)^{2}\right) \\
{\left[\frac{\sqrt{2}}{\sqrt{\pi} \sqrt{1+\cos \theta}}\left(\frac{\sqrt{\pi}}{2} \log (k r)-\int_{-\infty}^{\infty} \log \left(s+\mathrm{e}^{\mathrm{i} \pi / 4} \sqrt{w(1-\cos \theta) k r}\right) \mathrm{e}^{-s^{2}} d s\right)\right.} \\
\left.+\log \left(\frac{\sqrt{2}+\sqrt{1+\cos \theta}}{\sqrt{1-\cos \theta}}\right)+\frac{\log (1-\cos \theta)}{\sqrt{2} \sqrt{1+\cos \theta}}+\frac{i \pi+2 \log (w)}{2 \sqrt{2} \sqrt{1+\cos \theta}}\right]
\end{gathered}
$$

so that

$$
H_{3 c_{2}}^{u} \sim L_{3 c_{2}}^{u}(r, \theta) \frac{\mathrm{e}^{\mathrm{i} k w r}}{\sqrt{k r}} .
$$

The logarithmic singularity at $\theta=0$ has now been removed, as the singular contributions of the second and third to last terms in (B.10) cancel. This singularity occurs originally at $\theta=O\left(k^{-1 / 2}\right)$ in the integral expression of the $H_{3 c_{2}}$ Wiener-Hopf solution (see (B.8)), which we shall see later is a key scaling for the leading-edge transition solution. Finally

$$
L_{3 c_{3}}(\theta)=-\frac{\operatorname{sgn}(\psi) \mathrm{e}^{\mathrm{i} \pi / 4}}{\sqrt{2 \pi w}} \int_{0}^{\infty} \mathrm{e}^{-\mathrm{i} w \cos \theta x} \operatorname{erf}\left(\mathrm{e}^{-\mathrm{i} \pi / 4} \sqrt{w(1+\cos \theta) x}\right) d_{3}(x) d x
$$

is retained in integral form as it is not possible to find an analytic expression for this integral; it must be evaluated numerically.

\footnotetext{
${ }^{1}$ Again, full details of this method will be covered in the first appendix of the next chapter because of errors in Tsai's results.
} 


\section{Appendix C}

The thickness-related total leading-edge solution for $\phi>2$ on $\psi=0_{ \pm}$is given by $h_{l}^{(t)}\left(\phi, 0_{ \pm}\right)+h_{l s}^{(t)}\left(\phi, 0_{ \pm}\right)$, using the expression for $G$ as given in (1.3.2.5b), so

$$
\begin{array}{r}
h_{l}^{u(t)}\left(\phi, 0_{ \pm}\right)=\frac{\mathrm{e}^{\mathrm{i} k w \sigma_{l}(\phi, 0)}}{\sqrt{k \phi}}\left(L_{0}\left(0_{ \pm}\right)+t \sqrt{k}\left[L_{1}\left(0_{ \pm}\right)+L_{2}\left(0_{ \pm}\right)+L_{3 p}\left(0_{ \pm}\right)+L_{3 c_{1}}\left(0_{ \pm}\right)\right.\right. \\
+L_{3 c_{3}}\left(0_{ \pm}\right) \mp \frac{\beta_{\infty} \sin \chi}{\sqrt{2} \pi \sqrt{\cos \chi+1}(\cos \chi-1)}\left(1-\frac{(\gamma+1) M_{\infty}^{4}}{2 \beta_{\infty}^{4} w^{2}}(\delta-w)^{2}\right)\left\{\int _ { 0 } ^ { 2 } \left[\frac{a}{\xi}\right.\right. \\
\left.+\frac{\beta_{\infty}}{t^{\prime} \xi^{3 / 2}} \int_{0}^{\xi} \frac{\partial q^{(t)}}{\partial \theta}\left(r^{\prime}, 0\right) d r^{\prime}\right] \frac{\sqrt{\phi}}{\sqrt{\phi-\xi}} d \xi+\int_{2}^{\phi}\left[\frac{a}{\xi}+\frac{\beta_{\infty}}{t^{\prime} \xi^{3 / 2}} \int_{0}^{2} \frac{\partial q^{(t)}}{\partial \theta}\left(r^{\prime}, 0\right) d r^{\prime}\right] \frac{\sqrt{\phi}}{\sqrt{\phi-\xi}} d \xi \\
\left.\left.\left.-\frac{a}{2}\left(\log [32 k \phi w]+\tilde{\gamma}-\frac{\pi \mathrm{i}}{2}\right)\right\}\right]\right) .
\end{array}
$$

The final term in this expression occurs due to the contribution from the non-uniformity in $L_{3 c_{2}}$ at $\theta=0_{ \pm}$and can be seen by setting $\theta=0$ in (B.10), using Gradshteyn \& Ryzhik (1980, p. 574). Equation (C.1) forms the basis of the pressure jump across the trailing edge, which is given by

$$
\begin{aligned}
& \left.p_{l}\right|_{\psi=0+}-\left.p_{l}\right|_{\psi=0-}=\frac{\mathrm{i}(\delta-w)}{\sqrt{k} \sqrt{2+\phi_{t}}} \mathrm{e}^{\mathrm{i} k\left(w-\delta M_{\infty}^{2}\right)\left(2+\phi_{t}+\alpha_{t}\right)+\mathrm{i} k w \epsilon V(0) \int_{0}^{\phi} q\left(r^{\prime}, 0\right) d r^{\prime}+\mathrm{i} k w \epsilon \sigma_{1}(0,0)} \\
& {\left[\mathrm { e } ^ { \mathrm { i } k ( w - \delta M _ { \infty } ^ { 2 } ) \Gamma / 2 } \left\{P_{l}(0)+t \sqrt{k}\left[L_{1}(0)+L_{2}(0)+L_{3 p}(0)+L_{3 c_{1}}(0)+L_{3 c_{2}}(0)+L_{3 c_{3}}(0)\right]\right.\right.} \\
& -t \sqrt{k} \frac{\sin \chi \beta_{\infty}}{\sqrt{2} \pi \sqrt{\cos \chi+1}(\cos \chi-1)}\left(1-\frac{(\gamma+1) M_{\infty}^{4}}{2 \beta_{\infty}^{4} w^{2}}(\delta-w)^{2}\right)\left\{\int _ { 0 } ^ { 2 } \left[\frac{a}{\xi}\right.\right. \\
& \left.+\frac{\beta_{\infty}}{t^{\prime} \xi^{3 / 2}} \int_{0}^{\xi} \frac{\partial q^{(t)}}{\partial \theta}\left(r^{\prime}, 0\right) d r^{\prime}\right] \frac{\sqrt{2+\phi_{t}}}{\sqrt{2+\phi_{t}-\xi}} d \xi+\int_{2}^{2+\phi_{t}}\left[\frac{a}{\xi}+\frac{\beta_{\infty}}{t^{\prime} \xi^{3 / 2}} \int_{0}^{2} \frac{\partial q^{(t)}}{\partial \theta}\left(r^{\prime}, 0\right) d r^{\prime}\right] \\
& \left.\left.\left.\frac{\sqrt{2+\phi_{t}}}{\sqrt{2+\phi_{t}-\xi}} d \xi-\frac{a}{2}\left(\log \left[32 k\left(\phi_{t}+2\right) w\right]+\tilde{\gamma}-\frac{\pi \mathrm{i}}{2}\right)\right\}\right]\right\}-\left[\mathrm { e } ^ { - \mathrm { i } k ( w - \delta M _ { \infty } ^ { 2 } ) \Gamma / 2 } \left\{P_{l}(2 \pi)\right.\right. \\
& +t \sqrt{k}\left[L_{1}(2 \pi)+L_{2}(2 \pi)+L_{3 p}(2 \pi)+L_{3 c_{1}}(2 \pi)+L_{3 c_{2}}(2 \pi)+L_{3 c_{3}}(2 \pi)\right] \\
& +t \sqrt{k} \frac{\sin \chi \beta_{\infty}}{\sqrt{2} \pi \sqrt{\cos \chi+1}(\cos \chi-1)}\left(1-\frac{(\gamma+1) M_{\infty}^{4}}{2 \beta_{\infty}^{4} w^{2}}(\delta-w)^{2}\right) \\
& \left\{\int_{0}^{2}\left[\frac{a}{\xi}+\frac{\beta_{\infty}}{t^{\prime} \xi^{3 / 2}} \int_{0}^{\xi} \frac{\partial q^{(t)}}{\partial \theta}\left(r^{\prime}, 0\right) d r^{\prime}\right] \frac{\sqrt{2+\phi_{t}}}{\sqrt{2+\phi_{t}-\xi}} d \xi\right. \\
& +\int_{2}^{2+\phi_{t}}\left[\frac{a}{\xi}+\frac{\beta_{\infty}}{t^{\prime} \xi^{3 / 2}} \int_{0}^{2} \frac{\partial q^{(t)}}{\partial \theta}\left(r^{\prime}, 0\right) d r^{\prime}\right] \frac{\sqrt{2+\phi_{t}}}{\sqrt{2+\phi_{t}-\xi}} d \xi \\
& \left.\left.\left.-\frac{a}{2}\left(\log \left[32 k\left(\phi_{t}+2\right) w\right]+\tilde{\gamma}-\frac{\pi \mathrm{i}}{2}\right)\right\}\right]\right\}+\epsilon \sqrt{k} \frac{\mathrm{e}^{\pi \mathrm{i} / 4} i V(0)}{\sqrt{\pi w} \beta_{\infty}} \int_{0}^{2} \frac{\xi y^{(c) \prime}(\xi)-y^{(c)}(\xi)}{\sqrt{2-\xi} \xi^{3 / 2}} d \xi \\
& \left.\left(\mathrm{e}^{\mathrm{i} k\left(w-\delta M_{\infty}^{2}\right) \Gamma / 2} P_{l}(0)+\mathrm{e}^{-\mathrm{i} k\left(w-\delta M_{\infty}^{2}\right) \Gamma / 2} P_{l}(2 \pi)\right)\right] .
\end{aligned}
$$


We write the pressure jump in the form (1.4.2.2) where

$$
\begin{aligned}
& \Delta p^{(t)}\left(\phi_{t}\right)=\frac{\sqrt{2}}{\sqrt{2+\phi_{t}}} \Delta p^{(t)}(0)+t \sqrt{k} \frac{\mathrm{i} w \sin \chi \beta_{\infty} \sqrt{w}}{\sqrt{2} \pi \sqrt{\delta+w}} \mathrm{e}^{\mathrm{i} k\left(w-\delta M_{\infty}^{2}\right)\left(2+\alpha_{t}\right)+\mathrm{i} k w \epsilon \sigma_{1}^{t}(0,0)} \\
& \left(1-\frac{(\gamma+1) M_{\infty}^{4}}{2 \beta_{\infty}^{4} w^{2}}(\delta-w)^{2}\right)\left\{\mathrm { e } ^ { \mathrm { i } k ( w - \delta M _ { \infty } ^ { 2 } ) \Gamma / 2 } \mathrm { e } ^ { \mathrm { i } k w \epsilon \sigma _ { l 1 } ( 2 , 0 ) } \left(\int _ { 0 } ^ { 2 } \left[\frac{a}{\xi}\right.\right.\right. \\
& \left.+\frac{\beta_{\infty}}{t^{\prime} \xi^{3 / 2}} \int_{0}^{\xi} \frac{\partial q^{(t)}}{\partial \theta}\left(r^{\prime}, 0\right) d r^{\prime}\right]\left(\frac{1}{\sqrt{2+\phi_{t}-\xi}}-\frac{\sqrt{2}}{\sqrt{2-\xi} \sqrt{2+\phi_{t}}}\right) d \xi \\
& \left.-\frac{a}{\sqrt{2} \sqrt{2+\phi_{t}}} \log \left[\frac{\phi_{t}+2}{2}\right]+\int_{2}^{2+\phi_{t}}\left[\frac{a}{\xi}+\frac{\beta_{\infty}}{t^{\prime} \xi^{3 / 2}} \int_{0}^{2} \frac{\partial q^{(t)}}{\partial \theta}\left(r^{\prime}, 0\right) d r^{\prime}\right] \frac{1}{\sqrt{2+\phi_{t}-\xi}} d \xi\right) \\
& +\mathrm{e}^{-\mathrm{i} k\left(w-\delta M_{\infty}^{2}\right) \Gamma / 2} \mathrm{e}^{\mathrm{i} k w \epsilon \sigma_{l 1}(2,2 \pi)}\left(\int_{0}^{2}\left[\frac{a}{\xi}+\frac{\beta_{\infty}}{t^{\prime} \xi^{3 / 2}} \int_{0}^{\xi} \frac{\partial q^{(t)}}{\partial \theta}\left(r^{\prime}, 2 \pi\right) d r^{\prime}\right]\right. \\
& \left(\frac{1}{\sqrt{2+\phi_{t}-\xi}}-\frac{a}{\sqrt{2-\xi} \sqrt{2+\phi_{t}}}\right) d \xi-\frac{a}{\sqrt{2} \sqrt{2+\phi_{t}}} \log \left[\frac{\phi_{t}+2}{2}\right] \\
& \left.\left.+\int_{2}^{2+\phi_{t}}\left[\frac{a}{\xi}+\frac{\beta_{\infty}}{t^{\prime} \xi^{3 / 2}} \int_{0}^{2} \frac{\partial q^{(t)}}{\partial \theta}\left(r^{\prime}, 2 \pi\right) d r^{\prime}\right] \frac{1}{\sqrt{2+\phi_{t}-\xi}} d \xi\right)\right\} .
\end{aligned}
$$




\section{Chapter 2}

\section{High-Frequency Sound Generated by Gust-Aerofoil Interaction in Subsonic Uniform Flow}

We now consider gust-aerofoil interaction in steady subsonic uniform flow. In this chapter we extend the work of Myers \& Kerschen (1997) and Tsai (1992) to evaluate the farfield sound generated by gust-aerofoil interaction in uniform flow, for an aerofoil with small thickness, camber and angle of attack, as illustrated in Figure 0.7. Tsai considered only symmetric Joukowski aerofoils at zero angle of attack, whereas Myers and Kerschen considered zero-thickness, cambered plates at non-zero angles of attack. Here we combine all of these features. Much of the analysis is the same as in the previous chapter; we assume the frequency of the incident gust is high, $k \gg 1$, and the thickness and camber angle are small, $\epsilon t^{\prime}, \epsilon \alpha^{\prime} \ll 1$. We again impose the preferred scaling $\epsilon k=O(1)$, and use matched asymptotic expansions to split the problem into similar regions as described for the sound-aerofoil interaction problem; these regions can be seen in Figure 2.1.

We notice that the main difference between the regions arising from gust-aerofoil interaction and sound-aerofoil interaction (in Figure 1.1) is that there are no Fresnel regions occurring for gust-aerofoil interaction. This is the case because a sound wave can directly reflect off the aerofoil surface, and the Fresnel regions occur along the shadow and reflection boundaries for the incident sound wave, where the sound wave is scattered by an edge. A gust has no such shadow and reflection boundaries since itself imposes no pressure fluctuations in the (uniform) flow. Conversely, a feature of the perturbation field that exists for gust-aerofoil interaction, but not for sound-aerofoil interaction, is the hydrodynamic field. This field occurs due to the vorticity source term in the flow field interacting with the solid surface in a way that does not result in acoustic propagation to the far field. A hydrodynamic term decays exponentially away from the surface of the aerofoil, and hence no pressure fluctuations are present in the far field from these such contributions. There were no hydrodynamic terms in the previous chapter because an incident sound wave does not introduce fluctuations that are "frozen in the flow", as illustrated by a zero source term in (1.1.2.4). 


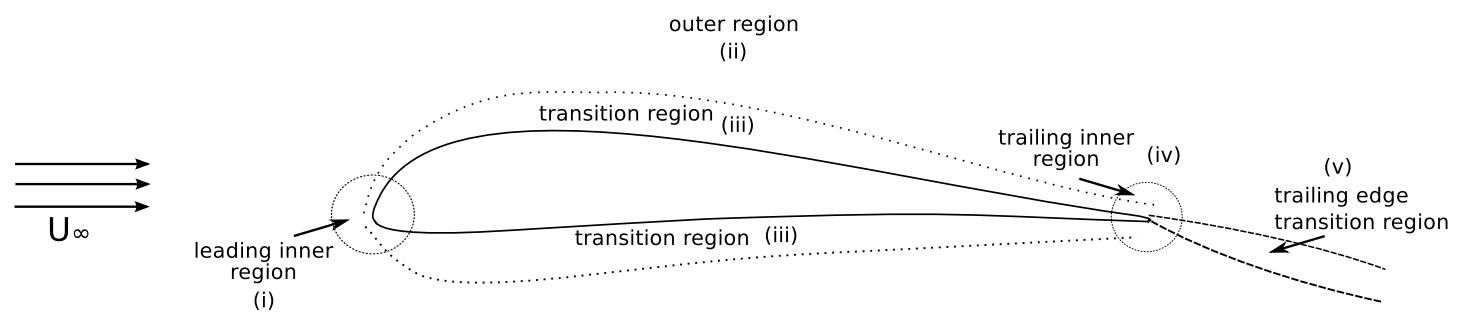

(a) In $(x, y)$ space.

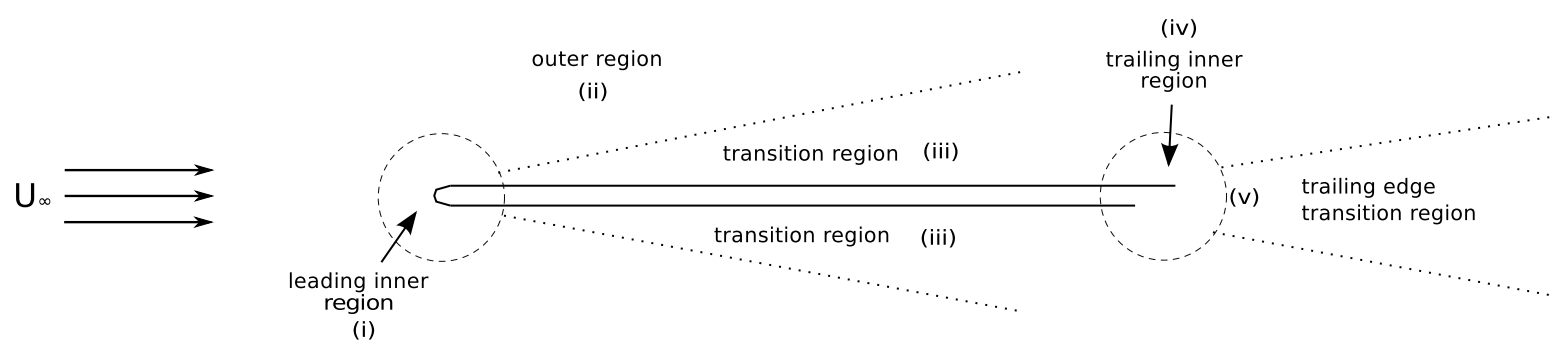

(b) In $(\phi, \psi)$ space.

Figure 2.1: Asymptotic regions around the aerofoil; leading- and trailing-edge inner regions, (i) and (iv), scale as $O\left(k^{-1}\right)$, and the width of the transition regions, (iii) scale as $O\left(k^{-1 / 2}\right)$. The outer region (ii) is $O(1)$. We solve for (i) in Section 2.2, and solve for (ii) in Section 2.3. Region (iii) is solved for in Section 2.4, and regions (iv) and (v) are solved for in Sections 2.5 and 2.6 respectively.

Much of the analysis required to solve the gust-aerofoil problem is similar to that presented in the previous chapter, albeit the boundary conditions on the aerofoil surface are different. For this reason, the work presented in this chapter is not as detailed as in the previous chapter. Variables in this chapter are defined as the corresponding variables to those given in Chapter 1, e.g. the gust reduced frequency here is $k$, corresponding to the previous definition of $k$ as the reduced frequency of the incident sound wave in Chapter 1.

We divide the velocity field for the total unsteady flow in the system into two parts; the gust-solution, which is purely convected, has zero divergence, and no associated pressure fluctuations, and an irrotational field which contains all of the pressure fluctuations. This is the typical decomposition of the unsteady flow field as dictated by rapid distortion theory (Goldstein, 1978b). The fluid rotation is held within the gust hence this is often referred to as the vortical velocity. In a compressible flow the irrotational component contains acoustic waves that propagate at the speed of sound relative to the fluid, and hydrodynamic terms that are convected by the mean flow. Hydrodynamic terms were not found in Chapter 1 since for sound-aerofoil interaction the upstream perturbation is purely acoustic. 
We additionally can include entropy fluctuations that may be imposed on the flow in this new problem. These are decoupled from the velocity and pressure but do produce density fluctuations, and they are also "frozen in the flow" as they are convected at the mean flow speed. The analysis here allows for an arbitrary upstream entropy perturbation, however the example results presented are for purely vortical perturbations and no entropic fluctuations.

In Section 2.1 we set out the governing equations for this new problem, and identify how and why they differ from the governing equations for sound-aerofoil interaction. In Section 2.2 we construct the leading-edge inner solution, using similar techniques as for the sound-aerofoil interaction problem. We identify mathematically why no Fresnel regions emanate from here specifically in Section 2.2.1. We give the hydrodynamic and the leading- and trailing-edge acoustic outer solutions in Section 2.3. Section 2.4 contains analysis of the leading-edge transition solution on the surface of the aerofoil. In Section 2.5 we solve for the trailing-edge inner solution, in Section 2.6 we discuss the trailingedge transition solution, and in Section 2.7 we give the total far-field solution. Section 2.8 contains results and Section 2.9 contains concluding remarks.

\subsection{Formulation of the Governing Equations}

We consider aerofoils with small thickness, camber, and angle of attack, as described in Section 1.1 and set up the problem similarly. The perturbation to the uniform flow due to the presence of the aerofoil is still given by (1.1.1.2), and all variables relating to the geometry of the aerofoil and the background flow are as defined in the previous chapter. We also non-dimensionalise variables in the same way as before. The rapid distortion theory equation of motion remains (1.1.2.2), with $G$ being related to $h$, the modified velocity potential, via (1.1.2.1). In this chapter, the perturbation to the steady mean flow is given by $\boldsymbol{u}=\boldsymbol{v}+\boldsymbol{\nabla} G$, where $\boldsymbol{v}$ is now non-zero and describes the evolution of the incident vortical disturbance, and $G$ is the response to the gust that contains all the pressure fluctuations within the flow. We can also consider entropic disturbances, denoted by $s$, which can be contained within the non-acoustic term $\boldsymbol{v}$.

The gust and entropy perturbation (non-dimensionalised with respect to the specific heat at constant pressure, $c_{p}$ ), evolve in the uniform flow according to the governing 
equations

$$
\begin{array}{ll} 
& \frac{D_{0} s}{D t}=0, \\
& \boldsymbol{v}=\frac{s}{2} \boldsymbol{U}_{0}+\boldsymbol{v}^{*}, \\
\text { with } & \frac{D_{0} \boldsymbol{v}^{*}}{D t}+\boldsymbol{v}^{*} \cdot \boldsymbol{\nabla} \boldsymbol{U}_{0}=0, \\
\text { and } & \frac{D_{0}}{D t}=\frac{\partial}{\partial t}+\boldsymbol{U}_{0} \cdot \boldsymbol{\nabla},
\end{array}
$$

which are derived in Goldstein (1978b) by linearising the Euler equations about the steady background flow around the aerofoil, $\boldsymbol{U}_{0}$, which contains the steady perturbation velocity due to the presence of the aerofoil, $q$, and far upstream is uniform, $\boldsymbol{U}_{0} \rightarrow(1,0,0)$.

First we must solve for the evolution of $\boldsymbol{v}$ and $s$ for a specified upstream disturbance, which follows the analysis in Myers (1987). By noting that the governing equations are linear and the mean flow is uniform far upstream, we can represent an arbitrary disturbance as a superposition of harmonic waves in potential-streamline space, denoted once again by $(\phi, \psi)$ coordinates. Consider therefore

$$
\left.\left(\begin{array}{c}
\boldsymbol{v} \\
s
\end{array}\right)\right|_{\phi \rightarrow-\infty}=\left(\begin{array}{c}
\left(A_{t}, A_{n}, A_{3}\right) \\
2 B
\end{array}\right) \mathrm{e}^{\mathrm{i} k\left(k_{t} \phi+k_{n} \psi+k_{3} \chi-k_{t} t\right)}
$$

where $\boldsymbol{A}=\left(A_{t}, A_{n}, A_{3}\right)$ and $\boldsymbol{k}=\left(k_{t}, k_{n}, k_{3}\right)$ are the velocity amplitude and wavevector components in $\left(\phi, \psi, x_{3}\right)$ space, and $B$ is the amplitude of the entropy fluctuations in $\left(\phi, \psi, x_{3}\right)$ space. Far upstream where the flow is uniform the $\left(\phi, \psi, x_{3}\right)$ coordinates coincide with the Cartesian coordinates $\left(x, \beta_{\infty} y, x_{3}\right)$, and the gust is solenoidal in uniform mean flow, so we must have

$$
A_{t} k_{t}+A_{n} k_{n} \beta_{\infty}+A_{3} k_{3}=0
$$

The extra $\beta_{\infty}$ factor in (2.1.0.3) is due to the Prandtl-Glauert transformation (recall Section 1.1 where we discussed the change from physical coordinates to $(\phi, \psi)$ space).

By writing $\boldsymbol{v}(\phi, \psi, \chi, t)=\left(v_{t}, v_{n}, v_{3}\right)$, Kerschen \& Balsa (1981) derive the solution to (2.1.0.1) with initial condition (2.1.0.2) to be

$$
\begin{aligned}
& v_{t}=\left(\frac{A_{t}^{*}}{U_{0}}+B U_{0}\right) \mathrm{e}^{\mathrm{i} k \sigma} \\
& v_{n}=\rho_{0} U_{0}\left(A_{n}+\beta_{\infty} A_{t}^{*} \frac{\partial g}{\partial \psi}\right) \mathrm{e}^{\mathrm{i} k \sigma},
\end{aligned}
$$




$$
\begin{aligned}
v_{3} & =A_{3} \mathrm{e}^{\mathrm{i} k \sigma} \\
s & =2 B \mathrm{e}^{\mathrm{i} k \sigma} \\
\sigma & =k_{t} \phi+k_{t} \epsilon g(\phi, \psi)+k_{n} \psi+k_{3} \chi-k_{t} U_{\infty}^{2} t, \\
A_{t}^{*} & =A_{t}-B \\
\epsilon g(\phi, \psi) & =\int_{-\infty}^{\phi}\left(\frac{1}{U_{0}^{2}(\zeta, \psi)}-1\right) d \zeta .
\end{aligned}
$$

The function $\epsilon g(\phi, \psi)$ is Lighthill's drift function (Lighthill, 1956) in $(\phi, \psi)$-space, which represents the distortion of fluid material lines relative to (non-dimensionalised) uniform convection at speed 1 .

We substitute the solution for $\boldsymbol{v}$ into (1.1.2.2) to find the governing equation for gustaerofoil interaction. Neglecting terms of $O(\epsilon)$, Kerschen \& Myers (1987) show that the modified potential, $h(\phi, \psi)$ for the unsteady disturbance satisfies the non-dimensionalised equation

$$
\begin{array}{r}
\frac{\partial^{2} h}{\partial \phi^{2}}+\frac{\partial^{2} h}{\partial \psi^{2}}+k^{2} w^{2}\left(1-2 \beta_{\infty}^{2} \epsilon q\right) h+\frac{(\gamma+1) M_{\infty}^{4} \epsilon q}{\beta_{\infty}^{2}}\left(\frac{\partial^{2} h}{\partial \psi^{2}}+2 i k \delta \frac{\partial h}{\partial \phi}+k^{2}\left(w^{2}+\delta^{2}\right) h\right) \\
-\frac{(\gamma+1) M_{\infty}^{4} \epsilon}{\beta_{\infty}^{2}} \frac{\partial q}{\partial \phi}\left(\frac{\partial h}{\partial \phi}-\mathrm{i} k \delta h\right)=k \epsilon S(\phi, \psi) \mathrm{e}^{\mathrm{i} k \Omega},
\end{array}
$$

where

$$
\delta=k_{t} / \beta_{\infty}^{2}, \quad w^{2}=\left(M_{\infty} \delta\right)^{2}-\left(k_{3} / \beta_{\infty}\right)^{2}, \quad \Omega=\delta \phi+k_{n} \psi+\epsilon g(\phi, \psi)
$$

and

$S(\phi, \psi)=\frac{2}{\beta_{\infty}^{2}}\left(\mathrm{i}\left(A_{t}^{*}-A_{n} k_{n} \beta_{\infty}^{3}\right) q+\mathrm{i}\left(\beta_{\infty}^{2} k_{n} A_{t}^{*}+A_{n} \beta_{\infty}\right) \mu+\frac{A_{t}^{*} M_{\infty}^{2}}{k} \frac{\partial q}{\partial \phi}+\frac{A_{n} M_{\infty}^{2} \beta_{\infty}}{k} \frac{\partial q}{\partial \psi}\right)$.

The drift function, $g(\phi, \psi)$, reduces to

$$
g(\phi, \psi)=-2 \int_{-\infty}^{\phi} q(\eta, \psi) d \eta
$$

and the boundary condition is

$$
\frac{\partial h}{\partial \psi}+\left.M_{\infty}^{2} \epsilon \frac{\partial q}{\partial \psi} h\right|_{\psi=0}=\left.\left(-\frac{A_{n}}{\beta_{\infty}}+2 \epsilon \mu A_{t}^{*}+\frac{A_{n} M_{\infty}^{2} \epsilon q}{\beta_{\infty}}\right) \mathrm{e}^{\mathrm{i} k \Omega}\right|_{\psi=0}
$$

Due to the gust inducing vortical perturbations in the flow, (2.1.0.5a) contains a 
source term, $S$, that was not present for the sound-aerofoil interaction case where no such vortical perturbation was introduced at infinity.

\subsection{Leading-Edge Inner Solution}

Here we move to inner variables, $(\Phi, \Psi)=k(\phi, \psi)$, as we did in Section 1.2. By using the inner limit of (1.1.1.2), given by (1.2.0.1), we can evaluate the velocity potential, $\epsilon F$, at the leading edge (imposing $F(0)=0$ again), and hence we find that

$$
g(R, \theta) \sim 2 F_{R}(-\infty)+O\left(k^{-1}\right)
$$

where $F_{R}(z)=\operatorname{Re}(F(z))$.

In the leading-edge region, the governing equation, (2.1.0.5a), becomes

$$
\begin{gathered}
\frac{\partial^{2} H}{\partial \Phi^{2}}+\frac{\partial^{2} H}{\partial \Psi^{2}}+w^{2}\left(1-2 \beta_{\infty}^{2} \epsilon q\right) H+\frac{(\gamma+1) M_{\infty}^{4}}{\beta_{\infty}^{2}} \epsilon q\left(\frac{\partial^{2} H}{\partial \Psi^{2}}+2 i \delta \frac{\partial H}{\partial \Phi}+\left(w^{2}+\delta^{2}\right) H\right) \\
-\frac{(\gamma+1) M_{\infty}^{4}}{\beta_{\infty}^{2}} \epsilon \frac{\partial q}{\partial \Phi}\left(\frac{\partial H}{\partial \Phi}-\mathrm{i} \delta H\right)=\frac{\epsilon}{k} \mathrm{e}^{\mathrm{i} \bar{\Omega}} S(\Phi, \Psi), \quad \text { (2.2.0.2a) } \\
\bar{\Omega}=\delta \Phi+k_{n} \Psi+k \epsilon g(\Phi, \Psi), \\
S(\Phi, \Psi)=\frac{2}{\beta_{\infty}^{2}}\left(\mathrm{i} q\left(A_{t}^{*}-A_{n} k_{n} \beta_{\infty}^{3}+\mathrm{i} \beta_{\infty} \mu\left(\beta_{\infty} k_{n} A_{t}^{*}+A_{n}\right)+A_{t}^{*} M_{\infty}^{2} \frac{\partial q}{\partial \Phi}+A_{n} M_{\infty}^{2} \beta_{\infty} \frac{\partial q}{\partial \Psi}\right),\right.
\end{gathered}
$$

subject to boundary condition

$$
\frac{\partial H}{\partial \Psi}+\left.M_{\infty}^{2} \epsilon \frac{\partial q}{\partial \Psi} H\right|_{\Psi=0 \pm}=\left.\frac{1}{k}\left(-\frac{A_{n}}{\beta_{\infty}}+2 \epsilon \mu A_{t}^{*}+\frac{A_{n} M_{\infty}^{2} \epsilon q}{\beta_{\infty}}\right) \mathrm{e}^{\mathrm{i} \mathrm{i} \Omega}\right|_{\psi=0}
$$

The inner solution can be expanded as

$$
H(\Phi, \Psi)=\frac{1}{k} \mathrm{e}^{2 \mathrm{i} k \epsilon F_{R}(-\infty)}\left(H_{0}+\epsilon t^{\prime} \sqrt{k}\left(H_{1}+H_{2}+H_{3}\right)+\epsilon \alpha_{\text {eff }}^{\prime} \sqrt{k}\left(P_{1}+P_{2}+P_{3}\right)+O(\epsilon)\right)
$$

which varies from the sound-aerofoil inner solution ansatz, (1.2.0.3), only by two factors; $\mathrm{e}^{2 \mathrm{i} k \epsilon F_{R}(-\infty)}$ which represents the drift of the gust due to the background flow slowing as it approaches the aerofoil, and $1 / k$ which is caused by the overall scaling of the incident perturbation chosen in this chapter compared to the previous chapter. Recall $t=\epsilon t^{\prime}$ and $\alpha_{\text {eff }}=\epsilon \alpha_{\text {eff }}^{\prime}$ represent the thickness and camber parameters of the aerofoil respectively. 


\subsubsection{Solution for the Flat-Plate Term $H_{0}$}

$H_{0}$ represents the blocking of the vertical gust velocity by the aerofoil, and it satisfies

$$
\begin{aligned}
D\left(H_{0}\right) & =0, \\
\left.\frac{\partial H_{0}}{\partial \Psi}\right|_{\substack{\Phi>0 \\
\Psi=0}} & =-\frac{A_{n}}{\beta_{\infty}} \mathrm{e}^{\mathrm{i} \delta \Phi},
\end{aligned}
$$

where $D$ is the Helmholtz operator defined in (1.2.1.2). We solve this using identical methods to the flat-plate solution for sound-aerofoil interaction, finding that

$$
H_{0}=-\frac{A_{n} \operatorname{sgn}(\Psi)}{\beta_{\infty} 2 \pi \sqrt{\delta+w}} \int_{-\infty}^{\infty} \frac{\mathrm{e}^{-\mathrm{i} \lambda \Phi-|\Psi| \sqrt{\lambda^{2}-w^{2}}}}{(\lambda+\delta) \sqrt{\lambda+w}} d \lambda
$$

This solution has outer limit

$$
\begin{aligned}
H_{0} & \sim L_{0}(\theta) \frac{\mathrm{e}^{\mathrm{i} k w r}}{\sqrt{k r}}+O\left(k^{-3 / 2}\right), \\
L_{0}(\theta) & =-\frac{A_{n} \mathrm{e}^{-\mathrm{i} \pi / 4} \cos \theta / 2}{\beta_{\infty} \sqrt{\pi} \sqrt{\delta+w}(\delta-w \cos \theta)},
\end{aligned}
$$

which is non-singular for all values of $\theta$. The gust does not generate Fresnel regions; mathematically this can be seen by comparing the Fresnel-generating term in soundaerofoil interaction from (1.2.1.4), $\cos \chi-\cos \theta$, to its corresponding term in (2.2.1.2), $\delta-w \cos \theta$. It is clear there are real $\theta$ solutions to $\cos \chi=\cos \theta$, however a real $\theta$ solution to $\delta=w \cos \theta$ could only occur if $M_{\infty} \geq 1$. We consider strictly subsonic flow, hence singularities of this type never occur. This flat-plate solution is discussed in detail in Myers (1987) and Tsai (1992).

\subsubsection{Solution for Thickness-Related Term $H_{1}$}

$H_{1}$ arises from the influence of aerofoil thickness on the surface boundary condition. Our asymptotic approximation of the drift function, (2.2.0.1), shows no local distortion of the gust in the vicinity of the aerofoil surface as it is constant on $\theta=0,2 \pi$. Hence in the local leading-edge region the effect of thickness on the boundary condition is seen as a change of direction of the unit normal. It satisfies

$$
D\left(H_{1}\right)=0
$$




$$
\left.\frac{\partial H_{1}}{\partial \Psi}\right|_{\substack{\Phi>0 \\ \Psi=0 \pm}}=\frac{2 a A_{t}^{*}}{\beta_{\infty} \sqrt{\Phi}} \operatorname{sgn}(\Psi) \mathrm{e}^{\mathrm{i} \delta \Phi}
$$

which has solution

$$
H_{1}=-\frac{\mathrm{e}^{\mathrm{i} \pi / 4} A_{t}^{*} a}{\sqrt{\pi} \beta_{\infty}} \int_{-\infty}^{\infty} \frac{\mathrm{e}^{-\mathrm{i} \lambda \Phi-|\Psi| \sqrt{\lambda^{2}-w^{2}}}}{\sqrt{\lambda^{2}-w^{2}} \sqrt{\lambda+\delta}} d \lambda
$$

with outer limit

$$
\begin{aligned}
H_{1} & \sim L_{1}(\theta) \frac{\mathrm{e}^{\mathrm{i} k w r}}{\sqrt{k r}}+O\left(k^{-3 / 2}\right), \\
L_{1}(\theta) & =-\frac{\mathrm{i} A_{t}^{*} a \sqrt{2}}{\beta_{\infty} \sqrt{w} \sqrt{\delta-w \cos \theta}} .
\end{aligned}
$$

This is the generalised form of the $H_{1}$ solution presented in Tsai (1992). Recall, $a$ is defined by the leading edge of the aerofoil where $y \sim 2 a t^{\prime} \sqrt{x}$.

\subsubsection{Solution for Thickness-Related Term $\mathrm{H}_{2}$}

$\mathrm{H}_{2}$ arises from the volume source terms in the local leading-edge region which come about due to the convected disturbance in the non-uniform flow around the nose. We see both variations in amplitude and phase of the vortical velocity contributing to the source terms. This is generated in a different way to the $H_{2}$ term for sound-aerofoil interaction because of the ability of the gust to generate a vortical source within the flow. $\mathrm{H}_{2}$ therefore satisfies

$$
\begin{aligned}
& D\left(H_{2}\right)=\mathrm{e}^{\mathrm{i} \delta \Phi+\mathrm{i} k_{n} \Psi}\left(\frac{C_{1} \cos , \theta / 2+C_{2} \sin \theta / 2}{\sqrt{R}}\right.\left.+\frac{C_{3} \cos 3 \theta / 2+C_{4} \sin 3 \theta / 2}{R^{3 / 2}}\right), \\
&\left.\frac{\partial H_{2}}{\partial \Phi}\right|_{\substack{\Phi>0 \\
\Psi=0}}=0 \\
& C_{1}=\frac{2 a \mathrm{i}}{\beta_{\infty}^{2}}\left(\beta_{\infty} k_{n} A_{t}^{*}+A_{n}\right), \\
& C_{2}=-\frac{2 a \mathrm{i}}{\beta_{\infty}^{3}}\left(A_{t}^{*}-k_{n} A_{n} \beta_{\infty}^{3}\right), \\
& C_{3}=-\frac{A_{n} M_{\infty}^{2} a}{\beta_{\infty}^{2}}, C_{4}=\frac{A_{t}^{*} M_{\infty}^{2} a}{\beta_{\infty}^{3}} .
\end{aligned}
$$

The solution for $H_{2}$ is separated into complementary and particular solutions, $H_{2 c}$ and $H_{2 p}$ respectively. To solve for the particular solution we take the Fourier transform of 
(2.2.3.1) with respect to both coordinates and invert accordingly. We leave the solution in terms of two integrals;

$$
\begin{aligned}
& H_{2 p}(\Phi, \Psi)= \\
& \frac{-\mathrm{e}^{\pi \mathrm{i} / 4}}{8 \sqrt{\pi}\left(\delta^{2}+k_{n}^{2}\right)} \int_{-\infty}^{\infty}\left(\frac{(\delta+\lambda) f_{1}(\lambda)+\mathrm{i} k_{n} f_{2}(\lambda)}{\sqrt{\lambda^{2}-w^{2}}}-\operatorname{sgn}(\Psi) f_{2}(\lambda)\right) \frac{e^{a(\lambda, \Phi, \Psi)} d \lambda}{\sqrt{\lambda+\delta}\left(\lambda-\lambda_{1}\right)\left(\lambda-\lambda_{2}\right)} \\
& \quad+\frac{\mathrm{e}^{\mathrm{i} k_{n} \Psi+\mathrm{i} \pi / 4}}{8 \sqrt{\pi}\left(\delta^{2}+k_{n}^{2}\right)} \int_{-\infty}^{\infty}\left(\frac{f_{1}(\lambda)}{\sqrt{\lambda+\delta^{\prime}}}-\frac{\operatorname{sgn}(\Psi) f_{2}(\lambda)}{\sqrt{\lambda+\delta}}\right) \frac{e^{h(\lambda, \Phi, \Psi)} d \lambda}{\left(\lambda-\lambda_{1}\right)\left(\lambda-\lambda_{2}\right)}, \quad \text { (2.2.3.3a) }
\end{aligned}
$$

where

$$
\begin{aligned}
f_{1}(\lambda) & =\left(\mathrm{i} C_{2}-2 C_{4}(\lambda+\delta)\right)\left(k_{n}^{2}-\delta^{2}-w^{2}-2 \delta \lambda\right)+2 \mathrm{i} k_{n}(\lambda+\delta)\left(C_{1}+2 \mathrm{i} C_{3}(\lambda+\delta)\right), \\
f_{2}(\lambda) & =2 \mathrm{i} k_{n}\left(\lambda+\delta\left(\mathrm{i} C_{2}-2 C_{4}(\lambda+\delta)\right)+\left(C_{1}+2 \mathrm{i} C_{3}(\lambda+\delta)\right)\left(k_{n}^{2}-\delta^{2}-w^{2}-2 \delta \lambda\right),\right. \\
\lambda_{1,2} & =-\frac{\delta}{2}\left(\frac{\delta^{2}+k_{n}^{2}+w^{2}}{\delta^{2}+k_{n}^{2}}\right) \pm \frac{\mathrm{i} k_{n}}{2}\left(\frac{\delta^{2}+k_{n}^{2}-w^{2}}{\delta^{2}+k_{n}^{2}}\right) .
\end{aligned}
$$

The acoustic phase is

$$
a(\lambda, \Phi, \Psi)=-\mathrm{i} \lambda \Phi-|\Psi| \sqrt{\lambda^{2}-w^{2}}
$$

and the hydrodynamic phase is

$$
h(\lambda, \Phi, \Psi)=-\mathrm{i} \lambda \Phi-|\Psi| \sqrt{(\lambda+\delta)\left(\lambda+\delta^{\prime}\right)}
$$

Here we assume $\delta$ has a small positive imaginary part, and $\delta^{\prime}$ is the corresponding $\delta$ but with a small negative imaginary part, to ensure convergence of the relevant integrals. These imaginary parts are set to zero at the end of the analysis.

The complementary solution is solved using the Wiener-Hopf method to give

$$
\begin{aligned}
H_{2 c}(\Phi, \Psi)=\frac{-\operatorname{sgn}(\Psi) \mathrm{e}^{\mathrm{i} \pi / 4}}{8 \sqrt{\pi}\left(\delta^{2}+k_{n}^{2}\right)} \int_{-\infty}^{\infty}\left\{4 \mathrm{i}\left(\delta C_{3}+k_{n} C_{4}\right)\right. \\
\quad+\left(\frac{\sqrt{\lambda+w} f_{2}(\lambda)}{\sqrt{\lambda+\delta}\left(\lambda-\lambda_{2}\right)}-\frac{\sqrt{\lambda_{1}+w} f_{2}\left(\lambda_{1}\right)}{\sqrt{\lambda_{1}+\delta}\left(\lambda_{1}-\lambda_{2}\right)}\right) \frac{1}{\lambda-\lambda_{1}} \\
\left.\quad+\frac{\left(\lambda_{2}+\delta\right) f_{2}\left(\lambda_{2}\right)+\mathrm{i} k_{n} f_{1}\left(\lambda_{2}\right)}{\sqrt{\lambda_{2}-w} \sqrt{\lambda_{2}+\delta^{\prime}}\left(\lambda_{2}-\lambda\right)\left(\lambda-\lambda_{1}\right)}\right\} \frac{\mathrm{e}^{a(\lambda, \Phi, \Psi)}}{\sqrt{\lambda+w}} d \lambda .
\end{aligned}
$$

Expanding $\mathrm{H}_{2}$ in terms of outer coordinates and using the method of steepest descent 
gives

$$
H_{2} \sim \frac{\mathrm{e}^{\mathrm{i} k\left(\delta \phi+k_{n} \psi\right)}}{\sqrt{k r}}\left(\frac{C_{1} \cos \theta / 2+C_{2} \sin \theta / 2}{\delta^{2}+k_{n}^{2}-w^{2}}\right)+L_{2}(\theta) \frac{\mathrm{e}^{\mathrm{i} k w r}}{\sqrt{k r}}+O\left(k^{-3 / 2}\right),
$$

where

$$
\begin{array}{r}
L_{2}(\theta)=\frac{-\mathrm{i}\left[(\delta-w \cos \theta) f_{1}(-w \cos \theta)+\mathrm{i} k_{n} f_{2}(-w \cos \theta)\right]}{4 \sqrt{2 w}\left(\delta^{2}+k_{n}^{2}\right) \sqrt{\delta-w \cos \theta}\left(\lambda_{1}+w \cos \theta\right)\left(\lambda_{2}+w \cos \theta\right)} \\
-\frac{\cos \theta / 2}{4\left(\lambda_{1}-\lambda_{2}\right)\left(\delta^{2}+k_{n}^{2}\right)}\left(4\left(\lambda_{1}-\lambda_{2}\right)\left(\mathrm{i} \delta C_{3}+\mathrm{i} k_{n} C_{4}\right)+\frac{\sqrt{\lambda_{1}+w} f_{2}\left(\lambda_{1}\right)}{\sqrt{\lambda_{1}+\delta}\left(\lambda_{1}+x \cos \theta\right)}\right. \\
\left.\quad-\frac{\left(\lambda_{2}+\delta\right) f_{2}\left(\lambda_{2}\right)+\mathrm{i} k_{n} f_{1}\left(\lambda_{2}\right)}{\left(\lambda_{2}+w \cos \theta\right) \sqrt{\lambda_{2}-w} \sqrt{\lambda_{2}+\delta^{\prime}}}\right)
\end{array}
$$

Notice that the integral in (2.2.3.3) containing the hydrodynamic phase does not have a point of stationary phase, so its contribution is obtained by deforming the contour of integration onto two rays along which the integral endpoints dominate for $k r \gg 1$ (see Myers (1987) for full details). The first term in the above expansion, (2.2.3.4a), is due to this hydrodynamic phase and can be shown to match with a hydrodynamic particular solution in the outer region which also describes hydrodynamic motion (in Section 2.3). The second term is the sound generated by the volume sources and the scattering of this sound by the aerofoil leading edge, to leading order. These solutions are the generalised form to those presented in Tsai (1992).

\subsubsection{Solution for Thickness-Related Term $H_{3}$}

$\mathrm{H}_{3}$ is the solution arising from the interaction of the scattered sound at leading order, (2.2.1.2), with the non-uniform flow around the aerofoil nose. It satisfies

$$
\begin{gathered}
D\left(H_{3}\right)=-\frac{2 w^{2} a \beta_{\infty} \sin \theta / 2}{\sqrt{R}} H_{0}+\frac{(\gamma+1) M_{\infty}^{4} a \sin \theta / 2}{\beta_{\infty}^{3} \sqrt{R}}\left(\frac{\partial^{2} H_{0}}{\partial \Psi^{2}}+2 \mathrm{i} \delta \frac{\partial H_{0}}{\partial \Phi}+\left(w^{2}+\delta^{2}\right) H_{0}\right) \\
+\frac{a(\gamma+1) M_{\infty}^{4} \sin 3 \theta / 2}{2 \beta_{\infty}^{3} R^{3 / 2}}\left(\frac{\partial H_{0}}{\partial \Phi}-\mathrm{i} \delta H_{0}\right), \quad \text { (2.2.4.1a) } \\
\left.\frac{\partial H_{3}}{\partial \Psi}\right|_{\substack{\Phi>0 \\
\Psi=0}}=\frac{M_{\infty}^{2} a H_{0} \operatorname{sgn}(\Psi)}{2 \beta_{\infty} R^{3 / 2}} .
\end{gathered}
$$

This is solved similarly to the $H_{3}$ term in the sound-aerofoil interaction problem of Section 1.2.4. The problem is once again separated into a particular solution, $H_{3 p}$, and complementary solutions $H_{3 c_{1}}+H_{3 c_{2}}+H_{3 c_{3}}$. Details of the solution can be found in 
Appendix A (including corrections to Tsai's results), along with the outer limits of the solutions;

$H_{3 p} \sim-\frac{A_{n} a \mathrm{e}^{\mathrm{i} \pi / 4} w \sin \theta \mathrm{e}^{\mathrm{i} w k r}}{\sqrt{\pi} \sqrt{\delta+w}(\delta-w \cos \theta)}\left(1-\frac{(\gamma+1) M_{\infty}^{4}}{2 \beta_{\infty}^{4} w^{2}}(\delta-w \cos \theta)^{2}\right)+L_{3 p}(\theta) \frac{\mathrm{e}^{\mathrm{i} w k r}}{\sqrt{k r}}+O(1 / k)$

and

$$
H_{3 c_{i}} \sim L_{3 c_{i}}(\theta) \frac{\mathrm{e}^{\mathrm{i} w k r}}{\sqrt{k r}}+O\left(k^{-3 / 2}\right) \quad \text { for } i=1,2,3
$$

\subsubsection{Solution for Camber-Related Terms $P_{i}, i=1,2,3$}

Myers \& Kerschen (1997) provide leading-edge inner solutions for a flat plate with effective angle of attack $\alpha_{\text {eff. }}$ Since camber and thickness are essentially independent effects in the leading-edge inner region we can immediately use their results, which yield outer limits

$$
P_{i}(r, \theta) \sim D_{i}(\theta) \frac{\mathrm{e}^{\mathrm{i} k w r}}{\sqrt{k r}}
$$

where

$$
\begin{aligned}
D_{1}(\theta)= & \frac{2 \mathrm{i} A_{n} \delta}{\sqrt{w}(\delta-w \cos \theta)^{3 / 2}}, \\
D_{2}(\theta)= & \tilde{L}_{2}(\theta), \\
D_{3}(\theta)= & \frac{\mathrm{i} A_{n}}{\sqrt{w(\delta-w \cos \theta)}}\left(1-\frac{M_{\infty}^{2}}{\beta_{\infty}^{2}}-\frac{\delta}{\delta-w \cos \theta}\right) \\
& +\frac{\mathrm{i} A_{n}(\gamma+1) M_{\infty}^{4}}{w^{3 / 2} \sqrt{\delta+w} \beta_{\infty}^{4}}\left(\frac{\delta}{2} \cos \theta-\frac{w}{4} \cos 2 \theta\right) .
\end{aligned}
$$

$\tilde{L}_{2}(\theta)$ is related to $L_{2}(\theta)$ by changing the functions $(2.2 .3 .2)$ to

$$
\begin{aligned}
C_{1}^{\prime} & =\mathrm{i} 2^{3 / 2}\left(\frac{\delta A_{t}^{*}}{\beta_{\infty}}-k_{n} A_{n}\right), & C_{2}^{\prime} & =\mathrm{i} 2^{3 / 2}\left(\frac{k_{n} A_{t}^{*}}{\beta_{\infty}}+\delta A_{n}\right), \\
C_{3}^{\prime} & =-\frac{\sqrt{2} A_{t}^{*} M_{\infty}^{2}}{\beta_{\infty}^{3}}, & C_{4}^{\prime} & =-\frac{\sqrt{2} A_{n} M_{\infty}^{2}}{\beta_{\infty}^{2}} .
\end{aligned}
$$

\subsection{Outer Solutions}

We separate the solution into four parts, as done by Tsai (1992).

$$
h=h_{p}+h_{c}+h_{l}+h_{t}
$$


Here, $h_{p}$ is the particular solution due to the volume source that was not present during the sound-aerofoil interaction. It does not satisfy the boundary condition on the aerofoil surface hence we have $h_{c}$, a complementary solution, to rectify this. $h_{l}$ is the leadingedge acoustic outer solution (similarly $h_{t}$ is the trailing-edge acoustic outer solution) and these account for the propagation into the outer region of acoustic waves generated at the leading and trailing edges of the aerofoil respectively. To leading order, $h_{p}$ satisfies

$$
\frac{\partial^{2} h}{\partial \phi^{2}}+\frac{\partial^{2} h}{\partial \psi^{2}}+k^{2} w^{2} h=k \epsilon S(\phi, \psi) \mathrm{e}^{\mathrm{i} k \Omega(\phi, \psi)}+O(\epsilon)
$$

We want a solution with rapidly varying phase to match the source term, hence consider

$$
h_{p}=A(\phi, \psi) \mathrm{e}^{\mathrm{i} k \Omega(\phi, \psi)}
$$

and proceed as outlined by Tsai (1992) and Myers (1987). Solving for $A$ gives

$$
h_{p}=\frac{\epsilon}{k} \frac{S(\phi, \psi) \mathrm{e}^{\mathrm{i} k \Omega(\phi, \psi)}}{w^{2}-\left(\frac{\partial \Omega}{\partial \phi}\right)^{2}-\left(\frac{\partial \Omega}{\partial \psi}\right)^{2}}+O\left(\epsilon^{3}\right)
$$

The complementary solution must now satisfy the boundary condition on $\psi=0$

$$
\frac{\partial h_{c}}{\partial \psi}+M_{\infty}^{2} \epsilon \frac{\partial q}{\partial \psi} h_{c}=\left(-\frac{A_{n}}{\beta_{\infty}}+2 \epsilon \mu(\phi, 0) A_{t}^{*}+\frac{A_{n} \epsilon q(\phi, 0) M_{\infty}^{2}}{\beta_{\infty}}-\frac{\mathrm{i} k_{n} \epsilon S(\phi, 0)}{w^{2}-\delta^{2}-k_{n}^{2}}\right) \mathrm{e}^{\mathrm{i} k \Omega(\phi, 0)}
$$

Notice that the complementary solution also depends on the rapidly varying phase $e^{i k \Omega(\phi, 0)}$. We anticipate the complementary solution to decay exponentially with distance from the boundary, so we expect the solution to be exponentially small outside of a boundary layer region where $\psi=O(1 / k)$. This scaling can be seen by balancing terms in (2.3.0.4) for $\psi=O\left(k^{\nu}\right)$ and solving for $\nu$. The right hand side has terms of $O(1, t)$ hence for $h=O\left(\mathrm{e}^{\mathrm{i} k \Omega(\phi, 0)}\right)$ we find $\nu=-1$ satisfies. The physical interpretation of this is that the phase of this solution corresponds to convection by the oncoming gust of the knowledge of the boundary condition. Hence we set

$$
h_{c}=f(y, \phi) \mathrm{e}^{\mathrm{i} k \Omega(\phi, 0)}, \quad y=k|\psi|=O(1)
$$

and require

$$
\left.k \frac{\partial f}{\partial y}\right|_{y=0 \pm}=-\frac{A_{n}}{\beta_{\infty}}+2 \epsilon \mu(\phi, 0) A_{t}^{*}+\frac{A_{n} M_{\infty}^{2} \epsilon q(\phi, 0)}{\beta_{\infty}}-\frac{\mathrm{i} k_{n} \epsilon S(\phi, 0)}{w^{2}-\delta^{2}-k_{n}^{2}}
$$


hence

$$
h_{c}=\frac{\operatorname{sgn}(\psi)}{k m(\phi)}\left(-\frac{A_{n}}{\beta_{\infty}}+2 \epsilon \mu(\phi, 0) A_{t}^{*}+\frac{A_{n} M_{\infty}^{2} \epsilon q(\phi, 0)}{\beta_{\infty}}-\frac{\mathrm{i} k_{n} \epsilon S(\phi, 0)}{w^{2}-\delta^{2}-k_{n}^{2}}\right) \mathrm{e}^{-k|\psi| m(\phi)+\mathrm{i} k \Omega(\phi, 0)},
$$

for

$$
[m(\phi)]^{2}=\left(\frac{\partial \Omega(\phi, 0)}{\partial \phi}\right)^{2}-w^{2}\left(1-2 \beta_{\infty}^{2} \epsilon q(\phi, 0)\right) .
$$

This is as found in Myers (1987). We notice that both $h_{p}$ and $h_{c}$ convect with the mean flow, and hence are not acoustic waves, thus all the sound generated in this problem is from the local leading- and trailing-edge regions. The only sound in the outer region is that propagating away from these local regions. We expect therefore to find $h_{l}$ and $h_{t}$ to be of a geometric acoustic form, i.e. the phase of these solutions vary rapidly compared to the amplitude and the acoustic fields can be described as rays emanating from the leading and trailing edges. The hydrodynamic particular solution, $h_{p}$ matches to the leading-edge inner hydrodynamic solutions; details can be found in Myers \& Kerschen (1997) and Tsai (1992). The hydrodynamic complementary solution, $h_{c}$ matches to an inner trailing-edge hydrodynamic term.

\subsubsection{Leading-Edge Acoustic Outer Solution}

We solve similarly to the acoustic outer solution found in Section 1.3.1. We find that the outer solution is given by (1.3.1.1), where the $K_{i}$ are redefined appropriately with respect to the directivity functions, $L_{i}$ given in this chapter. This gives a leading-edge outer solution of

$$
h_{l}=\frac{\mathcal{D}_{l}(\theta)}{k^{3 / 2} \sqrt{r}} \mathrm{e}^{\mathrm{i} k w r+\mathrm{i} k w \epsilon V(\theta) \int_{0}^{r} q\left(r^{\prime}, \theta\right) d r^{\prime}+2 \mathrm{i} k \epsilon F_{R}(-\infty)},
$$

where

$$
\begin{aligned}
\mathcal{D}_{l}(\theta)=L_{0}(\theta) & +t \sqrt{k}\left(L_{1}(\theta)+L_{2}(\theta)+L_{3 p}(\theta)+L_{3 c_{1}}(\theta)+L_{3 c_{2}}(\theta)+L_{3 c_{3}}(\theta)\right) \\
& +\alpha_{\text {eff }} \sqrt{k}\left(D_{1}(\theta)+D_{2}(\theta)+D_{3}(\theta)\right)
\end{aligned}
$$

For a uniformly-valid outer solution we must replace the non-uniformly valid term $L_{3 c_{2}}(\theta)(k r)^{-1 / 2}$ by its uniformly-valid counterpart $L_{3 c_{2}}^{u}(r, \theta)$. 


\subsubsection{Trailing-Edge Acoustic Outer Solution}

The trailing-edge acoustic outer solution also takes the same form as previously found in Section 1.4.1,

$$
h_{t}=\frac{\mathcal{D}_{t}(\theta)}{k^{2} \sqrt{r_{t}}} \mathrm{e}^{\mathrm{i} k w r_{t}+\mathrm{i} k w \epsilon V\left(\theta_{t}\right) \int_{0}^{r_{t}} q\left(r_{t}^{\prime}, \theta_{t}\right) d r_{t}^{\prime}}
$$

$\mathcal{D}_{t}$ is determined by matching to the trailing-edge inner solution which follows in Section 2.5 .

\subsection{Leading-Edge Transition Solution}

The leading-edge transition solution is solved for using the same method as in Section 1.3.2. We suppose the total leading-edge acoustic solution is given by

$$
h_{l}^{u}=h_{l}+h_{l s}^{(t)}+h_{l s}^{(c)},
$$

where $h_{l s}^{(t, c)}$ are the thickness- and camber-related leading-edge transition solutions that correct for the boundary condition on the surface of the aerofoil. The thickness-related solution takes the form

$$
h_{l s}^{(t)}(\phi, \eta)=\frac{t}{k} \mathcal{G}(\phi, \eta) \mathrm{e}^{\mathrm{i} k w \phi+\mathrm{i} k w \epsilon V(0) \int_{0}^{r} q\left(r^{\prime}, 0\right) d r^{\prime}+2 \mathrm{i} k \epsilon F_{R}(-\infty)}
$$

which we solve using the Laplace transform to give

$$
\begin{aligned}
\mathcal{G}(\phi, \eta)=\frac{\mathrm{i} A_{n} w}{\sqrt{2 w} \pi \sqrt{\delta+w}(\delta-w)}\left(1-\frac{(\gamma+1) M_{\infty}^{4}}{2 \beta_{\infty}^{4} w^{2}}(\delta-w)^{2}\right) \\
\left(\int_{0}^{2}\left[\frac{a}{\xi}+\cdot \frac{\beta_{\infty}}{t^{\prime} \xi^{3 / 2}} \int_{0}^{\xi} \frac{\partial q^{(t)}}{\partial \theta}\left(r^{\prime}, 0\right) d r^{\prime}\right] \frac{\mathrm{e}^{\mathrm{i} \eta^{2} w / 2(\phi-\xi)}}{\sqrt{\phi-\xi}} d \xi\right. \\
\left.\quad+\int_{2}^{\phi}\left[\frac{a}{\xi}+\frac{\beta_{\infty}}{t^{\prime} \xi^{3 / 2}} \int_{0}^{2} \frac{\partial q^{(t)}}{\partial \theta}\left(r^{\prime}, 0\right) d r^{\prime}\right] \frac{\mathrm{e}^{\mathrm{i} \eta^{2} w / 2(\phi-\xi)}}{\sqrt{\phi-\xi}} d \xi\right)
\end{aligned}
$$

for $\phi>2$. This yields the generalised transition solution to Tsai's result which was the specialised case of a symmetric Joukowski aerofoil. Our new solution is more complicated than Tsai's because for an aerofoil of arbitrary shape, the complex potential cannot be specified from the beginning. For the symmetric Joukowski aerofoil, not only does one know $F$ from the start of the analysis, but $F$ can be written in a simple closed form. The camber-related transition solution can be obtained directly from Myers \& Kerschen 
(1997) since they present their solution in terms of the generalised camber distribution on the aerofoil, $\epsilon y^{(c)}(x)$. Hence the large (positive) $\phi$ limit of $h_{l s}^{(c)}$ is given by

$$
\begin{aligned}
h_{l s}^{(c)} \sim & \frac{\operatorname{sgn}(\psi) \mathrm{e}^{\mathrm{i} k w \sigma_{l}(\phi, 0)}}{k \sqrt{r}} \frac{\mathrm{e}^{3 \mathrm{i} \pi / 4} \epsilon V(0) P_{l}(0 \pm)}{\sqrt{2 \pi w} \beta_{\infty}}\left[\int_{0}^{2} \frac{\mathrm{e}^{\mathrm{i} w \eta^{2} / 2(\phi-\xi)}\left(\xi y^{(c) \prime}(\xi)-y^{(c)}(\xi)\right)}{\sqrt{\phi-\xi} \xi^{3 / 2}} d \xi\right. \\
& \left.+2^{3 / 2} y^{(c) \prime}(2)\left(\frac{\mathrm{e}^{\mathrm{i} w \eta^{2} / 2(\phi-2)} \sqrt{\phi-2}}{\phi}-\frac{|\eta| \mathrm{e}^{-\pi \mathrm{i} / 4} \sqrt{\pi w} \mathrm{e}^{\mathrm{i} w \eta^{2} / 2 \phi}}{\phi^{3 / 2}} \operatorname{erfc}\left[\frac{\mathrm{e}^{-\pi \mathrm{i} / 4} \sqrt{w}|\eta|}{\sqrt{\phi(\phi-2)}}\right]\right)\right], \\
= & \frac{1}{k} D_{l t r}(\theta) \frac{\mathrm{e}^{\mathrm{i} k w \sigma_{l}(r, \theta)}}{\sqrt{r}},
\end{aligned}
$$

with

$$
P_{l}(\theta)=L_{0}(\theta)+\alpha_{\mathrm{eff}} \sqrt{k}\left(D_{1}(\theta)+D_{2}(\theta)+D_{3}(\theta)\right),
$$

as given in Myers \& Kerschen (1997). We take the outer limits of the thickness dependent solution also to give the total far-field solution emanating from the leading edge:

$$
\begin{aligned}
h_{l}^{u}(r, \theta) \sim \frac{\mathrm{e}^{\mathrm{i} k w \sigma_{l}(r, \theta)}}{k^{3 / 2} \sqrt{r}}\left\{L_{0}(\theta)+t \sqrt{k}\left[L_{1}(\theta)+L_{2}(\theta)+L_{3 p}(\theta)+L_{3 c_{1}}(\theta)+L_{3 c_{2}}(\theta)+L_{3 c_{3}}(\theta)\right.\right. \\
+\frac{\operatorname{sgn}(\psi) A_{n} \mathrm{i} \sqrt{w}}{\sqrt{2} \pi \sqrt{\delta+w}(\delta-w)}\left(1-\frac{(\gamma+1) M_{\infty}^{4}}{2 \beta_{\infty}^{4} w^{2}}(\delta-w)^{2}\right) \\
\quad\left(\int_{0}^{2}\left[\frac{a}{\tau}+\frac{\beta_{\infty}}{t^{\prime} \tau^{3 / 2}} \int_{0}^{\tau} \frac{\partial q^{(t)}}{\partial \theta}\left(r^{\prime}, 0\right) d r^{\prime}\right] \mathrm{e}^{\mathrm{i} w k(1-\cos \theta) \tau} d \tau\right. \\
\left.\left.+\int_{2}^{\infty}\left[\frac{a}{\tau}+\frac{\beta_{\infty}}{t^{\prime} \tau^{3 / 2}} \int_{0}^{2} \frac{\partial q^{(t)}}{\partial \theta}\left(r^{\prime}, 0\right) d r^{\prime}\right] \mathrm{e}^{\mathrm{i} w k(1-\cos \theta) \tau} d \tau\right)\right] \\
\left.\quad+\alpha_{\mathrm{eff}} \sqrt{k}\left[D_{1}(\theta)+D_{2}(\theta)+D_{3}(\theta)\right]+\sqrt{k} D_{l t r}(\theta)\right\}, \quad \text { 2.4.0.7a) }
\end{aligned}
$$

which we write as

$$
h_{l}^{u} \sim \frac{\mathrm{e}^{\mathrm{i} k w \sigma_{l}(r, \theta)}}{k^{3 / 2} \sqrt{r}} D_{l}(\theta) .
$$

We have only given explicit results for $h_{l s}^{(t, c)}$ in the region $\phi>2$ because these are used to find the pressure jump across the wake. In the far field, except for $\theta \sim 0,2 \pi$, the transition solutions are negligible. The transition solutions in the region $0<\phi<2$ are equivalent to those found in Chapter 1, Section 1.3.2, and in Myers \& Kerschen (1997). 


\subsection{Trailing-Edge Inner Solution}

Trailing-edge inner coordinates are defined as $\left(\Phi_{t}, \Psi_{t}\right)=k\left(\phi_{t}, \psi_{t}\right)$ such that

$$
\phi=2 \pm \frac{\Gamma}{2}+\alpha_{t}+\phi_{t}, \quad \psi=\psi_{t}
$$

where $\Gamma$ is the circulation around the aerofoil given by (1.4.1.2), and $\alpha_{t}$ is given by (1.4.1.4). Let $H_{t}\left(\Phi_{t}, \Psi_{t}\right)$ be the inner solution around the trailing edge, then upon substituting the inner trailing-edge coordinates into (2.1.0.5a) we find,

$$
\frac{\partial^{2} H_{t}}{\partial \Phi_{t}^{2}}+\frac{\partial^{2} H_{t}}{\partial \Psi_{t}^{2}}+w^{2} H_{t}=\frac{\epsilon S(\phi, \psi)}{k} \mathrm{e}^{\mathrm{i} k \Omega(\phi, \psi)} .
$$

In the leading-edge region, we found that $q=O\left(k^{1 / 2}\right)$ in (1.2.0.1), however in the trailingedge region $q=O(1)$, so we can neglect this source term in the governing equation as it is $O\left(\epsilon^{2}\right)$. Hence $H_{t}$ satisfies the Helmholtz equation subject to boundary condition

$$
\left.\frac{\partial H_{t}}{\partial \Psi_{t}}\right|_{\substack{\Phi_{t}<0 \\ \Psi_{t}=0}}=-\frac{A_{n}}{k \beta_{\infty}} \mathrm{e}^{\mathrm{i} \delta \Phi_{t}+\mathrm{i} k \delta\left(2 \pm \Gamma / 2+\alpha_{t}\right)+\mathrm{i} k \epsilon g(\phi, 0)}+O\left(\epsilon^{5 / 2}\right),
$$

where $g(\phi, 0) \sim 2 F_{R}(-\infty)$ is given by using (2.2.0.1). Pressure and normal velocity must be continuous across the wake of the aerofoil, $\Psi_{t}=0, \Phi_{t}>0$, which arise from the unsteady Kutta condition, requiring that the wake found behind the aerofoil can be thought of as a vortex sheet between the solutions of the upper and lower surfaces of the aerofoil that leaves the trailing edge of the aerofoil smoothly.

Myers (1987) gives a solution satisfying the Helmholtz equation and normal velocity condition;

$$
H_{t}^{h}=\frac{\operatorname{sgn}\left(\Psi_{t}\right) A_{n}}{\beta_{\infty} \sqrt{\delta^{2}-w^{2}}} \mathrm{e}^{\mathrm{i} k \delta\left(2 \pm \Gamma / 2+\alpha_{t}\right)+\mathrm{i} k \epsilon g(2,0 \pm)} \mathrm{e}^{-\left|\Psi_{t}\right| \sqrt{\delta^{2}-w^{2}}} \mathrm{e}^{\mathrm{i} \delta \Phi_{t}}
$$

This is a hydrodynamic solution rather than an acoustic solution with outer limit matching onto the hydrodynamic complementary solution, $h_{c}$ mentioned in Section 2.3. It is a disturbance convected at the free stream speed; Tsai (1992) confirms this, and reminds us that this does not contradict trailing-edge noise theories (Howe, 1978) since here we have neglected the presence of a turbulent boundary layer. There are, however, pressure fluctuations across the wake generated due to the scattered leading-edge acoustic ray field as it extends to the outer region. To satisfy the required pressure condition we therefore need a local inner acoustic solution around the trailing edge that cancels 
this pressure jump. Further, we require a transition solution to match the trailing-edge solution onto the outer solution that satisfies the required boundary conditions. Recall the non-dimensionalised modified pressure at the trailing edge due to the leading-edge ray field is given by

$$
p_{l}=\mathrm{i} k(\delta-w \cos \theta) h_{l s}(r, \theta) \mathrm{e}^{-\mathrm{i} k \delta M_{\infty}^{2} \phi} .
$$

The pressure jump across the wake is given in Appendix B, where we define $\Delta p\left(\phi_{t}\right)$ for gust-aerofoil interaction. As in Chapter 1, we separate this pressure jump into contributions from thickness and camber. This problem (which due to the hydrodynamic solution requires zero velocity on either side of the wake) now forms a similar set of equations as the sound-aerofoil interaction problem (but with an alternative definition for $\Delta p$ ) given in Section 1.4.2, hence we can immediately write down the inner solution as

$$
H_{t}=\frac{\operatorname{sgn}\left(\Psi_{t}\right) \mathrm{i} \sqrt{2 w} \Delta p(0) \mathrm{e}^{\mathrm{i} C_{ \pm}}}{4 \pi k^{3 / 2}} \int_{-\infty}^{\infty} \frac{\mathrm{e}^{-\mathrm{i} \lambda \Phi_{t}-\left|\Psi_{t}\right| \sqrt{\lambda^{2}-w^{2}}}}{(\lambda+\delta)(\lambda+w) \sqrt{\lambda-w}} d \lambda
$$

This has outer limit

$$
H_{t} \sim T\left(\theta_{t}\right) \frac{\mathrm{e}^{\mathrm{i} k w r_{t}}}{k^{2} \sqrt{r_{t}}}+O\left(k^{-3}\right)
$$

where

$$
T\left(\theta_{t}\right)=-\frac{\mathrm{e}^{\mathrm{i} \pi / 4+\mathrm{i} C_{ \pm}} \Delta p(0) \operatorname{sgn}\left(\psi_{t}\right)}{2 \sqrt{\pi w\left(1-\cos \theta_{t}\right)}\left(\delta-w \cos \theta_{t}\right)},
$$

The uniformly-valid outer limit of $H_{t}$ is obtained by replacing $T$ with

$$
\begin{array}{r}
T^{u}\left(r_{t}, \theta_{t}\right)=\frac{\operatorname{sgn}\left(\psi_{t}\right) \mathrm{i} \Delta p(0) \mathrm{e}^{\mathrm{i} C_{ \pm}}}{2(\delta-w)}\left[\operatorname{erfc}\left(\mathrm{e}^{-\mathrm{i} \pi / 4} \sqrt{w\left(1-\cos \theta_{t}\right) k r_{t}}\right) \mathrm{e}^{\mathrm{i} k w r_{t}\left(\cos \theta_{t}-1\right)} \sqrt{k r_{t}}\right. \\
\left.-\frac{\sqrt{2 w} \mathrm{e}^{\mathrm{i} \pi / 4}\left|\sin \theta_{t} / 2\right|}{\sqrt{\pi}\left(\delta-w \cos \theta_{t}\right)}\right]
\end{array}
$$

Similarly to the sound-aerofoil case, this trailing-edge inner solution matches the trailingedge outer acoustic solution, (2.3.2.1), if $\mathcal{D}_{t}\left(\theta_{t}\right)=T\left(\theta_{t}\right)$.

\subsection{Trailing-Edge Transition Solution}

The trailing-edge inner solution derived in the previous section is similar to the solution found in the sound-aerofoil interaction, but with a redefinition of certain functions (for example $\Delta p$ ). It is also true that the leading-edge camber-related inner solution for gust-aerofoil interaction can be written in a similar form to that found in sound-aerofoil 
interaction. This fact allows us to use the solution derived in Section 1.4.3 here. It also immediately tells us that we have a matching between our trailing-edge transition solution and our trailing-edge inner solution.

\subsection{Total Far-Field Solution}

The common terms in our trailing-edge transition solution and our trailing-edge acoustic outer solution are as given in Section 1.4.5. Subtracting these from the sum of the trailing-edge acoustic outer solution, and the trailing-edge transition solution yields $h_{t}^{u}$, which describes the propagation into the outer region of the sound generated at the trailing edge of the aerofoil. The total acoustic field emanating from the trailing edge of the aerofoil, $h_{t}^{u}$, is given by

$$
\begin{aligned}
& h_{t}^{u}\left(r_{t}, \theta_{t}\right) \sim \\
& \frac{\mathrm{e}^{\mathrm{i} C_{ \pm}} \mathrm{e}^{\mathrm{i} k w \sigma_{t}\left(r_{t}, \theta_{t}\right)}}{\sqrt{2} \sqrt{r_{t}} k^{2}(\delta-w \cos \theta)}\left\{-\operatorname{sgn}\left(\psi_{t}\right) \mathrm{ie}^{-2 \mathrm{i} k w\left(1-\cos \theta_{t}\right)}\left(P_{+}-P_{-}\right) \operatorname{erfc}\left[\mathrm{e}^{-\pi \mathrm{i} / 4} \sqrt{2 k w(1-\cos \theta)}\right]\right. \\
& -\frac{2 \sqrt{k} w V(0)\left(\alpha_{i}-\epsilon y^{(c) \prime}(2)\right)\left(P_{+}-P_{-}\right) \mathrm{e}^{\pi \mathrm{i} / 4}}{\sqrt{\pi w} \beta_{\infty}}\left(1-\sqrt{2 k w \pi(1-\cos \theta)} \mathrm{e}^{-\pi \mathrm{i} / 4} \mathrm{e}^{-2 \mathrm{i} k w(1-\cos \theta)}\right. \\
& \left.\mathrm{x} \operatorname{erfc}\left[\mathrm{e}^{-\pi \mathrm{i} / 4} \sqrt{2 k w(1-\cos \theta)}\right]\right)+\frac{\operatorname{sgn}\left(\psi_{t}\right) \sqrt{k} w V(0)\left(P_{+}+P_{-}\right)}{\sqrt{2 w} \pi \beta_{\infty}} \sqrt{k w(1-\cos \theta)} \\
& \left.\mathrm{x} \int_{0}^{\infty} \mathrm{e}^{\mathrm{i} k w(1-\cos \theta) \xi} b_{t}(\xi) d \xi\right\}+\frac{\operatorname{sgn}(\psi) \mathrm{e}^{\mathrm{i} C_{ \pm}} \mathrm{e}^{\mathrm{i} k w \sigma_{t}\left(r_{t}, \theta_{t}\right)}}{\sqrt{r_{t}} k^{2}(\delta-w)}\left\{\frac{\Delta p^{(t)}(0) \mathrm{e}^{\pi \mathrm{i} / 4}\left|\sin \theta_{t} / 2\right|}{\sqrt{2 \pi w}(\delta-w \cos \theta)}\right. \\
& +\frac{\Delta p^{(t)}(0)}{\sqrt{2}} \sqrt{k} \mathrm{ie}^{-2 \mathrm{i} k w\left(1-\cos \theta_{t}\right)} \operatorname{erfc}\left[\mathrm{e}^{-\pi \mathrm{i} / 4} \sqrt{2 k w(1-\cos \theta)}\right] \\
& +\frac{t k A_{n} \mathrm{e}^{\pi i / 4} w}{2 \pi^{3 / 2} \sqrt{\delta+w}}\left(1-\frac{(\gamma+1) M_{\infty}^{4}}{2 \beta_{\infty}^{4} w^{2}}(\delta-w)^{2}\right) \mathrm{e}^{\mathrm{i} k\left(w-\delta M_{\infty}^{2}\right)\left(2+\alpha_{t}\right)+2 \mathrm{i} k \epsilon F_{R}(-\infty)} \\
& {\left[\mathrm { e } ^ { \mathrm { i } k ( w - \delta M _ { \infty } ^ { 2 } ) \Gamma / 2 + \mathrm { i } k w \epsilon \sigma _ { l 1 } ( 2 , 0 ) } \left\{\mathrm{e}^{\pi \mathrm{i} / 4} \frac{\sqrt{\pi}}{\sqrt{2 w}} \int_{0}^{2}\left(\frac{a}{\tau}+\frac{\beta_{\infty}}{t^{\prime} \tau^{3 / 2}} \int_{0}^{\tau} \frac{\partial q^{(t)}}{\partial \theta}(r, 0) d r\right) \mathrm{e}^{\mathrm{i} k w(1-\cos \theta) \tau}\right.\right.} \\
& \times \operatorname{erfc}\left[\mathrm{e}^{-\pi \mathrm{i} / 4} \sqrt{2-\tau} \sqrt{k w(1-\cos \theta)}\right] d \tau \\
& +2 a \sqrt{k(1-\cos \theta)} \int_{2}^{\infty} \mathrm{e}^{\mathrm{i} k w(1-\cos \theta) \xi^{2} / 2}\left[\log [2]-\log \left[\xi^{2} / 2\right]-\log \left[1-\sqrt{1-4 / \xi^{2}}\right]\right] d \xi \\
& +\frac{\beta_{\infty}}{t^{\prime}} \int_{0}^{2} \frac{\partial q^{(t)}}{\partial \theta}(r, 0) d r \sqrt{2 k(1-\cos \theta)} \int_{2}^{\infty} \sqrt{1-4 / \xi^{2}} \mathrm{e}^{\mathrm{i} k w(1-\cos \theta) \xi^{2} / 2} d \xi \\
& \left.-\frac{\sqrt{\pi}}{\sqrt{w}} \operatorname{erfc}\left[\mathrm{e}^{-\pi \mathrm{i} / 4} \sqrt{2 k w(1-\cos \theta)}\right] \int_{0}^{2}\left(\frac{a}{\tau}+\frac{\beta_{\infty}}{t^{\prime} \tau^{3 / 2}} \int_{0}^{\tau} \frac{\partial q^{(t)}}{\partial \theta}(r, 0) d r\right) \frac{d \tau}{\sqrt{2-\tau}}\right\} \\
& +\mathrm{e}^{-\mathrm{i} k\left(w-\delta M_{\infty}^{2}\right) \Gamma / 2+\mathrm{i} k w \epsilon \sigma_{l 1}(2,2 \pi)}\left\{\mathrm{e}^{\pi \mathrm{i} / 4} \frac{\sqrt{\pi}}{\sqrt{2 w}} \int_{0}^{2}\left(\frac{a}{\tau}+\frac{\beta_{\infty}}{t^{\prime} \tau^{3 / 2}} \int_{0}^{\tau} \frac{\partial q^{(t)}}{\partial \theta}(r, 2 \pi) d r\right)\right.
\end{aligned}
$$




$$
\begin{aligned}
& \times \mathrm{e}^{\mathrm{i} k w(1-\cos \theta) \tau} \operatorname{erfc}\left[\mathrm{e}^{-\pi \mathrm{i} / 4} \sqrt{2-\tau} \sqrt{k w(1-\cos \theta)}\right] d \tau \\
& +2 a \sqrt{k(1-\cos \theta)} \int_{2}^{\infty} \mathrm{e}^{\mathrm{i} k w(1-\cos \theta) \xi^{2} / 2}\left[\log [2]-\log \left[\xi^{2} / 2\right]-\log \left[1-\sqrt{1-4 / \xi^{2}}\right]\right] d \xi \\
& +\frac{\beta_{\infty}}{t^{\prime}} \int_{0}^{2} \frac{\partial q^{(t)}}{\partial \theta}(r, 2 \pi) d r \sqrt{2 k(1-\cos \theta)} \int_{2}^{\infty} \sqrt{1-4 / \xi^{2}} \mathrm{e}^{\mathrm{i} k w(1-\cos \theta) \xi^{2} / 2} d \xi \\
& \left.\left.\left.-\frac{\sqrt{\pi}}{\sqrt{w}} \operatorname{erfc}\left[\mathrm{e}^{-\pi \mathrm{i} / 4} \sqrt{2 k w(1-\cos \theta)}\right] \int_{0}^{2}\left(\frac{a}{\tau}+\frac{\beta_{\infty}}{t^{\prime} \tau^{3 / 2}} \int_{0}^{\tau} \frac{\partial q^{(t)}}{\partial \theta}(r, 2 \pi) d r\right) \frac{d \tau}{\sqrt{2-\tau}}\right\}\right]\right\}
\end{aligned}
$$

where

$$
P_{ \pm}=\frac{\mathrm{i}}{\sqrt{2}}(w-\delta) P_{l}(0 \pm) \mathrm{e}^{ \pm \mathrm{i} k\left(\left(w-\delta M_{\infty}^{2}\right) \Gamma / 2+w \epsilon V(0) \int_{0}^{2} q(r, 0 \pm) d r\right)+\mathrm{i} k\left(w-\delta M_{\infty}^{2}\right)\left(2+\alpha_{t}\right)+2 \mathrm{i} k \epsilon F_{R}(-\infty)} .
$$

We write the trailing-edge solution as

$$
h_{t}^{u} \sim \frac{D_{t}\left(\theta_{t}\right)}{k^{2} \sqrt{r_{t}}} \mathrm{e}^{\mathrm{i} k w \sigma_{t}\left(r_{t}, \theta_{t}\right)}
$$

The total far-field solution is given by

$$
h=h_{l}^{u}+h_{t}^{u}
$$

where $h_{l}^{u}$ is defined in (2.4.0.7). Once again, the total far-field solution can be written as a sum of the leading- and trailing-edge solutions

$$
h=\frac{\mathrm{e}^{\mathrm{i} k w \sigma_{l}}}{k^{3 / 2} \sqrt{r}}\left[D_{l}(\theta)-D_{t}(\theta) \frac{\mathrm{e}^{\mathrm{i} k w \sigma_{s}}}{\sqrt{k}}\right],
$$

where $\sigma_{s}$ is the phase shift between the leading- and trailing-edge ray fields, as measured by an observer in the far field at angle $\theta$ from the leading edge, and $D_{l, t}(\theta)$ are the far-field potential directivities for the leading and trailing edges. The phase shift, $\sigma_{s}$, is given by

$\sigma_{s}^{ \pm}=\sigma_{t}-\sigma_{l}=\frac{V(\theta)}{\beta_{\infty}}\left(2 \alpha_{i} \sin \theta+\cos \theta\left( \pm \alpha_{g} \pi+\beta_{\infty} \alpha_{t}\right)\right)+\left(2 \pm \frac{\alpha_{g} \pi}{\beta_{\infty}}+\alpha_{t}\right) \cos \theta-2 \epsilon F_{R}(-\infty)$,

where $\alpha_{g}$ is as defined in (1.4.1.3). The \pm denotes the phase shift above and below the aerofoil respectively, and is present due to the non-zero mean circulation. This phase shift, (2.7.0.6), varies from the sound-aerofoil phase shift, (1.5.0.2), only by the final different constant term, $-2 F_{R}(-\infty)$, now representing the total distortion of the gust as 


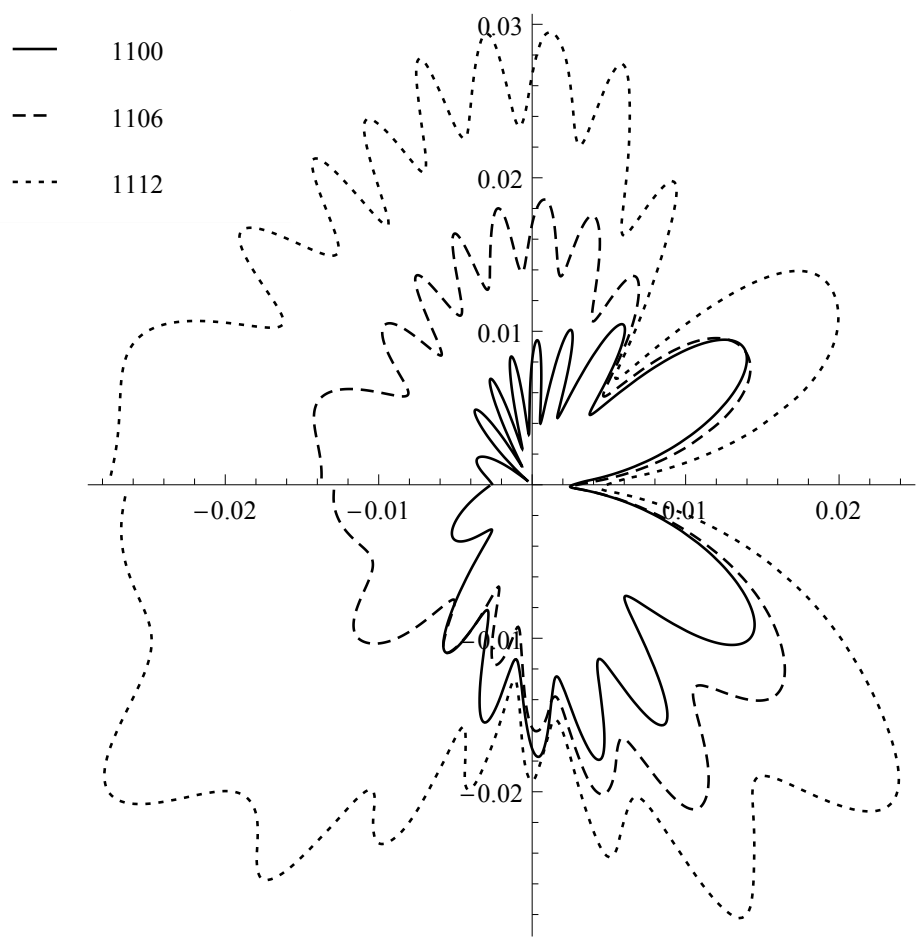

Figure 2.2: Far-field scattered pressure directivity for a NACA 4-digit aerofoil with $1 \%$ maximum camber at $10 \%$ chord length, $k=8, \alpha_{i}=0^{\circ}, M_{\infty}=0.7, \theta_{g}=45^{\circ}, k_{3}=0$. The thickness ratio is varied from $0 \%$ (flat plate) to $12 \%$.

it convects from far upstream to the leading edge of the aerofoil. Since these different terms are both constants, the interaction of the leading- and trailing-edge ray fields is very similar to the sound-aerofoil case caused by phase variations. Particularly, the increase or decrease of modulation of the far-field pressure magnitude is governed by similar physical processes.

\subsection{Results}

Here we present a selection of results for the unsteady pressure generated by the interaction of a gust with an aerofoil, as determined from the analytic solutions in this chapter. All figures are plotted in physical space unless otherwise specified, and the relationship between physical coordinates, $\left(r_{p}, \theta_{p}\right)$, and potential-streamline coordinates, $(r, \theta)$, is given by (1.6.1.1). We do not present as many far-field pressure directivity results here compared to the previous chapter because the physical processes governing the interaction of the leading- and trailing-edge fields is similar to the sound-aerofoil 
interaction case. These processes have been discussed in detail in Section 1.6. For all the plots presented in this section we set the spanwise gust velocity component, $A_{3}$, to zero, and $k_{t}=1$. We must mention that for gust-aerofoil interaction there is no Rayleigh distance as there are no Fresnel regions (the Rayleigh distance in sound-aerofoil interaction is the distance at which the Fresnel regions have overlapped and hence we see true far-field behaviour).

We also mention that our solution is only valid for $M_{\infty} \gtrsim 0.4$ since our asymptotic series encounters two problems for small values of $M_{\infty}$; first, individual terms in the asymptotic series which are multiplicatively dependent on the Mach number could become re-ordered leading to a potentially invalid asymptotic series. Second, the parameter $w$ is $O\left(M_{\infty}\right)$ for small Mach numbers. If the Mach number is too small, we can no longer treat $w k$ (which appears in the Helmholtz operator of the governing equation) as $O(k)$ which we have done throughout the analysis, leading to further potential failures of the presented asymptotic solution. To correct these problems one would need to construct a new asymptotic variable, dependent on $k, \epsilon$ and $M_{\infty}$, then solve for a new asymptotic series. For the same reason, a similar bound is required for the sound-aerofoil interaction problem in Chapter 1.

\subsubsection{Far-Field Acoustic Pressure}

All results in this section show the far-field acoustic pressure magnitude (also called the directivity) as determined by the terms in the square brackets of (2.7.0.5). We begin by comparing our solutions to those found by Tsai (1992, Figure 4.28) and Myers \& Kerschen (1997, Figure 4c). In Figure 2.3 we recreated Tsai's zero-camber result, and illustrate the effects of non-zero camber. In Figure 2.4 we recreate Myers and Kerschen's result and illustrate the effects of introducing thickness. It is clear from both Figures 2.3 and 2.4 that our new generalised solution limits to previously obtained asymptotic solutions for zero-camber and zero-thickness respectively.

The effects on the modulation of far-field scattered pressure directivity plots, due to varying parameters that are present in the phase shift, (2.7.0.6), are very similar to the effects seen in the previous chapter's results section, Section 1.6, since the phase shift for gust-aerofoil interaction is identical to that for sound-aerofoil interaction except for the final constant term, now $2 \epsilon F_{R}(-\infty)$. Clearly this term changes if we alter the aerofoil geometry, but since it is a constant with respect to $r$ and $\theta$, this variation is not explicitly seen in a given pressure directivity plot. The magnitude of the scattered pressure directivity depends on the directivity functions, $D_{l, t}(\theta)$, which see thickness, and 


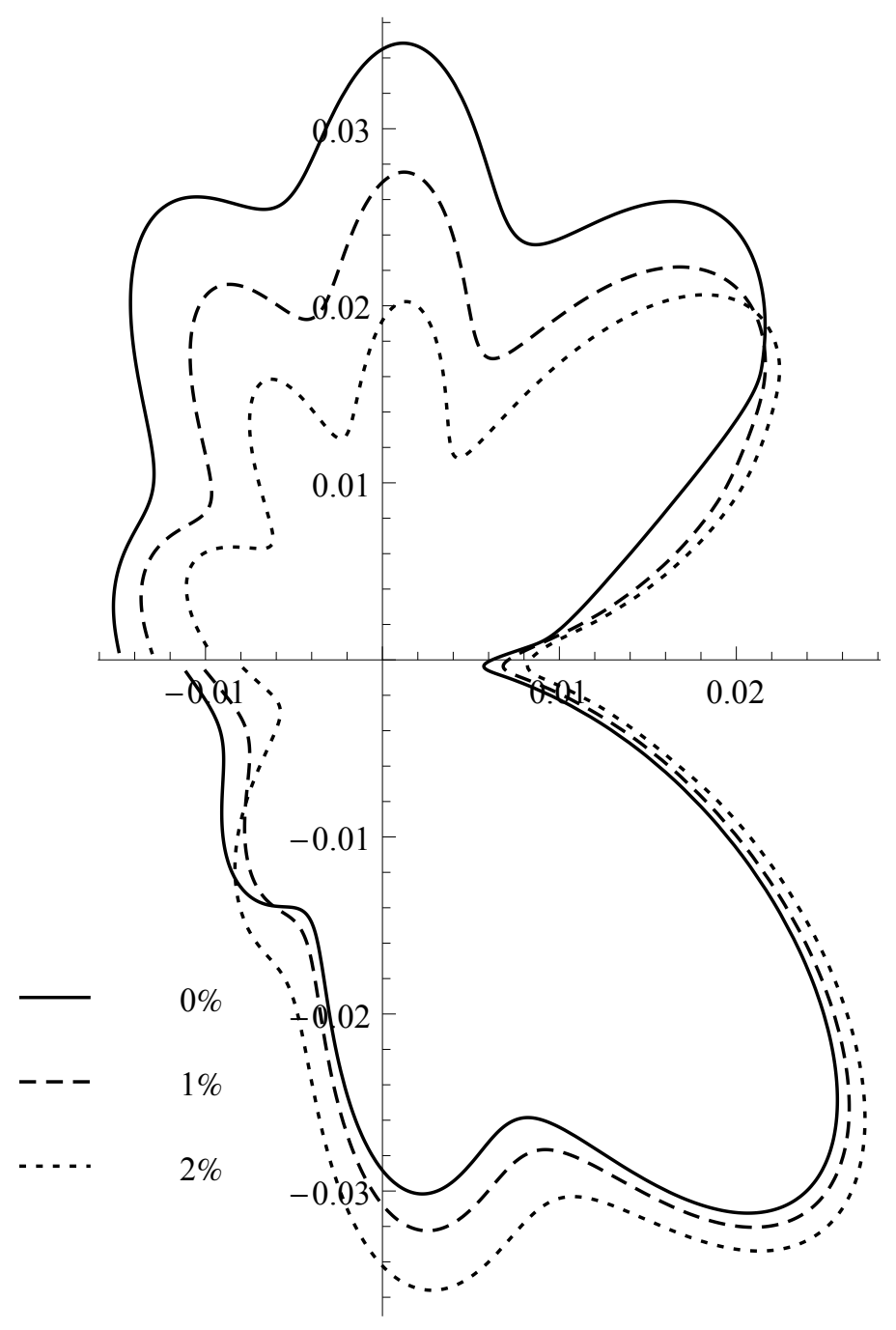

Figure 2.3: Far-field scattered pressure directivity for a $10 \%$ thick Joukowski aerofoil with $k=5, \alpha_{i}=0^{\circ}, M_{\infty}=0.6, k_{3}=0$, and $\theta_{g}=45^{\circ}$. Maximum camber is varied from $0 \%$ to $2 \%$, and the location of maximum camber is at $10 \%$ chord length. 


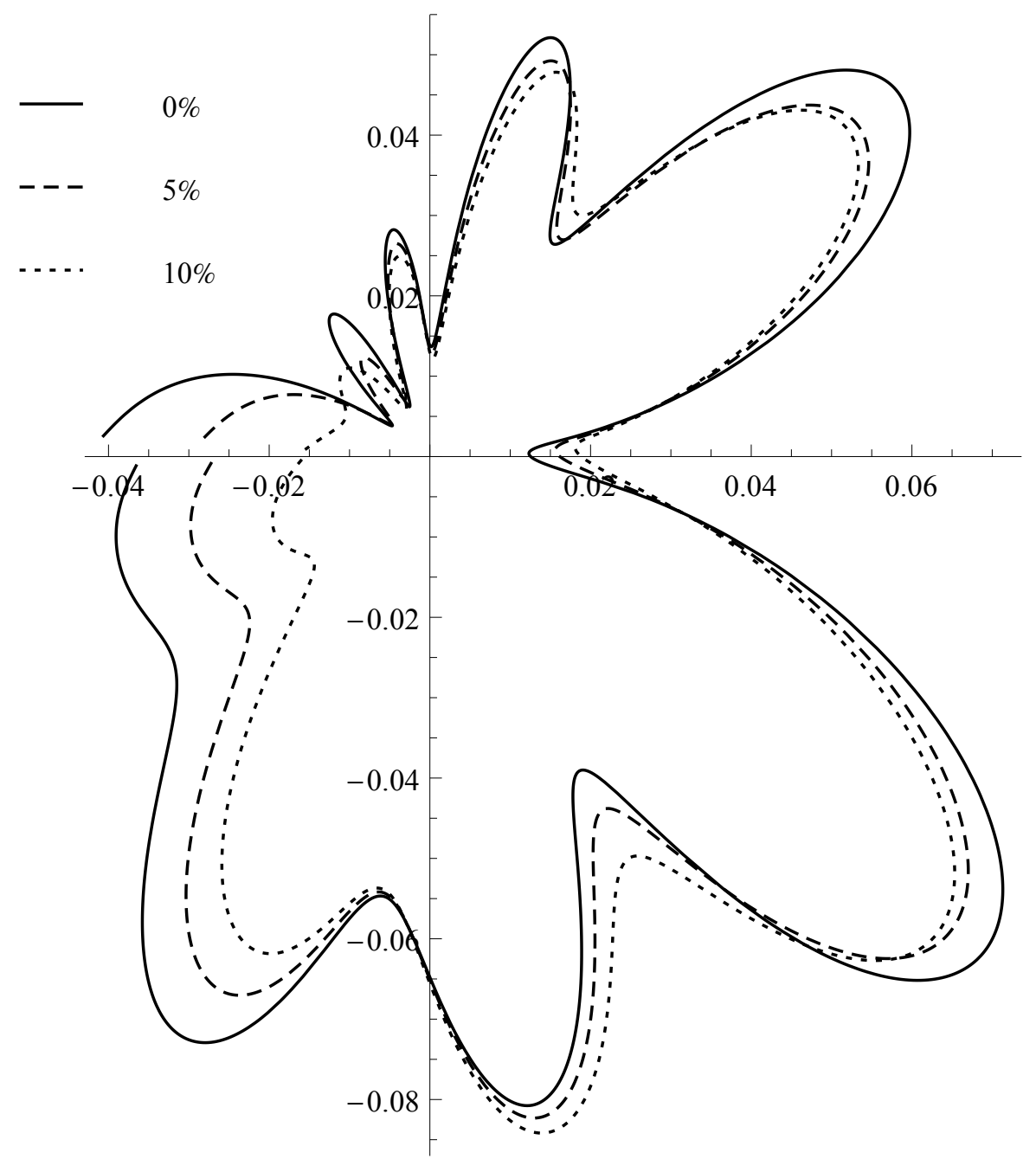

Figure 2.4: Far-field scattered pressure directivity for a 6-33 cambered aerofoil (as defined in Myers \& Kerschen (1997)) with $k=8, \alpha_{i}=0^{\circ}, M_{\infty}=0.5, k_{3}=0$, and $\theta_{g}=60^{\circ}$. Thickness is varied from $0 \%$ to $10 \%$. 
camber and angle of attack as essentially separate terms. The effects of varying thickness or camber and angle of attack have individually been considered by Tsai (1992) and Myers \& Kerschen $(1995,1997)$ respectively. Physical interpretations of altering all variables in our analysis for gust-aerofoil interaction are available in Tsai (1992) and Myers \& Kerschen (1997), as well as in Section 1.6 where we discussed the effects of varying the phase shift, $\sigma_{s}$. We therefore only mention specifically here results which have significant different interest to those from Chapter 1 or are not discussed in detail in Tsai (1992) or Myers \& Kerschen (1997).

Figure 2.2 illustrates the effects of altering the thickness of a NACA 4-digit aerofoil on the far-field acoustics. It is clear here (and can also be seen in some far-field soundaerofoil results) that the upstream pressure directivity has a small discontinuity across $\theta=\pi$. This is a result of the back-scatter from the trailing-edge ray field interacting with the leading-edge field. Whilst physically this discontinuity does not occur, asymptotically it does and it is $O\left(k^{-1}\right)$, hence is neglected during our analysis. To correct it, one would have to consider a further correction (i.e. at next order, $O(\epsilon)$ ) to the solution presented, where one imposes continuity of pressure across the upstream direction. The method would follow a similar approach to the trailing-edge solution, however would then result in a very small discontinuity across the trailing-edge!

Figure 2.5 shows the effects of altering the upstream gust angle, $\theta_{g}=\arctan \left(\beta_{\infty} k_{n} / k_{t}\right)$. There is no effect on the modulation of the directivity field because the gust angle is not present in the phase shift between the leading- and trailing-edge ray fields, however we see alterations in the magnitude of the pressure as the angle varies. This is due to the horizontal and vertical blocking of the gust velocity components, since the amplitude of the gust is dependent on the gust angle, as illustrated in (2.1.0.3). Downstream of the aerofoil there is a clear decrease in the pressure amplitude as the gust angle is increased, whereas upstream there is no general trend. The downstream effects are obvious because they relate to the leading-order flat-plate scattering; as the gust angle increases, the normal component of far-upstream magnitude of the gust velocity, $A_{n}$, decreases, therefore the blocking of the vertical gust momentum, seen in the flat-plate solution, $H_{0}$, reduces. The flat-plate directivity of $\cos \theta / 2$ is $O\left(A_{n}\right)$ along $\theta=0$, so the reducing $A_{n}$ is the main feature seen in the reducing pressure magnitude downstream, whilst the lower order effects of thickness and camber are not dominant. Upstream, however, the flat-plate directivity is small since $\cos \theta / 2 \rightarrow 0$ as $\theta \rightarrow \pi$, hence the effects of thickness and camber are effectively promoted and the directivity depends on complicated combinations of the aerofoil shape as well as the gust angle. Therefore directly upstream of the aerofoil, horizontal momentum blocking and non-linear flow effects at the leading edge 


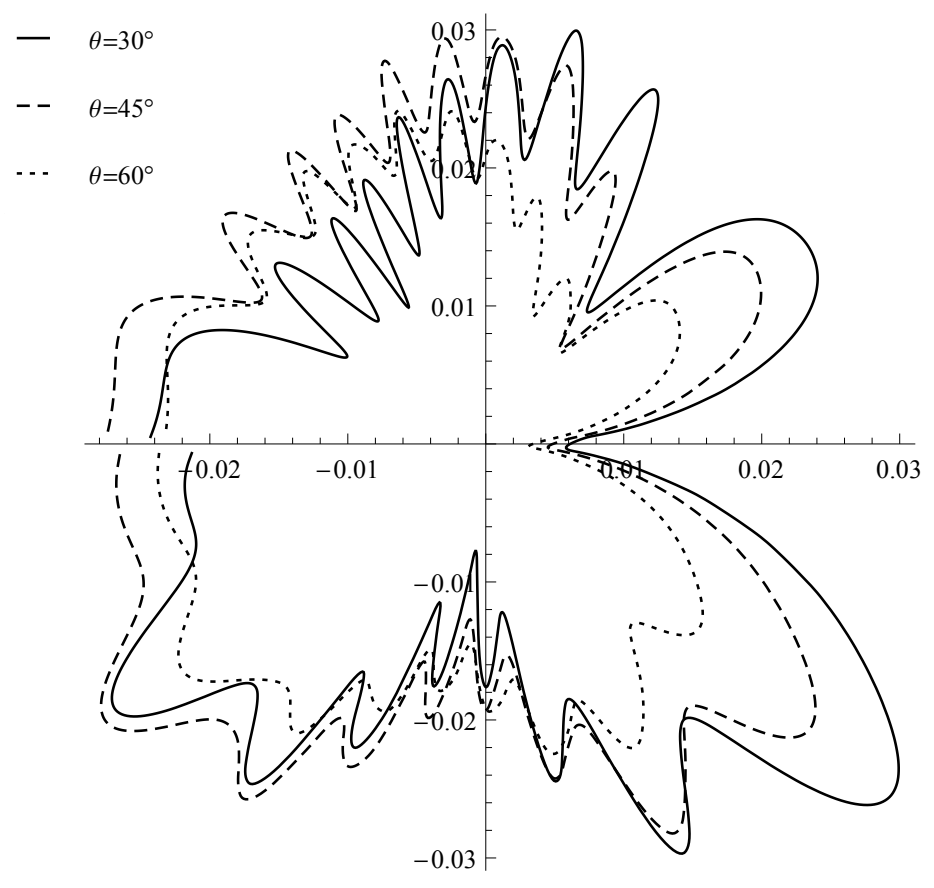

Figure 2.5: Far-field scattered pressure directivity for a NACA 1112 aerofoil with $k=8$, $\alpha_{i}=0^{\circ}, M_{\infty}=0.7, k_{3}=0$. The gust angle is varied from $30^{\circ}$ to $60^{\circ}$.

are more responsive to variations in the upstream gust angle than vertical momentum blocking.

Figure 2.6 shows the effects of varying the spanwise wavenumber, $k_{3}$. This has the result of altering $w$, hence we can only choose values of $k_{3}$ so that $w=O(1)$, ensuring our asymptotic series remains valid. As $w$ decreases, the frequency of both the leadingand trailing-edge fields decreases and so too does the phase shift between them (since all are proportional to $k w$ ). We therefore expect, and indeed observe, that the effect of varying $k_{3}$ is to alter the modulation of the far-field pressure directivity. Since $w$ remains $O(1)$ for all choices relating to this figure, we see little variation in the magnitude of the far-field pressure.

\subsubsection{Unsteady Surface Pressure}

By considering the leading- and trailing-edge inner solutions along with the leading-edge transition solution, we can obtain an approximation for the unsteady surface pressure, i.e. the pressure generated on the surface of the aerofoil due to the gust. The lift experienced by an aerofoil relates to the integral of the pressure difference across the blade, hence the ability to calculate the unsteady surface pressure imposed on a blade due to an incident 


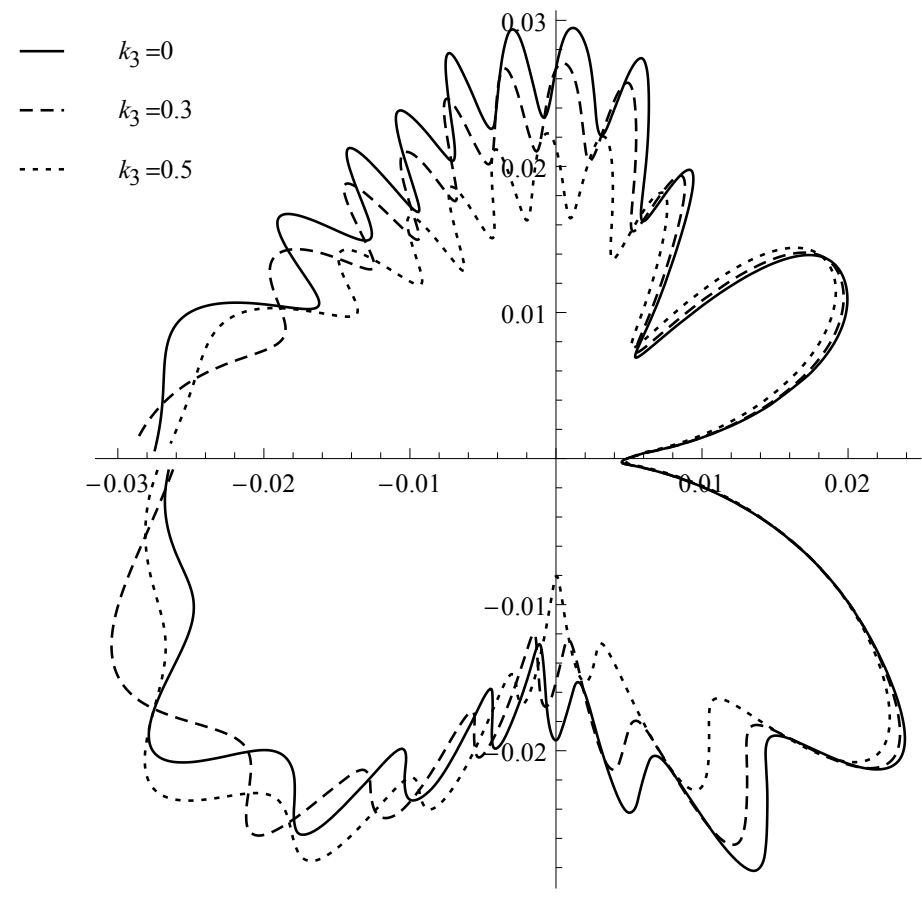

Figure 2.6: Far-field scattered pressure directivity for a NACA 1112 aerofoil with $k=8$, $\alpha_{i}=0^{\circ}, M_{\infty}=0.7, \theta_{g}=45^{\circ} . k_{3}$ is varied from 0 to 0.5 .

gust perturbation is very useful.

Figure 2.7a shows the effect of altering the angle of attack on the unsteady pressure jump across the aerofoil. As we decrease the angle of attack, the pressure jump increases across the entire length of the aerofoil. This does not contradict the well-known result that the steady lift on an aerofoil increases as we increase its angle of attack (up to the stall angle, but given we assume $\alpha_{i}=O(\epsilon)$ we are not concerned with stall angles), because we must remember that the plot shows only the unsteady pressure experienced by the aerofoil due to the gust, not the total pressure. We therefore see that an incident gust affects the lift on aerofoils at lower (or negative) angles of attack more than aerofoils at positive angles of attack.

Figure 2.7b illustrates the effect of changing the frequency of the incident gust. As seen in Figure 2.6, where we varied $k_{3}$ which is equivalent to a variation in frequency, increasing the frequency increases the modulation of the pressure. For the unsteady surface pressure jump this is still caused by the interaction between the leading- and trailing-edge fields. We observe a slight variation in magnitude of the pressure jump as we alter $k$, because the leading-edge field scales as $k^{-1}$ and the trailing-edge field scales as $k^{-3 / 2}$. 


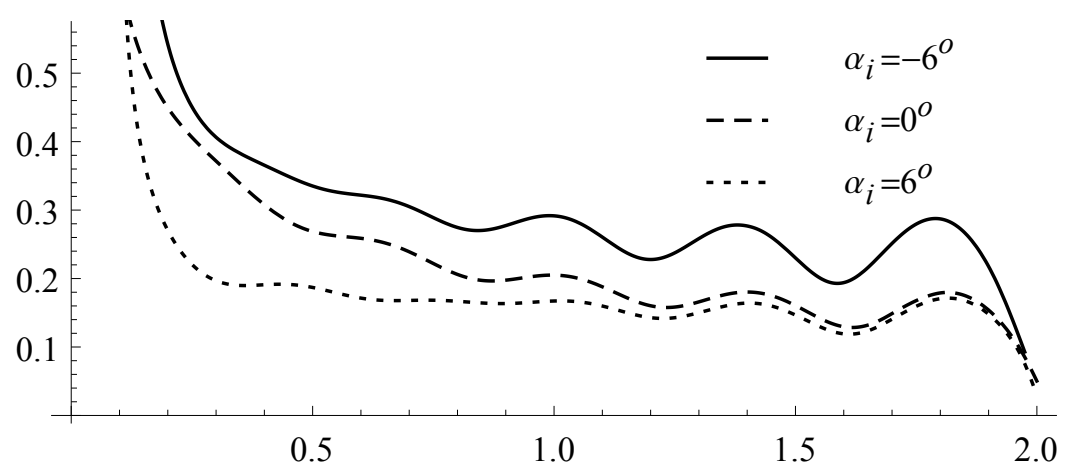

(a) NACA $1112, k=8$, varying $\alpha_{i}$.

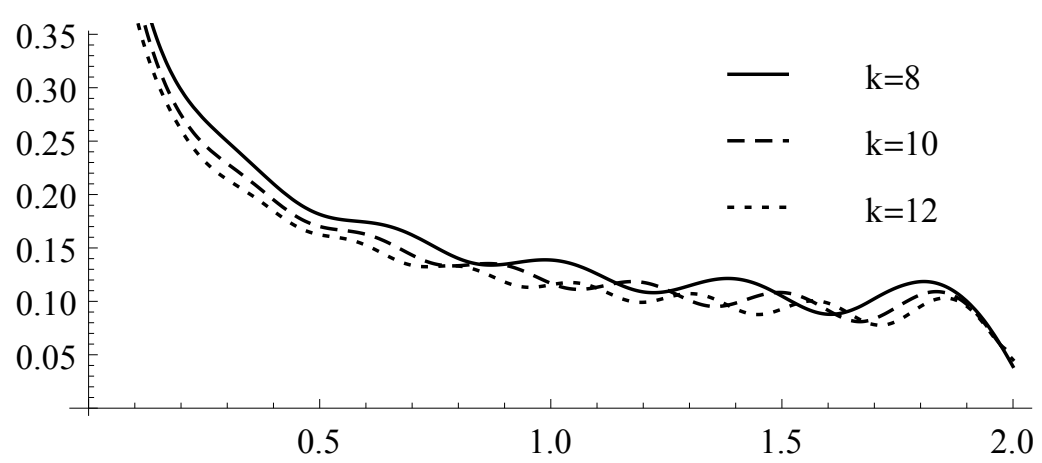

(b) NACA 1112, $\alpha_{i}=0^{\circ}$, varying $k$.

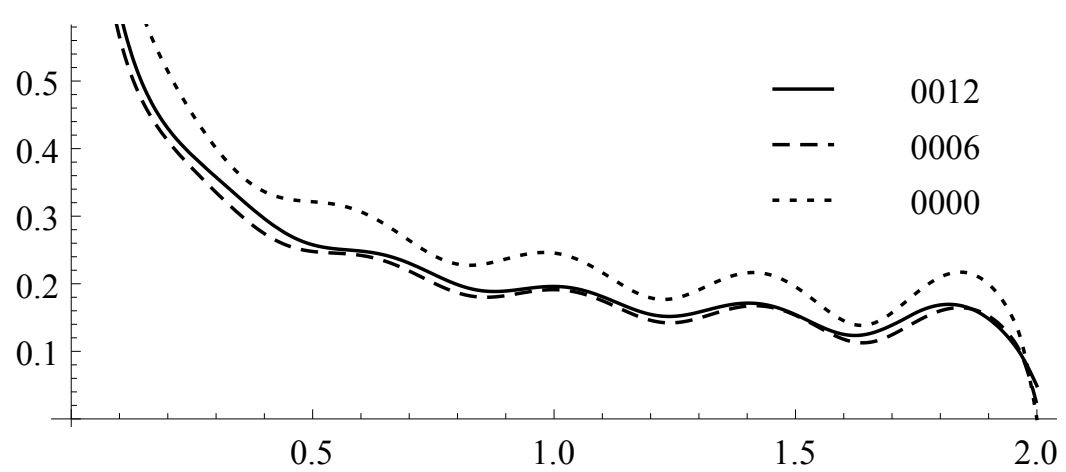

(c) $k=8, \alpha_{i}=0^{\circ}$, varying thickness.

Figure 2.7: Absolute value of the unsteady surface pressure jump across a NACA aerofoil, with $\theta_{g}=45^{\circ}, k_{3}=0$, and $M=0.6$. The horizontal axis denotes the chord position, and the vertical axis measures the non-dimensionalised pressure jump. 


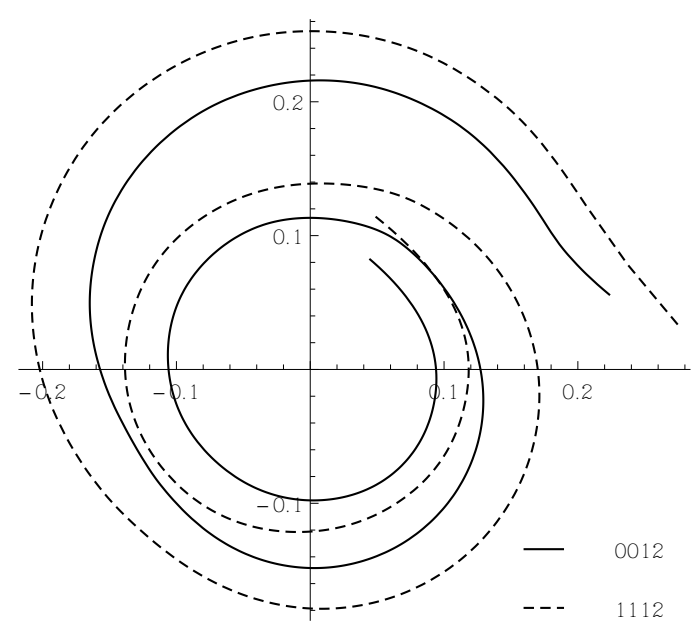

Figure 2.8: Unsteady lift on a NACA 1112 aerofoil, with $\theta_{g}=45^{\circ}$ and $\alpha_{i}=0^{\circ} . k$ varies from 8 at the outermost point on the spiral, to 20 at the innermost point on the spiral. Axes show the real and imaginary parts of the non-dimensionalised lift.

Figure 2.7c shows the effect of altering the thickness of the aerofoil; there is little difference between a $12 \%$ and a $6 \%$ thick aerofoil. However, there is a notable difference between aerofoils with non-zero thickness and flat plates; the flat plate generates a larger unsteady pressure jump than the thick aerofoils. For a thick aerofoil the gust is blocked horizontally by the bluff nose and the steady flow is slowed by the bluff aerofoil. A flat plate can only block the gust vertically and no flow slowing occurs. We see from Figure 2.4 that increasing thickness predominantly decreases the far-field pressure magnitude upstream of the aerofoil, but has little effect elsewhere, and away from the upstream region the difference between the $10 \%$ - and 5\%-thick aerofoils is smaller than the difference between the zero-thickness and 5\%-thick aerofoils.

As the frequency of the gust increases, we expect the unsteady lift on the aerofoil (i.e. the lift caused by the perturbation gust rather than the background steady flow) to decrease because the unsteady response by the aerofoil scales with inverse powers of $k$. Figure 2.8 illustrates this, but we also see that the complex value of the unsteady lift forms a spiral as we vary $k$. This is in agreement with the trend exhibited by the Sears function (Sears, 1941) which is proportional to the unsteady lift generated by a flat plate interacting with a sinusoidal gust in two-dimensional incompressible flow. We do not see lift values with large positive real parts for the smaller values of $k$ in Figure 2.8 (something that is observed with the Sears function) because our solution is restricted to the high-frequency regime therefore we do not expect to recover any of the low-frequency behaviour that is exhibited by the Sears function. 
We note here that there is an integrable singularity in the unsteady surface pressure at the leading edge predicted by the asymptotic solution as seen in Figure 2.7. This singularity violates the asymptotic assumption that the unsteady flow is much smaller than the steady flow, hence in a small region close to the nose the solution is not valid (but it does not invalidate any of our far-field solutions. It only invalidates our surface pressure solutions in a small region close to $x=0$ ). This singularity is caused by the stagnation point of the steady flow, therefore we should consider a region around the stagnation point more carefully. To determine the size of this region we consider the true placing of the stagnation point for a lifting aerofoil. It lies at an $O\left(\epsilon^{2}\right)$ distance from the leading edge, however due to the approximations enforced during the asymptotic work, we treat this point as if it were the same as the point directly at the leading edge. This results in a square root type singularity in the surface pressure, $x^{-1 / 2}$, which should actually be $\left(x+\epsilon^{2}\right)^{-1 / 2}$ which is non-singular (see Van Dyke (1975) for further details). This scaling implies that there should be a further asymptotic region close to the leading edge and stagnation point of the aerofoil that we need to treat differently in order to rectify the surface pressure singularity. If we were to introduce this new region to the solution in this chapter, we would have to retain terms of $O\left(\epsilon^{2}\right)$ and therefore have to create a solution with many more terms than we have done currently. This is clearly not the optimal solution to the problem, therefore later, in Chapter 4, we choose a slightly different approach to solve for the flow around the stagnation point. The solution we obtain, however, can still be thought of as an "inner-inner" region to the current problem because it is shown to be consistent with the solutions in this chapter.

\subsubsection{Comparison with Numerical Results}

As mentioned in the introduction to this dissertation and Section 1.6.2, current computational schemes tend to operate well for low- to mid-range gust frequencies only. Figure 2.9 illustrates the comparison of asymptotic results obtained here to a numerical solution obtained by Ray Hixon (from work relating to Hixon et al. (2006), personal communication). We see that the agreement between the numerics and the asymptotics increases with distance from the aerofoil, and the greatest discrepancy between the two results always lies in the second quadrant, upstream and above the aerofoil. The results agree less as we get closer to the aerofoil, because during the analysis of the leading-edge transition solution (Section 2.4) we take an asymptotic expansion of this solution with respect to $\phi$, and neglect any powers of $O\left(\phi^{-1}\right)$. This approximation is used when finding the trailing-edge outer solution since we are primarily concerned with the far-field 
acoustics (recall, the trailing-edge solutions rescatter the leading-edge transition solution, hence despite the leading-edge transition solution itself being negligible at a distance of $O\left(k^{-1 / 2}\right) \approx 0.45$ away from the aerofoil, the rescattering of that solution can have an effect everywhere). At distances of just two chord lengths away from the aerofoil, the assumption that we can neglect $O\left(\phi^{-1}\right)$ is invalid. As we go further from the aerofoil, to five chord lengths away, this assumption almost coincides with assuming $O(\epsilon)$ terms are negligible, hence the result is more reliable and we indeed see a closer match between the asymptotic and the numeric solutions.

We also compare the unsteady surface pressure that is obtained asymptotically with a numerical solution using a similar numerical scheme to the one presented in Hixon et al. (2006); see Figure 2.10. Here we use the expression for the leading-edge transition solution that is valid close to the aerofoil so we do not encounter the same validity problems we discussed in the previous paragraph (i.e. neglecting $O\left(\phi^{-1}\right)$ ). The comparison is for $k=3 / \beta_{\infty} \approx 3.5$ however, which is certainly not large enough to be considered "high-frequency" in our analysis. Nevertheless, the overall trends exhibited by both the numeric and asymptotic solutions, such as the crossover points and general curve shape, agree, hence this is a good indication that both results, when in a suitable frequency range, yield credible results.

There are several issues hindering our comparisons. First, the parameters at which the comparisons are made are considered in the high-frequency regime for the numerical scheme (at which computation becomes difficult and errors may occur), but are only just within the high-frequency regime specified by the analysis presented here. Secondly, our asymptotic solution is truncated at two orders of magnitude in the amplitude and the phase, hence any additional terms, which are at least $O(\epsilon)=O\left(k^{-1}\right)$ with respect to the leading-order term have been neglected. For the values of $k$ and $\epsilon$ chosen in these comparisons, the neglected terms of size $O\left(k^{-1}\right)$ are almost the same order of magnitude as the retained terms of size $O(\epsilon \sqrt{k})$.

A further difference between Hixon's numerical solution and our asymptotic solution is elementary; Hixon solves the fully non-linear Navier-Stokes equations for a given background flow with incident gust perturbation. Whilst the non-linear terms are indeed small for this code, near the nose of the aerofoil they have a non-negligible effect on the non-uniform flow. Since the flow around both the nose and trailing edge are fundamental to the asymptotic results, we anticipate that any difference between the flows considered numerically and asymptotically result in differences in the scattered acoustics.

To compare our results against a computational aeroacoustics method solving the same linearised Euler equations, we turn to Gill et al. (2013); in this paper the leading- 


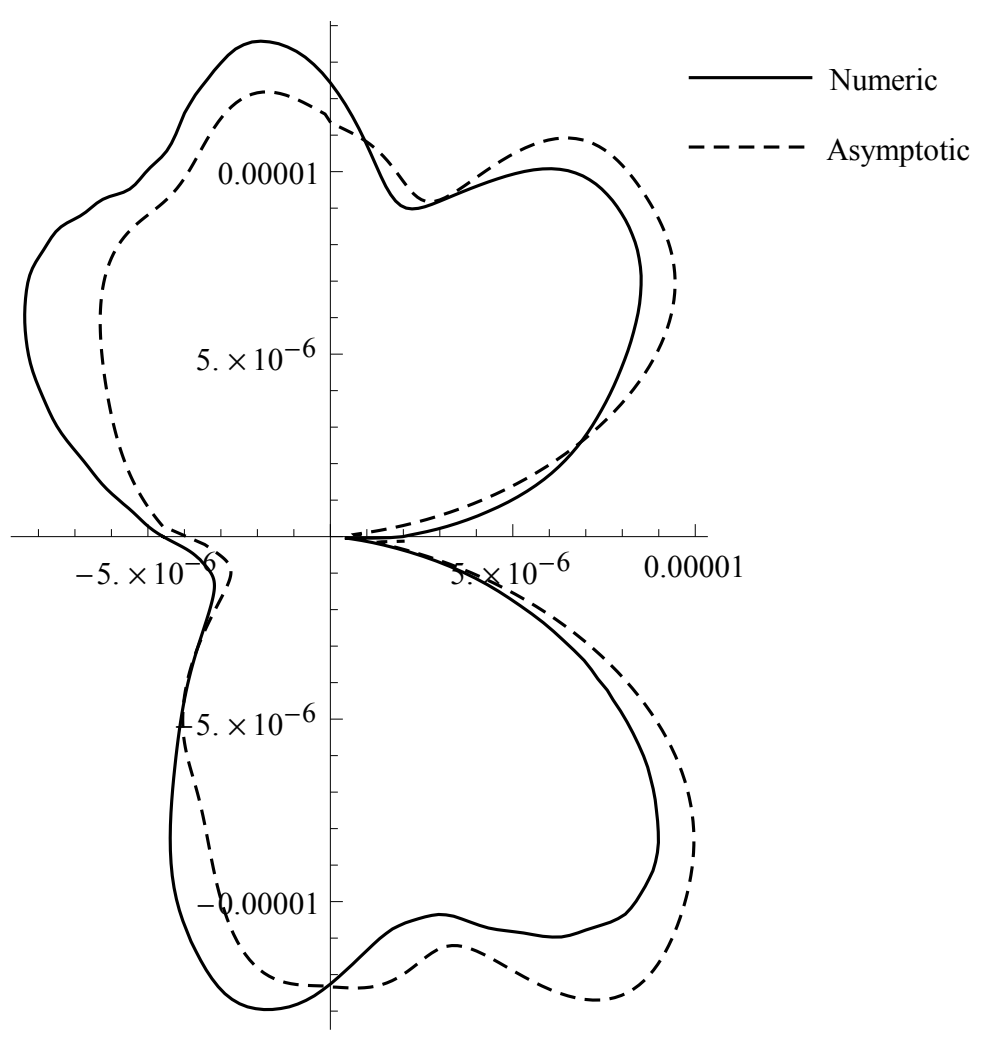

(a) Scattered pressure directivity at a distance of 3 chord lengths from the centre of the aerofoil.

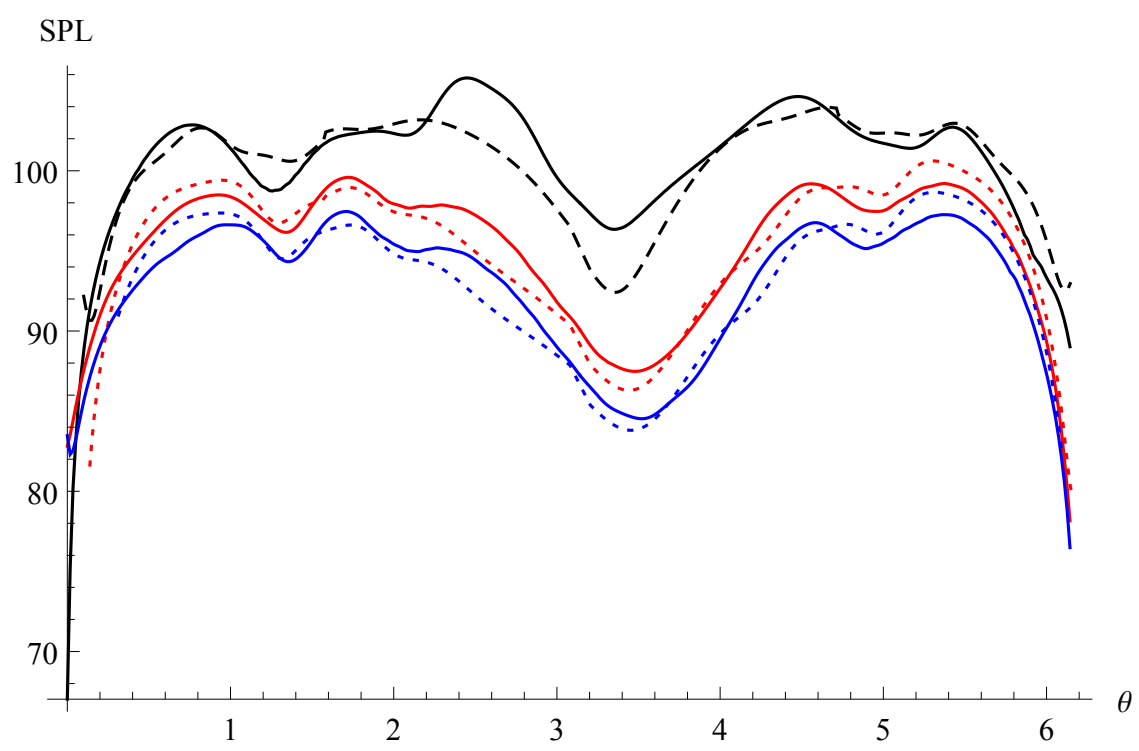

(b) Sound pressure levels in $\mathrm{dB}$ at various distances away from the centre of the aerofoil; black $=1$ chord, red $=3$ chords, blue $=5$ chords.

Figure 2.9: Comparison of asymptotic and numeric results for a NACA 0012 aerofoil at $M=0.5, k=5 / \beta_{\infty}, k_{t}=1, \alpha_{i}=0, k_{3}=0$, and $\theta_{g}=45^{\circ}$. Numeric results provided by Ray Hixon (pers. comm. with permission) are solid lines, asymptotic results are dashed lines. 

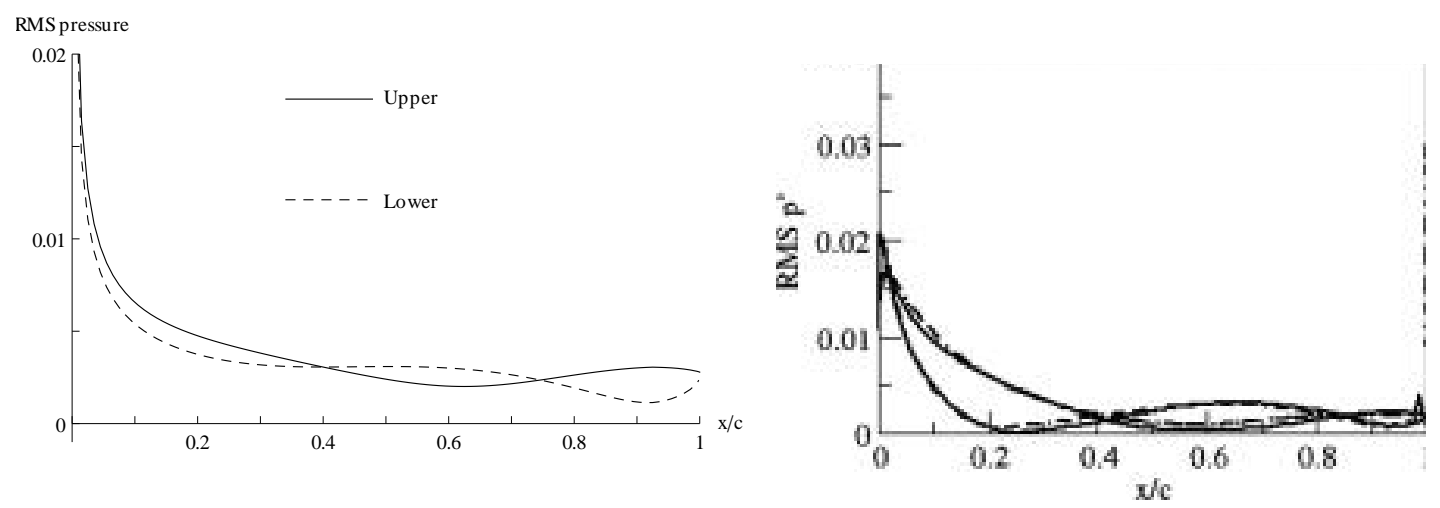

(a) Asymptotic Results.

(b) Numerical results from Hixon et al. (2006) (with permission).

Figure 2.10: Perturbation RMS surface pressure on a symmetric Joukowski aerofoil of $12 \%$ thickness, at $M=0.5, k=3 / \beta_{\infty}, k_{3}=0$, and $\theta_{g}=45^{\circ}$. The $x$-axis denotes the position along the aerofoil chord as a fraction of total chord length.

and trailing-edge noise sources are analysed close to the solid body, then radiated to the far field using a Ffowcs Williams-Hawkings solver (Ffowcs Williams \& Hawkings, 1969). Figure 2.12 shows the comparison between the computational solution at a radial distance of 25 and our asymptotic far-field solution. James Gill has also provided us with a flat-plate solver, allowing us to reproduce far-field directivities using Amiet's method (Amiet, 1975). In Figure 2.11 we see the very good agreement between our far-field asymptotic solution for the pressure directivity and Amiet's solution. In Figure 2.12 we see good agreement between Gill's numeric and asymptotic solutions in the trailingedge region (the first quadrant), but the agreement is less convincing in the leading-edge region. We are yet to identify why this discrepancy in the leading-edge region occurs.

\subsection{Conclusions}

In this chapter we have significantly extended and generalised the gust-aerofoil interaction problems presented by Myers \& Kerschen (1997) and Tsai (1992); it is now possible to find the far-field noise generated by a lifting aerofoil with small but non-zero thickness interacting with a gust in a background steady uniform flow. Turbulence is commonly decomposed into a Fourier series of gust components, and each Fourier frequency is considered individually; it is therefore important to be able to access acoustic solutions at high frequencies in order to accurately describe the noise generated by an aerofoil interacting with turbulence in a uniform steady flow. Since numerical schemes commonly struggle at high frequencies $(k \gtrsim 5)$, this work will allow acoustic solutions to be found 


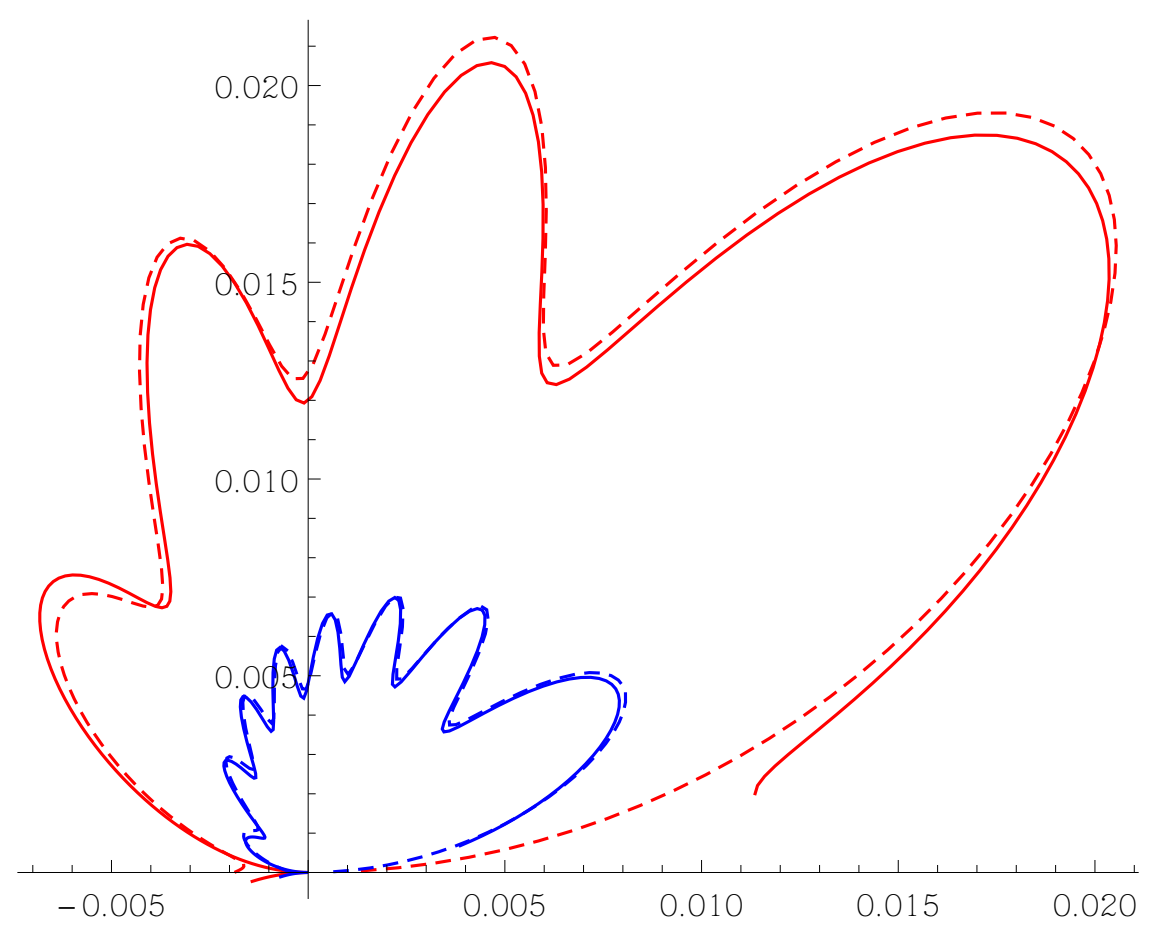

(a) $M=0.6$, red has $k=6.25$, blue has $k=12.75$.

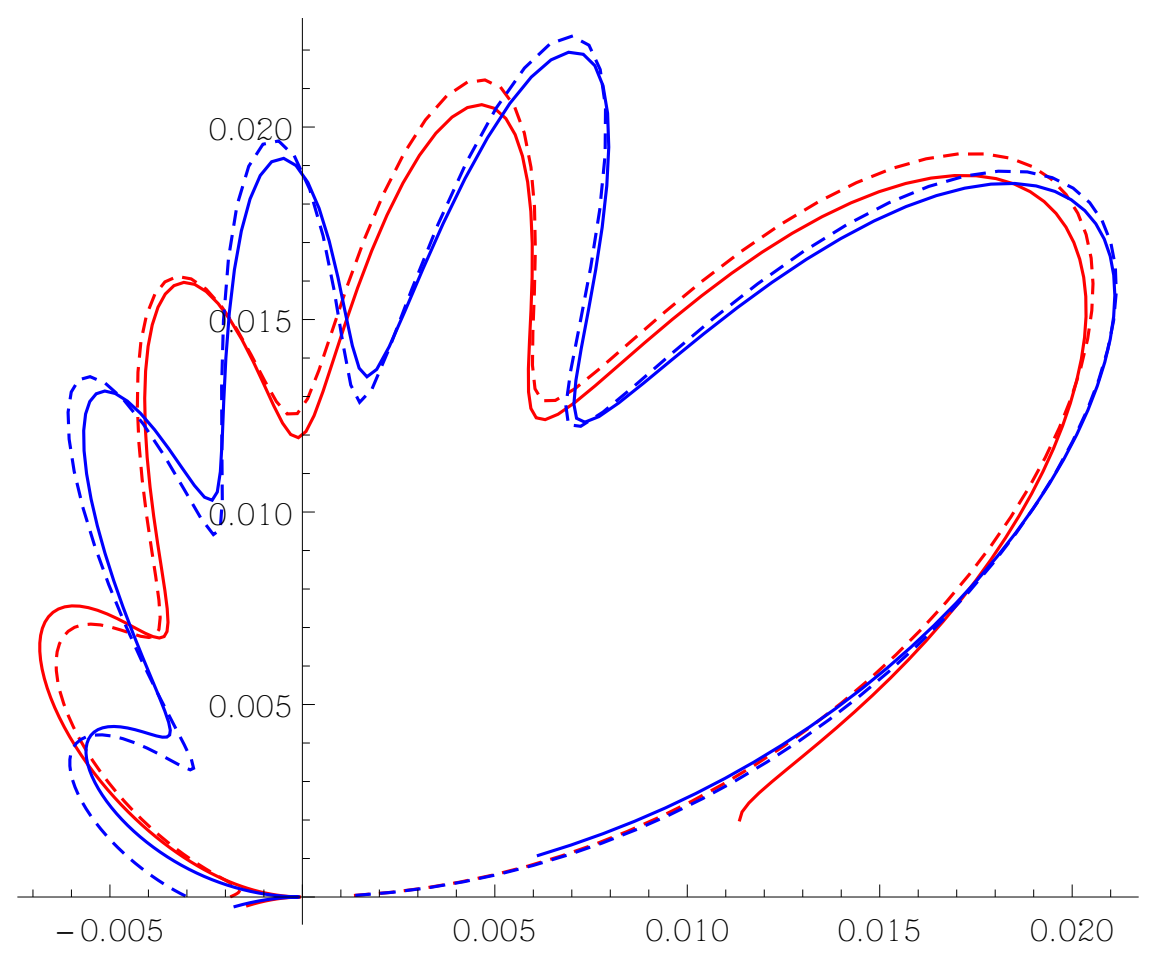

(b) $k=6.25$, red has $M=0.6$, blue has $M=0.65$.

Figure 2.11: Comparison of asymptotic and Amiet far-field pressure directivities for a flat plate with $\theta_{g}=45^{\circ}, k_{t}=1, k_{3}=0$, and $\alpha_{i}=0^{\circ}$. Numerical results are solid lines, whilst asymptotic results are dashed lines. Amiet solver provided by James Gill (pers. comm. with permission). 


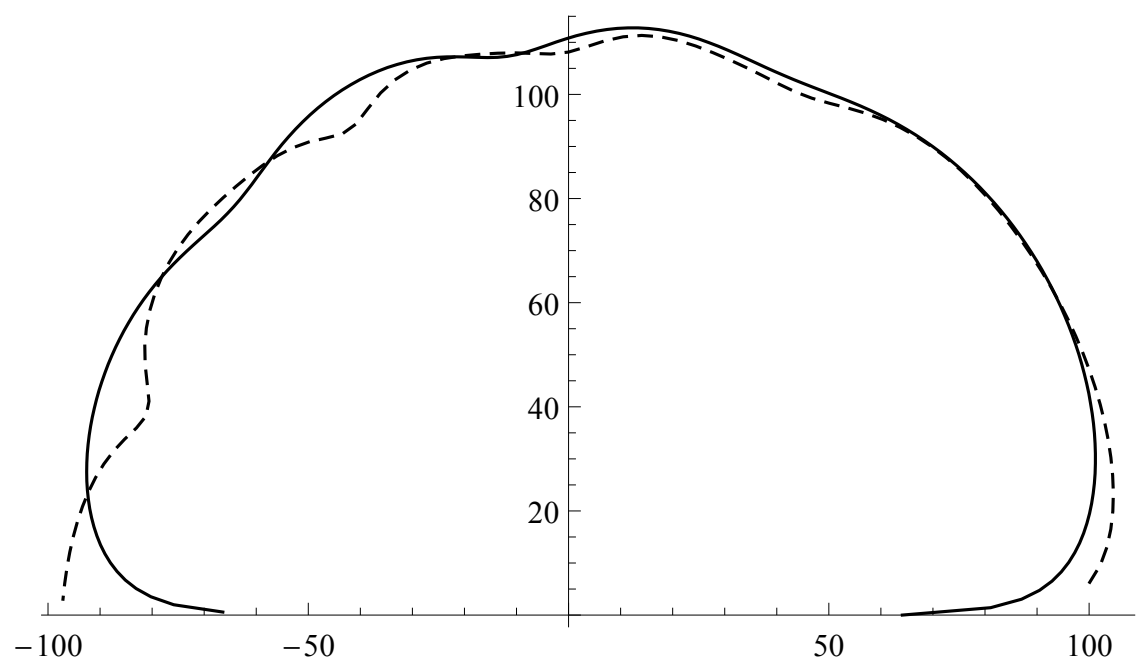

(a) $k=2 \pi$.

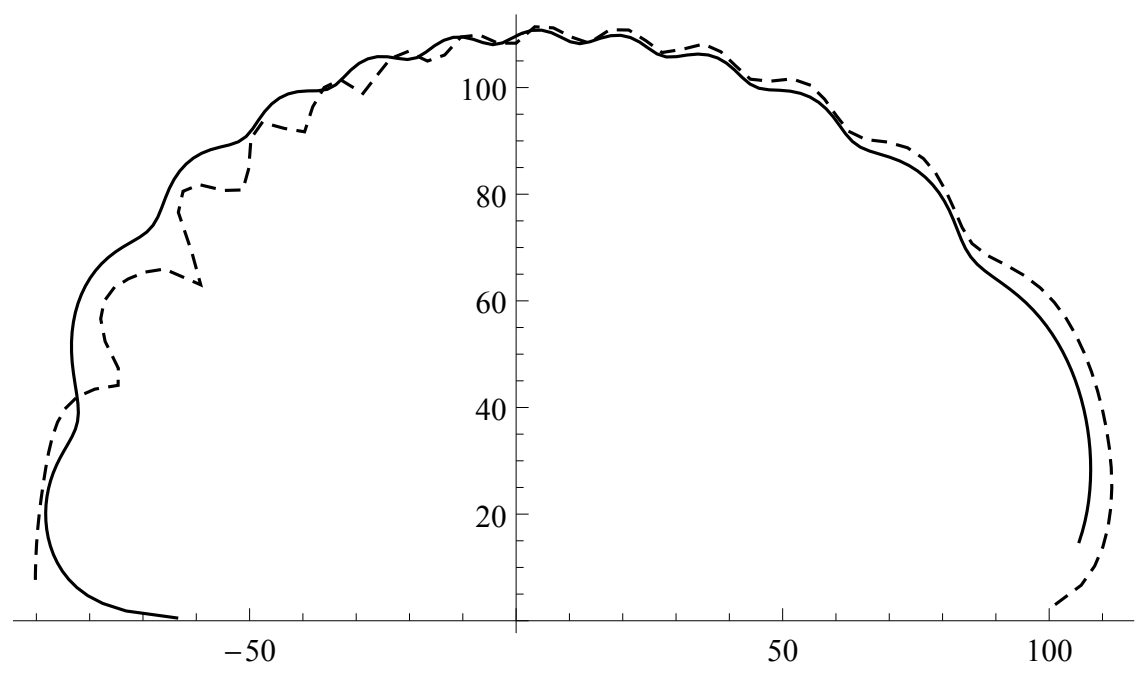

(b) $k=6 \pi$.

Figure 2.12: Comparison of asymptotic and numeric far-field SPL in dB for a NACA 0012 aerofoil with $\theta_{g}=45^{\circ}, k_{t}=1, k_{3}=0$, and $\alpha_{i}=0^{\circ}$. Numerical results are solid lines courtesy of James Gill (pers. comm. with permission), whilst asymptotic results are dashed lines. 
at a wider range of frequencies than are currently available; computational codes can implement the far-field approximations as boundary conditions rather than imposing non-reflective boundary conditions, which are increasingly difficult to resolve for a given aerofoil and background flow as the frequency of the initial gust increases.

One important point we have uncovered in this chapter can be seen in Figures 2.4 and $2.7 \mathrm{c}$; for high-frequency gusts, the introduction of thickness to a flat-plate aerofoil has a big impact on the unsteady surface pressure and the far-field scattered pressure (when compared with the difference in pressure generated by two aerofoils with non-zero thickness) due to the shift from purely horizontal momentum blocking, to a mixture of horizontal and vertical momentum blocking, as well as unsteady flow effects around a bluff nose. Figure 2.7c illustrates that modelling thin aerofoils by flat plates is not sufficiently accurate when considering high-frequency interactions therefore there is a clear need to have results available for gust-aerofoil interaction for real geometry aerofoils with non-zero thickness. For low-frequency gust-aerofoil interactions, the flat-plate model is accurate since the effects of thickness are very small, hence we have not been concerned with the low-frequency case.

We have also seen in Section 2.8.2 that the asymptotic results predict an unsteady pressure singularity near the nose of a thick aerofoil. This violates the small perturbation assumption initially posed at the start of the analysis, hence the results are not asymptotically valid on the surface of a thick aerofoil near the leading edge. It does not affect the far-field results at all. A more detailed asymptotic investigation is required close to the stagnation point of the steady uniform flow in order to accurately assess the behaviour there and hopefully eliminate any violation of initial assumptions. We do this later, in Chapter 4, by considering the leading-edge region in more detail. 


\section{List of New Symbols for Chapter 2}

$\boldsymbol{A}$ upstream velocity amplitude for the incident gust, $=\left(A_{t}, A_{n}, A_{3}\right)$ in $\left(\phi, \psi, x_{3}\right)$ space.

$A_{t}^{*} \quad A_{t}-B$

$F_{R}$ real part of $F(z)$.

$g \quad$ drift function in $(\phi, \psi)$ space.

$h_{c} \quad$ complementary solution in the outer region, $=f(k|\psi|, \phi) \mathrm{e}^{\mathrm{i} k \Omega(\phi, 0)}$.

$h_{p} \quad$ particular solution in the outer region.

$\boldsymbol{k} \quad$ wavevector for the incident gust perturbation, $=\left(k_{t}, k_{n}, k_{3}\right)$.

$s \quad$ entropy perturbation, $=2 B$ far upstream.

$S \quad$ amplitude of the source term of the governing equation generated by the incident gust.

$\delta \quad k_{t} / \beta_{\infty}^{2}$

$\Omega \quad$ phase of the source term of the governing equation generated by the incident gust.

$\theta_{g} \quad$ upstream gust angle far upstream, $=\arctan \left(\beta_{\infty} k_{n} / k_{t}\right)$. 


\section{Appendix A}

The analysis for the leading-edge inner term, $H_{3}$, is given here. It follows the procedure set out by Tsai (1992) however includes corrections to his stated results, hence most calculations are included. We choose a particular solution to (2.2.4.1) of the form

$$
\begin{array}{r}
H_{3 p}^{*}=\frac{A_{n} w^{2} a \operatorname{sgn}(\Psi) \sqrt{R}}{\pi \sqrt{\delta+w}}\left(\cos \theta / 2 \int_{-\infty}^{\infty} C_{1}(\lambda) \mathrm{e}^{a(\lambda, \Phi, \Psi)} d \lambda+\sin \theta / 2 \int_{-\infty}^{\infty} C_{2}(\lambda) \mathrm{e}^{a(\lambda, \Phi, \Psi)} d \lambda\right. \\
\left.+\frac{\cos \theta / 2}{R} \int_{-\infty}^{\infty} C_{3}(\lambda) \mathrm{e}^{a(\lambda, \Phi, \Psi)} d \lambda+\frac{\sin \theta / 2}{R} \int_{-\infty}^{\infty} C_{4}(\lambda) \mathrm{e}^{a(\lambda, \Phi, \Psi)} d \lambda\right) . \quad \text { (A.1a) }
\end{array}
$$

Applying the Helmholtz operator yields

$$
\begin{aligned}
D\left(H_{3 p}^{*}\right)= & \frac{A_{n} w^{2} a \operatorname{sgn}(\Psi)}{\pi \sqrt{\delta+w}}\left(\frac{\cos \theta / 2}{\sqrt{R}} \int_{-\infty}^{\infty}\left(-\mathrm{i} \lambda C_{1}(\lambda)-\operatorname{sgn}(\Psi) \sqrt{\lambda^{2}-w^{2}} C_{2}(\lambda)\right) \mathrm{e}^{a(\lambda, \Phi, \Psi)} d \lambda\right. \\
& +\frac{\sin \theta / 2}{\sqrt{R}} \int_{-\infty}^{\infty}\left(-\operatorname{sgn}(\Psi) \sqrt{\lambda^{2}-w^{2}} C_{1}(\lambda)+\mathrm{i} \lambda C_{2}(\lambda)\right) \mathrm{e}^{a(\lambda, \Phi, \Psi)} d \lambda \\
& +\frac{\cos 3 \theta / 2}{R^{3 / 2}} \int_{-\infty}^{\infty}\left(\mathrm{i} \lambda C_{3}(\lambda)-\operatorname{sgn}(\Psi) \sqrt{\lambda^{2}-w^{2}} C_{4}(\lambda)\right) \mathrm{e}^{a(\lambda, \Phi, \Psi)} d \lambda \\
& \left.+\frac{\sin 3 \theta / 2}{R^{3 / 2}} \int_{-\infty}^{\infty}\left(\operatorname{sgn}(\Psi) \sqrt{\lambda^{2}-w^{2}} C_{3}(\lambda)+\mathrm{i} \lambda C_{4}(\lambda)\right) \mathrm{e}^{a(\lambda, \Phi, \Psi)} d \lambda\right) .
\end{aligned}
$$

Solving for $C_{1,2,3,4}(\lambda)$ gives

$$
\begin{aligned}
H_{3 p}^{*}= & \frac{A_{n} a}{\pi \sqrt{\delta+w}}\left(\sqrt{R} \cos \theta / 2 \int_{-\infty}^{\infty} \sqrt{\lambda-w}(\lambda+\delta)\left[\frac{1}{(\lambda+\delta)^{2}}-\frac{(\gamma+1) M_{\infty}^{4}}{2 \beta_{\infty}^{4} w^{2}}\right] \mathrm{e}^{a(\lambda, \Phi, \Psi)} d \lambda\right. \\
& -\mathrm{i} \operatorname{sgn}(\Psi) \sqrt{R} \sin \theta / 2 \int_{-\infty}^{\infty} \frac{\lambda(\lambda+\delta)}{\sqrt{\lambda+w}}\left[\frac{1}{(\lambda+\delta)^{2}}-\frac{(\gamma+1) M_{\infty}^{4}}{2 \beta_{\infty}^{4} w^{2}}\right] \mathrm{e}^{a(\lambda, \Phi, \Psi)} d \lambda \\
& -\frac{(\gamma+1) M_{\infty}^{4}}{4 \beta_{\infty}^{4} w^{2}} \frac{\mathrm{i} \cos \theta / 2}{\sqrt{R}} \int_{-\infty}^{\infty} \sqrt{\lambda-w} \mathrm{e}^{a(\lambda, \Phi, \Psi)} d \lambda \\
& \left.+\operatorname{sgn}(\Psi) \frac{(\gamma+1) M_{\infty}^{4}}{4 \beta_{\infty}^{4} w^{2}} \frac{\sin \theta / 2}{\sqrt{R}} \int_{-\infty}^{\infty} \frac{\lambda}{\sqrt{\lambda+w}} \mathrm{e}^{a(\lambda, \Phi, \Psi)} d \lambda\right) .
\end{aligned}
$$

To evaluate this behaviour at small $R$ we must consider both local and global expansions. Define

$$
\begin{aligned}
& I_{2}(R, \theta)=\int_{-\infty}^{\infty} \sqrt{\lambda-w}(\lambda+\delta)\left(\frac{1}{(\lambda+\delta)^{2}}-\frac{(\gamma+1) M_{\infty}^{4}}{2 \beta_{\infty}^{4} w^{2}}\right) \mathrm{e}^{a(\lambda, R, \theta)} d \lambda, \\
& I_{3}(R, \theta)=\int_{-\infty}^{\infty} \frac{\lambda(\lambda+\delta)}{\sqrt{\lambda+w}}\left(\frac{1}{(\lambda+\delta)^{2}}-\frac{(\gamma+1) M_{\infty}^{4}}{2 \beta_{\infty}^{4} w^{2}}\right) \mathrm{e}^{a(\lambda, R, \theta)} d \lambda, \\
& I_{4}(R, \theta)=\int_{-\infty}^{\infty} \sqrt{\lambda-w} \mathrm{e}^{a(\lambda, R, \theta)} d \lambda,
\end{aligned}
$$




$$
I_{5}(R, \theta)=\int_{-\infty}^{\infty} \frac{\lambda}{\sqrt{\lambda+w}} \mathrm{e}^{a(\lambda, R, \theta)} d \lambda
$$

Where $a(\lambda, R, \theta)=-R\left(\mathrm{i} \lambda \cos \theta+|\sin \theta| \sqrt{\lambda^{2}-w^{2}}\right)$

It is clear that the residue contributions from $I_{2}$ and $I_{3}$ are $O(1)$ (w.r.t a series in $R$ ). For each integral we must integrate around the branch cut at $\lambda=-w$ which extends to infinity in the 3 rd quadrant of the complex $\lambda$ plane. Hence we can write

$$
\begin{aligned}
I_{2}(R, \theta) & =-2 \pi \sqrt{\delta+w} \mathrm{e}^{R\left(\mathrm{i} \delta \cos \theta-|\sin \theta| \sqrt{\delta^{2}-w^{2}}\right)} \\
& +2 \mathrm{e}^{\mathrm{i} w R \cos \theta+\pi \mathrm{i} / 4}\left(I_{21}(R, \theta)+\frac{(\gamma+1) M_{\infty}^{4}}{2 \beta_{\infty}^{4} w^{2}} I_{22}(R, \theta)\right), \\
I_{3}(R, \theta) & =\frac{2 \pi \delta}{\sqrt{\delta-w}} \mathrm{e}^{R\left(\mathrm{i} \delta \cos \theta-|\sin \theta| \sqrt{\delta^{2}-w^{2}}\right)} \\
& +2 \mathrm{e}^{\mathrm{i} w R \cos \theta-\pi \mathrm{i} / 4}\left(I_{31}(R, \theta)+\frac{(\gamma+1) M_{\infty}^{4}}{2 \beta_{\infty}^{4} w^{2}} I_{32}(R, \theta)\right), \\
I_{4}(R, \theta) & =2 \mathrm{e}^{\mathrm{i} w R \cos \theta-\pi \mathrm{i} / 4} I_{41}(R, \theta), \\
I_{5}(R, \theta) & =2 \mathrm{e}^{\mathrm{i} w R \cos \theta+\pi \mathrm{i} / 4} I_{51}(R, \theta),
\end{aligned}
$$

where

$$
\begin{aligned}
& I_{21}(R, \theta)=\int_{0}^{\infty} \frac{\sqrt{\rho-2 \mathrm{i} w}}{\rho+\mathrm{i}(\delta-w)} \mathrm{e}^{-\rho R \cos \theta} \sin (R|\sin \theta| \rho \sqrt{1-2 \mathrm{i} w / \rho}) d \rho \\
& I_{22}(R, \theta)=\int_{0}^{\infty}(\rho+\mathrm{i}(\delta-w)) \sqrt{\rho-2 \mathrm{i} w} \mathrm{e}^{-\rho R \cos \theta} \sin (R|\sin \theta| \rho \sqrt{1-2 \mathrm{i} w / \rho}) d \rho \\
& I_{31}(R, \theta)=\int_{0}^{\infty} \frac{\rho-\mathrm{i} w}{\sqrt{\rho}(\rho+\mathrm{i}(\delta-w))} \mathrm{e}^{-\rho R \cos \theta} \cos (R|\sin \theta| \rho \sqrt{1-2 \mathrm{i} w / \rho}) d \rho \\
& I_{32}(R, \theta)=\int_{0}^{\infty} \frac{(\rho-\mathrm{i} w)(\rho+\mathrm{i}(\delta-w))}{\sqrt{\rho}} \mathrm{e}^{-\rho R \cos \theta} \cos (R|\sin \theta| \rho \sqrt{1-2 \mathrm{i} w / \rho}) d \rho \\
& I_{41}(R, \theta)=\int_{0}^{\infty} \sqrt{\rho-2 \mathrm{i} w} \mathrm{e}^{-\rho R \cos \theta} \sin (R|\sin \theta| \rho \sqrt{1-2 \mathrm{i} w / \rho}) d \rho \\
& I_{51}(R, \theta)=\int_{0}^{\infty} \frac{(\rho-\mathrm{i} w)}{\sqrt{\rho}} \mathrm{e}^{-\rho R \cos \theta} \cos (R|\sin \theta| \rho \sqrt{1-2 \mathrm{i} w / \rho}) d \rho
\end{aligned}
$$

We retain the function $e^{-\rho R \cos \theta}$ in its unexpanded form to ease integration. For each integral we construct an additive composite expansion of the integrand for $\rho=O(1)$ and $\rho^{*}=\rho R=O(1)$, by taking the series expansions for each limit, summing them, and subtracting off the common terms. We retain only terms that give us singularities in $R$ 
when returned to equation (2.2.4.1). The resulting expansions are integrated to give

$$
\begin{aligned}
I_{21}(R, \theta) & \sim \sqrt{\pi} \frac{\sin \theta / 2}{\sqrt{R}} \\
I_{22}(R, \theta) & \sim \sqrt{\pi}\left(\frac{3 \sin 5 \theta / 2}{4 R^{5 / 2}}+\frac{\mathrm{i}(\delta-2 w) \sin 3 \theta / 2}{2 R^{3 / 2}}-\frac{3 \mathrm{i} w}{4 R^{3 / 2}} \sin \theta \cos 5 \theta / 2\right. \\
& \left.+\frac{3 w^{2}}{8 R^{1 / 2}} \sin ^{2} \theta \sin 5 \theta / 2+\frac{(2 \delta-3 w) w}{4 R^{1 / 2}} \sin \theta \cos 3 \theta / 2+\frac{(2 d-w) w}{2 R^{1 / 2}} \sin \theta / 2\right) \\
I_{31}(R, \theta) & \sim \sqrt{\pi} \frac{\cos \theta / 2}{R^{1 / 2}}, \\
I_{32}(R, \theta) & \sim \sqrt{\pi}\left(\frac{3 \cos 5 \theta / 2}{4 R^{5 / 2}}+\frac{\mathrm{i}(\delta-2 w) \cos 3 \theta / 2}{2 R^{3 / 2}}+\frac{3 \mathrm{i} w}{4 R^{3 / 2}} \sin \theta \sin 5 \theta / 2\right. \\
& \left.+\frac{3 w^{2}}{8 R^{1 / 2}} \sin ^{2} \theta \cos 5 \theta / 2-\frac{(2 \delta-3 w) w}{4 R^{1 / 2}} \sin \theta \sin 3 \theta / 2+\frac{(2 d-w) w}{2 R^{1 / 2}} \cos \theta / 2\right) \\
I_{41}(R, \theta) & \sim \sqrt{\pi}\left(\frac{1}{2 R^{3 / 2}} \sin 3 \theta / 2-\frac{\mathrm{i} w}{2 R^{1 / 2}} \sin \theta \cos 3 \theta / 2-\frac{\mathrm{i} w}{R^{1 / 2}} \sin \theta / 2\right) \\
I_{51}(R, \theta) & \sim \sqrt{\pi}\left(\frac{1}{2 R^{3 / 2}} \cos 3 \theta / 2+\frac{\mathrm{i} w}{2 R^{1 / 2}} \sin \theta \sin 3 \theta / 2-\frac{\mathrm{i} w}{R^{1 / 2}} \cos \theta / 2\right)
\end{aligned}
$$

Note we have neglected the residue contributions here as they result in non singular terms of $R$. The $R^{-1 / 2}$ terms in the above expansions are a correction to those given in Tsai (1992).

We find the asymptotic behaviour of $H_{3 p}^{*}$ for $R \ll 1$ to be

$$
H_{3 p}^{*} \sim \frac{A_{n} a \mathrm{e}^{\mathrm{i} \pi / 4}(\gamma+1) M_{\infty}^{4}}{2 \sqrt{\pi} \beta_{\infty}^{4} w^{2} \sqrt{\delta+w}}\left(\frac{\sin 2 \theta}{R^{2}}+\frac{\mathrm{i} \delta \sin \theta}{R}\right)
$$

This is clearly singular at $R=0$ which is not what we expect the solution to behave like at the nose of the aerofoil, therefore we consider a local integration of (2.2.4.1) for $R \ll 1$. This is found through the same procedure as above. In particular the integral to which we need the composite expansion is

$$
I_{1}(R, \theta)=\int_{0}^{\infty} \frac{1}{(\rho+\mathrm{i}(\delta-w)) \sqrt{\rho}} \mathrm{e}^{-\rho R \cos \theta} \sin (R|\sin \theta| \rho \sqrt{1-2 \mathrm{i} w / \rho}) d \rho
$$


since we can write

$$
H_{0}(R, \theta)=\frac{A_{n} \operatorname{sgn}(\Psi)}{\beta_{\infty} \pi \sqrt{\delta+w}}\left(\frac{\pi}{\sqrt{\delta-w}} \mathrm{e}^{R\left(\mathrm{i} \delta \cos \theta-|\sin \theta| \sqrt{\delta^{2}-w^{2}}\right)}-\mathrm{e}^{\mathrm{i} w R \cos \theta+\pi \mathrm{i} / 4} I_{1}(R, \theta)\right) .
$$

Hence

$$
H_{0} \sim \frac{2 A_{n} \mathrm{e}^{\mathrm{i} \pi / 4}}{\beta_{\infty} \sqrt{\pi} \sqrt{\delta+w}} \sqrt{R} \cos \theta / 2
$$

Given

$$
D\left(H_{3}\right)=\frac{(\gamma+1) M_{\infty}^{4} a}{\beta_{\infty}^{3}}\left(\frac{\partial^{2} H_{0}}{\partial \Psi^{2}} \frac{1}{\sqrt{R}} \sin \theta / 2+\frac{1}{2 R^{3 / 2}} \frac{\partial H_{0}}{\partial \Phi} \sin 3 \theta / 2\right)
$$

upon changing variables to

$$
\begin{aligned}
& Z=\Phi+\mathrm{i} \Psi \\
& \bar{Z}=\Phi-\mathrm{i} \Psi
\end{aligned}
$$

we find that

$$
4 \frac{\partial^{2} H_{3}}{\partial Z \partial \bar{Z}}=\frac{\mathrm{e}^{-\mathrm{i} \pi / 4} A_{n}(\gamma+1) M_{\infty}^{4} a}{4 \sqrt{\pi} \sqrt{\delta+w} \beta_{\infty}^{3}}\left(\frac{1}{Z^{2}}-\frac{1}{\bar{Z}^{2}}\right)
$$

and hence

$$
H_{3 p}^{*}=-\frac{\mathrm{e}^{\mathrm{i} \pi / 4} A_{n}(\gamma+1) M_{\infty}^{4} a}{8 \sqrt{\pi} \sqrt{\delta+w} \beta_{\infty}^{4}} \sin 2 \theta+c_{1}(Z)+c_{2}(\bar{Z}),
$$

where $c_{1,2}$ can be determined through boundary conditions. We note that these functions cannot tend to infinity as $R \rightarrow 0$, hence the behaviour of $H_{3}$ near $R=0$ is non singular. We therefore require a complementary solution to the homogeneous equation governing $H_{3}$, i.e. the Helmholtz equation, to eliminate the singularities in (A.6). This solution must also satisfy the radiation condition at infinity. We consider therefore the Hankel functions of the first kind or orders 1 and $2 ; H_{1,2}^{(1)}(w R)$, in the forms

$$
H_{2}^{(1)}(w R) \sin 2 \theta \quad \text { and } \quad H_{1}^{(1)}(w R) \sin \theta
$$

To cancel the singularities at zero we need appropriate constant multipliers. Adding this complementary solution to $H_{3 p}^{*}$ gives the non-singular particular solution

$$
H_{3 p}=H_{3 p}^{*}+\frac{A_{n} a \mathrm{e}^{\mathrm{i} \pi / 4}(\gamma+1) M_{\infty}^{4}}{2 \sqrt{\pi} \beta_{\infty}^{4} w^{2} \sqrt{\delta+w}}\left(-\frac{\pi w^{2} \mathrm{i}}{4} H_{2}^{(1)}(w R) \sin 2 \theta+\frac{\pi \delta w}{2} H_{1}^{(1)}(w R) \sin \theta\right)
$$

We now seek a complementary solution, $H_{3 c}$, that satisfies the boundary condition 
on the aerofoil, i.e.

$$
\begin{aligned}
D\left(H_{3 c}\right) & =0, \\
\left.\frac{\partial H_{3 c}}{\partial \Psi}\right|_{\substack{\Phi>0 \\
\Psi=0}} & =d(\Phi),
\end{aligned}
$$

where

$$
d(\Phi)=-\frac{A_{n} M_{\infty}^{2} a}{4 \pi \beta_{\infty}^{2} \sqrt{\delta+w}} \frac{1}{\Phi^{3 / 2}} \int_{-\infty}^{\infty} \frac{\mathrm{e}^{-\mathrm{i} \lambda \Phi}}{(\lambda+\delta) \sqrt{\lambda+w}} d \lambda-\left.\frac{\partial H_{3 p}^{*}}{\partial \Psi}\right|_{\substack{\Phi>0 \\ \Psi=0}}
$$

After some algebra and contour integration, we find

$$
\begin{aligned}
\left.\frac{\partial H_{3 p}^{*}}{\partial \Psi}\right|_{\substack{\Phi>0 \\
\Psi=0}}= & \frac{A_{n} a}{\sqrt{\pi} \sqrt{\delta+w}}\left[\frac{(\gamma+1) M_{\infty}^{4}}{2 \beta_{\infty}^{4} w^{2}} \mathrm{e}^{\mathrm{i} w \Phi+i \pi / 4}\left(\frac{2}{\Phi^{3}}+\frac{\mathrm{i}(\delta-2 w)}{\Phi^{2}}+\frac{w(\delta-w)}{\Phi}\right)\right. \\
& +\frac{\sqrt{\pi} \mathrm{e}^{\mathrm{i} \delta \Phi}}{\sqrt{\delta-w}}\left(2\left(\delta^{2}-w^{2}\right) \sqrt{\Phi}-\frac{\mathrm{i} \delta}{\sqrt{\Phi}}\right) \operatorname{erf}\left(\mathrm{e}^{\mathrm{i} \pi / 4} \sqrt{(\delta-w) \Phi}\right)-2 \mathrm{i}(\delta+w) \mathrm{e}^{\mathrm{i} w \Phi+\mathrm{i} \pi / 4} \\
& \left.-\frac{(\gamma+1) M_{\infty}^{4} \mathrm{e}^{\mathrm{i} \pi / 4} \pi w}{2 \beta_{\infty}^{4} w^{2}}\left(\frac{\mathrm{i} w}{\Phi} H_{2}^{(1)}(w \Phi)-\frac{\delta}{2 \Phi} H_{1}^{(1)}(w \Phi)\right)\right]
\end{aligned}
$$

We deduce that $H_{3 c}(\Phi, 0)=0$ for $\Phi<0$ hence we can solve (A.10) using the Wiener-Hopf method. To simplify, we set

$$
d(\Phi)=d_{1}(\Phi)+d_{2}(\Phi)+d_{3}(\Phi)
$$

where

$$
\begin{gathered}
d_{1}(\Phi)=-\frac{A_{n} a}{\sqrt{\delta^{2}-w^{2}}} \mathrm{e}^{\mathrm{i} \delta \Phi}\left(2\left(\delta^{2}-w^{2}\right) \sqrt{\Phi}-\frac{\mathrm{i} \delta}{\sqrt{\Phi}}\right) \\
d_{2}(\Phi)=\frac{A_{n} a w \mathrm{e}^{\mathrm{i} w \Phi+\mathrm{i} \pi / 4}}{\sqrt{\pi} \sqrt{\delta+w}(\delta-w)}\left(1-\frac{(\gamma+1) M_{\infty}^{4}}{2 \beta_{\infty}^{4} w^{2}}(\delta-w)^{2}\right) \frac{1}{\Phi} \\
d_{3}(\Phi)=\frac{A_{n} a \mathrm{e}^{\mathrm{i} w \Phi+\mathrm{i} \pi / 4}}{\sqrt{\pi} \sqrt{\delta+w}}\left[2 \mathrm{i}(\delta+w)-\frac{w}{(\delta-w) \Phi}-\frac{(\gamma+1) M_{\infty}^{4}}{2 \beta_{\infty}^{4} w^{2}}\left(\frac{2}{\Phi^{3}}+\frac{\mathrm{i}(\delta-2 w)}{\Phi^{2}}\right)\right. \\
+\frac{\sqrt{\pi} \mathrm{e}^{\mathrm{i}(\delta-w) \Phi-\mathrm{i} \pi / 4}}{\sqrt{\delta-w}}\left(2\left(\delta^{2}-w^{2}\right) \sqrt{\Phi}-\frac{\mathrm{i} \delta}{\sqrt{\Phi}}\right) \operatorname{erfc}\left(\mathrm{e}^{\mathrm{i} \pi / 4} \sqrt{(\delta-w) \Phi}\right) \\
+\frac{(\gamma+1) M_{\infty}^{4}}{4 \beta_{\infty}^{4} w^{2}} \pi w \mathrm{e}^{-\mathrm{i} w \Phi}\left(\frac{\mathrm{i} w}{\Phi} H_{2}^{(1)}(w \Phi)-\frac{\delta}{\Phi} H_{1}^{(1)}(w \Phi)\right) \\
\left.+\frac{A_{n} a M_{\infty}^{2} \mathrm{e}^{\mathrm{i} \delta \Phi}}{\beta_{\infty}^{2} \sqrt{\delta^{2}-w^{2}} \Phi^{3 / 2}} \operatorname{erf}\left(\mathrm{e}^{\mathrm{i} \pi / 4} \sqrt{(\delta-w) \Phi}\right)\right]
\end{gathered}
$$

We again assume that $\delta$ has a small positive imaginary part so that when we apply the 
Fourier transform, our integrals converge at infinity. We set

$$
H_{3 c}=\Sigma_{j=1}^{3} H_{3 c_{j}}
$$

where $H_{3 c_{i}}$ corresponds to boundary condition $d_{i}$. Using the Wiener-Hopf method, we find

$$
H_{3 c_{j}}=-\frac{\operatorname{sgn}(\Psi)}{2 \pi} \int_{-\infty}^{\infty} F_{+j}(\lambda) \frac{\mathrm{e}^{a(\lambda, \Phi, \Psi)}}{\sqrt{\lambda+w}} d \lambda
$$

where

$$
F_{+j}=\int_{0}^{\infty} \frac{d_{j}(x) \mathrm{e}^{\mathrm{i} \lambda x}}{\sqrt{\lambda-w}} \operatorname{erf}\left(\mathrm{e}^{\mathrm{i} \pi / 4} \sqrt{(\lambda-w) x}\right) d x .
$$

We leave $F_{+3}$ in terms of an integral but evaluate $F_{+1,2}$, noting that the formula

$$
F_{+}(\lambda)=\frac{1}{2 \pi \mathrm{i}} \int_{C_{1}} \frac{d \kappa}{\sqrt{\kappa-w}(\kappa-\lambda)} \int_{0}^{\infty} d(x) \mathrm{e}^{\mathrm{i} \kappa x} d x
$$

where the contour of integration runs parallel to, but just above, the real axis, and below $w$, is more appropriate to evaluate $F_{+1,2}$ since it allows us to properly assess the effects of the branch cuts. For $F_{+1}$ we evaluate the integral with respect to $x$ first, whereas for $F_{+2}$ is it easier to evaluate the integral with respect to $\kappa$ first (Tsai, 1992). This yields

$$
\begin{aligned}
& F_{+1}=\frac{A_{n} a \sqrt{\pi} \mathrm{e}^{-\mathrm{i} \pi / 4}}{\sqrt{\delta^{2}-w^{2}}}\left(\frac{2 \mathrm{i}(\delta-w)}{\pi(\lambda+\delta)}-\frac{w^{2}+\lambda \delta}{\sqrt{\lambda-w}(\delta+\lambda)^{3 / 2}}\left[1-\frac{2}{\pi} \arcsin \left(\frac{\sqrt{w-\lambda}}{\sqrt{w+\delta}}\right)\right]\right), \\
& F_{+2}=\frac{2 A_{n} a w \mathrm{e}^{\mathrm{i} \pi / 4}}{\sqrt{\pi} \sqrt{\delta+w}(\delta-w)}\left(1-\frac{(\gamma+1) M_{\infty}^{4}}{2 \beta_{\infty}^{4} w^{2}}(\delta-w)^{2}\right) \frac{1}{\sqrt{\lambda-w}} \log \left[\frac{\sqrt{2 w}+\mathrm{i} \sqrt{\lambda-w}}{\sqrt{\lambda+w}}\right] .
\end{aligned}
$$

Using the method of steepest descents for our integral expressions, and the limits of the Hankel functions at large argument we obtain the outer limit of our inner solutions;

$H_{3 p} \sim-\frac{A_{n} a \mathrm{e}^{\mathrm{i} \pi / 4} w \sin \theta \mathrm{e}^{\mathrm{i} w k r}}{\sqrt{\pi} \sqrt{\delta+w}(\delta-w \cos \theta)}\left(1-\frac{(\gamma+1) M_{\infty}^{4}}{2 \beta_{\infty}^{4} w^{2}}(\delta-w \cos \theta)^{2}\right)+L_{3 p}(\theta) \frac{\mathrm{e}^{\mathrm{i} w k r}}{\sqrt{k r}}+O(1 / k)$,

where

$$
L_{3 p}(\theta)=\frac{\mathrm{i} A_{n} a \sqrt{w}(\gamma+1) M_{\infty}^{4}}{2 \sqrt{2} \beta_{\infty}^{4} w^{2} \sqrt{\delta+w}}\left(\frac{w}{2} \sin 2 \theta-\delta \sin \theta\right)
$$

and

$$
H_{3 c_{i}} \sim L_{3 c_{i}}(\theta) \frac{\mathrm{e}^{\mathrm{i} w k r}}{\sqrt{k r}}+O\left(k^{-3 / 2}\right) \quad \text { for } i=1,2,3
$$


where

$$
\begin{array}{r}
L_{3 c_{1}}(\theta)=\frac{A_{n} a \sqrt{w} \operatorname{sgn}(\Psi) \cos (\theta / 2)}{\sqrt{2} \sqrt{\delta^{2}-w^{2}}(\delta-w \cos \theta)}\left[\frac{w-\delta \cos \theta}{\sqrt{\delta-w \cos \theta}}\left(1-\frac{2}{\pi} \arcsin \left[\frac{\sqrt{w(1+\cos \theta)}}{\sqrt{w+\delta}}\right]\right)\right. \\
\left.-\frac{2}{\pi}(\delta-w) \sqrt{(1+\cos \theta)}\right], \quad(\mathrm{A} .18 \mathrm{~b}) \\
L_{3 c_{2}}(\theta)=-\frac{\sqrt{2} \mathrm{i} \operatorname{sgn}(\psi) A_{n} a \sqrt{w}}{\pi \sqrt{\delta+w}(\delta-w)}\left(1-\frac{(\gamma+1) M_{\infty}^{4}}{2 \beta_{\infty}^{4} w^{2}}(\delta-w)^{2}\right) \log \left[\frac{\sqrt{2}+\sqrt{1+\cos \theta}}{\sqrt{1-\cos \theta}}\right], \\
L_{3 c_{3}}(\theta)=-\frac{\operatorname{sgn}(\psi) \mathrm{e}^{\mathrm{i} \pi / 4}}{\sqrt{2 \pi w}} \int_{0}^{\infty} \mathrm{e}^{-\mathrm{i} w \cos \theta x} \operatorname{erf}\left(\mathrm{e}^{-\mathrm{i} \pi / 4} \sqrt{w(1+\cos \theta) x}\right) d_{3}(x) d x .
\end{array}
$$

The expression for $L_{3 c_{1}}$ that we have found also varies from the solution presented in Tsai (1992). These expansions are valid provided $\theta \nrightarrow \nrightarrow 0,2 \pi$ because there $L_{3 c_{2}}$ is singular. To find an expansion that is uniformly valid for all values of $\theta$ we use Van der Waerden's method (Van der Waerden, 1952). We isolate the branch point of the singularity and integrate it separately. The integral of concern is

$$
J_{2}(\Phi, \Psi)=\int_{\infty}^{\infty} \frac{\mathrm{e}^{-R g(\lambda ; \theta)}}{\sqrt{\lambda^{2}-w^{2}}} \log \left[\frac{\sqrt{2 w}+\mathrm{i} \sqrt{\lambda-w}}{\sqrt{\lambda+w}}\right] d \lambda
$$

which arises from the calculation of $F_{+2}$, with $g(\lambda ; \theta)=-a(\lambda, R, \theta) / R$. We set $s^{2}=$ $g(\lambda ; \theta)+\mathrm{i} w$ since $g^{\prime}\left(\lambda_{0} ; \theta\right)=0 \rightarrow g\left(\lambda_{0} ; \theta\right)=-\mathrm{i} w$. This transforms our integral to

$$
J_{2}=\mathrm{e}^{\mathrm{i} w R} \int_{\infty}^{\infty} A(s) \mathrm{e}^{-R s^{2}} d s
$$

where

$$
A(s)=\frac{1}{\sqrt{\lambda(s)^{2}-w^{2}}} \frac{d \lambda}{d s} \log \left[\frac{\sqrt{2 w}+\mathrm{i} \sqrt{-\mathrm{i} \cos \theta\left(s^{2}-\mathrm{i} w\right)+s|\sin \theta| \sqrt{s^{2}-2 \mathrm{i} w}-w}}{\sqrt{-\mathrm{i} \cos \theta\left(s^{2}-\mathrm{i} w\right)+s|\sin \theta| \sqrt{s^{2}-2 \mathrm{i} w}+w}}\right] .
$$

The saddle point, $\lambda_{0}=-w \cos \theta$, has been mapped to $s=0$ and the contour of steepest descent has been mapped to the real $s$ axis. There is a logarithmic branch point at $s=s_{1}=-\mathrm{e}^{\pi \mathrm{i} / 4} \sqrt{w(1-\cos \theta)}$ which is the image of the branch point at $\lambda=-w$. This is the only concerning branch cut as the others $\left(s= \pm \mathrm{e}^{\mathrm{i} \pi / 4} \sqrt{2 w}\right)$ are taken away from the contour of integration. For small $\theta$ the point $s_{1}$ moves to the origin hence we must ensure 
we integrate around it to obtain a uniformly-valid solution. Expanding $A(s)$ about $s_{1}$ gives

$$
A(s) \sim-\frac{2 \log \left[s-s_{1}\right]}{\sqrt{s_{1}^{2}-2 \mathrm{i} w}}+\text { const. }+O\left(s-s_{1}\right)
$$

Hence the function

$$
A_{1}(s)=A(s)+\frac{2 \log \left[s-s_{1}\right]}{\sqrt{s_{1}^{2}-2 \mathrm{i} w}}
$$

is analytic at $s=0$ for all values of $\theta$. We substitute $A(s)=A_{1}(s)-\frac{2 \log \left[s-s_{1}\right]}{\sqrt{s_{1}^{2}-2 \mathrm{i} w}}$ back into (A.19) and integrate the term containing $A_{1}$ using the method of steepest descents. The second term has a change of variables to eliminate the variable $R$ from the exponential, and is left in integral form for later analysis. We obtain

$$
\begin{aligned}
J_{2} & \sim \frac{\sqrt{2 \pi} \mathrm{e}^{\mathrm{i} \pi / 4} \mathrm{e}^{\mathrm{i} w R}}{\sqrt{w R}}\left(\log \left[\frac{\sqrt{2}+\sqrt{1+\cos \theta}}{\sqrt{1-\cos \theta}}\right]+\sqrt{2}\left(\frac{\pi \mathrm{i}+2 \log [w]}{4 \sqrt{1+\cos \theta}}+\frac{\log [1-\cos \theta]}{2 \sqrt{1+\cos \theta}}\right)\right) \\
& -\frac{2 \mathrm{e}^{\mathrm{i} / 4} \mathrm{e}^{\mathrm{i} w R}}{\sqrt{w R} \sqrt{1+\cos \theta}}\left(\int_{-\infty}^{\infty} \log \left[x+\mathrm{e}^{\pi \mathrm{i} / 4} \sqrt{w(1-\cos \theta) R}\right] \mathrm{e}^{-x^{2}} d x-\frac{\sqrt{\pi}}{2} \log [R]\right) .
\end{aligned}
$$

So the uniformly-valid solution for the far-field expansion is found by replacing $L_{3 c_{2}}(\theta)$ by

$$
\begin{gathered}
L_{3 c_{2}}^{u}(r, \theta)=-\frac{\sqrt{2} \operatorname{sgn}(\psi) A_{n} a \mathrm{i} \sqrt{w}}{\pi \sqrt{\delta+w}(\delta-w)}\left(1-\frac{(\gamma+1) M_{\infty}^{4}}{2 \beta_{\infty}^{4} w^{2}}(\delta-w)^{2}\right) \\
{\left[\frac{\sqrt{2}}{\sqrt{\pi} \sqrt{1+\cos \theta}}\left(\frac{\sqrt{\pi}}{2} \log (k r)-\int_{-\infty}^{\infty} \log \left(s+\mathrm{e}^{\mathrm{i} \pi / 4} \sqrt{w(1-\cos \theta) k r}\right) \mathrm{e}^{-s^{2}} d s\right)\right.} \\
\left.\quad+\log \left(\frac{\sqrt{2}+\sqrt{1+\cos \theta}}{\sqrt{1-\cos \theta}}\right)+\frac{\log (1-\cos \theta)}{\sqrt{2} \sqrt{1+\cos \theta}}+\frac{\mathrm{i} \pi+2 \log (w)}{2 \sqrt{2} \sqrt{1+\cos \theta}}\right]
\end{gathered}
$$

This expansion is also different to the result presented by Tsai (1992). 


\section{Appendix B}

The pressure jump across the trailing edge is given by

$$
\begin{aligned}
& \left.p_{l}\right|_{\psi=0+}-\left.p_{l}\right|_{\psi=0-}=\frac{\mathrm{i}(\delta-w)}{\sqrt{k} \sqrt{2+\phi_{t}}} \mathrm{e}^{\mathrm{i} k\left(w-\delta M_{\infty}^{2}\right)\left(2+\phi_{t}+\alpha_{t}\right)+\mathrm{i} k w \epsilon V(0) \int_{0}^{\phi} q\left(r^{\prime}, 0\right) d r^{\prime}+2 \mathrm{i} k \epsilon F_{R}(-\infty)} \\
& {\left[\mathrm { e } ^ { \mathrm { i } k ( w - \delta M _ { \infty } ^ { 2 } ) \Gamma / 2 } \left\{P_{l}(0)+t \sqrt{k}\left[L_{1}(0)+L_{2}(0)+L_{3 p}(0)+L_{3 c_{1}}(0)+L_{3 c_{2}}(0)\right.\right.\right.} \\
& \left.+L_{3 c_{3}}(0)\right]+t \sqrt{k} \frac{\mathrm{i} A_{n} \sqrt{w}}{\sqrt{2} \pi \sqrt{\delta+w}(\delta-w)}\left(1-\frac{(\gamma+1) M_{\infty}^{4}}{2 \beta_{\infty}^{4} w^{2}}(\delta-w)^{2}\right)\left\{\int _ { 0 } ^ { 2 } \left[\frac{a}{\xi}\right.\right. \\
& \left.+\frac{\beta_{\infty}}{t^{\prime} \xi^{3 / 2}} \int_{0}^{\xi} \frac{\partial q^{(t)}}{\partial \theta}\left(r^{\prime}, 0\right) d r^{\prime}\right] \frac{\sqrt{2+\phi_{t}}}{\sqrt{2+\phi_{t}-\xi}} d \xi \\
& +\int_{2}^{2+\phi_{t}}\left[\frac{a}{\xi}+\frac{\beta_{\infty}}{t^{\prime} \xi^{3 / 2}} \int_{0}^{2} \frac{\partial q^{(t)}}{\partial \theta}\left(r^{\prime}, 0\right) d r^{\prime}\right] \frac{\sqrt{2+\phi_{t}}}{\sqrt{2+\phi_{t}-\xi}} d \xi \\
& \left.\left.\left.-\frac{a}{2}\left(\log \left[32 k\left(\phi_{t}+2\right) w\right]+\tilde{\gamma}-\frac{\pi \mathrm{i}}{2}\right)\right\}\right]\right\} \\
& -\left[\mathrm { e } ^ { - \mathrm { i } k ( w - \delta M _ { \infty } ^ { 2 } ) \Gamma / 2 } \left\{P_{l}(2 \pi)+t \sqrt{k}\left[L_{1}(2 \pi)+L_{2}(2 \pi)+L_{3 p}(2 \pi)+L_{3 c_{1}}(2 \pi)+L_{3 c_{2}}(2 \pi)\right.\right.\right. \\
& \left.+L_{3 c_{3}}(2 \pi)\right]-t \sqrt{k} \frac{\mathrm{i} A_{n} \sqrt{w}}{\sqrt{2} \pi \sqrt{\delta+w}(\delta-w)}\left(1-\frac{(\gamma+1) M_{\infty}^{4}}{2 \beta_{\infty}^{4} w^{2}}(\delta-w)^{2}\right)\left\{\int _ { 0 } ^ { 2 } \left[\frac{a}{\xi}\right.\right. \\
& \left.+\frac{\beta_{\infty}}{t^{\prime} \xi^{3 / 2}} \int_{0}^{\xi} \frac{\partial q^{(t)}}{\partial \theta}\left(r^{\prime}, 0\right) d r^{\prime}\right] \frac{\sqrt{2+\phi_{t}}}{\sqrt{2+\phi_{t}-\xi}} d \xi \\
& +\int_{2}^{2+\phi_{t}}\left[\frac{a}{\xi}+\frac{\beta_{\infty}}{t^{\prime} \xi^{3 / 2}} \int_{0}^{2} \frac{\partial q^{(t)}}{\partial \theta}\left(r^{\prime}, 0\right) d r^{\prime}\right] \frac{\sqrt{2+\phi_{t}}}{\sqrt{2+\phi_{t}-\xi}} d \xi \\
& \left.\left.\left.-\frac{a}{2}\left(\log \left[32 k\left(\phi_{t}+2\right) w\right]+\tilde{\gamma}-\frac{\pi \mathrm{i}}{2}\right)\right\}\right]\right\}+\sqrt{k} \frac{\mathrm{e}^{\pi \mathrm{i} / 4} \mathrm{i} \epsilon V(0)}{\sqrt{\pi w} \beta_{\infty}} \\
& \left.\times \int_{0}^{2} \frac{\xi y^{(c) \prime}(\xi)-y^{(c)}(\xi)}{\sqrt{2-\xi} \xi^{3 / 2}} d \xi\left(\mathrm{e}^{\mathrm{i} k\left(w-\delta M_{\infty}^{2}\right) \Gamma / 2} P_{l}(0)+\mathrm{e}^{-\mathrm{i} k\left(w-\delta M_{\infty}^{2}\right) \Gamma / 2} P_{l}(2 \pi)\right)\right],
\end{aligned}
$$

which we write as

$$
\left.p_{l}\right|_{\psi=0+}-\left.p_{l}\right|_{\psi=0-}=\frac{\Delta p\left(\phi_{t}\right)}{\sqrt{k}} \mathrm{e}^{\mathrm{i} k\left(w-\delta M_{\infty}^{2}\right) \phi_{t}} \mathrm{e}^{\mathrm{i} k w \epsilon V(0)\left[\int_{0}^{\phi} q d r-\int_{0}^{2} q d r\right]}
$$


The thickness-related pressure jump with respect to trailing-edge coordinates is given explicitly by

$$
\begin{aligned}
\Delta p^{(t)}( & \left.\phi_{t}\right)=\frac{\sqrt{2}}{\sqrt{2+\phi_{t}}} \Delta p^{(t)}(0)-t \sqrt{k} \frac{A_{n} \sqrt{w}}{\sqrt{2} \pi \sqrt{\delta+w}} \mathrm{e}^{\mathrm{i} k\left(w-\delta M_{\infty}^{2}\right)\left(2+\alpha_{t}\right)+2 \mathrm{i} k \epsilon F_{R}(-\infty)} \\
& \left(1-\frac{(\gamma+1) M_{\infty}^{4}}{2 \beta_{\infty}^{4} w^{2}}(\delta-w)^{2}\right)\left\{\mathrm{e}^{\mathrm{i} k\left(w-\delta M_{\infty}^{2}\right) \Gamma / 2} \mathrm{e}^{\mathrm{i} k w \epsilon \sigma_{l 1}(2,0)}\right. \\
& \left(\int_{0}^{2}\left[\frac{a}{\xi}+\frac{\beta_{\infty}}{t^{\prime} \xi^{3 / 2}} \int_{0}^{\xi} \frac{\partial q^{(t)}}{\partial \theta}\left(r^{\prime}, 0\right) d r^{\prime}\right]\left(\frac{1}{\sqrt{2+\phi_{t}-\xi}}-\frac{1}{\sqrt{2-\xi} \sqrt{2+\phi_{t}}}\right) d \xi\right. \\
& \left.-\frac{a}{\sqrt{2+\phi_{t}}} \log \left[\frac{\phi_{t}+2}{2}\right]+\int_{2}^{2+\phi_{t}}\left[\frac{a}{\xi}+\frac{\beta_{\infty}}{t^{\prime} \xi^{3 / 2}} \int_{0}^{2} \frac{\partial q^{(t)}}{\partial \theta}\left(r^{\prime}, 0\right) d r^{\prime}\right] \frac{1}{\sqrt{2+\phi_{t}-\xi}} d \xi\right) \\
& +\mathrm{e}^{-\mathrm{i} k\left(w-\delta M_{\infty}^{2}\right) \Gamma / 2} \mathrm{e}^{\mathrm{i} k w \epsilon \sigma_{l 1}(2,2 \pi)}\left(\int_{0}^{2}\left[\frac{a}{\xi}+\frac{\beta_{\infty}}{t^{\prime} \xi^{3 / 2}} \int_{0}^{\xi} \frac{\partial q^{(t)}}{\partial \theta}\left(r^{\prime}, 2 \pi\right) d r^{\prime}\right]\right. \\
& \left(\frac{1}{\sqrt{2+\phi_{t}-\xi}}-\frac{1}{\sqrt{2-\xi} \sqrt{2+\phi_{t}}}\right) d \xi-\frac{a}{\sqrt{2+\phi_{t}}} \log \left[\frac{\phi_{t}+2}{2}\right] \\
& \left.\left.+\int_{2}^{2+\phi_{t}}\left[\frac{a}{\xi}+\frac{\beta_{\infty}}{t^{\prime} \xi^{3 / 2}} \int_{0}^{2} \frac{\partial q^{(t)}}{\partial \theta}\left(r^{\prime}, 2 \pi\right) d r^{\prime}\right] \frac{1}{\sqrt{2+\phi_{t}-\xi}} d \xi\right)\right\} .
\end{aligned}
$$




\section{Chapter 3}

\section{High-Frequency Sound Generated by Gust-Aerofoil Interaction in Subsonic Shear Flow}

In this chapter we investigate the sound generated by gust-aerofoil interaction in subsonic shear flow. In the model described by Figure 0.6, it is assumed that the background steady flow upstream is uniform (which motivated the work of the previous chapters), however substantial structural elements upstream of the rotors could induce a background shear flow, hence in this chapter we investigate the sound generated by gust-aerofoil interaction in a background two-dimensional steady parallel shear flow. This is a natural, but significant, extension to Goldstein $(1978 a)$ who presented the evolution of a gust in parallel shear flow and evaluated the acoustic radiation of such a system due to a semiinfinite flat plate downstream of the initial gust. The solution obtained by Goldstein (1978a) describes the evolution of an arbitrary upstream unsteady vortical disturbance in a steady background shear flow, independently of solid boundary conditions. Such a solution can be implemented in this chapter, and will henceforth be known as the gust solution. After determining the gust solution, Goldstein (1978a) then applied the Wiener-Hopf method on a pair of integral conditions, representing zero pressure jump in the flow upstream of a semi-infinite flat plate (i.e. on $x \in(-\infty, 0), y=0$ ), and zero normal velocity on the solid boundary (along $x \in(0, \infty), y=0)$, to solve for the scattered acoustics.

To simplify our problem of investigating the sound generated by a thick, finite chordlength aerofoil, we first simplify the aerofoil geometry by transforming coordinates so that the aerofoil is mapped to a flat plate. The geometry of the aerofoil then arises as perturbation terms in the transformed governing equations rather than complicated boundary conditions. We make the transformation of variables to an orthogonal coordinate system where one coordinate axis is aligned with the streamlines of the flow. The second coordinate is known as a pseudo-velocity potential since it is everywhere orthogonal to the streamlines. We choose to work specifically with parallel shear flow in order to ensure we can make this transformation between coordinate systems. The sound 
generated by a gust interacting with semi-infinite flat plates in steady three-dimensional transverse shear flows has been investigated by Goldstein (1979), however due to the greater complexity of our choice of aerofoil, now having a finite chord length and nonzero thickness, we are constrained for the time being to parallel shear flow. Goldstein later extended his work, in Goldstein et al. (2013), to consider a vortical perturbation interacting with more general solid bodies in transverse shear flow. We draw attention to a limitation of their work in that it does not permit solid bodies with non-zero thickness since a requirement imposed is that the shear flow is everywhere parallel to the solid surfaces. Goldstein et al. (2013) is therefore more applicable to duct acoustics than to the gust-aerofoil interaction problems that we are studying.

We suppose the aerofoil is thin, with thickness parameter denoted by $\epsilon \ll 1$, and that the reduced frequency of the incident gust is high, denoted by $k \gg 1$. The preferred limit $\epsilon k=O(1)$ is imposed to correlate with the limits in Myers \& Kerschen (1997), Tsai (1992), and the previous chapters of this dissertation. We impose a further condition that the flow is relatively low speed, with typical Mach numbers being $O\left(\epsilon^{1 / 2}\right)$. These limitations still allow us to study cases of practical interest, especially since the highfrequency regime remains challenging for computational aeroacoustics. We make the transformation between Cartesian coordinates, $(x, y)$, and streamline coordinates, $(\phi, \psi)$, by using the standard orthogonal curvilinear coordinate expressions that can be found in Batchelor (1967, Appendix 2). Once the aerofoil is mapped to a flat plate in $(\phi, \psi)$ space we analyse the problem similarly to the high-frequency gust-aerofoil interaction considered in Chapter 2. We use the method of matched asymptotic expansions (Van Dyke, 1975) to split the problem into several asymptotic regions around the aerofoil (shown in Figure 3.1); local inner regions which scale as $O\left(k^{-1}\right)$ about the leading and trailing edges of the aerofoil; a transition solution which scales as $O\left(k^{-1 / 2}\right)$ along the upper and lower surfaces of the aerofoil accounting for the effects of physical curvature; an acoustic outer solution which scales as $O(1)$ and describes the propagation into the far field of the sound generated by the gust-aerofoil interaction; and a wake transition solution which scales as $O\left(k^{-1 / 2}\right)$ downstream of the aerofoil ensuring the Kutta condition is met across the wake. The solution is determined in each region, and matched to surrounding regions. We focus on the high-frequency $(k \gg 1)$ regime since in such a range, fully numerical calculations are much more difficult to carry out, although we note that the gust solution presented in Section 3.2 is valid for all frequencies (subject to a rescaling of the asymptotic series (3.2.0.6)).

In Section 3.1 we outline the coordinate transformation for a general two-dimensional parallel shear flow from Cartesian coordinates to streamline coordinates and apply it to 


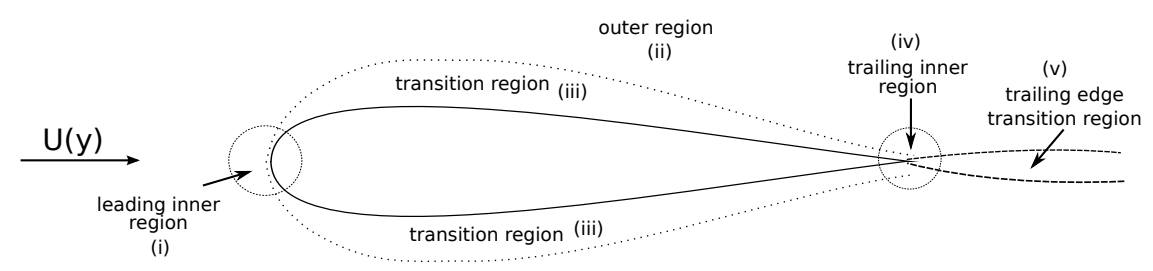

(a) In $(x, y)$ space.

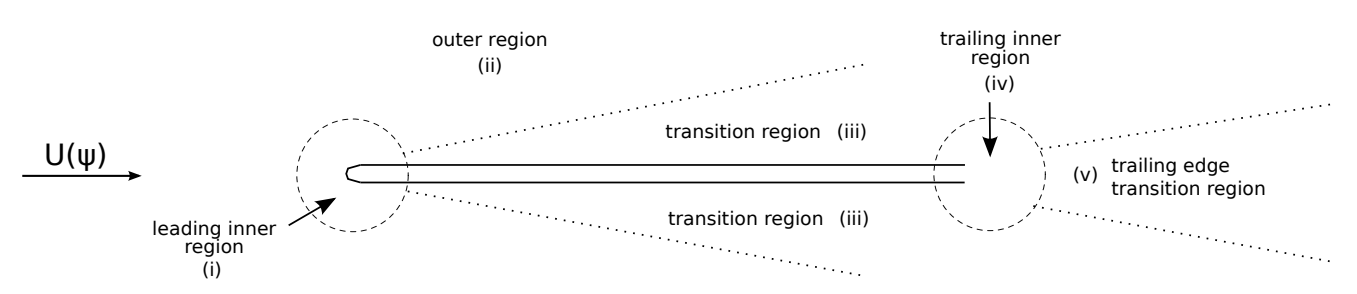

(b) In $(\phi, \psi)$ space.

Figure 3.1: Asymptotic regions around the aerofoil; leading- and trailing-edge inner regions, (i) and (iv), scale as $O\left(k^{-1}\right)$, and the width of the transition regions, (iii) scales as $O\left(k^{-1 / 2}\right)$. The outer region (ii) is $O(1)$. We solve for (i) in Section 3.3, then solve for a leading-edge contribution to (iii) in Section 3.4. Region (iii) is solved for in Section 3.5, and the trailing-edge contribution to (ii) along with regions (iv) and (v) are solved for in Section 3.6.

the linearised Euler equations governing the gust-aerofoil interaction problem. Section 3.2 outlines the method used to obtain the evolution of the gust in a shear flow; we find small thickness-related perturbation terms to Goldstein's solution because our steady flow contains thickness related perturbations due to the flow augmenting around the aerofoil. In Section 3.3 we solve the governing equations in the inner leading-edge region to obtain a solution for the acoustic pressure generated at the leading edge, correct to two orders of magnitude. Here in the inner region, the aerofoil can be assumed to be a semi-infinite flat plate extending to $+\infty$. We therefore solve the governing equations and boundary conditions (of continuous pressure upstream of the aerofoil, and zero normal velocity on the solid surface) by constructing a dual integral equation and using the Wiener-Hopf method. This is similar to Goldstein (1978a), however, due to the non-zero thickness of the aerofoil, we must find thickness-related perturbations to Goldstein's flatplate solution. The inner solution is matched to a leading-edge far-field outer solution in Section 3.4, and the transition solution to account for surface curvature along with the correction of the zero-velocity boundary condition on the solid surface is constructed in Section 3.5. The procedure is repeated at the trailing edge along with appropriate matchings in Section 3.6. In the trailing-edge inner region the aerofoil is seen as a semiinfinite flat plate extending to $-\infty$, so we construct a dual integral equation imposing 
continuity of pressure across the wake downstream of the aerofoil, and zero normal velocity on the solid surface, then solve using the Wiener-Hopf method. This is also solved to two orders of magnitude, as with the leading-edge inner solution. The trailingedge far-field outer solution is found to two orders of magnitude in the amplitude and the phase, and is matched to the trailing-edge inner solution. The trailing-edge transition solution is not required to obtain approximations for the total far-field acoustics, hence is mentioned only briefly in this section. In Section 3.8 we illustrate our results by plotting the far-field acoustic pressure generated by gust-aerofoil interaction in specified shear flows around symmetric Joukowski aerofoils. Section 3.9 contains concluding remarks.

\subsection{Formulation and Governing Equations}

We consider a thin symmetric aerofoil aligned parallel to the $x$-axis, with surface $y=$ $\pm \epsilon y(x), 0 \leqslant x \leqslant 2$. Here, lengths have been non-dimensionalised using the aerofoil semichord $b^{*}$ (* denotes dimensional quantities). The aerofoil sits in a mean shear flow of velocity $\boldsymbol{U}$, which is aligned parallel to the aerofoil chord at infinity (velocities are nondimensionalised using $U_{\infty}^{*}$, the uniform mean flow speed far upstream as $y \rightarrow \infty$, and we explicitly exclude the case $\left.U_{\infty}^{*}=0\right)$. We work in the orthogonal $(\phi, \psi)$ coordinate system, where $\psi$ is the non-dimensional mean flow streamfunction and $\phi$ is the non-dimensional pseudo-velocity potential, chosen such that surfaces of constant $\phi$ and $\psi$ are orthogonal. The origin in $(\phi, \psi)$ space is located at the leading edge of the aerofoil. This coordinate system has the advantage of mapping the aerofoil surface onto the flat plate $\psi=0$, $0 \leqslant \phi \leqslant \phi_{e}$, where $\phi_{e}$ is the location of the trailing edge and must be calculated from the mean-flow solution. Far upstream the steady shear flow velocity is $U_{0}(\psi) \boldsymbol{e}_{\phi}$, where $\boldsymbol{e}_{\phi}$ is the unit vector in the $\phi$ direction, and the shear profile $U_{0}(\psi)$ is a given function (with the property that $U_{0} \rightarrow 1$ as $\psi \rightarrow \pm \infty$ ). The presence of the thin aerofoil distorts the mean flow similarly to Chapters 1 and 2, so again we write the total mean velocity as $\left(U_{0}(\psi)+\epsilon q(\phi, \psi)\right) \boldsymbol{e}_{\phi}+O\left(\epsilon^{2}\right)$. The local Mach number is denoted by $M(\psi)$, which takes the value $M_{\infty}$ as $\psi \rightarrow \pm \infty$; in what follows we consider low Mach number flow only, with $M=O\left(\epsilon^{1 / 2}\right)$. This means that the steady flow around the aerofoil can be determined to $O(\epsilon)$ using incompressible thin-aerofoil theory, and it follows from (1.1.1.2) and Thwaites (1960) that

$$
q(z)=\frac{U_{0}(0)}{\pi} \operatorname{Re}\left[\int_{0}^{2} \frac{d y(x) / d x}{z-x} d x\right],
$$

where $z=\phi+\mathrm{i} \psi$. Furthermore, the corrections to the otherwise uniform steady pressure, density and sound speed due to the presence of the aerofoil are of $O\left(\epsilon M_{\infty}^{2}\right)=O\left(\epsilon^{2}\right)$ (see 
(1.1.2.3)), and to $O(\epsilon)$ are therefore ignored.

Let the incident gust have typical amplitude which is much less than $\epsilon$, allowing linearisation about the mean flow, and dimensional frequency $\omega^{*}$. In what follows we non-dimensionalise time using $b^{*} / U_{\infty}^{*}$, to give non-dimensional hydrodynamic frequency $\omega=\omega^{*} b^{*} / U_{\infty}^{*}$, and we also introduce the non-dimensional acoustic frequency $k=\omega M_{\infty}$, where $M_{\infty}=U_{\infty}^{*} / c_{\infty}^{*}$. We suppose $k$ is large, with preferred limit $k=O\left(\epsilon^{-1}\right)$. The unsteady velocity, pressure and density are written in the form

$$
\{u, v, p, \rho\}(\phi, \psi, t)=\{u, v, p, \rho\}(\phi, \psi) \mathrm{e}^{-\mathrm{i} \omega t}
$$

and we make one further assumption that the flow is isentropic, which means that the pressure and density fluctuations are connected by $\rho=M_{\infty}^{2} p$. The idea now is to substitute this unsteady perturbation into the equations of mass and (inviscid) momentum conservation, linearised about the steady base flow. In order to transform these equations into $(\phi, \psi)$ space, we use the well-known results for orthogonal curvilinear coordinates, see for example Batchelor (1967, Appendix 2). The metric elements, given by Finnigan (1983), for $(\phi, \psi)$-space are

$$
h_{\phi}=\zeta|\boldsymbol{U}| \quad h_{\psi}=|\boldsymbol{U}|
$$

where $\zeta$ is defined by

$$
\frac{\Omega}{|\boldsymbol{U}|^{2}}=\frac{\partial \log \zeta}{\partial \psi}
$$

and $\Omega=-\nabla_{\boldsymbol{x}}^{2} \psi$ is the mean vorticity $\left(\boldsymbol{\nabla}_{\boldsymbol{x}}\right.$ denotes the differential operator with respect to non-dimensional Cartesian coordinates). This leads to

$$
\begin{aligned}
-i \omega u+\zeta|\boldsymbol{U}|^{2} \frac{\partial u}{\partial \phi}-\Omega v+\zeta|\boldsymbol{U}| \frac{\partial p}{\partial \phi} & =-u \zeta \frac{\partial}{\partial \phi}\left(\frac{|\boldsymbol{U}|^{2}}{2}\right) \\
-\mathrm{i} \omega v+\zeta|\boldsymbol{U}|^{2} \frac{\partial v}{\partial \phi}+|\boldsymbol{U}| \frac{\partial p}{\partial \psi} & =v \zeta \frac{\partial}{\partial \phi}\left(\frac{|\boldsymbol{U}|^{2}}{2}\right)-2 v\left(\Omega+\frac{\partial}{\partial \psi}\left(\frac{|\boldsymbol{U}|^{2}}{2}\right)\right) \\
-\mathrm{i} \omega \rho+\zeta|\boldsymbol{U}| \frac{\partial u}{\partial \phi}+|\boldsymbol{U}| \frac{\partial v}{\partial \psi}+\zeta|\boldsymbol{U}|^{2} \frac{\partial \rho}{\partial \phi} & =\frac{v}{|\boldsymbol{U}|}\left(\Omega+\frac{\partial}{\partial \psi}\left(\frac{|\boldsymbol{U}|^{2}}{2}\right)\right)+u \zeta \frac{\partial|\boldsymbol{U}|}{\partial \phi}
\end{aligned}
$$

for the two momentum and one mass equations respectively.

Since we are considering the case of a parallel shear flow disturbed by a thin aerofoil, we make the expansions

$$
\zeta|\boldsymbol{U}|^{2}=U_{0}(\psi)+\epsilon N_{1}(\phi, \psi)
$$




$$
\begin{aligned}
\Omega & =-U_{0} \frac{d U_{0}}{d \psi}+\epsilon N_{2}(\phi, \psi), \\
\zeta|\boldsymbol{U}| & =1+\epsilon N_{3}(\phi, \psi),
\end{aligned}
$$

where the first term on the right in each case is the parallel flow result and $N_{1,2,3}=O(1)$ are the aerofoil corrections. We note immediately that introducing the thin aerofoil into the inviscid parallel mean flow does not alter the mean vorticity, so $N_{2} \equiv 0$. However, the remaining corrections, $N_{1,3}$, are non-zero and must be determined from the solution for the mean flow. Furthermore, note that the right hand sides of (3.1.0.4) are $O(\epsilon)$, which we denote by $\epsilon N_{4,5,6}$ respectively. This allows us to rewrite (3.1.0.4) in the form

$$
\begin{aligned}
-\mathrm{i} k u+M(\psi) \frac{\partial u}{\partial \phi}+\frac{M(\psi)}{M_{\infty}} \frac{d M}{d \psi} v+\epsilon \sigma_{1}(\phi, \psi) & =-M_{\infty} \frac{\partial p}{\partial \phi}, \\
-\mathrm{i} k v+M(\psi) \frac{\partial v}{\partial \phi}+\epsilon \sigma_{2}(\phi, \psi) & =-M(\psi) \frac{\partial p}{\partial \psi}, \\
-\mathrm{i} k M_{\infty} p+\frac{\partial u}{\partial \phi}+\frac{M(\psi)}{M_{\infty}} \frac{\partial v}{\partial \psi}+M(\psi) M_{\infty} \frac{\partial p}{\partial \phi}+\epsilon \sigma_{3}(\phi, \psi) & =0,
\end{aligned}
$$

where

$$
\begin{aligned}
& \sigma_{1}(\phi, \psi)=-M_{\infty}\left(N_{4}-N_{1} \frac{\partial u}{\partial \phi}-N_{3} \frac{\partial p}{\partial \phi}\right) \\
& \sigma_{2}(\phi, \psi)=-M_{\infty}\left(N_{5}-N_{1} \frac{\partial v}{\partial \phi}-q \frac{\partial p}{\partial \psi}\right), \\
& \sigma_{3}(\phi, \psi)=-\left(N_{6}-N_{3} \frac{\partial u}{\partial \phi}-q \frac{\partial v}{\partial \psi}-N_{1} M_{\infty}^{2} \frac{\partial p}{\partial \phi}\right) .
\end{aligned}
$$

In order to complete our formulation, we introduce the Fourier transform with respect to $\phi$,

$$
F(\alpha, \psi)=\frac{1}{2 \pi} \int_{-\infty}^{\infty} \mathrm{e}^{-\mathrm{i} \alpha \phi} f(\phi, \psi) d \phi
$$

with uppercase letters denoting transformed functions. Taking the Fourier transform of (3.1.0.6) and rearranging, we obtain a single equation for the transformed pressure in the form

$$
\frac{1}{M} \frac{\partial}{\partial \psi}\left(M \frac{\partial P}{\partial \psi}\right)+\frac{2 \alpha}{k-\alpha M} \frac{d M}{d \psi} \frac{\partial P}{\partial \psi}+\frac{M_{\infty}^{2}}{M^{2}}\left((k-\alpha M)^{2}-\alpha^{2}\right) P=\epsilon \Sigma(\alpha, \psi),
$$

where

$$
\Sigma(\alpha, \psi)=-\frac{\Sigma_{2}}{M}-\mathrm{i}(k-\alpha M) \frac{M_{\infty}}{M^{2}} \Sigma_{3}-\frac{2 \alpha d M / d \psi}{M(k-\alpha M)} \Sigma_{2}-\frac{\mathrm{i} \alpha M_{\infty}}{M^{2}} \Sigma_{1} .
$$


Here, $\Sigma_{i}$ denotes the Fourier transform of $\sigma_{i}$. Equations (3.1.0.6) and (3.1.0.9a) are key results, and the rest of this chapter is concerned with determining their solution.

\subsection{Form of the Incident Gust}

The form of the incident gust in parallel shear flow has been given by Goldstein (1978a). Our flow is weakly non-parallel, thanks to the presence of the aerofoil, and this effect appears in two ways in equation (3.1.0.6) - first, in the use of $(\phi, \psi)$ coordinates, which captures the curvature of the mean streamlines, and second in the presence of the terms $\sigma_{1,2,3}$ representing the interaction of the unsteady flow with the non-uniform mean flow. Even so, Goldstein's method and solutions can be applied in our case since they rely on solutions to the governing equations, (3.1.0.6), existing and behaving with appropriate symmetry conditions which we state below in (3.2.0.1). It is clear that even in our weakly non-parallel flow, since our perturbation terms to Goldstein's equations have the relevant symmetry conditions, his gust solution can be adapted for our use. We only briefly outline Goldstein's approach and state the key results here as full details of the method can be found in Goldstein (1978a). Although the equations we have presented already are valid for arbitrary mean shear distributions, $M(\psi)$, at this point we restrict attention to the case in which $M(\psi)$ is a symmetric function, which simplifies both the form of the gust and our subsequent acoustic calculations. We also suppose that the shear layer has a single maximum or minimum, at $\psi=0$, which limits the number of critical layers where $M(\psi)=k / \alpha$, and makes the construction of the gust solution easier.

Let a triple of Fourier transformed solutions to equations (3.1.0.6) be denoted $\boldsymbol{Z}=$ $\{P, U, V\}$. Equations (3.1.0.6) have two linearly independent solutions; one of them is denoted $\boldsymbol{Z}_{1}$ say, and we construct a second linearly independent solution, $\boldsymbol{Z}_{\text {out }}$, which has the property that it consists of only outgoing waves as $\psi \rightarrow \pm \infty$. These two solutions typically must be computed numerically for a given mean shear profile, however we will be able to find asymptotic approximations for $\boldsymbol{Z}_{\text {out }}$ which are required to obtain the scattered acoustic solution. Thanks to the symmetry of the shear layer, both solutions can be written in the form

$$
\boldsymbol{Z}_{*}(\alpha, \psi)=\left\{P_{*}(\alpha,|\psi|),(\operatorname{sgn}(\psi)) U_{*}(\alpha,|\psi|), V_{*}(\alpha,|\psi|)\right\}
$$

The gust solution is now written as

$$
\boldsymbol{\zeta}_{g}(\phi, \psi) \mathrm{e}^{-\mathrm{i} w t}=\left\{p_{g}(\phi, \psi), u_{g}(\phi, \psi), v_{g}(\phi, \psi)\right\} \mathrm{e}^{-\mathrm{i} w t},
$$


and we simply state the Goldstein (1978a) result for the transverse gust velocity $v_{g}$ here,

$$
\begin{aligned}
v_{g}= & \int_{\psi}^{\infty} \mathrm{e}^{\mathrm{i} k \phi / M(\eta)} \tilde{\Omega}(\eta)\left[\frac{V_{1}(k / M(\eta), \psi)-\gamma(\eta) V_{\text {out }}(k / M(\eta), \psi)}{U_{1}(k / M(\eta), \eta)}\right] d \eta \\
& -\int_{-\infty}^{\psi} \mathrm{e}^{\mathrm{i} k \phi / M(\eta)} \tilde{\Omega}(\eta) \gamma(\eta) \frac{V_{\text {out }}(k / M(\eta), \psi)}{U_{1}(k / M(\eta), \eta)} d \eta \quad \text { for } \quad \psi>0
\end{aligned}
$$

which is obtained by assuming the gust solution is formed by a combination of $\boldsymbol{Z}_{\text {out }}$ and $\boldsymbol{Z}_{1}$ solutions, then solving for continuity of upwash velocity and pressure on $\psi=0$. In equation (3.2.0.3) we have $\gamma(\eta)=\Gamma_{+}^{ \pm}(k / M(\eta))$ for $\eta \geq 0$ with

$$
\Gamma_{+}^{ \pm}(\alpha)=\frac{P_{1}\left(\alpha, 0_{ \pm}\right) V_{\text {out }}\left(\alpha, 0_{-}\right)-V_{1}\left(\alpha, 0_{ \pm}\right) P_{\text {out }}\left(\alpha, 0_{-}\right)}{P_{\text {out }}\left(\alpha, 0_{+}\right) V_{\text {out }}\left(\alpha, 0_{-}\right)-P_{\text {out }}\left(\alpha, 0_{-}\right) V_{\text {out }}\left(\alpha, 0_{+}\right)}
$$

and $\eta$ is a function defined as the inverse of $f(\psi)=k / M(\psi)$ (note that this is well-defined since we consider a symmetric shear flow with a single turning point at $\psi=0$ ), so

$$
\psi=\eta^{ \pm}(k / M(\psi)) \quad \text { for } \quad \psi_{<}^{>} 0
$$

In (3.2.0.3), the function $\tilde{\Omega}(\psi)$ is an arbitrary vorticity distribution that is fixed by the form of incident gust at upstream infinity. Given the symmetry of our problem we choose to work solely in the upper half plane, $\psi>0$, from this point on.

We mention that whilst the general form of the solution, (3.2.0.3), is taken directly from Goldstein's work, the actual value of the solution is different, because terms dependent on $\boldsymbol{Z}_{1}$ and $\boldsymbol{Z}_{\text {out }}$ rely on the solutions to our perturbed governing equations, rather than Goldstein's flat-plate equations. The assumptions that Goldstein (1978a) used to obtain (3.2.0.3) are consistent with our perturbed equations, which ensures we are able use this form of the gust solution.

At this point we expand the unsteady flow quantities, and their Fourier transforms, in the form

$$
f=f^{0}+\epsilon \sqrt{k} f^{1}+O(\epsilon)
$$

This choice of expansion is inspired by the work in Chapter 2 which considered aerofoils in uniform flows, and showed that the leading effect of the aerofoil shape on the amplitude of the unsteady flow is to introduce an $O(\epsilon \sqrt{k})$ correction. This effect arises from the interaction between the incident gust and the large mean-flow gradients close to the leading edge (with the flow at the leading edge being represented by an inverse squareroot singularity in thin aerofoil theory). This interaction produces the $O(\epsilon \sqrt{k})$ term both close to the leading edge and throughout the flow. We expand the gust solution 
(3.2.0.3) in the form $v_{g}(\phi, \psi)=v_{g}^{0}(\phi, \psi)+\epsilon \sqrt{k} v_{g}{ }^{1}(\phi, \psi)+O(\epsilon)$, to obtain

$$
\begin{gathered}
v_{g}^{0}(\phi, \psi)=\int_{\psi}^{\infty} \mathrm{e}^{\mathrm{i} k \phi / M(\eta)} \tilde{\Omega}(\eta)\left[\frac{V_{1}^{0}(k / M(\eta), \psi)}{U_{1}^{0}(k / M(\eta), \eta)}-\frac{\gamma^{0}(\eta) V_{\text {out }}^{0}(k / M(\eta), \psi)}{U_{1}^{0}(k / M(\eta), \eta)}\right] d \eta \\
-\int_{-\infty}^{\psi} \mathrm{e}^{\mathrm{i} k \phi / M(\eta)} \tilde{\Omega}(\eta) \frac{\gamma^{0}(\eta) V_{\text {out }}^{0}(k / M(\eta), \psi)}{U_{1}^{0}(k / M(\eta), \eta)} d \eta, \\
v_{g}^{1}(\phi, \psi)=\int_{\psi}^{\infty} \frac{\mathrm{e}^{\mathrm{i} k \phi / M(\eta)} \tilde{\Omega}(\eta)}{U_{1}^{0}(k / M(\eta), \eta)}\left[V_{1}^{1}(k / M(\eta), \psi)-\gamma^{1}(\eta) V_{\text {out }}^{0}(k / M(\eta), \psi)\right. \\
\left.-\gamma^{0}(\eta) V_{\text {out }}^{1}(k / M(\eta), \psi)-U_{1}^{1}(k / M(\eta), \eta)\left(V_{1}^{0}(k / M(\eta), \psi)-\gamma^{0}(\eta) V_{\text {out }}^{0}(k / M(\eta), \psi)\right)\right] d \eta \\
-\int_{-\infty}^{\psi} \frac{\mathrm{e}^{\mathrm{i} k \phi / M(\eta)} \tilde{\Omega}(\eta)}{U_{1}^{0}(k / M(\eta), \eta)}\left[\gamma^{1}(\eta) V_{\text {out }}^{0}(k / M(\eta), \psi)+\gamma^{0}(\eta) V_{\text {out }}^{1}(k / M(\eta), \psi)\right. \\
\left.-\gamma^{0}(\eta) V_{\text {out }}^{0}(k / M(\eta), \psi) \frac{U_{1}^{1}(k / M(\eta), \eta)}{U_{1}^{0}(k / M(\eta), \eta)}\right] d \eta,
\end{gathered}
$$

for $\psi>0$. In what follows we describe how the gust interacts with the aerofoil to generate sound, and aim to find an approximation for the far field acoustics generated by the interaction. We neglect sound in the far field that is generated purely as gust selfnoise (i.e. is generated regardless of the presence of the aerofoil or the weakly non-parallel flow).

\subsection{Leading-Edge Inner Solution}

Here we investigate the sound generated by the interaction of the gust with the leading edge of the aerofoil (region (i) in Figure 3.1). We move to a leading-edge inner coordinate system, $(\Phi, \Psi)=(k \phi, k \psi)$, recalling that $k \gg 1$ is the high-frequency parameter, and we write the scattered pressure as $p_{a}(\Phi, \Psi)=p_{a}^{0}(\Phi, \Psi)+\epsilon \sqrt{k} p_{a}^{1}(\Phi, \Psi)+O(\epsilon)$, with the suffix $_{a}$ denoting that this part of the solution contains the acoustic field generated by the gust-aerofoil interaction. The leading-order solution, $p_{a}^{0}(\Phi, \Psi)$, represents the effect of the blocking of the transverse momentum of the incident gust by the solid aerofoil surface approximated as a flat plate, while the perturbation $p_{a}^{1}(\Phi, \Psi)$ represents the effects of thickness.

In the inner region the magnitude of the perturbation to the mean velocity, $q$, is 
determined by substituting inner (polar) variables into (3.1.0.1) and expanding, to give

$$
q(R, \theta)=-\frac{\mathrm{i} \sqrt{k}}{2 \sqrt{R}} \cos \frac{\theta}{2},
$$

in the case of a Joukowski aerofoil with chord length, 2, and thickness, $\epsilon$. Similarly to Chapter 2, the perturbation to the mean flow, which is of size $O(\epsilon)$ in the outer region, has been promoted to size $O(\epsilon \sqrt{k})$ in the inner region, thanks to the presence of the inverse square-root singularity at the leading edge. This is what gives rise to the expansion (3.2.0.6). In fact, provided the shear flow does not vary rapidly on $O\left(k^{-1}\right)$ lengthscales we can treat the inner regions as if they are in purely uniform flow with Mach number $M_{0}=M(0)$.

\subsubsection{General Solution for Leading-Edge Inner Acoustic Pressure}

In this inner region it appears that the aerofoil is a semi-infinite flat plate $\Phi>0, \Psi=0$, and hence we use the Wiener-Hopf method (Noble, 1998) to solve for the leading-edge inner acoustic solution. We write the solution as

$$
\begin{aligned}
& p_{a}(\Phi, \Psi)=\operatorname{sgn}(\Psi) \int_{-\infty}^{\infty} \mathrm{e}^{\mathrm{i} \alpha \Phi / k} A(\alpha) P_{\text {out }}(\alpha,|\Psi|) d \alpha \\
& v_{a}(\Phi, \Psi)=\int_{-\infty}^{\infty} \mathrm{e}^{\mathrm{i} \alpha \Phi / k} A(\alpha) V_{\text {out }}(\alpha,|\Psi|) d \alpha
\end{aligned}
$$

demanding outgoing-wave behaviour at infinity. We enforce the boundary conditions, $v_{a}=-v_{g}$ on $\Phi>0, \Psi=0$ (in order to cancel the incident gust transverse velocity on the aerofoil surface), and continuity of pressure across $\Psi=0$ for $\Phi<0$. These two conditions lead to the integral equations

$$
\begin{array}{ll}
\int_{-\infty}^{\infty} \mathrm{e}^{\mathrm{i} \alpha \Phi / k} A(\alpha) V_{\text {out }}(\alpha, 0) d \alpha=-v_{g}(\Phi, 0) & \text { for } \quad \Phi>0 \\
\int_{-\infty}^{\infty} \mathrm{e}^{\mathrm{i} \alpha \Phi / k} A(\alpha) P_{\text {out }}(\alpha, 0) d \alpha=0 & \text { for } \quad \Phi<0 .
\end{array}
$$

The solution to this Wiener-Hopf problem is described in detail in Appendix A, and we write

$$
p_{a}^{0,1}(\Phi, \Psi)=\int_{0}^{\infty} p_{a}^{0,1}(\Phi, \Psi \mid \eta) d \eta
$$


where

$$
p_{a}^{0}(\Phi, \Psi \mid \eta)=\operatorname{sgn}(\Psi) \frac{\tilde{\Omega}(\eta) \tilde{Q}^{0}(\eta) M(\eta)}{2 \pi \mathrm{i}} \int_{-\infty}^{\infty} \frac{\mathrm{e}^{\mathrm{i} \alpha \Phi / k} \kappa^{0}(k / M(\eta))_{+} P_{\text {out }}^{0}(\alpha,|\Psi| / k)}{(k-\alpha M(\eta)) \kappa^{0}(\alpha)_{+} V_{\text {out }}^{0}\left(\alpha, 0_{+}\right)} d \alpha
$$

$$
\begin{aligned}
& p_{a}^{1}(\Phi, \Psi \mid \eta)=\operatorname{sgn}(\Psi) \frac{\tilde{\Omega}(\eta) \tilde{Q}^{0}(\eta) M(\eta)}{2 \pi \mathrm{i}} \int_{-\infty}^{\infty} \frac{\mathrm{e}^{\mathrm{i} \alpha \Phi / k} \kappa^{0}(k / M(\eta))_{+} P_{\text {out }}^{1}(\alpha,|\Psi| / k)}{(k-\alpha M(\eta)) \kappa^{0}(\alpha)_{+} V_{\text {out }}^{0}\left(\alpha, 0_{+}\right)} d \alpha \\
& +\operatorname{sgn}(\Psi) \frac{\tilde{\Omega}(\eta) \tilde{Q}^{1}(\eta) M(\eta)}{2 \pi \mathrm{i}} \int_{-\infty}^{\infty} \frac{\mathrm{e}^{\mathrm{i} \alpha \Phi / k} P_{\text {out }}^{0}(\alpha,|\Psi| / k) \kappa^{0}(k / M(\eta))_{+}}{(k-\alpha M(\eta)) \kappa^{0}(\alpha)_{+} V_{\text {out }}^{0}\left(\alpha, 0_{+}\right)} d \alpha \\
& -\operatorname{sgn}(\Psi) \frac{\tilde{\Omega}(\eta) \tilde{Q}^{0}(\eta) M(\eta)}{2 \pi \mathrm{i}} \int_{-\infty}^{\infty} \frac{\mathrm{e}^{\mathrm{i} \alpha \Phi / k} P_{\text {out }}^{0}(\alpha,|\Psi| / k) \kappa^{0}(k / M(\eta))_{+}}{(k-\alpha M(\eta)) \kappa^{0}(\alpha)_{+} V_{\text {out }}^{0}\left(\alpha, 0_{+}\right)} \frac{P_{\text {out }}^{1}(\alpha, 0)}{P_{\text {out }}^{0}(\alpha, 0)} d \alpha \\
& +\operatorname{sgn}(\Psi) \frac{k M^{\prime}(\eta) G^{0}(k / M(\eta))_{-}}{2 \pi \mathrm{i} M(\eta)}\left(\frac{P_{\text {out }}^{1}(k / M(\eta), 0)}{P_{\text {out }}^{0}(k / M(\eta), 0)}-\frac{V_{\text {out }}^{1}(k / M(\eta), 0)}{V_{\text {out }}^{0}(k / M(\eta), 0)}\right) \\
& \int_{-\infty}^{\infty} \frac{\mathrm{e}^{\mathrm{i} \alpha \Phi / k} P_{\text {out }}^{0}(\alpha,|\Psi| / k)}{(k-\alpha M(\eta)) \kappa^{0}(\alpha)_{+} V_{\text {out }}^{0}\left(\alpha, 0_{+}\right)} d \alpha .
\end{aligned}
$$

The term $p_{a}^{0}(\Phi, \Psi \mid \eta)$ arises from the blocking of the transverse gust momentum by the solid body approximated as a flat plate, whilst the terms in $p_{a}^{1}(\Phi, \Psi \mid \eta)$ arise from the effects of thickness; the first term in (3.3.1.4b) occurs due to the gust interacting with the steady perturbation flow around the nose of the aerofoil, the second term arises from the blocking of the chord-wise gust momentum, and the third and final terms arise from the distortion of the sound generated at the leading edge, $p_{a}^{0}(\Phi, \Psi \mid \eta)$, by the distorted steady flow around the nose of the aerofoil. The terms $\tilde{Q}^{0,1}, G^{0}$ and $\kappa^{0}$ are defined in Appendix A. We have therefore found the first two terms in the inner region.

We see from (3.3.1.3) that to obtain the acoustic pressure, we integrate (3.3.1.4) over all values of $\eta$. We note, however, that each term in (3.3.1.4) contains the upstream vorticity distribution of the incident gust, $\tilde{\Omega}$, (for the final term in (3.3.1.4b) it is contained within $\left.G^{0}\right)$. If this is sharply peaked at a given value of $\eta$, such as if $\tilde{\Omega} \sim \mathrm{e}^{-k\left(\eta-\eta_{0}\right)^{2}}$, the dominant contribution to $p_{a}$ would occur at $\eta_{0}$ hence by Laplace's method, $p_{a}(\Phi, \Psi)$ would be proportional to $p_{a}\left(\Phi, \Psi \mid \eta_{0}\right)$. Sharply peaked vorticity distributions are typical in turbulent shear layers where the turbulent energy and vorticity are concentrated in narrow regions (see Goldstein (1978a) for further details). When explicitly evaluating the acoustic pressure for a specified shear flow in Section 3.8, we assume that the vorticity distribution of the gust is sharply peaked in such a way that we can approximate the behaviour of $p_{a}(\Phi, \Psi)$ by $p_{a}(\Phi, \Psi \mid 0)$ (the true acoustic solution comprises of $p_{a}(\Phi, \Psi \mid 0)$, multiplied by a term that depends on the exponent of the vorticity distribution, which 
would be obtained by applying Laplace's method to (3.3.1.3)). A suitable vorticity distribution in this case would be $\tilde{\Omega} \sim \mathrm{e}^{-k \eta^{2}}$, but many other distributions are possible.

\subsubsection{Outer limit of the inner solution}

\section{Solution for $p_{a}^{0}$}

Taking (3.1.0.9a) to $O(1)$ gives an equation for the Fourier transform of the leading-order pressure, $P^{0}$

$$
\frac{\partial^{2} P^{0}}{\partial \psi^{2}}-\frac{2 M^{\prime} \alpha}{\alpha M-k} \frac{\partial P^{0}}{\partial \psi}+\frac{M^{\prime}}{M} \frac{\partial P^{0}}{\partial \psi}+\frac{M_{\infty}^{2}}{M^{2}}\left[(\alpha M-k)^{2}-\alpha^{2}\right] P^{0}=0
$$

All the terms in (3.3.2.1) balance provided $\alpha=O(k)$, and we therefore define $\beta \equiv \alpha / k$ with $\beta=O(1)$. This is a valid scaling of $\alpha$ in the inner region, since the Fourier phase, $-\mathrm{i} \phi \alpha$, can then be written as $-\mathrm{i} \Phi \beta$ in inner variables, allowing for $O(1)$ variations in $\Phi$ to be analysed. In inner coordinates, to leading order, (3.3.2.1) becomes

$$
\frac{\partial^{2} P^{0}}{\partial \Psi^{2}}+\frac{M_{\infty}^{2}}{M_{0}^{2}}\left[\left(\beta M_{0}-1\right)^{2}-\beta^{2}\right] P^{0}=0
$$

Equation (3.3.2.2) has an outgoing-wave solution

$$
P_{\text {out }}^{0}(\alpha, \Psi)=C^{0}(\alpha) \exp \left[\mathrm{i} \sqrt{\left(1-\alpha M_{0} / k\right)^{2}-(\alpha / k)^{2}} \frac{M_{\infty}}{M_{0}}|\Psi|\right],
$$

where

$$
C^{0}(\alpha)=c\left[\left(1-\frac{\alpha M_{0}}{k}\right)^{2}-\left(\frac{\alpha}{k}\right)^{2}\right]^{-1 / 4}
$$

and $c$ is an arbitrary constant. The reasons we choose the factor (3.3.2.4) are twofold. First, the factor is included in order to match with the form of solution used by Goldstein (1978a) in parallel shear flow - Goldstein developed a WKB solution and the factor appears there as the usual WKB amplitude. Second, the factor is included in order to recover the leading-order directivity known to be present in leading-edge scattering of both vorticity and sound - we return to this point later in this subsection.

Taking the form of solution (3.3.2.4), substituting into (3.3.1.4) and using the method of stationary phase (Bender \& Orszag, 1978), we find that the outer limit of the leading- 
order inner acoustic solution is

$$
p_{a}^{0}(r, \theta \mid \eta) \sim-\left(\frac{\mathrm{i}}{2 \pi k r}\right)^{1 / 2} \frac{\sin \theta \mathrm{e}^{\mathrm{i} k r \lambda_{0}(\theta)}}{\left(1-M_{0}^{2} \sin ^{2} \theta\right)^{3 / 4}} \frac{\tilde{\Omega}(\eta) \tilde{Q}^{0}(\eta) M(\eta)}{1-\beta_{0} M(\eta)} \frac{\kappa^{0}(k / M(\eta))_{+} C^{0}\left(k \beta_{0}\right)}{\kappa^{0}\left(k \beta_{0}\right)_{+} V_{\text {out }}^{0}\left(k \beta_{0}, 0\right)},
$$

where $(r, \theta)$ are polar coordinates in $(\phi, \psi)$-space centred on the leading edge, and $\kappa^{0}(\alpha)_{+}$, defined in Appendix A, is

$$
\kappa^{0}(\alpha)_{+}=\left(k+\alpha\left(1-M_{0}\right)\right)^{-1 / 2}
$$

We note that the arbitrary constant, $c$ from (3.3.2.4), cancels in the term $C^{0} / V_{\text {out }}^{0}$ because of the relationship between $P$ and $V$. In (3.3.2.5) we have introduced the phase function, $\lambda$, defined as

$$
\lambda(\beta, M)=\beta \cos \theta+\frac{M_{\infty}}{M} \sqrt{(1-M \beta)^{2}-\beta^{2}},
$$

which has a point of stationary phase at

$$
\beta^{s}(M)=-\frac{1}{1-M^{2}}\left(M-\frac{\cos \theta}{\sqrt{\cos ^{2} \theta+\frac{M_{\infty}^{2}}{M^{2}} \sin ^{2} \theta-M_{\infty}^{2} \sin ^{2} \theta}}\right)
$$

The functions $\lambda_{0}(\theta)$ and $\beta_{0}(\theta)$ are defined as

$$
\lambda_{0}(\theta)=\lambda\left(\beta_{0}, M_{0}\right), \quad \beta_{0}=\beta^{s}\left(M_{0}\right) .
$$

The steady Mach number takes the value $M_{0}$ throughout the inner region, and the phase in (3.3.2.5) is therefore given by (3.3.2.7) and (3.3.2.8) with $M=M_{0}$. The function $\tilde{Q}^{0}(\eta)$ appears in the form of the incident gust and is defined following equation (A.14).

We now return to the question of the choice of the factor in (3.3.2.4). We know from Chapters 1 and 2 that the leading-order outer solution for sound-aerofoil and gust-aerofoil interaction in uniform steady flow has directivity $\cos \theta / 2$. In steady shear flow, sound is produced at the leading edge both by the scattering of the vortical gust (as in Chapter 2 ), and by the scattering of the gust self-noise (similar to Chapter 1). Throughout the inner region the mean shear does not appear, and the mean flow is simply uniform with Mach number $M_{0}$, and the $\cos \theta / 2$ directivity must therefore be recovered in the outer limit of the inner solution in the present problem too. In fact, our choice of $C^{0}(\alpha)$ gives

$$
P^{0} \propto \cos (\theta / 2)\left(1-M_{0} / 2+(3+\cos 2 \theta) M_{0}^{2} / 4+O\left(M_{0}^{3}\right)\right)
$$


confirming the required directivity to the orders of magnitude retained thus far in our calculations.

\section{Solution for $p_{a}^{1}$}

Taking $O(\epsilon \sqrt{k})$ terms in (3.1.0.9a) and converting to inner coordinates gives an equation for $P^{1}$;

$$
\frac{\partial^{2} P^{1}}{\partial \Psi^{2}}+\frac{M_{\infty}^{2}}{M_{0}^{2}}\left(1-2 M_{0} \beta-\beta^{2}\left(1-M_{0}^{2}\right)\right) P^{1}=\Sigma(\alpha, \Psi) .
$$

Here we have used the fact that to leading order $\Sigma(\alpha, \Psi) \equiv \sqrt{k} \Sigma(\alpha, k \psi)$, which follows from the inverse square-root singularity of the steady flow at the leading edge.

We solve (3.3.2.11) using the Green's function

$$
G\left(\Psi, \Psi^{\prime}\right)=\frac{M_{0}}{2 \mathrm{i} M_{\infty} \sqrt{1-2 M_{0} \beta-\beta^{2}\left(1-M_{0}^{2}\right)}} \mathrm{e}^{\mathrm{i} \sqrt{\left(1-M_{0} \beta\right)^{2}-\beta^{2}} \frac{M_{\infty}}{M_{0}}\left|\Psi-\Psi^{\prime}\right|},
$$

which represents the desired outgoing wave field, to yield

$$
P_{\text {out }}^{1}(k \beta, \Psi)=\int_{0}^{\infty} \frac{M_{0} \mathrm{e}^{\mathrm{i} \sqrt{\left(1-M_{0} \beta\right)^{2}-\beta^{2}} \frac{M_{\infty}}{M_{0}}\left|\Psi-\Psi^{\prime}\right|}}{2 \mathrm{i} M_{\infty} \sqrt{\left(1-M_{0} \beta\right)^{2}-\beta^{2}}} \Sigma\left(k \beta, \Psi^{\prime}\right) d \Psi^{\prime}+C^{1}(\beta) \mathrm{e}^{\mathrm{i} \sqrt{\left(1-M_{0} \beta\right)^{2}-\beta^{2}} \frac{M_{\infty}}{M_{0}}|\Psi|} .
$$

From (3.1.0.9b) we know that each term in $\Sigma(\alpha, \Psi)$ has a phase function

$\sqrt{\left(1-M_{0} \beta\right)^{2}-\beta^{2}} \frac{M_{\infty}}{M_{0}}|\Psi|$ (since each $\sigma_{i}$ is proportional to a linear combination of $u^{0}, v^{0}$, and $p^{0}$ ), and further, since $Q$ is symmetric and $\Omega$ is antisymmetric with respect to $\psi$, we know that $\Sigma$ is symmetric with respect to $\psi$. Setting

$$
\hat{\Sigma}(\alpha, \Psi)=\Sigma(\alpha, \Psi) \mathrm{e}^{-\mathrm{i} \sqrt{\left(1-M_{0} \beta\right)^{2}-\beta^{2}} \frac{M_{\infty}}{M_{0}}|\Psi|}
$$

so that $\hat{\Sigma}$ is phase-less in the variable $\Psi$, and completing the $\Psi^{\prime}$ integral in (3.3.2.13), we find that the outer limit of the inner solution is

$$
P_{\text {out }}^{1}(k \beta, k \psi) \sim\left(d(k \beta, k \psi)+C^{1}(k \beta)\right) \mathrm{e}^{\mathrm{i} \sqrt{\left(1-M_{0} \beta\right)^{2}-\beta^{2}} k \frac{M \infty}{M_{0}}|\psi|},
$$

where $d(k \beta, k \psi)$ is given by

$$
d(k \beta, k \psi)=\frac{\mathrm{i} \hat{\Sigma}(k \beta, k \psi) M_{0}}{k\left(\left(1-M_{0} \beta\right)^{2}-\beta^{2}\right) M_{\infty}} .
$$

Following the same arguments as in the previous subsection, we choose $C^{1}(k \beta)=C^{0}(k \beta)$, 
and then repeating the method of stationary phase to invert the Fourier transform yields an outer expansion for $p_{a}^{1}$ in the form

$$
\begin{gathered}
p_{a}^{1}(r, \theta \mid \eta) \sim-\left(\frac{\mathrm{i}}{2 \pi k r}\right)^{1 / 2} \frac{\sin \theta \mathrm{e}^{\mathrm{i} k r \lambda_{0}(\theta)}}{\left(1-M_{0}^{2} \sin ^{2} \theta\right)^{3 / 4}} \frac{\tilde{\Omega}(\eta) \tilde{Q}^{0}(\eta) M(\eta)}{1-\beta_{0} M(\eta)} \frac{\kappa^{0}(k / M(\eta))_{+} C^{0}\left(k \beta_{0}\right)}{\kappa^{0}\left(k \beta_{0}\right)_{+} V_{\text {out }}^{0}\left(k \beta_{0}, 0\right)} \\
{\left[\frac{\tilde{Q}^{1}(\eta)}{\tilde{Q}^{0}(\eta)}+M^{\prime}(\eta)\left(\frac{P_{\text {out }}^{1}(k / M(\eta), 0)}{P_{\text {out }}^{0}(k / M(\eta), 0)}-\frac{V_{\text {out }}^{1}(k / M(\eta), 0)}{V_{\text {out }}^{0}(k / M(\eta), 0)}\right)+\frac{\left(d\left(k \beta_{0}, k \psi\right)-d\left(k \beta_{0}, 0\right)\right)}{C^{0}\left(k \beta_{0}\right)}\right],}
\end{gathered}
$$

where $\beta_{0}$ and $\lambda_{0}$ are given in (3.3.2.9).

Note that whilst $\Sigma$, as defined in (3.1.0.9b), has appeared in our solution through equation (3.3.2.16), we only need to calculate the inner limit of $\Sigma$ in order to establish (3.3.2.17). This is in exact parallel to the work in Chapter 2, where we found that in a uniform flow the leading-order contribution of the volume terms only appears close to the leading edge where the mean flow gradients are large. Therefore, to calculate the outer limit of the inner leading-edge solution we only need to find the correction terms, $N_{i}$ for $i=1, . ., 6$ appearing in equation (3.1.0.6), close to the aerofoil. We first note that, since $U=U_{0}(\psi)+\epsilon q(\phi, \psi)$, we have

$$
N_{1}=q+U_{0} N_{3}
$$

while by using (3.1.0.3) we obtain the relation

$$
\frac{q}{U_{0}^{2}} \frac{d U_{0}}{d \psi}+\frac{\partial q}{\partial \psi} \frac{1}{U_{0}}=\frac{\partial N_{3}}{\partial \psi}
$$

In the leading-edge inner region, equation (3.3.2.19) can be integrated to yield

$$
N_{3}=\frac{q}{U_{0}(0)}
$$

where an arbitrary function of $\phi$ has been set to zero to ensure consistency with $N_{2}=0$. It therefore follows that $N_{1}=2 q$. The quantities $N_{4,5,6}$ can be found immediately from expressions we have obtained for $\zeta, q$, and $\Omega$, with

$$
N_{4}=u^{0} \frac{\partial q}{\partial \phi}, \quad N_{5}=v^{0} \frac{\partial q}{\partial \phi}\left(1-2 U_{0}(0)\right), \quad N_{6}=\frac{\partial q}{\partial \phi}\left(v^{0}+\frac{u^{0}}{U_{0}(0)}\right)
$$

again all evaluated in the inner region.

In summary, we have determined the first two terms in the outer limit of the inner 
pressure field, given by equations (3.3.2.5) and (3.3.2.17) which are integrated in (3.3.1.3). As mentioned previously, we assume the vorticity distribution is sharply peaked at $\eta=0$ allowing us to evaluate (3.3.1.3) using Laplace's method. We write the sum of these two (integrated) terms in the form

$$
\frac{\mathcal{D}(\theta)}{\sqrt{k r}} \exp \left(\mathrm{i} k r \lambda\left(\beta_{0}\right)\right)
$$

and we match this expression onto the outer solution in the next section. We do not explicitly define the vorticity distribution yet, and hence can only evaluate $\mathcal{D}$ up to a constant that depends on the leading-order term of

$$
\int_{0}^{\infty} \tilde{\Omega}(\eta) d \eta .
$$

The $\theta$-dependence of $\mathcal{D}$ arises from $\left(p_{a}^{0}(r, \theta \mid 0)+\epsilon \sqrt{k} p_{a}^{1}(r, \theta \mid 0)\right) \sqrt{k r} \mathrm{e}^{-\mathrm{i} k r \lambda_{0}(\theta)}$.

\subsection{Leading-Edge Outer Solution}

Before proceeding with the leading-edge outer solution, which we define to mean the outer acoustic scattered solution that is seen to emanate from the leading edge of the aerofoil, we discuss a contribution to the overall outer solution, denoted by a subscript $p$, generated by the solid surface boundary condition,

$$
v_{p}(\phi, 0)=-v_{g}(\phi, 0)
$$

for $0<\phi<\phi_{e}$. This outer solution is generated by two sources; non-acoustic gust velocities, and gust velocities associated to the self-noise. For those velocities associated to the non-acoustic part, the solution assimilates that found in gust-aerofoil interaction for uniform flow, from Section 2.3, which is an exponentially decaying hydrodynamic contribution, which propagates no sound to the far field. For the self-noise part, we recall the symmetry of our solutions, and in particular the symmetry of the normal gust velocity, which is $\operatorname{sgn}(\psi) v_{g}(\phi,|\psi|)$. Upon interaction with the solid surface, any self-noise above the aerofoil is reflected. Due to the symmetry of our problem, this reflected sound is identical to the noise we would have experienced in $\psi>0$ from self-noise generated in $\psi<0$ travelling to $\psi>0$ in the absence of the aerofoil. Given we are interested in the sound generated by the gust interacting with the aerofoil, we neglect the $v_{p}$ solution as it only contains knowledge of sound generated by the gust interacting with the shear 
flow and not specifically with the aerofoil.

In the rest of this section we are therefore concerned with determining the leadingedge contribution to the scattered pressure in the far field. The sound generated by the gust-aerofoil interaction in the leading-edge inner region, as determined in the previous section, propagates through the outer region, denoted by region (ii) in Figure 3.1, and is distorted by the mean shear. We have shown in Chapters 1 and 2 that the leadingand trailing-edge noise sources are seen as acoustic point sources by an observer in the far field. We also know that the acoustic field of a given point source in mean shear has been determined by Durbin (1983), therefore we use those results here to determine the evolution of the inner solution as it propagates through the shear flow

The outer solution which matches with the outer limit of the inner solution takes the form

$$
p_{l}=\frac{s(r, \theta) \mathcal{D}(\theta)}{\sqrt{k r}} \exp \left(\mathrm{i} k \varrho^{0}+\mathrm{i} k \epsilon \varrho^{1}\right) .
$$

Here $\mathcal{D}(\theta)$ is the directivity of the inner solution as it emerges into the outer region, as defined in (3.3.2.22). The factor $s(r, \theta)$ is the scaling factor derived by Durbin (1983) to account for the distortion of the pressure amplitude due to variation in the ray tube area through the shear, and is given by

$$
s(r, \theta)=\left(\frac{1-M_{0}^{2}}{1-M_{0}^{2} \sin ^{2} \theta}\right)^{1 / 4}\left(\frac{M-M \frac{\partial \varrho^{0}}{\partial \phi}}{M_{0}-\left.M_{0} \frac{\partial \varrho^{0}}{\partial \phi}\right|_{r \rightarrow 0}}\right)\left(\lambda \sqrt{1-M_{0}^{2}} \cos \mu^{\prime} \frac{\partial \mu^{\prime}}{\partial \mu}\right)^{-1 / 2}
$$

In (3.4.0.3), $\mu$ is the local ray angle (and $\mu^{\prime}$ is its value at the leading edge) - see Durbin (1983) equation (26b) - while $\lambda$ is the local ray speed - see Durbin (1983), following his equation (16). A factor in $s(r, \theta)$ involving the local sound speed, present in Durbin (1983), has been set to unity for our low Mach number flow. Note that $s(r, \theta) \rightarrow 1$ as $r \rightarrow 0$, while in the limit $r \rightarrow \infty, s(r, \theta) \rightarrow s(\theta)$, where the latter can be calculated from (3.4.0.3).

We determine the first two phase terms, $\varrho^{0,1}$, in (3.4.0.2) by substituting the ansatz (3.4.0.2) into an equation formed by rearranging (3.1.0.6) into a single equation for $p$. We then take the real parts of the resulting equation at the first two asymptotic orders to form two eikonal equations for $\varrho^{0,1}$. In what follows we only require the acoustic pressure in the far field (i.e. $r \rightarrow \infty$ ), and we therefore write down expressions for the phase terms which are valid there. The first eikonal equation, assuming $\varrho^{0}=r f(\theta)$, is 


$$
\begin{aligned}
4 M \sin \theta f^{\prime}(\theta)+f(\theta)^{2}\left(-2+M^{2}+M^{2} \cos 2 \theta\right) & +2+2\left(-1+M^{2} \sin ^{2} \theta\right) f^{\prime}(\theta)^{2} \\
& -4 M \cos \theta f(\theta)\left(1+M f^{\prime}(\theta) \sin \theta\right)=0,
\end{aligned}
$$

which can be solved in successive powers of $M$ to give

$$
\varrho^{0}=r \lambda\left(\beta_{\infty}, M_{\infty}\right) \equiv r \lambda_{\infty}(\theta)
$$

correct to (and including) $O\left(M^{2}\right)$, where $\beta_{\infty}=\beta^{s}\left(M_{\infty}\right)$.

The second eikonal equation is more complicated, however, since it includes contributions from the terms $\sigma_{1,2,3}$ in (3.1.0.6), which arise from the interaction between the leading-order unsteady flow and the steady-flow non-uniformity caused by the presence of the aerofoil. After some algebra we find that the second eikonal equation is

$$
\frac{\partial \varrho^{1}}{\partial \phi}+\frac{\partial \varrho^{1}}{\partial \psi}=\frac{1}{2} L(\phi, \psi)
$$

where the term $L(\phi, \psi)$ involves the terms $\sigma_{1,2,3}$. Specifically, we introduce the quantity

$$
M_{\infty} \sigma(\phi, \psi)=-\mathrm{i} k \sigma_{3}+M \frac{\partial \sigma_{3}}{\partial \phi}-\frac{\partial \sigma_{1}}{\partial \phi}-\frac{M}{M_{\infty}} \frac{\partial \sigma_{2}}{\partial \psi}
$$

which, in the light of (3.4.0.2), to leading-order in the outer region takes the form

$$
\sigma(\phi, \psi)=\frac{k^{3 / 2} L(\phi, \psi) \mathcal{D}(\theta) s(\theta)}{\sqrt{r}} \mathrm{e}^{\mathrm{i} k \varrho^{0}(r, \theta)+i k \epsilon \varrho^{1}(r, \theta)},
$$

where

$$
L(\phi, \psi)=\left(\frac{\partial \varrho^{0}}{\partial \phi}\right)^{2}\left[\frac{q}{U_{0}}+\int_{\psi}^{\infty} \frac{2 q U_{0}^{\prime}\left(\psi^{\prime}\right)}{U_{0}\left(\psi^{\prime}\right)^{2}} d \psi^{\prime}\right]+q\left(\frac{\partial \varrho^{0}}{\partial \psi}\right)^{2} .
$$

The solution of (3.4.0.6) can now be determined using the method of characteristics in the form

$$
\varrho^{1}(r, \theta)=\frac{1}{2} \int_{0}^{\phi+\psi} L(\chi, \psi) d \chi
$$

where $\chi=\phi+\psi$ is the characteristic variable.

We have therefore completed the construction of the far-field solution for the noise emanating from the leading edge of the aerofoil, and we write the acoustic pressure in the form

$$
\frac{D_{l}(\theta)}{\sqrt{k r}} \exp \left(\mathrm{i} k r \lambda_{\infty}(\theta)+\frac{1}{2} \mathrm{i} k \epsilon \int_{0}^{\phi+\psi} L(\chi, \psi) d \chi\right) .
$$


We emphasise that this solution is not valid in the mid field, where the mean flow is sheared; it is only valid in the far field, where $M \approx M_{\infty}$. This excludes small angles, of size $O(1 / r)$, close to the downstream direction, $\theta=0$.

\subsection{Leading-Edge Transition Solution}

The transition solution (region (iii) in Figure 3.1) accounts for the curvature of the surface of the aerofoil, in a very similar manner to the case of uniform flow considered in Chapter 2, and corrects for the boundary condition of zero normal velocity on the aerofoil surface that is violated by the leading-edge outer solution. We therefore suppose the transition solution takes the form

$$
p_{l t r}=\epsilon G(\phi, \xi) \mathrm{e}^{\frac{\mathrm{i} k \phi}{1+M}+\frac{1}{2} \mathrm{i} k \epsilon \int_{0}^{\phi} L(\phi, 0) d \phi},
$$

where $\xi=\sqrt{k} \psi$ is the transition-region coordinate above the aerofoil in the direction normal to the surface. The choice of phase in (3.5.0.1) arises from taking $\theta=0$ (equivalently $\psi=0)$ in (3.4.0.5) and (3.4.0.10).

In the transition region the leading-order expansion of (3.1.0.6) tells us that $G(\phi, \xi)$ must satisfy

$$
\frac{M^{2}}{M_{\infty}^{2}} \frac{\partial^{2} G}{\partial \xi^{2}}+2 \mathrm{i} \frac{\partial G}{\partial \phi}=0
$$

subject to the boundary condition

$$
-\left.\frac{\epsilon \mathrm{i} M}{\sqrt{k}(1+M)} \frac{\partial G}{\partial \xi}\right|_{\xi=0} \mathrm{e}^{\frac{\mathrm{i} k \phi}{1+M}+\frac{1}{2} \mathrm{i} k \epsilon \int_{0}^{\phi} L(\phi, 0) d \phi}=-\left.v_{l}^{0}\right|_{\psi=0},
$$

where $v_{l}^{0}$ is the leading-order normal velocity generated by the leading-edge scattered outer solution, which can be determined from the acoustic pressure as

$$
v_{l}^{0}(\phi, 0)=\pi \mathrm{i} \int_{-\infty}^{\infty} \frac{\partial p_{l}^{0}}{\partial \psi}\left(\phi^{\prime}, 0\right) \mathrm{e}^{-\frac{\mathrm{i} k}{M}\left(\phi-\phi^{\prime}\right)} \operatorname{sgn}\left(\phi-\phi^{\prime}\right) d \phi^{\prime} .
$$

We now take the Laplace transform of (3.5.0.2) with respect to $\phi$, denoted by

$$
\tilde{G}(S, \xi)=\int_{0}^{\infty} G(\phi, \xi) e^{-S \phi} d \phi
$$

to find that

$$
\tilde{G}(S, \xi)=B(S) \mathrm{e}^{-\mathrm{e}^{-\pi \mathrm{i} / 4} \sqrt{2 S} \frac{M_{\infty}}{M} \xi},
$$


where $B(S)$ can now be obtained by evaluating the Laplace transform of $v_{l}^{0}$ given by (3.5.0.4). The Laplace transform can then be inverted to determine the transition solution.

The total far-field acoustic pressure emanating from the leading edge is given as a sum of the outer field determined in the previous section and the transition solution determined in this section. The transition solution does not appear directly in the far-field acoustics since it is clear from (3.5.0.6) that the transition solution decays exponentially in the transverse direction away from the aerofoil surface. It does, however, introduce a pressure discontinuity across the aerofoil, which must be corrected downstream of the trailing edge across the wake. This is done by the introduction of trailing-edge inner and transition solutions, where the inner solution matches onto an outgoing trailing-edge acoustic field, the analysis of which is detailed in the next section. The trailing-edge transition solution does not affect the far-field acoustics similarly to the leading-edge transition solution, therefore we only require explicit knowledge (which must be obtained numerically) of the leading-edge transition solution at the trailing edge, $(\phi, \psi)=\left(\phi_{e}, 0_{ \pm}\right)$, in order to complete our approximation for the far-field noise.

\subsection{Trailing-Edge Solutions}

Here we determine the solution in the trailing-edge inner region and the trailing-edge contribution to the outer region, denoted by (iv) and (ii) in Figure 3.1 respectively. The transition solution in the wake (region (v) in Figure 3.1) is not required for the acoustic far field, and is very similar to solutions found for background uniform flows in Chapters 1 and 2 , and therefore will not be discussed in much detail.

We shift coordinates to be aligned with the trailing edge, defining $\left(\phi_{t}, \psi_{t}\right)$ such that $(\phi, \psi)=\left(2+\phi_{t}+\epsilon \alpha_{t}, \psi_{t}\right)$. Here $\alpha_{t}=O(1)$ arises from the effect of thickness during the mapping of coordinates from physical space to $(\phi, \psi)$-space and $\alpha_{t}$ can be calculated in much the same way as was done in Chapter 1, (1.4.1.4), because in the inner region the aerofoil only sees a uniform flow. The transverse velocity of the incident gust solution at the trailing edge is still given by (3.2.0.7).

\subsubsection{Trailing-Edge Inner Solution}

We move to inner trailing-edge coordinates, $\left(\Phi_{t}, \Psi_{t}\right)=k\left(\phi_{t}, \psi_{t}\right)$. The trailing-edge inner acoustic solution, equivalent to (3.3.1.1), satisfies a dual integral equation equivalent to 
(3.3.1.2), which is

$$
\begin{array}{cc}
\int_{-\infty}^{\infty} \mathrm{e}^{\mathrm{i} \alpha \Phi_{t} / k} A(\alpha) P_{\text {out }}(\alpha, 0) d \alpha=-\Delta p\left(\Phi_{t}\right) / 2 & \Phi_{t}>0 \\
\int_{-\infty}^{\infty} \mathrm{e}^{\mathrm{i} \alpha \Phi_{t} / k} A(\alpha) V_{\text {out }}(\alpha, 0) d \alpha=-v_{g}\left(\Phi_{t}, 0\right) & \Phi_{t}<0 .
\end{array}
$$

Here all functions are written in terms of trailing-edge coordinates, $\alpha$ is redefined accordingly as the Fourier transform variable with respect to $\phi_{t}$, and $\Delta p\left(\Phi_{t}\right)$ is the inner approximation for the pressure jump across the trailing edge generated by the leadingedge solution. We separate the required inner solution, $p_{a}\left(\Phi_{t}, \Psi_{t}\right)$, into a term that corrects the pressure jump across the trailing edge, $p_{a, p}$, and a term that corrects for the zero normal velocity condition on the surface of the aerofoil, $p_{a, H}$. Using the notation from (3.3.1.1), we require

$$
\begin{aligned}
& \int_{-\infty}^{\infty} \mathrm{e}^{\mathrm{i} \alpha \Phi_{t} / k} A_{p}(\alpha) P_{\text {out }}(\alpha, 0) d \alpha=-\Delta p\left(\Phi_{t}\right) / 2 \quad \Phi_{t}>0, \\
& \int_{-\infty}^{\infty} \mathrm{e}^{\mathrm{i} \alpha \Phi_{t} / k} A_{p}(\alpha) V_{\text {out }}(\alpha, 0) d \alpha=0 \quad \Phi_{t}<0,
\end{aligned}
$$

and

$$
\begin{aligned}
\int_{-\infty}^{\infty} \mathrm{e}^{\mathrm{i} \alpha \Phi_{t} / k} A_{H}(\alpha) P_{\text {out }}(\alpha, 0) d \alpha=0 & \Phi_{t}>0, \\
\int_{-\infty}^{\infty} \mathrm{e}^{\mathrm{i} \alpha \Phi_{t} / k} A_{H}(\alpha) V_{\text {out }}(\alpha, 0) d \alpha=-v_{g}\left(\Phi_{t}, 0\right) & \Phi_{t}<0 .
\end{aligned}
$$

The solution of (3.6.1.2) and (3.6.1.3) is obtained using identical methods to those used at the leading edge in Section 3.3, and is presented in Appendix B. We use the solutions for $P_{\text {out }}^{0,1}$ as previously obtained in Section 3.3.2, but translated to the trailing-edge inner coordinate system. Taking the outer limit of the inner solutions, (B.1), and using the method of steepest descents yields

$$
\begin{aligned}
p_{a}^{t}\left(r_{t}, \theta_{t} \mid \eta_{t}\right) \sim & \left(\frac{\mathrm{i}}{2 \pi k r_{t}}\right)^{1 / 2} \frac{\kappa_{t}^{0}\left(k \beta_{t 0}\right)_{-} \sin \theta_{t}}{\left(1-M_{0}^{2} \sin ^{2} \theta_{t}\right)^{3 / 4}} \frac{\mathrm{e}^{\mathrm{i} k r_{t} \lambda_{t 0}\left(\theta_{t}\right)}}{1-\beta_{t 0} M\left(\eta_{t}\right)} \frac{C^{0}\left(k \beta_{t 0}\right) \tilde{\Omega}\left(\eta_{t}\right) \tilde{Q}^{0}\left(\eta_{t}\right) M\left(\eta_{t}\right)}{\kappa_{t}^{0}\left(k / M\left(\eta_{t}\right)\right)_{-} V_{\text {out }}^{0}\left(k \beta_{t 0}, 0\right)} \\
& {\left[1+\epsilon \sqrt{k}\left\{\frac{\tilde{Q}^{1}\left(\eta_{t}\right)}{\tilde{Q}^{0}\left(\eta_{t}\right)}+M^{\prime}\left(\eta_{t}\right)\left(\frac{P_{\text {out }}^{1}\left(k / M\left(\eta_{t}\right), 0\right)}{P_{\text {out }}^{0}\left(k / M\left(\eta_{t}\right), 0\right)}-\frac{V_{\text {out }}^{1}\left(k / M\left(\eta_{t}\right), 0\right)}{V_{\text {out }}^{0}\left(k / M\left(\eta_{t}\right), 0\right)}\right)\right.\right.} \\
& \left.\left.+\frac{d_{t}\left(k \beta_{t 0}, \Psi_{t}\right)-d_{t}\left(k \beta_{t 0}, 0\right)}{C^{0}\left(k \beta_{t 0}\right)}\right\}\right] \\
& +\left(\frac{\mathrm{i}}{2 \pi k r_{t}}\right)^{1 / 2} \frac{\kappa_{t}^{0}\left(k \beta_{t 0}\right)_{-} \sin \theta_{t} \mathrm{e}^{\mathrm{i} k r_{t} \lambda_{t 0}\left(\theta_{t}\right)}}{\left(1-M_{0}^{2} \sin ^{2} \theta_{t}\right)^{3 / 4}} \frac{G_{t, p}\left(k \beta_{t 0}\right) P_{\text {out }}^{0}\left(k \beta_{t 0}\right)}{V_{\text {out }}^{0}\left(k \beta_{t 0}\right)}
\end{aligned}
$$


as $k r_{t} \rightarrow \infty$, where $\lambda_{t 0}$ is the trailing-edge equivalent of $\lambda_{0}$, and $\beta_{t 0}$ is the trailing-edge equivalent of $\beta_{0}$ as defined in (3.3.2.9). The function $\kappa_{t}^{0}(\alpha)_{-}$is defined in Appendix $\mathrm{B}$ by

$$
\kappa_{t}^{0}(\alpha)_{+} \kappa_{t}^{0}(\alpha)_{-}=\frac{V_{\text {out }}^{0}(\alpha, 0)}{P_{\text {out }}^{0}(\alpha, 0)}
$$

hence using a trailing-edge form of $P_{\text {out }}$ given by (3.3.2.3), and using the leading-order relationship between $P$ and $V$ which can be obtained from (3.1.0.6), we find that

$$
\kappa_{t}^{0}(\alpha)_{-}=\frac{\sqrt{k-\alpha\left(1+M_{0}\right)}}{k-\alpha M_{0}} .
$$

The final term in (3.6.1.4) is in fact $O\left(k^{-1}\right)$ due to the scaling of the pressure jump term $G_{t, p}$, defined in (B.2c). The term involving $d_{t}\left(k \beta_{t 0}, \Psi_{t}\right)$ in (3.6.1.4) is negligible to the orders retained here since in the trailing-edge region the terms $\sigma_{i}$ in (3.1.0.6) are negligible (because there is less curvature of the streamlines at the trailing edge than at the leading edge). The choice of $C^{0}$ is again given by (3.3.2.4), which now ensures that the trailing-edge inner solution has a $\sin \theta / 2$ directivity pattern. This is the same directivity pattern found for sound- and gust-aerofoil interaction in steady uniform flow in Chapters 1 and 2 respectively. Once again we know that the shear flow directivity pattern should match the uniform flow directivity pattern to leading order, since in the trailing-edge inner region the aerofoil only experiences the local Mach number $M_{0}$.

The terms in square brackets in (3.6.1.4) represent the scattering of the acoustic pressure associated purely with the gust in the shear flow (in uniform flow a gust is pressure-free, and these terms vanish). Whilst the contribution of these terms appears to be the same order as the contribution of the leading-edge terms, (3.3.2.5), we in fact find that it is at least $O(M)$ smaller due to $\kappa_{t}^{0}\left(k / M\left(\eta_{t}\right)\right)_{-}$having a singularity at $\eta_{t}=0$. We mentioned at the end of Section 3.3.1 that to evaluate the pressure $p_{a}(r, \theta)$ given as an integral over $\eta$ of $p_{a}(r, \theta \mid \eta)$ in (3.3.1.3), we consider only sharply peaked vorticity distributions where $\eta=0$ dominates. At $\eta_{t}=0, \kappa_{t}^{0}\left(k / M\left(\eta_{t}\right)\right)_{-}=0$ therefore before applying Laplace's method we must take an expansion of $\kappa_{t}^{0}\left(k / M\left(\eta_{t}\right)\right)_{-}$as $\eta_{t} \rightarrow 0$. This expansion reduces the apparent order of the first term in (3.6.1.4) by at least $O(M)$ (the true scaling depends on how the vorticity distribution relates to $k$ ), thus the true order of these first terms should be treated as $O(M)$ smaller than the leading-edge contribution to the far-field acoustics. The final term in (3.6.1.4) accounts for the rescattering of the leading-edge acoustic field by the trailing edge, and, as expected by comparison with the uniform flow case, is $O\left(k^{-1 / 2}\right)$ smaller than the leading-edge solution. We write the 
outer limit of the trailing-edge inner solution (once integrated by $\eta_{t}$ ) as

$$
\frac{1}{\sqrt{k r_{t}}}\left(M \mathcal{D}_{t_{1}}+\frac{1}{\sqrt{k}} \mathcal{D}_{t_{2}}\right) \mathrm{e}^{\mathrm{i} k r_{t} \lambda_{t 0}\left(\theta_{t}\right)}
$$

where $\mathcal{D}_{t_{2}}$ is formally the same order as $\mathcal{D}_{l}$, but $\mathcal{D}_{t_{1}}$ could be smaller (depending on the choice of vorticity distribution). We set $\mathcal{D}_{t}=M \mathcal{D}_{t_{1}}+k^{-1 / 2} \mathcal{D}_{t_{2}}$.

\subsubsection{Trailing-Edge Outer Solution}

The trailing-edge outer solution is found in a similar way to the leading-edge outer scattered solution, assuming a form

$$
p_{t}=A_{t}\left(r_{t}, \theta_{t}\right) \mathrm{e}^{\mathrm{i} k \varrho_{t}^{0}\left(r_{t}, \theta_{t}\right)+\mathrm{i} k \epsilon \varrho_{t}^{1}\left(r_{t}, \theta_{t}\right)+O(\epsilon)}
$$

We find that

$$
A_{t}\left(r_{t}, \theta_{t}\right)=D_{t}(\theta)\left(k r_{t}\right)^{-1 / 2}, \quad \varrho_{t}^{0}\left(r_{t}, \theta_{t}\right)=r \lambda_{t \infty}\left(\theta_{t}\right)
$$

where $\lambda_{t \infty}\left(\theta_{t}\right)$ is the corresponding trailing-edge function to $\lambda_{\infty}(\theta)$, and $\varrho_{t}^{1}$ is given by the corresponding trailing-edge formulation of (3.4.0.10). We match this to the trailingedge inner solution by setting $D_{t}\left(\theta_{t}\right)$ equal to $\mathcal{D}_{t}\left(\theta_{t}\right) s_{t}\left(r_{t}, \theta_{t}\right)$, where the first factor arises from the directivity emerging from the inner region in (3.6.1.4) and the second factor accounts for the variation in ray tube area as the sound propagates though the shear see equation (3.4.0.3). The total far-field acoustic pressure emanating from the trailing edge then takes the form

$$
\frac{D_{t}\left(\theta_{t}\right)}{\sqrt{k r_{t}}} \exp \left(\mathrm{i} k r_{t} \lambda_{t \infty}\left(\theta_{t}\right)+\frac{1}{2} \mathrm{i} k \epsilon \int_{0}^{\phi_{t}+\psi_{t}} L_{t}\left(\chi, \psi_{t}\right) d \chi\right) .
$$

Again, this is only valid in the far field, where the Mach number approaches $M_{\infty}$.

\subsubsection{Trailing-Edge Transition Solution}

We require a trailing-edge transition solution to ensure continuous pressure across the entire wake; the inner trailing-edge solution only cancelled the pressure jump at the trailing-edge, and not along the entire wake. We solve similarly to the leading-edge transition solution, supposing the solution takes the form

$$
p_{t t r}=\frac{1}{\sqrt{k}} G_{t}\left(\phi_{t}, \xi_{t}\right) \mathrm{e}^{\mathrm{i} k \varrho_{t}^{0}\left(\phi_{t}, 0\right)}
$$


where $\xi_{t}=\sqrt{k} \frac{M_{\infty}}{M} \psi_{t}$ and $G_{t}$ satisfies

$$
\frac{\partial^{2} G_{t}}{\partial \xi_{t}^{2}}+2 \mathrm{i} \frac{\partial G_{t, t}}{\partial \phi_{t}}=0
$$

We factor the Mach number dependence into the transition variable, $\xi_{t}$ so that our trailing-edge transition problem resembles previous trailing-edge problems in Chapters 1 and 2. We require the pressure jump across the wake to be cancelled by $G_{t}$ and we insist on having continuous normal velocity across the wake, hence we need $G_{t}$ to be anti-symmetric in $\xi_{t}$ near $\xi_{t} \rightarrow 0$, and

$$
G_{t}\left(\phi_{t}>0,0_{+}\right)=-\Delta p\left(\phi_{t}\right) / 2
$$

We also apply the radiation condition for large $\xi_{t}$ (i.e. $G_{t} \rightarrow 0$ as $\xi_{t} \rightarrow \infty$ ), hence by using a Laplace transform we find that

$$
G_{t}\left(\phi_{t}, \xi_{t}\right)=-\frac{\mathrm{e}^{-\mathrm{i} \pi / 4}}{\sqrt{8 \pi}} \int_{0}^{\phi_{t}} \frac{\xi_{t} \Delta p(\chi)}{\left(\phi_{t}-\chi\right)^{3 / 2}} \mathrm{e}^{\mathrm{i} \xi_{t}^{2} / 2\left(\phi_{t}-\chi\right)} d \chi
$$

This solution is of the same form as the trailing-edge transition solution given in Chapter 1 by (1.4.3.1b), and the matching with the inner and outer solutions follows in a similar manner. The transition solution matches to the inner solution, which can be seen by considering the small $\phi_{t}, \xi_{t}$ behaviour. The trailing-edge outer solution already contains a term that aids the cancellation of the pressure discontinuity (a $\Delta p\left(\Phi_{t}\right)$ term which is equal to $\Delta p(0))$. Thus the outer limit of the transition solution and the transition limit of the outer solution have a common term involving this pressure jump, similar to the common term found in the uniform flow analysis in Chapter 1 . The existence of the common term ensures matching, but we must take care to subtract the common term from the final trailing-edge solution.

\subsection{Total far-field solution}

The total far-field solution is obtained by summing the leading-edge outer solution, from (3.3.2.5) and (3.3.2.17) integrated in (3.3.1.3), and the trailing-edge outer solution, from (3.6.1.4) integrated in (3.3.1.3). Both the leading- and trailing-edge transition solutions, and the common term for the trailing-edge solutions are negligible in the far field at angles where $M \approx M_{\infty}$ (i.e. everywhere except at small angles close to the downstream direction, $\theta=0$ ). We have previously neglected these small angles because during our 
outer solution analysis we supposed $M \approx M_{\infty}$ in the far field. Therefore our total result is obtained solely from the leading- and trailing-edge outer solutions, with no contributions from the transition solutions, and it is valid only where $M \approx M_{\infty}$ in the far field.

In the far field, the coordinate transformation between leading-edge and trailing-edge polar coordinates is given by

$$
r_{t} \approx r-\left(2+\alpha_{t} \epsilon\right) \cos \theta, \quad \theta_{t} \approx \theta-\pi+\pi \operatorname{sgn}(\psi)
$$

which allows the final solution to be written in terms of leading-edge variables $(r, \theta)$. The far-field acoustic pressure can then be written as

$$
\frac{1}{\sqrt{k r}}\left(D_{l}(\theta)+D_{t}(\theta) \mathrm{e}^{\mathrm{i} k \varrho_{s}(r, \theta)}\right) \mathrm{e}^{\mathrm{i} k r \lambda_{\infty}(\theta)+\frac{1}{2} \mathrm{i} k \epsilon \int_{0}^{\phi+\psi} L(\chi, \psi) d \chi},
$$

where $D_{l, t}$ are defined in (3.4.0.11) and (3.6.2.3). In the far field, the leading- and trailing-edge ray fields interact with a phase shift

$$
k \varrho_{s}(r, \theta)=k\left(\varrho_{t}^{0}\left(r_{t}, \theta_{t}\right)+\epsilon \varrho_{t}^{1}\left(r_{t}, \theta_{t}\right)-\varrho^{0}(r, \theta)-\epsilon \varrho^{1}(r, \theta)\right) .
$$

The leading-order contribution to the phase shift, $k\left(\varrho_{t}^{0}-\varrho^{0}\right)$, is given by

$$
-2 k \lambda_{\infty}(\theta) \cos \theta
$$

The $O(\epsilon k)$ phase shift term given by $\epsilon k\left(\varrho_{t}^{1}-\varrho^{1}\right)$, is approximated by

$$
\frac{\epsilon k}{2} \int_{0}^{2} L(\chi, \psi) d \chi
$$

in the far field.

\subsection{Results}

In this section we present results for the far-field pressure directivity generated by gustaerofoil interaction in steady shear flow. The analysis presented so far is applicable to a general thin uncambered aerofoil, and, subject to the restrictions described in Section 3.2 , to a general mean shear distribution. For definiteness, we consider a gust interacting with a symmetric Joukowski aerofoil of thickness $\epsilon \ll 1$ and chord length 2 in a steady 
Gaussian shear flow defined by

$$
U_{0}(y)=\left(\mathcal{U}_{0}-1\right) e^{-y^{2}}+1
$$

\subsubsection{Evaluation of the Streamfunction}

The streamfunction for the parallel shear, $\psi^{0}(y)$, is simply

$$
\psi^{0}(y)=\left(\mathcal{U}_{0}-1\right) \frac{\sqrt{\pi}}{2} \operatorname{erf}(y)+y
$$

Let $\epsilon \psi^{1}(x, y)$ be the perturbation to the streamfunction caused by the presence of the thin Joukowski aerofoil. We first note that in our limit of low Mach number flow, the effects of compressibility on $\epsilon \psi^{1}(x, y)$ do not arise to the order considered, and we can therefore use the work of Sowyrda (1958), who considered incompressible steady flow around a thin Joukowski aerofoil. Secondly, we recall that we need only find an approximation to $\psi^{1}$ close to the aerofoil because, as mentioned at the end of the previous section, the $O(\epsilon \sqrt{k})$ far-field acoustic terms are dominated by the inner effects at the leading and trailing edges. We know that far from the aerofoil, $\psi^{1} \rightarrow 0$, thus here we only focus on finding $\psi^{1}$ close to the aerofoil.

To determine the perturbation to the streamfunction caused by the aerofoil, we consider the shear flow, (3.8.0.1), acting on an infinitely long circular cylinder of radius $1+\epsilon$ which may be thought of as purely two-dimensional. We map this conformally to the Joukowski aerofoil, with leading edge at $(x, y)=\left(-4 \epsilon^{2}, 0\right) \approx(0,0)$ and trailing edge at $(x, y)=(2,0)$ via the Joukowski transformation

$$
2 z=\zeta-\epsilon+\frac{1}{\zeta-\epsilon}+2
$$

Here $z=x+i y$ are the coordinates for the aerofoil and $\zeta=r e^{i \theta}$ are the coordinates for the circle.

The Joukowski transformation to $O(\epsilon)$ is

$$
z \approx \zeta+\frac{1}{\zeta}+2-\epsilon+\frac{\epsilon}{\zeta^{2}} .
$$

The individual coordinate transformation from $(r, \theta)$ to $(x, y)$ is

$$
x \approx\left(r+\frac{1}{r}\right) \cos \theta+2+\epsilon\left(\frac{\cos 2 \theta}{r^{2}}-1\right),
$$




$$
y \approx\left(r-\frac{1}{r}\right) \sin \theta-\frac{\epsilon}{r^{2}} \sin 2 \theta
$$

Since we assume at first order the flow is incompressible, we require $\psi^{1}$ to satisfy the equations given in Sowyrda (1958);

$$
\begin{array}{rlrl}
\nabla^{2} \psi^{1} & =0, \\
\frac{\partial \psi^{1}}{\partial n} & =-\boldsymbol{U}_{\mathbf{0}} \cdot \boldsymbol{n} & & \text { on the surface of the aerofoil, } \\
{\left[\frac{\partial \psi^{1}}{\partial r}\right]} & =0 & & \text { on the trailing edge, } \\
\psi^{1}(r, \theta) & \rightarrow 0 & \text { as } r \rightarrow \infty .
\end{array}
$$

These have imposed a zero velocity boundary condition on the aerofoil surface, the Kutta condition across the trailing edge, and a radiation condition at infinity. We can write the solution as

$$
\psi^{1}(r, \theta)=b_{0} \theta+\sum_{n=1}^{\infty} \frac{a_{n}}{r^{n}} \cos n \theta+\frac{b_{n}}{r^{n}} \sin n \theta,
$$

so we now require

$$
\left.\frac{\partial \psi^{1}}{\partial \theta}\right|_{r=1+\epsilon}=-2 \mathcal{U}_{0}(\cos \theta-\cos 2 \theta) .
$$

To orders retained in our calculations, we therefore set

$$
b_{0}=0, \quad b_{1}=-2 \mathcal{U}_{0}, \quad b_{2}=\mathcal{U}_{0}, \quad a_{i}=b_{i}=0 \quad \text { otherwise }
$$

Hence

$$
\psi(x, y) \approx \psi^{0}(y)+\epsilon \mathcal{U}_{0}\left(\frac{1}{r^{2}} \sin 2 \theta-\frac{2}{r} \sin \theta\right)
$$

close to the leading and trailing edges of the Joukowski aerofoil. Having calculated $\psi$ to two orders of magnitude, we can calculate the $N_{i}$ required to obtain the acoustic far field using the expressions in Section 3.3.2.

\subsubsection{Far-Field Results}

We define the far field as being a distance, $r$, far enough away from the aerofoil so that the mean flow is approximately uniform, and amplitude terms of $O(1 / r)$ are negligible compared to terms retained in the asymptotic solution. Since there is no Rayleigh distance for this gust-aerofoil problem, we choose $r=25$ in all following results to illustrate the far-field behaviour. 
In Figure 3.2 we consider the effect of altering the strength of the mean shear, characterised by the parameter $S=\left(M_{\infty}-M_{0}\right) / M_{\infty}$, on the leading-edge directivity. Here we set $\epsilon=0$, so that the aerofoil reduces to a flat plate, and plot the quantity $\left|D_{l}(\theta)\right|$ as defined in equation (3.4.0.11). When $S=0$ the directivity pattern takes the familiar form $\cos \theta / 2$, which is characteristic of low Mach number uniform flow gust-aerofoil interaction. Varying $S$ away from zero has a significant effect; when the shear is jetlike $(S>0)$ the directivity is particularly reduced in the downstream direction, with little effect upstream, whereas for wake-like shear $(S<0)$ the directivity is reduced predominantly upstream. Mathematically, these directivity effects may be coming from two places; first, in (3.3.2.5) through the terms dependent on $\beta_{0}$; and second, from the ray-tube area scaling factor $s(r, \theta)$ in (3.4.0.2). We have investigated the relative effects of both sets of terms, and have found that the directivity variations seen in Figure 3.2 are arising primarily from the second effect of the shear increasing the ray tube area (and therefore decreasing the pressure amplitude along the ray) in the downstream/upstream directions for $S$ positive and negative respectively. See Figure 3.3.

In Figure 3.4 the effects of aerofoil thickness on the leading-edge directivity are considered. In significant shear, $S=0.333$, we see that increasing the thickness from zero changes the nature of the directivity. The leading-edge sound is made up of two contributions; the leading-order term corresponding to flat-plate scattering, see (3.3.2.5), and an additive correction term of relative size $O(\epsilon \sqrt{k})$ to account for the effects of thickness in the leading-edge region, see (3.3.2.17). The interference between these two sources in shear gives rise to the lobular directivity pattern seen in Figure 3.4. Note that the contribution from (3.3.2.17) takes the same form as the contribution from (3.3.2.5), but with a multiplicative correction factor which involves several effects (see the brief discussion following (3.3.2.17)). However, the variation with observer angle, $\theta$, seen in Figure 3.4 can only arise from the term in this correction factor involving $d\left(k \beta_{0}, k \psi\right)$, which in turn arises from the term on the right hand side of equation (3.1.0.9a), i.e. from the interaction between the leading-order scattered field and the non-uniformity of the mean shear flow. We stress that the interference within the leading-edge field is only present in shear flow.

We must now choose an upstream vorticity distribution in order to obtain quantitative results for the trailing-edge noise. We therefore choose $\tilde{\Omega}(\eta) \sim \mathrm{e}^{-k \eta^{2}}$. In Figure 3.5 we see the relative effects of the two interactions generating sound at the trailing edge of the aerofoil. The sound generated by the rescattering of the leading-edge field by the trailing edge, given by $k^{-1 / 2} \mathcal{D}_{t_{2}}$ in (3.6.1.7), is familiar as it is the sole noise from the trailing edge in uniform flow gust-aerofoil interaction. The scattering of the pressure associated 


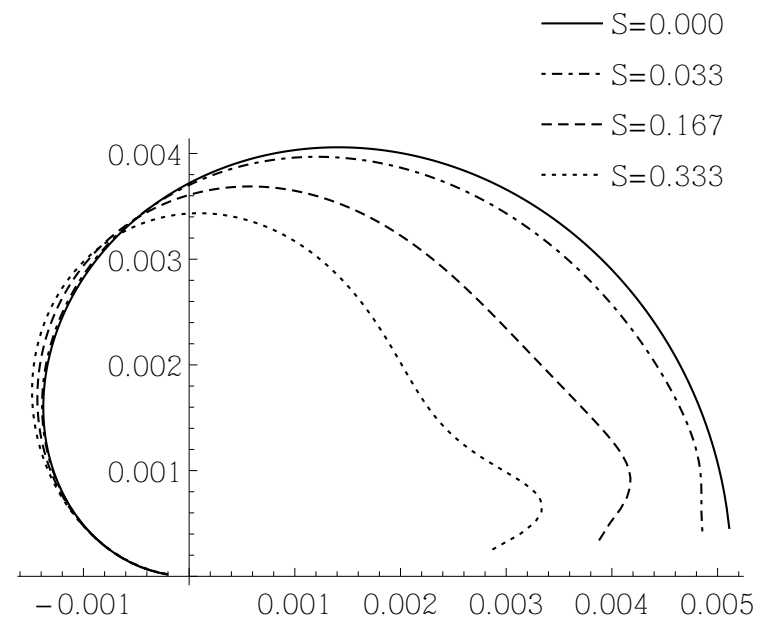

(a) Positive $S$, with $M_{\infty}=0.3$.

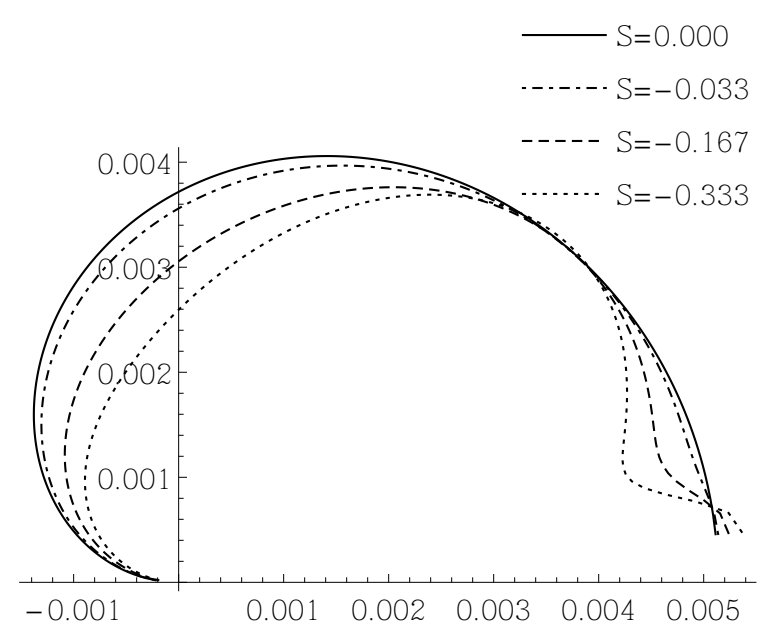

(b) Negative $S$, with $M_{0}=0.3$.

Figure 3.2: Leading-edge far-field acoustic pressure directivity for a flat plate, with $k=10$ and varying strengths of shear, $S=\left(M_{\infty}-M_{0}\right) / M_{\infty}$.

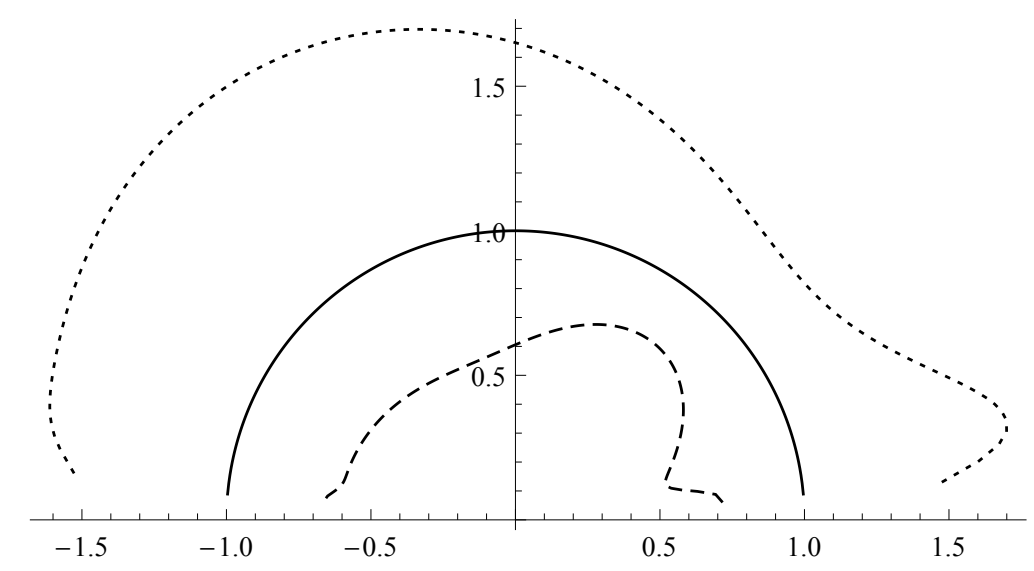

Figure 3.3: Magnitude of the scaling factor, $s$, for various shear flow profiles; dotted $M_{0}=0.3, M_{\infty}=0.2$, solid $-M_{0}=M_{\infty}=0.3$, dashed $-M_{0}=0.2, M_{\infty}=0.3$, at a distance $r=25$. 


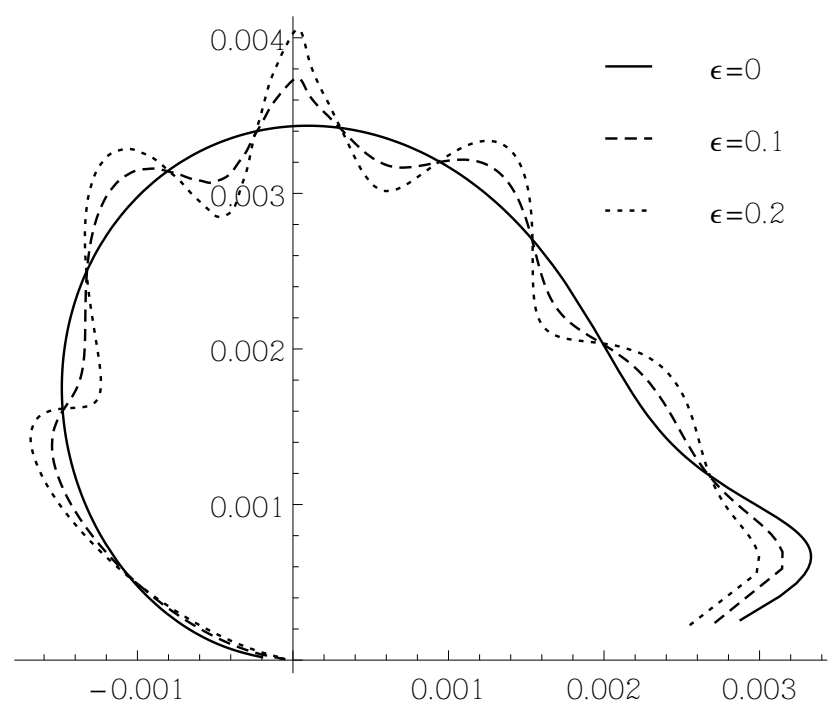

Figure 3.4: Leading-edge far-field acoustic pressure directivity with $k=10, M_{0}=0.2$, $M_{\infty}=0.3$, and $S=0.33$, for Joukowski aerofoils of varying thickness, denoted by $\epsilon$.

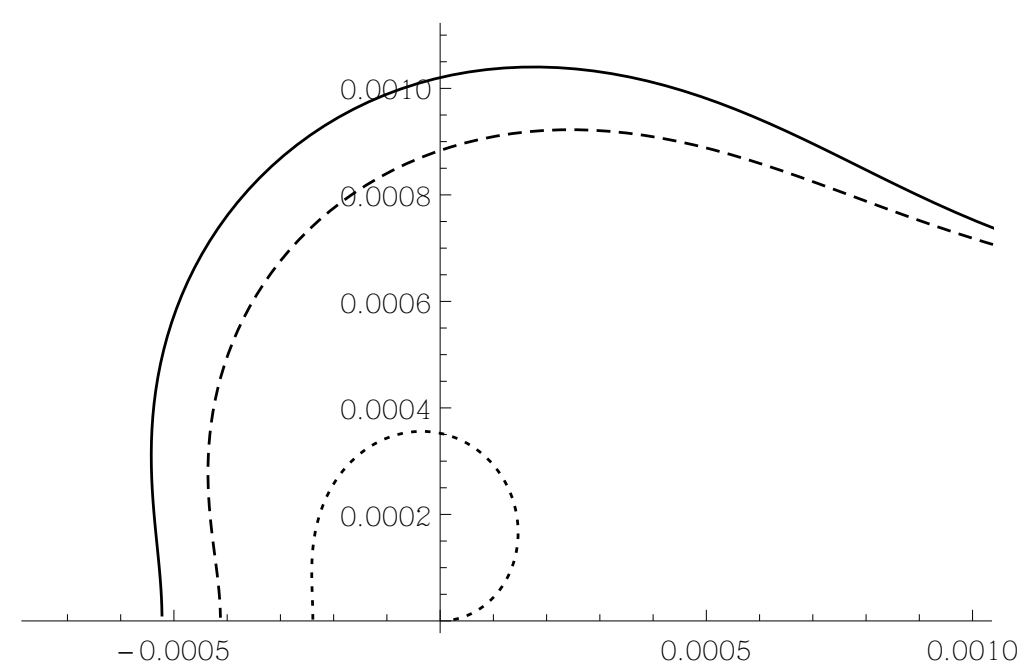

Figure 3.5: Trailing-edge far-field pressure directivity with $S=0.333, k=10, \epsilon=0.1$. The solid line denotes the total trailing-edge directivity. The dashed line denotes the contribution from the rescattering of the leading-edge field by the trailing edge, and the dotted line denotes scattering by the trailing-edge of pressure associated with the gust. 
with the gust, $M \mathcal{D}_{t_{1}}$ from (3.6.1.7), is peculiar to shear flow interactions, and as we see in Figure 3.5 has a non-negligible effect on the total trailing-edge contribution upstream of the aerofoil. For our chosen vorticity distribution, $M \mathcal{D}_{t_{1}}$ is formally $O\left(k^{-1 / 4}\right)$ smaller than $k^{-1 / 2} \mathcal{D}_{t_{2}}$, which is most noticeable in the upstream region of Figure 3.5. Downstream of the aerofoil, the size difference is increased, since this scattering of pressure (the dotted line) takes a $\sin \theta / 2$ type directivity pattern. At the trailing edge, as with the leading edge, there are two contributions to the acoustic solution; one from the rescattering of the leading-edge field, and one from the interaction of the unsteady self-noise with the perturbed steady flow. However, at the trailing edge, the perturbation to the steady mean flow is smaller than at the leading edge by a factor of $k^{-1 / 2}$, hence the interaction of these two sources should be weaker than the interaction of the two leading-edge sources. Furthermore, by considering Figure 3.5, we see that the contribution to the trailing-edge field due to the scattering of gust self-noise is much smaller than the contribution due to the rescattering of the leading-edge field. We therefore do not observe a modulated trailing-edge field despite (as with the leading-edge field) there being multiple types of interaction occurring.

We now consider the total scattered acoustic pressure as the sum of leading- and trailing-edge fields. In Figure 3.6 we consider the far-field pressure directivity in the two very low shear cases $S= \pm 0.03$ for the flat plate, $\epsilon=0$. The significant modulation of the directivity is now caused by the interference between the leading- and trailing-edge fields, and is of course absent in the comparable plots of just the leading-edge flat-plate field (see Figure 3.2). We repeat these flat-plate calculations in Figure 3.7, but now with significant shear, and similar directivity patterns are again observed. Note that positive shear significantly increases/decreases the sound level in the upstream/downstream directions respectively, and vice versa for negative shear. This effect cannot be explained by simple ray tracing arguments, which would suggest that rays in positive/negative shear flow would tend to bend in the direction of decreasing/increasing $\theta$ (see Amiet (1978)). Rather, the refraction experienced by the leading- and trailing-edge fields is the same as they propagate through the shear, therefore the pattern seen in Figure 3.7 is influenced by the interaction of the two fields via their phase shift, $\varrho_{s}$, and their amplitudes (which both contain the scaling term, $s$, yielding the patterns shown in Figure 3.3). The refraction experienced by rays from the leading and trailing edges is contained within the overall phase function multiplying the total far field solution, (3.7.0.2), thus a directivity pattern does not pick out these effects.

In Figure 3.8 we introduce non-zero thickness to the finite-chord total far-field pressure results. We observe that increasing the thickness predominantly alters the upstream 


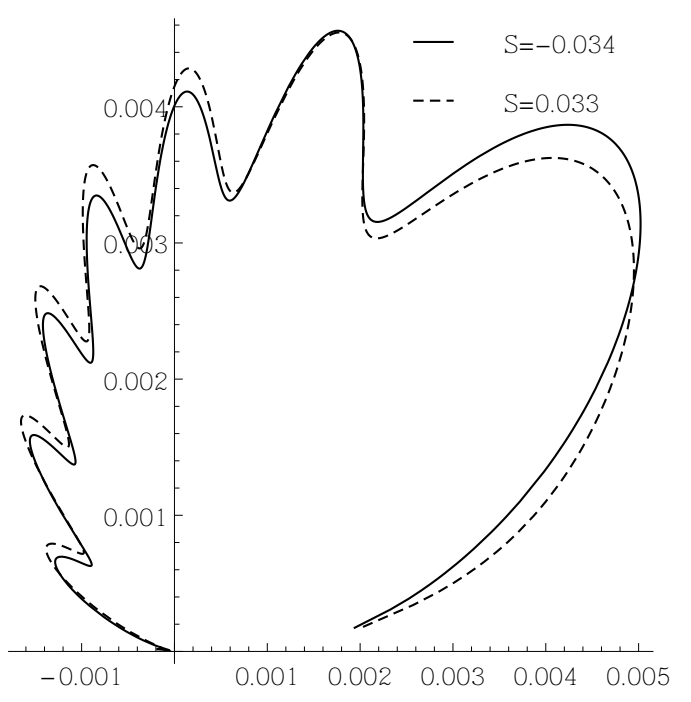

Figure 3.6: Far-field pressure directivity at $r=25$ in almost uniform Gaussian shear flow with $M \approx 0.3, k=10$, and $\epsilon=0$.

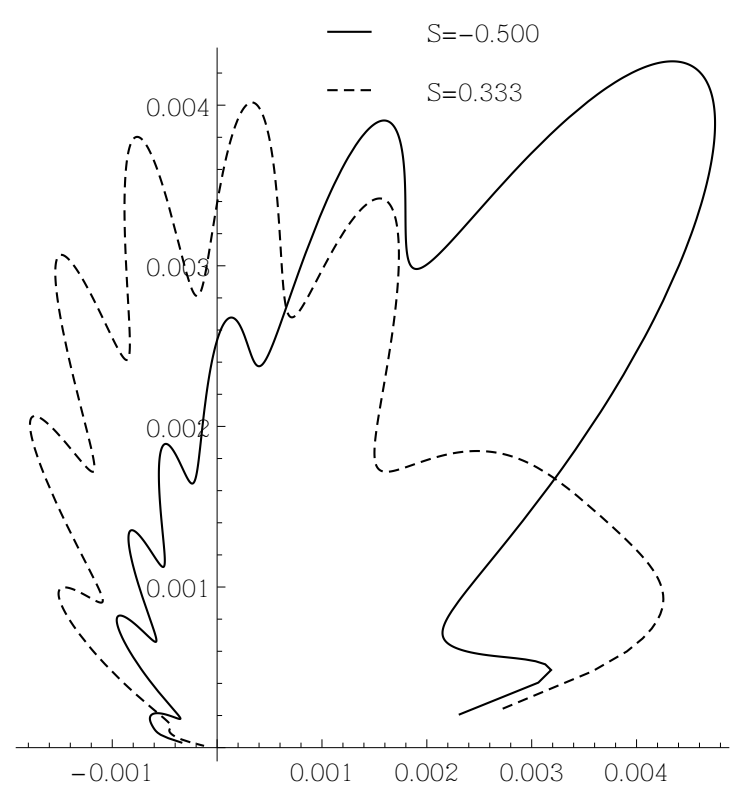

Figure 3.7: Far-field pressure directivity at $r=25$ for jet-like and wake-like shear flows, with $k=10$, and $\epsilon=0$.

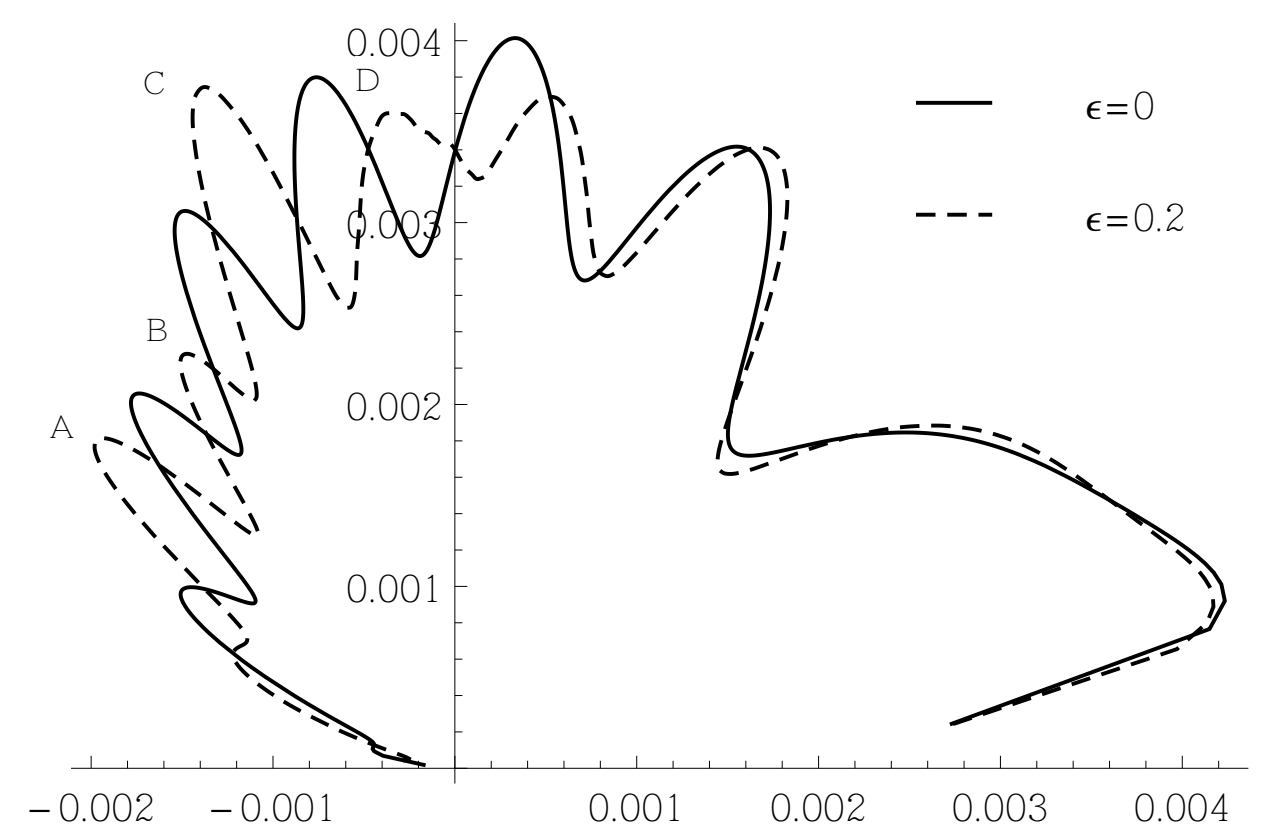

Figure 3.8: Far-field pressure directivity at $r=25$ with $M_{0}=0.2, M_{\infty}=0.3, k=10$, and varying thickness. 


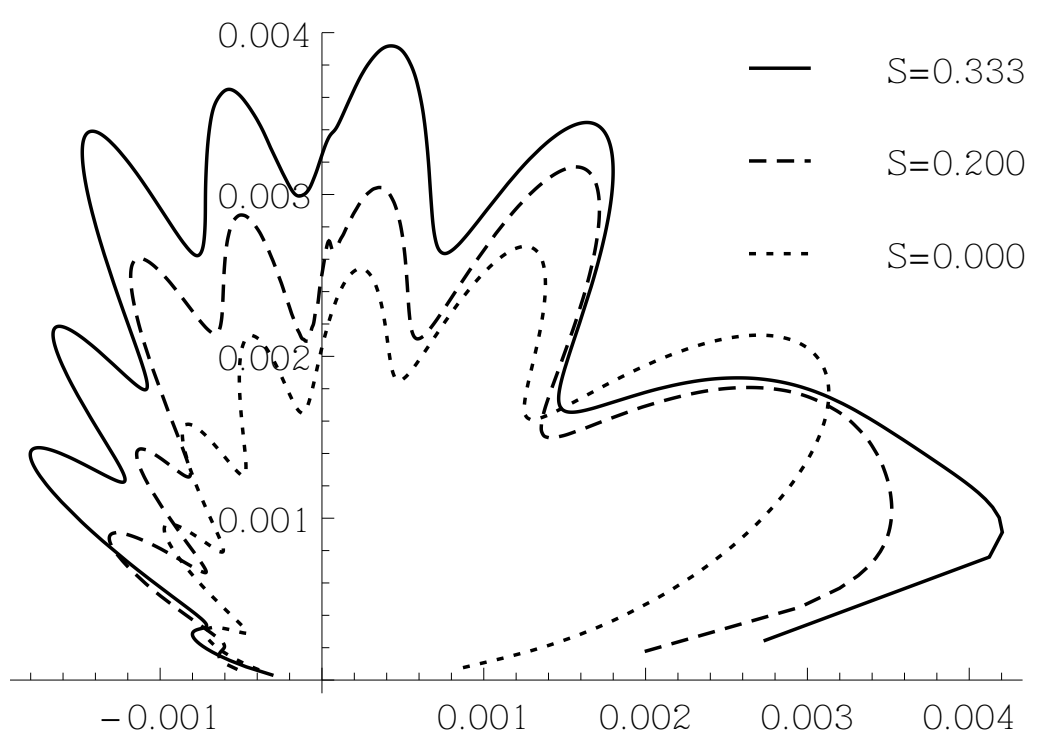

Figure 3.9: Far-field pressure directivity at $r=25$ with $M_{0}=0.2, k=10, \epsilon=0.1$ and varying $S$.

far-field pressure directivity, and one of these effects is to make the lobes more uneven. For non-zero thickness we see that the lobes labelled by A, B, C, and D have markedly different amplitudes (notably the lobes $\mathrm{A}$ and $\mathrm{C}$ are very large but $\mathrm{B}$ and $\mathrm{D}$ are less pronounced). For zero thickness, each lobe has a roughly equal amplitude in the upstream region. This uneven lobe pattern arises because of the leading-edge interaction seen in Figure 3.4 being superimposed on the interaction between the leading- and trailing-edge ray fields. Even-sized lobes are produced by leading-trailing interaction, as seen in Figure 3.7, but on introducing leading-edge interaction by allowing for non-zero thickness, the overall pattern of the total directivity field becomes modulated by an oscillating leadingedge field, thus allowing the uneven lobes to appear. This characteristic of the far-field pressure directivity for a finite length aerofoil of non-zero thickness is peculiar to shear flow interactions, as it is the interaction of the two leading-edge sources that creates it. Another upstream effect we see in Figure 3.8 due to variations in thickness is the positioning of the lobes and overall magnitude of the pressure. We see little difference in the field downstream of the aerofoil. We expect to see the most difference to the far-field pressure in the upstream region as we vary thickness because it is the blocking of the horizontal gust velocities, and scattering of gust self-noise by the nose of the aerofoil that is a new important source of noise for aerofoils of non-zero thickness.

In Figure 3.9 we show the effects of altering the shear strength, $S$, whilst maintaining a fixed value of $M_{0}$. It is important we mention that for this figure, $M_{0}$ is fixed, because 


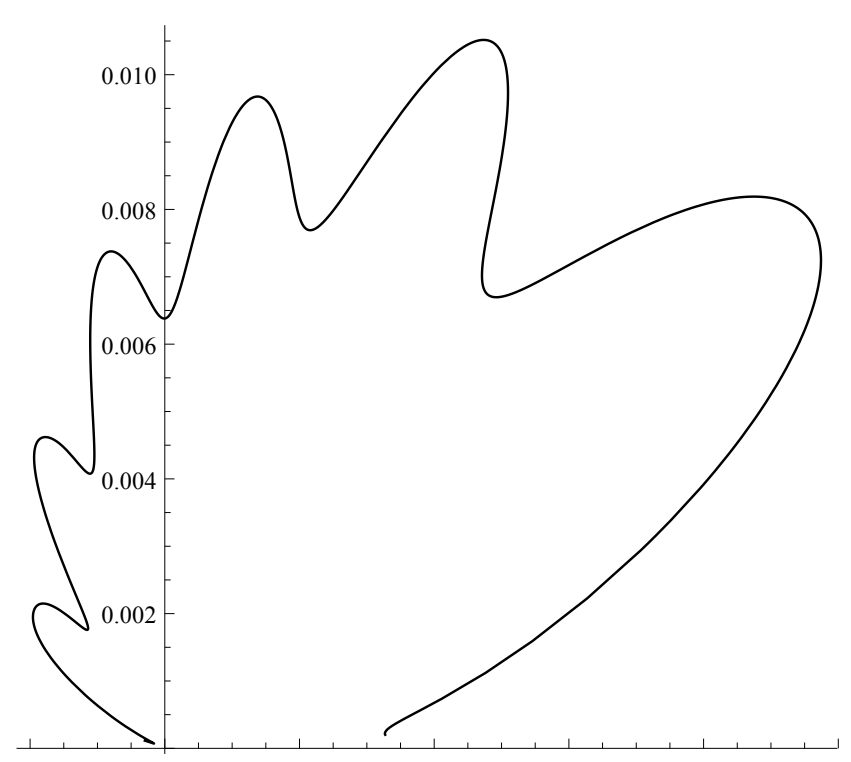

Figure 3.10: Far-field pressure directivity for gust-aerofoil interaction in uniform flow with $M=0.6$ around a Joukowski aerofoil of thickness 0.1 , and frequency (as defined in Chapter 2) 10.

we then know to attribute any variation in lobe position to the variation in $M_{\infty}$. This is sensible, as it is $M_{\infty}$ that has a dominant effect in the phase shift between the leadingand trailing-edge ray fields which therefore governs the leading-trailing interaction in the far field hence the positioning of the lobes. We see that as the strength of the shear is decreased towards zero, the overall shape of the directivity pattern reduces to a familiar pattern seen in uniform flow for purely transverse gusts, as illustrated by Figures 3.10, and 2.12 from Chapter 2. As $S$ increases away from 0 , the overall shape of the directivity pattern distorts in accordance to the ray tube scaling term, $s$, as seen in Figure 3.3. Once again, for significant shear and non-zero thickness, we see uneven lobes in Figure 3.9 due to the leading-edge interaction combining with the leading-trailing interaction.

\subsection{Conclusions}

We have constructed a model for the sound generated by gust-aerofoil interaction in background parallel shear flow, using asymptotic analysis in the limits of high gust frequency, and small but non-zero aerofoil thickness and Mach number. We have determined the first two terms in both the amplitude and the phase of the scattered pressure in the far field.

In uniform mean flow, as studied by Myers \& Kerschen (1997), Tsai (1992) and in Chapter 2 , the sound is dominated by the interaction of the gust with the leading edge 
of the aerofoil and the scattering of the leading-edge acoustic field by the trailing edge, with the latter being formally $O\left(k^{-1 / 2}\right)$ smaller than the former. We have shown that this feature is also present in shear flow, but that in addition the trailing edge itself acts as a noise source, as it scatters the hydrodynamic pressure associated with the gust into outgoing acoustic waves propagating to infinity. This trailing-edge noise is formally the same order in $k$ as the leading-edge noise, however in practice we found it to be $O(M)$ smaller (which is non-negligible) due to the restriction on the flow. These imposed flow conditions, of a sharply peaked vorticity distribution, and a parallel shear flow, permit gust-induced pressure fluctuations in the flow field which convect with the mean flow and are dominated in line with the leading edge. The leading edge interacts with the dominant self-noise component, blocking it from interacting with the trailing edge (or allowing the trailing edge only to interact with the scattered component from the leading edge). Therefore all gust-induced pressure fluctuations reaching the trailing edge are smaller than those approaching the leading edge, and in the case presented in this section, were found to be $O(M)$ smaller.

We have seen that changing the shear flow can have a strong effect on the directivity. The clearest effect is seen in Figure 3.2 where we found that wake-like, $S>0$, and jetlike, $S<0$ shear flows produce significantly different leading-edge directivity patterns, governed predominantly by the ray tube scaling factor, $s$, seen in Figure 3.3. This is also evident in Figure 3.7, where we plot the far-field pressure for a zero-thickness aerofoil for jet-like and wake-like shears, however we see an additional feature caused by introducing the trailing-edge field; the lobed directivity pattern arises from the phase shift associated with the differing paths from the leading and trailing edges to the observer, and by changing the shear one can make significant changes to this phase shift and hence to the interference pattern in the far field. We have calculated the first two terms (specifically $O(k)$ and $O(1))$ in the phase of the leading- and trailing-edge components, and therefore effectively the first two terms in the phase shift between them. Changing the background shear flow changes the leading-order phase term, and therefore has a more significant effect than, for instance, changing the aerofoil geometry, which only affects the secondorder phase term.

In addition, we have identified another mechanism by which the shear flow modifies the directivity, this time associated with the aerofoil thickness. This involves two stages. First, the leading-order field from the leading edge (which is caused by the momentumblocking of the incident gust by the aerofoil surface) and the second-order field from the leading edge (which is $O(\epsilon \sqrt{k})$ smaller than the leading-order term and is caused by the interaction between the unsteady flow and the non-uniform mean flow close to the thick 
leading edge) interfere with each other - see Figure 3.4. Second, this total leading-edge field interferes with the trailing-edge field to produce a modulated far-field directivity, see Figure 3.9. This creates a triple interaction which is not present in zero shear, because in the zero shear case the two components of the leading-edge field are in phase with each other, and the first stage of the interference does not occur. The triple interaction is also not present for zero thickness because the second-order leading-edge term is clearly zero in this case.

Our theory holds for more complicated parallel shear flows than the symmetric, single maximum/minimum case presented here, but in that case more involved numerical calculations would have to be included in order to evaluate the far-field scattered sound pressure. Similarly, it would also be possibly to consider asymmetric aerofoil cases by including the effects of angle of attack and camber on the mean flow, the gust evolution and the sound generation, but again significant additional complexity would be introduced. The issue of extending our work to $O(1)$ subsonic Mach numbers, however, seems much more difficult, not least because the small Mach number limit has allowed us to complete asymptotic calculations which otherwise appeared intractable at various points. Even so, we believe that the physical insights we have derived have broad application in a range of areas. We are not aware of any fully-computational approaches to this problem, but given the experience of gust-aerofoil interaction in uniform flow we believe that our approach would provide a useful complement to fully numerical computations in the high-frequency regime. 


\section{List of Symbols for Chapter 3}

$A \quad$ function found in the Wiener-Hopf solution to the inner problems.

$\mathcal{D}$ directivity of the outer limit of the leading-edge inner solution. Total leading- and trailing-edge directivities in the far field are $D_{l, t}$ respectively.

$k \quad$ high reduced frequency parameter.

$L \quad$ combination of $\sigma_{i}$ terms required for finding $\varrho^{1}$.

$M \quad$ Mach number of the steady flow with $M(\psi=0)=M_{0}$ and $M(\psi \rightarrow \pm \infty)=M_{\infty}$.

$N_{i} \quad$ perturbation functions to the governing equations generated by the transformation into $(\phi, \psi)$-space.

$p \quad$ unsteady pressure with Fourier transform $P(\alpha, \psi)$.

$q \quad$ steady perturbation to the mean velocity caused by the presence of the aerofoil, so $\boldsymbol{U}=\left(U_{0}+\epsilon q\right) \boldsymbol{e}_{\phi}$

$\tilde{Q} \quad$ function arising from the incident gust.

$s \quad$ scaling factor arising from the stretching of ray tubes through a shear flow.

$u \quad \phi$-component of unsteady velocity, with Fourier transform $U(\alpha, \psi)$.

$\boldsymbol{U} \quad$ steady parallel shear velocity, which takes value $U_{0}(\psi) \boldsymbol{e}_{\phi}$ far upstream, and $U_{0}^{*} \rightarrow$ $U_{\infty}^{*}$ as $\psi \rightarrow \pm \infty$.

$v \quad \psi$-component of unsteady velocity with Fourier transform $V(\alpha, \psi)$.

$Z$ triple of Fourier transformed solutions to the governing equations, $=\{P, U, V\}$.

$\alpha \quad$ Fourier transform variable of $\phi, \alpha=k \beta$ in the inner region.

$\beta^{s} \quad$ point of stationary phase of $\lambda$, taking the values $\beta_{0, \infty}$ when $M=M_{0, \infty}$.

$\Delta p \quad$ pressure jump across the trailing edge.

$\epsilon \quad$ small aerofoil thickness parameter.

$\lambda$ acoustic phase function in the inner region, and the first order behaviour of the phase in the outer region.

$\eta \quad$ inverse of $k / M(\psi)$, and used as an integration variable to construct solutions.

$\Omega \quad$ mean vorticity.

$\tilde{\Omega} \quad$ arbitrary upstream vorticity distribution of the incident disturbance. 
$\varrho \quad$ acoustic phase functions in the outer region.

$\phi \quad$ pseudo-velocity potential for the steady flow around the aerofoil, which in the inner region is $\Phi=k \phi$.

$\rho \quad$ unsteady density $\rho=M_{\infty}^{2} p$.

$\psi \quad$ streamfunction for the steady flow around the aerofoil, which in the inner region is $\Psi=k \psi$.

$\sigma_{i} \quad$ collection of perturbation terms to the governing equations, dependent on the $N_{i}$ and $q$, with Fourier transform $\Sigma_{i}(\alpha, \psi)$.

$\omega \quad$ hydrodynamic frequency such that $k=\omega M_{\infty}$.

$\zeta \quad$ factor required for the metric element, $h_{1}$, in $(\phi, \psi)$-space.

1 subscript denotes linearly independent solution from the outgoing wake-like solution.

a subscript denotes scattered acoustic solution.

$g \quad$ subscript denotes gust solution.

out subscript denotes outgoing wave-like behaviour.

$0 \quad$ superscript denotes leading-order term.

1 superscript denotes second-order perturbation term. 


\section{Appendix A}

From (3.3.1.2) we know that $A(\alpha) P_{\text {out }}(\alpha, 0)$ is analytic in the lower half $\alpha$ plane. Denote such a function by a _ $_{-}$, i.e. $A(\alpha) P_{\text {out }}(\alpha, 0)=\left[A(\alpha) P_{\text {out }}(\alpha, 0)\right]_{-}$. If we take an arbitrary minus function, $\kappa(\alpha)_{-}$, then

$$
A(\alpha) P_{\text {out }}(\alpha, 0) \kappa(\alpha)_{-}=\left[A(\alpha) P_{\text {out }}(\alpha, 0) \kappa(\alpha)_{-}\right]_{-}=G(\alpha)_{-} .
$$

We demand that $\kappa(\alpha)_{-}$has algebraic behaviour at infinity, and that $G(\alpha)_{ \pm}$vanishes at infinity. As before denote the $O(1)$ term of any function by a superscript ${ }^{0}$ and the $O(\epsilon \sqrt{k})$ term by a superscript ${ }^{1}$.

Define

$$
F(\alpha)_{-}=-\frac{1}{2 \pi k} \int_{0}^{\infty} \mathrm{e}^{-\mathrm{i} \alpha \Phi / k} v_{g}(\Phi / k, 0) d \Phi=F^{0}(\alpha)_{-}+\epsilon \sqrt{k} F^{1}(\alpha)_{-}
$$

SO

$$
A(\alpha) V_{\text {out }}(\alpha, 0)=F(\alpha)_{-}+F(\alpha)_{+},
$$

where $F(\alpha)_{+}$is analytic in the upper half $\alpha$ plane, and is unknown. Expanding (A.1) and (A.3) to $O(\epsilon \sqrt{k})$ and equating at each power yields

$$
\begin{aligned}
P_{\text {out }}^{0}(\alpha, 0) \kappa^{0}(\alpha)_{-} A^{0}(\alpha) & =G^{0}(\alpha)_{-}, \\
A^{0}(\alpha) V_{\text {out }}^{0}(\alpha, 0) & =F^{0}(\alpha)_{-}+F^{0}(\alpha)_{+},
\end{aligned}
$$

to leading order, and

$$
\begin{gathered}
P_{\text {out }}^{0}(\alpha, 0)\left[\kappa^{1}(\alpha)_{-} A^{0}(\alpha)+\kappa^{0}(\alpha)_{-} A^{1}(\alpha)\right]+P_{\text {out }}^{1}(\alpha, 0) \kappa^{0}(\alpha)_{-} A^{0}(\alpha)=G^{1}(\alpha)_{-}, \\
A^{1}(\alpha) V_{\text {out }}(\alpha, 0)+A^{0}(\alpha) V_{\text {out }}^{1}(\alpha, 0)=F^{1}(\alpha)_{-}+F^{1}(\alpha)_{+},
\end{gathered}
$$

to $O(\epsilon \sqrt{k})$. By demanding that $\kappa^{0}(\alpha)_{-}$satisfies

$$
\frac{P_{\mathrm{out}}^{0}(\alpha, 0)}{V_{\mathrm{out}}^{0}(\alpha, 0)}=\frac{\kappa^{0}(\alpha)_{+}}{\kappa^{0}(\alpha)_{-}},
$$

we find that

$$
G^{0}(\alpha)_{-}=\left[F^{0}(\alpha)_{-} \kappa^{0}(\alpha)_{+}\right]_{-} .
$$

This determines $G^{0}(\alpha)_{-}$and also $\kappa^{0}(\alpha)_{-}$from known quantities, $P$ and $V$. Hence

$$
A^{0}(\alpha)=\frac{G^{0}(\alpha)_{-}}{\kappa^{0}(\alpha)_{-} P_{\text {out }}(\alpha, 0)}
$$


is determined.

To next order

$G^{0}(\alpha)_{-}\left[\frac{P_{\text {out }}^{1}(\alpha, 0)}{P_{\text {out }}^{0}(\alpha, 0)}-\frac{V_{\text {out }}^{1}(\alpha, 0)}{V_{\text {out }}^{0}(\alpha, 0)}+\frac{\kappa^{1}(\alpha)_{-}}{\kappa^{0}(\alpha)_{-}}\right]+\kappa^{0}(\alpha)_{+} F^{1}(\alpha)_{+}+\kappa^{0}(\alpha)_{+} F^{1}(\alpha)_{-}=G^{1}(\alpha)_{-}$.

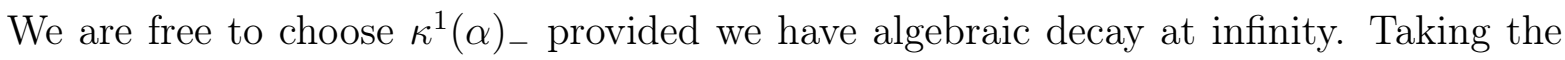
simplest case of $\kappa^{1}(\alpha)_{-}=0$ gives

$$
G^{1}(\alpha)_{-}=\left[\kappa^{0}(\alpha)_{+} F^{1}(\alpha)_{-}\right]_{-}+\left[G^{0}(\alpha)_{-}\left(\frac{P_{\text {out }}^{1}(\alpha, 0)}{P_{\text {out }}^{0}(\alpha, 0)}-\frac{V_{\text {out }}^{1}(\alpha, 0)}{V_{\text {out }}^{0}(\alpha, 0)}\right)\right]_{-},
$$

SO

$$
A^{1}(\alpha)=\frac{G^{1}(\alpha)_{-}-P_{\text {out }}^{1}(\alpha, 0) \kappa^{0}(\alpha)_{-} A^{0}(\alpha)}{P_{\text {out }}^{0}(\alpha, 0) \kappa^{0}(\alpha)_{-}} .
$$

We see a priori that our condition of $G$ vanishing at infinity is satisfied. Goldstein $(1978 a)$ proves that $G^{0}$ vanishes at infinity, and hence we only require that $\left[\kappa^{0}(\alpha)_{+} F^{1}(\alpha)_{-}\right]_{-}$ tends to zero as $\alpha \rightarrow \infty$. This is immediate if $G^{0}(\alpha) \rightarrow 0$ as $\alpha \rightarrow \infty$, since $F^{1}$ behaves asymptotically in a similar way to $F^{0}$ given the form of the velocity terms $v_{g}^{0,1}$. We proceed using the same method as Goldstein (1978a); define $R^{i}(\alpha)$ for $i=0,1$ by

$$
v_{g}^{i}(\Phi / k, 0)=\int_{k / M_{0}}^{k / M_{\infty}} \mathrm{e}^{\mathrm{i} \alpha \Phi / k} \tilde{\Omega}(\eta(\alpha)) R^{i}(\alpha) \frac{d \eta(\alpha)}{d \alpha} d \alpha .
$$

Using (A.2) we see

$$
F^{i}(\alpha)_{-}=\lim _{\delta \rightarrow 0_{+}} \frac{1}{2 \pi \mathrm{i}} \int_{k / M_{0}}^{k / M_{\infty}} \frac{\tilde{\Omega}\left(\eta\left(\alpha^{\prime}\right)\right)}{\alpha^{\prime}-\alpha+i \delta} R^{i}\left(\alpha^{\prime}\right) \frac{d \eta\left(\alpha^{\prime}\right)}{d \alpha^{\prime}} d \alpha^{\prime} .
$$

Using the Plemelj formula (Ablowitz \& Fokas, 2003) we find

$$
F^{i}(\alpha)_{-}=F^{i}(\alpha)_{+}-\left(H\left(\alpha-k / M_{0}\right)-H\left(\alpha-k / M_{\infty}\right)\right) \tilde{\Omega}(\eta(\alpha)) R^{i}(\alpha) \frac{d \eta(\alpha)}{d \alpha},
$$

where $H$ is the Heaviside function and the $F^{i}(\alpha)_{+}$are bounded at infinity in the upper half plane for $i=0,1$. Using (A.6) we find

$$
G^{0}(\alpha)_{-}=\frac{1}{2 \pi \mathrm{i}} \int_{0}^{\infty} M(\eta) \frac{\kappa^{0}(k / M(\eta))_{+}}{k-\alpha M(\eta)} \tilde{\Omega}(\eta) \tilde{Q}^{0}(\eta) d \eta
$$

where $\tilde{Q}^{i}(\eta)=R^{i}(k / M(\eta))$. This is the solution presented in Goldstein (1978a), in 
which $k$ is taken to have a small positive imaginary part to ensure convergence of all the integrals (this imaginary part is set to zero at the end of the analysis).

The solution for $G^{1}(\alpha)_{-}$is more complicated, but is found using the same method; we have

$$
\begin{aligned}
G^{1}(\alpha)_{-}-G^{1}(\alpha)_{+}= & G^{0}(\alpha)_{-}\left(\frac{P^{1}(\alpha, 0)}{P^{0}(\alpha, 0)}-\frac{V^{1}(\alpha, 0)}{V^{0}(\alpha, 0)}\right) \\
& -\left(H\left(\alpha-k / M_{0}\right)-H\left(\alpha-k / M_{\infty}\right)\right) \tilde{\Omega}(\eta(\alpha)) R^{1}(\alpha) \frac{d \eta(\alpha)}{d \alpha}
\end{aligned}
$$

So

$$
\begin{aligned}
G^{1}(\alpha)_{-}= & \frac{1}{2 \pi \mathrm{i}} \int_{0}^{\infty} M(\eta) \frac{\kappa^{0}(k / M(\eta))_{+}}{k-\alpha M(\eta)} \tilde{\Omega}(\eta) \tilde{Q}^{1}(\eta) d \eta \\
& +\frac{1}{2 \pi \mathrm{i}} \int_{0}^{\infty} \frac{k M^{\prime}(\eta) G^{0}(k / M(\eta))_{-}}{M(\eta)(k-\alpha M(\eta))}\left(\frac{P^{1}(k / M(\eta), 0)}{P^{0}(k / M(\eta), 0)}-\frac{V^{1}(k / M(\eta), 0)}{V^{0}(k / M(\eta), 0)}\right) d \eta .
\end{aligned}
$$




\section{Appendix B}

Here we present the Wiener-Hopf solutions to (3.6.1.2) and (3.6.1.3). Note that we need only find $A_{p}(\alpha)$ correct to leading order, since the pressure jump of the leading-edge field across the aerofoil, $\Delta p$, is an order of $\sqrt{k}$ smaller than the leading-edge pressure field, $p_{a}^{0}$. We have

$$
\begin{aligned}
& p_{a, H}^{0}\left(\Phi_{t}, \Psi_{t}\right)=-\operatorname{sgn}\left(\Psi_{t}\right) \int_{0}^{\infty} \frac{\tilde{\Omega}\left(\eta_{t}\right) \tilde{Q}^{0}\left(\eta_{t}\right) M\left(\eta_{t}\right)}{2 \pi \mathrm{i}} \\
& \int_{-\infty}^{\infty} \frac{\mathrm{e}^{\mathrm{i} \alpha \Phi_{t} / k} \kappa_{t}^{0}(\alpha)_{-} P_{\text {out }}^{0}\left(\alpha,\left|\Psi_{t}\right| / k\right)}{\left(k-\alpha M\left(\eta_{t}\right)\right) \kappa_{t}^{0}\left(k / M\left(\eta_{t}\right)\right)_{-} V_{\text {out }}^{0}(\alpha, 0)} d \alpha d \eta_{t} \\
& p_{a, H}^{1}\left(\Phi_{t}, \Psi_{t}\right)=-\operatorname{sgn}\left(\Psi_{t}\right) \int_{0}^{\infty} \frac{\tilde{\Omega}\left(\eta_{t}\right) \tilde{Q}^{0}\left(\eta_{t}\right) M\left(\eta_{t}\right)}{2 \pi \mathrm{i}}\{ \\
& \int_{-\infty}^{\infty} \frac{\mathrm{e}^{\mathrm{i} \alpha \Phi_{t} / k} \kappa_{t}^{0}(\alpha)_{-} P_{\text {out }}^{1}\left(\alpha,\left|\Psi_{t}\right| / k\right)}{\left(k-\alpha M\left(\eta_{t}\right)\right) \kappa_{t}^{0}\left(k / M\left(\eta_{t}\right)\right)_{-} V_{\text {out }}^{0}(\alpha, 0)} d \alpha d \eta_{t} \\
& \left.-\int_{-\infty}^{\infty} \frac{\mathrm{e}^{\mathrm{i} \alpha \Phi_{t} / k} P_{\text {out }}^{0}\left(\alpha,\left|\Psi_{t}\right| / k\right) \kappa_{t}^{0}(\alpha)_{-}}{\left(k-\alpha M\left(\eta_{t}\right)\right) \kappa_{t}^{0}\left(k / M\left(\eta_{t}\right)\right)_{-} V_{\text {out }}^{0}(\alpha, 0)} \frac{P_{\text {out }}^{1}(\alpha, 0)}{P_{\text {out }}^{0}(\alpha, 0)} d \alpha d \eta_{t}\right\} \\
& -\operatorname{sgn}\left(\Psi_{t}\right) \int_{0}^{\infty} \frac{\tilde{\Omega}\left(\eta_{t}\right) \tilde{Q}^{1}\left(\eta_{t}\right) M\left(\eta_{t}\right)}{2 \pi \mathrm{i}} \int_{-\infty}^{\infty} \frac{\mathrm{e}^{\mathrm{i} \alpha \Phi_{t} / k} P_{\text {out }}^{0}\left(\alpha,\left|\Psi_{t}\right| / K\right) \kappa_{t}^{0}(\alpha)-}{\left(k-\alpha M\left(\eta_{t}\right)\right) \kappa_{t}^{0}\left(k / M\left(\eta_{t}\right)\right)_{-} V_{\text {out }}^{0}(\alpha, 0)} d \alpha d \eta_{t} \\
& -\operatorname{sgn}\left(\Psi_{t}\right) \int_{0}^{\infty} \frac{k M^{\prime}\left(\eta_{t}\right) G_{t}^{0}\left(k / M\left(\eta_{t}\right)\right)_{+}}{2 \pi \mathrm{i} M\left(\eta_{t}\right)}\left(\frac{P_{\mathrm{out}}^{1}\left(k / M\left(\eta_{t}\right), 0\right)}{P_{\mathrm{out}}^{0}\left(k / M\left(\eta_{t}\right), 0\right)}-\frac{V_{\mathrm{out}}^{1}\left(k / M\left(\eta_{t}\right), 0\right)}{V_{\mathrm{out}}^{0}\left(k / M\left(\eta_{t}\right), 0\right)}\right) \\
& \int_{-\infty}^{\infty} \frac{\mathrm{e}^{\mathrm{i} \alpha \Phi_{t} / k} P_{\mathrm{out}}^{0}\left(\alpha,\left|\Psi_{t}\right| / k\right) \kappa_{t}^{0}(\alpha)_{-}}{\left(k-\alpha M\left(\eta_{t}\right)\right) \kappa_{t}^{0}\left(k / M\left(\eta_{t}\right)\right)_{-} V_{\text {out }}^{0}(\alpha, 0)} d \alpha d \eta_{t} \\
& p_{a, p}^{0}\left(\Phi_{t}, \Psi_{t}\right)=\int_{-\infty}^{\infty} \mathrm{e}^{\mathrm{i} \alpha \Phi_{t} / k} \frac{\kappa_{t}^{0}(\alpha)_{-} G_{t, p}(\alpha)_{-}}{V_{\text {out }}^{0}(\alpha, 0)} P_{\text {out }}^{0}\left(\alpha,\left|\Psi_{t}\right| / k\right) d \alpha
\end{aligned}
$$

where

$$
\begin{aligned}
\kappa_{t}^{0}(\alpha)_{+} \kappa_{t}^{0}(\alpha)_{-} & =\frac{V_{\text {out }}^{0}(\alpha, 0)}{P_{\text {out }}^{0}(\alpha, 0)} \\
G^{0}(\alpha)_{+} & =-\frac{1}{2 \pi \mathrm{i}} \int_{0}^{\infty} \frac{M\left(\eta_{t}\right) \tilde{\Omega}\left(\eta_{t}\right) \tilde{Q}\left(\eta_{t}\right)}{\left(k-\alpha M\left(\eta_{t}\right)\right) \kappa_{t}^{0}\left(k / M\left(\eta_{t}\right)\right)_{-}} d \eta_{t} \\
G_{t, p}(\alpha)_{-} & =\left[\kappa_{t}^{0}(\alpha)_{+} F_{t}(\alpha)_{-}\right]_{-}, \\
F_{t}(\alpha)_{-} & =-\frac{1}{2 \pi k} \int_{0}^{\infty} \Delta p\left(\Phi_{t} / k\right) \mathrm{e}^{-\mathrm{i} \alpha \Phi_{t} / k} d \Phi_{t} .
\end{aligned}
$$

Here $\eta_{t}$ is the trailing-edge version of the variable $\eta$ given in (3.2.0.5). 


\section{Chapter 4}

\section{Leading-Edge Stagnation-Point Noise Generated by Turbulence in Subsonic Uniform Flow}

As illustrated in Chapter 2, Section 2.8.2, the unsteady pressure on the nose of an aerofoil during gust-aerofoil interaction in uniform flow was predicted to be singular, which violates the small perturbation assumption, and hence the asymptotic results become invalid in a small region close to the nose of the aerofoil. In this chapter we therefore investigate the behaviour of the leading-edge stagnation point which is a crucial factor concerning the singularity. Despite the stagnation point of the steady flow around a cambered aerofoil not necessarily lying exactly on the leading edge, it is indeed the stagnation point that causes the singularity at the leading edge in Chapter 2, because the true location of the stagnation point for a cambered aerofoil is an $O\left(\epsilon^{2}\right)$ distance from the leading edge, which is a negligible distance in the previous analysis. We must therefore construct a new solution in a region close to the body which does not shift the location of the stagnation point, and that correctly assesses the effects the stagnation point has on the nearby flow.

We consider the evolution and interaction of an arbitrary gust of frequency $k$ in a steady uniform flow past a thin elliptic cylinder, specifically concentrating on the region close to the nose of the body, and in the far field at small angles away from the incident stagnation-point streamline (which is also referred to as the zero-streamline). The thickness of the ellipse is parameterised by $\tau \ll 1$, but unlike Chapters 1, 2 and 3 we do not impose $\tau k=O(1)$ when $k \gg 1$. Instead we obtain the leading-order contribution to the acoustic solution that is dependent on a multiplicative combination of thickness and frequency by imposing a different relationship between $\tau$ and $k$. This ensures that the thickness parameter can remain present in the analysis even to leading order, and we can investigate an ellipse whose thickness is not as firmly constrained as the previous requirement of $\tau k=O(1)$. In fact, in this chapter we not only consider high-frequency asymptotics but also low-frequency results.

To obtain the steady mean flow around an elliptic cylinder, the Joukowski transfor- 
mation is applied to uniform flow around a circular cylinder. The cylinders are assumed infinite in the spanwise direction so we again work essentially in two dimensions, but allow for upstream turbulent perturbations with spanwise velocity and phase components. We work with an ellipse to simplify the algebra as much as possible and because an ellipse and a parabolic-nosed body (such as the NACA 4-digit series aerofoils) both have radii of curvature proportional to thickness squared. Furthermore, for small angles of $O\left(\tau^{2}\right)$ from the leading-edge stagnation point, the difference between elliptic and parabolicnosed bodies, both with thickness $\tau$, is $O\left(\tau^{4}\right)$. During the analysis in this chapter we find that $O\left(\tau^{2}\right)$ angles are indeed those considered for the size of the region close to the stagnation point that we wish to study, and that terms of size $O\left(\tau^{4}\right)$ are negligible. Therefore to the orders retained in the analysis of this chapter, for the very small region close to the leading-edge stagnation point, a parabolic-nosed body can be approximated by an elliptic body. Crucially, $O\left(\tau^{2}\right)$ is non-negligible in this chapter, which is a significant difference from the work in the previous chapters, where we neglected terms of size thickness squared. By neglecting terms of size thickness squared in Chapter 2, we did not evaluate the effects of the stagnation point correctly and this led to the singularity in acoustic pressure at the leading edge of the aerofoil.

The analysis of an individual gust again follows Goldstein's rapid distortion theory (Goldstein, 1978b); we decompose the perturbation velocity into two parts, one that represents the evolution of the incident gust in the background flow, denoted by $\boldsymbol{u}^{(I)}$, and the other which contains all information about the interaction of the gust with the solid surface, denoted by $\boldsymbol{\nabla} \phi$, which contains all the acoustic pressure perturbations. We determine the evolution of any single gust of high or low frequency (denoted by $k$ ) in the background flow at points close to the nose of the ellipse and in the far field at small angles away from the zero-streamline.

From the analysis of a gust of arbitrary frequency, the effects of weak homogeneous, isotropic turbulence, incident from far upstream of a thin elliptic cylinder in uniform plane flow can be determined, since turbulence of this type can be decomposed into a Fourier series of gusts (Hunt, 1973). When turbulence is present in steady uniform flow past a bluff body, the stagnation point of the uniform flow causes great deformation of vortex tubes, hence our analysis focuses on the region close to the incident stagnation point where the vortical perturbations are most deformed, and previously this predicted a singularity in the unsteady pressure on the surface. The effects of the turbulence are assessed by integrating the velocity correlations due to individual Fourier components (single gusts) using a given upstream turbulent spectrum. We choose the upstream spectrum to be the von Kármán turbulent spectrum so that we can compare our re- 
sults with Goldstein (1978b), Durbin (1978) and Hunt (1973). This choice also ensures that the Kolmogorov "-5/3 law" (Kolmogorov, 1941), relating the energy density of the turbulence to its frequency, holds. The one-dimensional pressure spectrum is found as a function of spatial position and turbulent frequency. Both high and low reduced frequency (denoted by $k_{1} l$ where $k_{1} \propto k$ is the horizontal wavenumber, and $l$ is a typical integral lengthscale) turbulent pressure spectra are found and these are compared to experimental results. The far-field high and low reduced frequency limits of the turbulent pressure spectrum are found for regions very close to the frontal stagnation point of the ellipse, and also in a thin wedge around the zero-streamline far from the body. These solutions can be asymptotically extended outside these regions (as if using a matching theory). We can therefore compare the new far-field results found in this chapter to far-field results from Chapter 2. The two results, under appropriate asymptotic limits, have similar cylindrical acoustic pressure forms, hence we anticipate that the solution constructed in the "inner-inner" region in this chapter provides the required correction to the singular pressure point on the surface of the aerofoils considered in Chapter 2.

Unlike previous chapters, the analysis here is carried out in physical space, denoted by $(x, y)$ in Cartesian coordinates, and $(r, \theta)$ in polar coordinates. The elliptic cylinder is aligned with the uniform flow, so there is zero angle of attack, and the stagnation point of the base flow occurs precisely on the nose of the elliptic cylinder. We assume the Mach number is small (the definition of "small" is to be discussed later) hence we can take compressibility to be a perturbation on incompressibility (Van Dyke, 1975). In Section 4.1 we construct the stream and drift functions for uniform flow around an arbitrarily thin elliptic cylinder by using the Joukowski transformation and the corresponding functions for the flow around a circular cylinder which are well known. The stream and drift functions are required for evaluating the evolution of a single gust component in the background uniform flow which is done in Section 4.2. We also solve for the modified velocity potential, $\phi$, that contains all knowledge of the interaction of the gust with the solid surface. We construct solutions in both high- and low-frequency limits. The effects of homogeneous isotropic turbulence are investigated in Section 4.3, where high and low reduced frequency approximations are constructed for the one-dimensional turbulent pressure spectrum both close to the nose of the ellipse, and in the far field close to the zero-streamline. We compare our results with experimental data and other asymptotic analysis in Section 4.4, and conclusions are discussed in Section 4.5. 


\subsection{Streamfunction and Drift Function}

To obtain the flow field around an elliptic cylinder we conformally map from steady flow, with velocity $\boldsymbol{U}_{\infty}^{*}=U_{\infty}^{*} \hat{\boldsymbol{e}}_{x}$ far upstream, around a circular cylinder of radius $L^{*}$ using the Joukowski transformation. We non-dimensionalise velocities with respect to $U_{\infty}^{*}$, and lengths with respect to $L^{*}$.

\subsubsection{Streamfunction}

The velocity potential of the flow around the circular cylinder is

$$
\Phi(\varrho, \varphi)=\Phi_{0}+M^{2} \Phi_{1}+O\left(M^{4}\right)
$$

where $(\varrho, \varphi)$ are plane polar coordinates for the circle whose centre is taken as the origin, and we take compressibility as a perturbation to incompressibility (Van Dyke, 1975) by supposing the Mach number is small. We treat the uniform flow as essentially two-dimensional, allowing for only unsteady perturbations in the third dimension. We also assume the fluid is a perfect gas, hence for plane flow, $\Phi$, satisfies

$$
\begin{aligned}
\Phi_{\varrho \varrho}+\frac{\Phi_{\varrho}}{\varrho}+\frac{\Phi_{\varphi \varphi}}{\varrho^{2}}= & \frac{M^{2}}{2}\left[\left(\Phi_{\varrho} \frac{\partial}{\partial \varrho}+\frac{\Phi_{\varphi}}{\varrho^{2}} \frac{\partial}{\partial \varphi}\right)\left(\Phi_{\varrho}^{2}+\frac{\Phi_{\varphi}^{2}}{\varrho^{2}}\right)\right. \\
& \left.+(\gamma-1)\left(\Phi_{\varrho}^{2}+\frac{\Phi_{\varphi}^{2}}{\varrho^{2}}-1\right)\left(\Phi_{\varrho \varrho}+\frac{\Phi_{\varrho}}{\varrho}+\frac{\Phi_{\varphi \varphi}}{\varrho^{2}}\right)\right]
\end{aligned}
$$

where $\gamma$ is the ratio of specific heats which is constant. Solving for successive powers of $M^{2}$ yields

$$
\begin{aligned}
& \Phi_{0}(\varrho, \varphi)=\left(\varrho+\frac{1}{\varrho}\right) \cos \varphi \\
& \Phi_{1}(\varrho, \varphi)=\left(\frac{13}{12 \varrho}-\frac{1}{2 \varrho^{3}}+\frac{1}{12 \varrho^{5}}\right) \cos \varphi+\left(\frac{1}{12 \varrho^{3}}-\frac{1}{4 \varrho}\right) \cos 3 \varphi
\end{aligned}
$$

which is as found by Van Dyke (1975). With this, we can find the velocity field, $\boldsymbol{U}=\boldsymbol{\nabla} \Phi$, and hence the streamfunction, $\Psi$, defined by

$$
\left(\begin{array}{c}
U_{\varrho} \\
U_{\varphi}
\end{array}\right)=\left(\begin{array}{c}
\frac{1}{\rho} \frac{1}{\varrho} \frac{\partial \Psi}{\partial \varphi} \\
-\frac{1}{\rho} \frac{\partial \Psi}{\partial \varrho}
\end{array}\right) .
$$

Here $\rho$ is the density (non-dimensionalised with respect to the density far upstream, $\left.\rho_{\infty}^{*}\right)$, which is solved for via the continuity equation or the equation of state for an ideal 
isothermic gas (Sakurai \& Arai, 1981);

$$
\rho=\left(1-\frac{\gamma-1}{2} M^{2}\left(|q|^{2}-1\right)\right)^{\frac{1}{\gamma-1}}
$$

where $q$ is the complex velocity equal to $U_{x}-\mathrm{i} U_{y}$ in Cartesian coordinates. Expanding in powers of $M^{2}$ yields

$$
\rho=1+M^{2}\left(\frac{1}{\varrho^{2}} \cos 2 \theta-\frac{1}{2 \varrho^{4}}\right)+O\left(M^{4}\right)
$$

hence

$$
\Psi(\varrho, \varphi)=\left(\varrho-\frac{1}{\varrho}\right) \sin \varphi+\frac{M^{2}}{\varrho^{4}}\left(\varrho-\frac{1}{\varrho}\right) \sin \varphi\left(6 \varrho^{2} \cos 2 \varphi-4 \varrho^{2}-1\right)+O\left(M^{4}\right) .
$$

\subsubsection{Joukowski Transformation of Coordinates}

We have calculated the streamfunction and hence velocity field of the flow around a circular cylinder. We apply the Joukowski transformation,

$$
\zeta=z+\frac{b^{2}}{z}
$$

to map this to the flow around an elliptic cylinder, where $\zeta=\varrho \mathrm{e}^{\mathrm{i} \varphi}$, and $z=r \mathrm{e}^{\mathrm{i} \theta}$ defines $(r, \theta)$ polar coordinates for the elliptic cylinder geometry (centred on the centre of the ellipse). The unit circular cylinder is mapped conformally to an elliptic cylinder of minor axis $1-b^{2}$ and major axis $1+b^{2}$. The thickness ratio, $\tau=\left(1-b^{2}\right)\left(1+b^{2}\right)^{-1}$, is therefore less than unity and by choosing $b$ sufficiently close (but not equal) to one, we obtain an arbitrarily thin ellipse. The transformation of coordinates is given by

$$
\begin{aligned}
\varrho & =\frac{1}{2 \cos \varphi}\left(r \cos \theta-\sqrt{r^{2} \cos ^{2} \theta-4 b^{2} \cos ^{2} \varphi}\right) \\
\sin ^{2} \varphi & =\frac{4 b^{2}-r^{2}}{8 b^{2}}+\frac{1}{2} \sqrt{\frac{\left(r^{2}-4 b^{2}\right)^{2}}{16 b^{4}}+\frac{r^{2} \sin ^{2} \theta}{b^{2}}}
\end{aligned}
$$

Signs are chosen to ensure that the stagnation point at the leading edge remains at the leading edge. We expand for $\theta$ close to $\pi$ which maps to $\varphi$ close to $\pi$, therefore near the leading-edge stagnation point the coordinate transformation is given by

$$
\varrho \approx \frac{1}{2}\left(\sqrt{r^{2}-4 b^{2}}+r\right)+O\left((\pi-\theta)^{2}\right),
$$




$$
\sin \varphi \approx \frac{r \sin \theta}{\sqrt{r^{2}-4 b^{2}}}+O\left((\pi-\theta)^{2}\right)
$$

This approximation for $\sin \varphi$ is valid provided $\sin \theta /\left(1-b^{2}\right) \ll 1$, hence we must restrict the size of $\theta$ and $\tau$ so that this holds. Both $\theta$ and $\tau$ are scaled appropriately with the frequency of the incident gust, $k$, later.

\subsubsection{Drift Function}

The non-dimensionalised drift function is defined by Lighthill (1956) as

$$
\Delta=\int_{-\infty}^{x}\left(\frac{1}{U_{x^{\prime}}}-1\right) d x^{\prime}
$$

which relates to the difference between the time taken for any fluid particle to travel from far upstream to position $x$ along a streamline and the time taken to do so in uniform flow, and is the Cartesian equivalent to $g$ given in (2.1.0.4g). The drift function relates to time via $t=x+\Delta(x)$. In polar coordinates it is easiest to find the drift function by integrating the relation

$$
d t=\frac{r d \theta}{U_{\theta}}
$$

To evaluate (4.1.3.2) we require an expansion of $r$ in terms of $\theta$ along a given streamline. Since we wish to investigate the effect of the stagnation point of the flow we consider a streamline close to the zero-streamline, i.e. $\psi(r, \theta)=\delta$ for $0<\delta \ll 1$, and write

$$
\delta=\Psi_{0}(r, \theta)+M^{2} \Psi_{1}(r, \theta) .
$$

We solve (4.1.3.3) for $r$ by writing

$$
r=r_{0}+\delta r_{1}+\delta^{2} r_{2}+M^{2} r_{4}+\delta M^{2} r_{3}+O\left(\delta^{3}, M^{4}, \delta^{2} M^{2}\right)
$$

similarly to Lighthill (1956). Equating (4.1.3.3) at each order using (4.1.3.4) yields solutions for the $r_{i}$ which are given by

$$
\begin{aligned}
& r_{0}=1+b^{2}, \\
& r_{1}=\frac{\left(1-b^{2}\right)^{2}}{2\left(1+b^{2}\right)} \csc \theta, \\
& r_{2}=\frac{\left(1-b^{2}\right)^{2}}{8\left(1+b^{2}\right)^{3}}\left(b^{4}+10 b^{2}+1\right) \csc ^{2} \theta,
\end{aligned}
$$




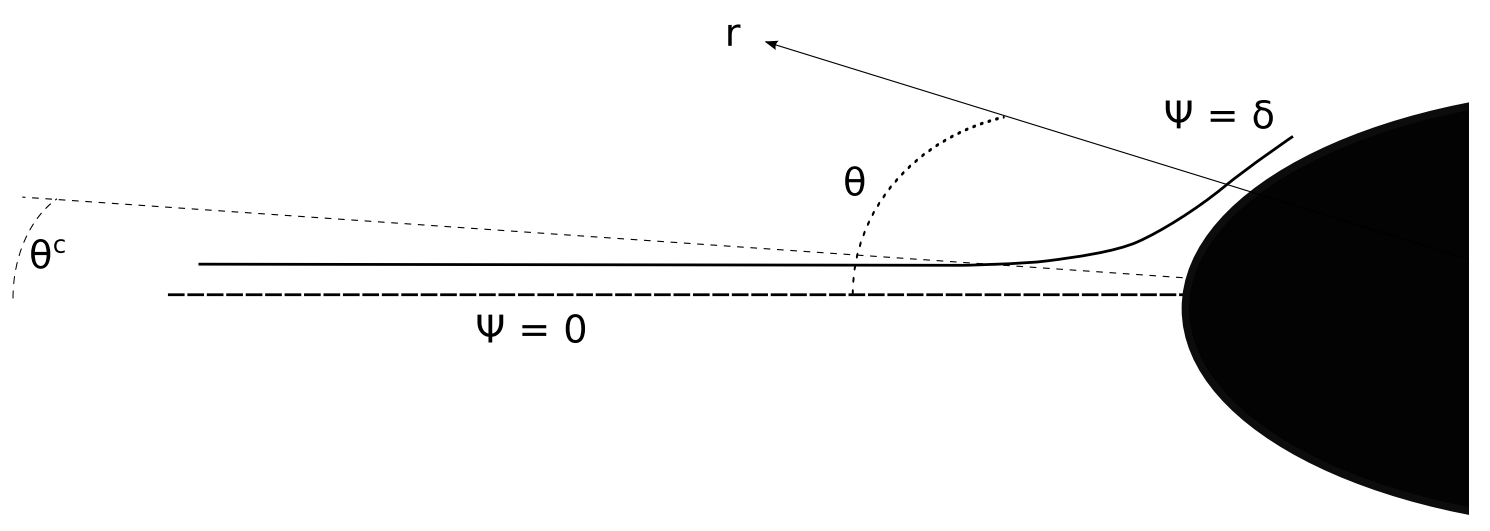

Figure 4.1: Diagram of the flow problem and coordinate system.

$$
\begin{aligned}
& r_{3}=\frac{\left(1+b^{2}\right)}{2} \sin \theta-\frac{\left(1-b^{2}\right)^{2}}{24\left(1+b^{2}\right)} \csc \theta \\
& r_{4}=0 .
\end{aligned}
$$

We notice that (4.1.3.4) is in fact a series in $\delta / \sin \theta$ so it is only valid when $1 \gg(\pi-\theta)>$ $\delta$. We further note that the $\delta$-streamline is approximately a straight line from upstream infinity, which arises from the uniform flow, followed by a curve around the ellipse. We can treat this in a similar manner to Goldstein (1978b); when calculating the drift we use a uniform flow approximation for the straight line section, yielding an additive constant to the drift function, and our expansion, (4.1.3.4), for the curved section. Since we only end up using derivatives of the drift function to analyse the interaction of a gust with the body, we do not explicitly evaluate the constant contribution from the uniform flow-like section.

We change our polar coordinate system to measure $\theta$ clockwise from the zero-streamline, as shown in Figure 4.1, so we can treat the region close to the stagnation point now as a region of small $\theta$. The definition of the "small- $\theta$ " region, wherein our asymptotic expansions are valid, can be found in Appendix A along with the derivation of the drift function close to the body. We denote by $\theta^{c}$ the angle at which we swap from the uniform flow approximation for the straight section of the streamline, to the curved section, illustrated in Figure 4.1. 


\subsection{Velocity Potential Given an Incident Gust}

Here we evaluate the evolution of an arbitrary gust in uniform flow around a thin ellipse. We take an unsteady harmonic disturbance upstream to be a generalised Fourier integral

$$
\boldsymbol{u}_{\infty}\left(\boldsymbol{x}-\hat{\boldsymbol{e}}_{x} t\right)=\int \boldsymbol{A}(\boldsymbol{k}) \mathrm{e}^{\mathrm{i} \boldsymbol{k} \cdot \boldsymbol{x}-k_{1} t} d \boldsymbol{k}
$$

We require $\boldsymbol{u}_{\infty}$ to be solenoidal so demand $\boldsymbol{A} \cdot \boldsymbol{k}=0$ for all $\boldsymbol{k}$. We write $\boldsymbol{k}=k \boldsymbol{k}^{\dagger}=$ $\left(k_{1}, k_{2}, k_{3}\right)$, where $k$ is our asymptotic frequency parameter, non-dimensionalised with respect to $L^{*-1}$, and $k_{i}^{\dagger}$ are wavevector components. For simplicity we initially consider just one Fourier mode. In particular the gust mode far upstream is

$$
\boldsymbol{u}_{\infty}\left(\boldsymbol{x}-\hat{\boldsymbol{e}}_{x} t\right)=\boldsymbol{A}(\boldsymbol{k}) \mathrm{e}^{\mathrm{i}\left[k_{1}(x-t)+k_{2} y+k_{3} z\right]}
$$

It follows from Goldstein (1978b) that the non-dimensionalised incident velocity components evolve as

$$
\begin{aligned}
& u_{r}^{(I)}=\left(A_{1} \frac{\Delta}{r}+A_{2} \frac{\Psi}{r}\right) \mathrm{e}^{\mathrm{i}\left[k_{1}(\Delta-t)+k_{2} \Psi+k_{3} z\right]}, \\
& u_{\theta}^{(I)}=\left(A_{1} \frac{1}{r} \frac{\partial \Delta}{\partial \theta}+A_{2} \frac{1}{r} \frac{\partial \Psi}{\partial \theta}\right) \mathrm{e}^{\mathrm{i}\left[k_{1}(\Delta-t)+k_{2} \Psi+k_{3} z\right]} \\
& u_{z}^{(I)}=A_{3} \mathrm{e}^{\mathrm{i}\left[k_{1}(\Delta-t)+k_{2} \Psi+k_{3} z\right]} .
\end{aligned}
$$

Supposing that the steady mean flow around the ellipse is $\boldsymbol{U}=\boldsymbol{U}_{0}+M^{2} \boldsymbol{U}_{1}+O\left(M^{4}\right)$, and $\boldsymbol{u}$ is the small velocity field generated by the perturbation $\boldsymbol{u}_{\infty}$ upstream to the uniform mean flow, then we can write

$$
\boldsymbol{u}=\boldsymbol{\nabla} \phi+\boldsymbol{u}^{(I)}
$$

where $\phi$ contains all knowledge of the interaction of the gust with the solid surface (and contains the acoustic part of the flow). The governing equation for $\phi$ is

$$
\begin{array}{r}
\frac{D_{0}}{D t}\left(\frac{1}{c^{2}} \frac{D_{0}}{D t}\right) \phi-\frac{1}{\rho} \boldsymbol{\nabla} \cdot(\rho \boldsymbol{\nabla} \phi)=\frac{1}{\rho} \boldsymbol{\nabla} \cdot\left(\rho \boldsymbol{u}^{(I)}\right), \\
\boldsymbol{\nabla} \phi \cdot \boldsymbol{n}=-\boldsymbol{u}^{(I)} \cdot \boldsymbol{n} \text { on the solid surface, }
\end{array}
$$

where $\frac{D_{0}}{D t}=\frac{\partial}{\partial t}+\boldsymbol{U} \cdot \boldsymbol{\nabla}$. This is the typical velocity decomposition for rapid distortion theory, resulting in the familiar acoustic equation (see (1.1.2.2)). Here $c$ is the non- 
dimensionalised speed of sound for the steady flow and is given by

$$
c^{2}=\frac{1}{M^{2}}\left[1-\frac{\gamma-1}{2} M^{2}\left(|q|^{2}-1\right)\right] \equiv M^{-2}\left(1+M^{2} c_{1}^{2}\right),
$$

where $q$ is the complex velocity for the steady flow around the ellipse.

Equation (4.2.0.3) allows us to write the source term of (4.2.0.5a) as

$$
\frac{1}{\rho} \boldsymbol{\nabla} \cdot\left(\rho \boldsymbol{u}^{(I)}\right)=\left(H_{0}+\mathrm{i} H_{1}\right) \mathrm{e}^{\mathrm{i}\left[k_{1}(\Delta-t)+k_{2} \Psi+k_{3} z\right]}
$$

where the $H_{i}$ are real functions defined by

$$
\begin{aligned}
H_{0}= & \frac{A_{1}}{r} \frac{\partial \Delta}{\partial r}+\frac{A_{2}}{r} \frac{\partial \Psi}{\partial r}+\frac{A_{1}}{r^{2}} \frac{\partial^{2} \Delta}{\partial \theta^{2}}+\frac{A_{2}}{r^{2}} \frac{\partial^{2} \Psi}{\partial \theta^{2}} \\
& +M^{2}\left[\left(\frac{A_{1}}{r} \Delta+\frac{A_{2}}{r} \Psi\right) \frac{\partial \varrho}{\partial r}\left(\frac{2}{\varrho^{5}}-\frac{2}{\varrho^{3}} \cos 2 \varphi\right)-\frac{2 \sin 2 \varphi}{r \varrho^{2}} \frac{\partial \varphi}{\partial \theta}\left(\frac{A_{1}}{r} \frac{\partial \Delta}{\partial \theta}+\frac{A_{2}}{r} \frac{\partial \Psi}{\partial \theta}\right)\right], \\
H_{1}= & A_{1} k_{1}\left[\left(\frac{1}{r} \frac{\partial \Delta}{\partial \theta}\right)^{2}+\frac{\partial \Delta}{\partial r} \frac{\Delta}{r}\right]+A_{2} k_{2}\left[\left(\frac{1}{r} \frac{\partial \Psi}{\partial \theta}\right)^{2}+\frac{\partial \Psi}{\partial r} \frac{\Psi}{r}\right]+A_{3} k_{3} \\
& +A_{1} k_{2}\left[\frac{1}{r^{2}} \frac{\partial \Delta}{\partial \theta} \frac{\partial \Psi}{\partial \theta}+\frac{\Delta a}{r} \frac{\partial \Psi}{\partial r}\right]+A_{2} k_{1}\left[\frac{1}{r^{2}} \frac{\partial \Delta}{\partial \theta} \frac{\partial \Psi}{\partial \theta}+\frac{\Psi}{r} \frac{\partial \Psi}{\partial r}\right] .
\end{aligned}
$$

\subsubsection{High-Frequency Solution}

We first suppose that the frequency of the gust is high, $k \gg 1$. We wish to solve (4.2.0.5a) in a region close to the stagnation point, hence we use the small- $\theta$ approximation (discussed in Appendix A), particularly for the conformal mapping (4.1.2.1d). Setting

$$
\phi_{i}=\mathrm{e}^{-\mathrm{i} k_{1} t+\mathrm{i} k_{3} z} \bar{\phi}_{i}
$$

and neglecting small terms in (4.2.0.5a) yields

$$
\bar{\phi}^{\prime \prime}-k^{2} w^{2} r^{2} \bar{\phi}=-r^{2} f(r, \theta)
$$

where ' denotes differentiation with respect to $\theta, w^{2}=k_{1}^{\dagger 2} M^{2}-k_{3}^{\dagger 2}$, and

$$
f(r, \theta)=\left(H_{0}(r, \theta)+\mathrm{i} H_{1}(r, \theta)\right) \mathrm{e}^{\mathrm{i} k_{1} \Delta(r, \theta)+\mathrm{i} k_{2} \Psi(r, \theta)}
$$

We suppose $w=O(1)$ with respect to $k$, and $w^{2}>0$, so the spanwise component of frequency, $k_{3}$, is much smaller than the streamwise component, $k_{1}$. The small- $\theta$ 
approximations of the source terms $H_{0,1}$ are dominated by $\theta$ derivatives of $\Delta$, yielding

$$
H_{0} \sim O\left(\frac{A_{1}}{a_{1} \theta^{2} r^{2}}\right), \quad H_{1} \sim O\left(\frac{A_{1} k_{1}}{a_{1}^{2} \theta^{2} r^{2}}\right)
$$

along a streamline, hence the terms in (4.2.1.2) balance if $\theta=O\left(k^{-1}\right)$. This defines the small- $\theta$ region given $k$, and is consistent with any previous restraints on the size of $\theta$ given in Appendix A. Initially this appears to yield the same sized leading-edge region as found in Chapter 2, however we stress that there are two key differences in this chapter. First, the thickness of the ellipse, $\tau$, is not constrained by the limit $\tau k=O(1)$, and secondly, throughout Chapter 2 we took $\theta=O(1)$ whereas now we have created a region in which $\theta$ scales with $k^{-1}$.

We solve (4.2.1.2) using a Green's function, imposing the homogeneous boundary conditions

$$
\begin{aligned}
G & =0 \text { at } \theta=\theta^{c}, \\
\frac{\partial G}{\partial \theta} & =0 \text { at } \theta=\theta^{c},
\end{aligned}
$$

and requiring

$$
G^{\prime \prime}\left(\theta, \theta^{\prime}, r, r^{\prime}\right)+\omega(r) G\left(\theta, \theta^{\prime}, r, r^{\prime}\right)=\delta^{f}\left(\theta-\theta^{\prime}\right) \frac{\delta^{f}\left(r-r^{\prime}\right)}{2 \pi r}
$$

where $\omega(r)=k^{2} w^{2} r^{2}$, and on the right hand side are Dirac delta functions, $\delta^{f}$. Since $\omega>$ 0 , the Green's function is oscillatory, hence on application of the method of stationary phase (Bender \& Orszag, 1978) we obtain a solution where $\bar{\phi}_{0}=O\left(k^{-3 / 2}\right)$. If $\omega<0$, we would obtain an exponentially decaying solution, which would be negligible compared to the oscillatory solution, thus we only consider $\left|k_{3}\right| \leq M\left|k_{1}\right|$. In this case the Green's function is given by

$$
G\left(\theta, \theta^{\prime}, r, r^{\prime}\right)=\left\{\begin{array}{cl}
0 & \text { if } \theta<\theta^{\prime} \\
\frac{1}{\sqrt{\omega(r)}} \sin \left[\sqrt{\omega(r)}\left(\theta-\theta^{\prime}\right)\right] \frac{\delta^{f}\left(r-r^{\prime}\right)}{2 \pi r} & \text { if } \theta>\theta^{\prime}
\end{array} .\right.
$$

We solve the homogeneous version of (4.2.1.2), satisfying zero normal velocity on the solid surface, separately to the non-homogeneous equation. Hence if superscript $h$ denotes the 
homogeneous solution and superscript $p$ the particular solution, then

$$
\bar{\phi}^{p}=\frac{r}{2 \pi k \sqrt{k_{1}^{\dagger 2} M^{2}-k_{3}^{\dagger 2}}} \int_{\theta}^{\epsilon}\left(H_{0}+\mathrm{i} H_{1}\right)\left(r, \theta^{\prime}\right) \mathrm{e}^{\mathrm{i} k\left(k_{1}^{\dagger} \Delta\left(r, \theta^{\prime}\right)+k_{3}^{\dagger} \Psi\left(r, \theta^{\prime}\right)\right)} \sin \left(k \Omega\left(\theta-\theta^{\prime}\right)\right) d \theta^{\prime},
$$

where $\omega=k^{2} \Omega^{2}$ and $\epsilon$ is the upper limit of validity of the small $-\theta$ solution for a given frequency. We notice that in the small- $\theta$ approximation, $\Psi=O(\delta)$, which we neglect (we discuss how $\delta$ scales with $k$ later, which ensures this assumption is valid). The phase functions in (4.2.1.8) are therefore $g_{ \pm}\left(r, \theta^{\prime}\right)=k_{1}^{\dagger} \Delta\left(r, \theta^{\prime}\right) \pm \Omega(r)\left(\theta-\theta^{\prime}\right)$. By approximating the drift function by the expression found along a streamline, (A.6a), we find the points of stationary phase of $g_{ \pm}$are given by

$$
\theta_{s}^{\prime} \approx \frac{ \pm\left(1+b^{2}\right) k_{1}^{\dagger}}{\Omega a_{1}} \mp M^{2} \frac{\left(a_{13}+3 a_{14}\right)\left(1+b^{2}\right) k_{1}^{\dagger}}{\Omega a_{1}^{2}}=\theta_{s}^{0}+M^{2} \theta_{s}^{1}
$$

Note, we are supposing that $k_{1}^{\dagger} M=O(1)$, but since we are working to leading order in $k$ only, $M^{2} \theta_{s}^{1} \ll \theta_{s}^{0}$, hence we neglect $M^{2} \theta_{s}^{1}$. We require $0<\theta<\theta_{s}^{\prime}<\epsilon$, hence on noting that $a_{1}<0$ (given in Appendix A) we see that only $g_{-}\left(r, \theta^{\prime}\right)$ yields a valid point of stationary phase, hence

$$
\bar{\phi}^{p} \sim \frac{-r}{4 \pi \mathrm{i} k^{3 / 2} \sqrt{k_{1}^{\dagger 2} M^{2}-k_{3}^{\dagger 2}}} f_{0}\left(r, \theta_{s}^{0}\right) \sqrt{\frac{2 \pi}{g^{\prime \prime}\left(r, \theta_{s}^{0}\right)}} \mathrm{e}^{\mathrm{i} \pi / 4} e^{\mathrm{i} k \Omega\left(\theta_{s}^{0}-\theta\right)} .
$$

We are permitted to use the streamline approximation of the drift function when using the method of stationary phase because the result we wish to obtain after the application of the method of stationary phase is one valid for a small- $\theta$ region close to the body, i.e. on and around the streamline. For small values of $\theta$, on the surface of the ellipse, $r \approx\left(1+b^{2}\right)+O\left(\theta^{2}\right)$, hence in a small region close to the body, for all values of $\theta$ we consider close to the stagnation point, $r$ varies from its value on the streamline only by an $O\left(\theta^{2}\right)$ amount, which is negligible. We do, however, write $r$ as a variable in most of the following functions to make it clear where it features in the solutions, rather than setting it to $1+b^{2}$ outright.

The homogeneous solution that solves for the solid boundary condition requires evaluation of the Green's function on the surface

$$
\bar{\phi}^{h}(r, \theta)=-\left.\int_{\delta}^{\epsilon} G\left(\theta^{\prime}, \theta, r^{\prime}, r\right) \frac{\partial \bar{\phi}^{h}}{\partial n}\right|_{r^{\prime}=\sqrt{\left(1+b^{2}\right)^{2} \cos ^{2} \theta^{\prime}+\left(1-b^{2}\right)^{2} \sin ^{2} \theta^{\prime}}} r^{\prime} d \theta^{\prime} .
$$


This expression is valid both on and off the streamline, hence we view $r$ and $\theta$ here as truly independent variables. Technically we should be integrating over the entire surface of the ellipse to capture all of the boundary condition behaviour, however our constructed Green's function and particular solution are only valid for $\theta \in[\delta, \epsilon]$. Beyond this region we can attempt to extend the solution, but we find that $\frac{\partial \bar{\phi}^{p}}{\partial n}$ is an order of $\sqrt{k}$ lower than within this region. Also, $\boldsymbol{u}^{(I)} \cdot \boldsymbol{n} \sim A_{1} \Delta / r$ on the surface, since $\Psi \ll 1$, and $\Delta \sim r \cos \theta$ when $\theta \rightarrow \pi / 2$, hence $\boldsymbol{u}^{(I)} . \boldsymbol{n}$ is also small. Thus the greatest contribution to $\bar{\phi}^{h}$ is from the small $-\theta$ region so this is all we consider. Evaluating the integral in (4.2.1.11) yields;

$$
\bar{\phi}^{h}(r, \theta)=\left.\frac{1}{2 \pi k R\left(\theta^{h} ; r\right) w} \sin \left[k R\left(\theta^{h} ; r\right) w\left(\theta-\theta^{h}(r)\right)\right] \frac{\partial \bar{\phi}^{h}}{\partial n}\right|_{\left(R\left(\theta^{h} ; r\right), \theta^{h}\right)},
$$

where

$$
\begin{aligned}
\theta^{h}(r) & =2 \arctan \left[\sqrt{\frac{1-6 b^{2}+b^{4}-r^{2}+4 b \sqrt{r^{2}-\left(1-b^{2}\right)^{2}}}{\left(1+b^{2}\right)^{2}-r^{2}}}\right], \\
R\left(\theta^{h} ; r\right) & =\sqrt{\left(1+b^{2}\right)^{2} \cos ^{2} \theta^{h}+\left(1-b^{2}\right)^{2} \sin ^{2} \theta^{h}}, \\
\frac{\partial \bar{\phi}^{h}}{\partial n}(r, \theta) & =-\overline{\boldsymbol{u}}^{(I)} \cdot \boldsymbol{n}-\frac{\partial \bar{\phi}^{p}}{\partial n} .
\end{aligned}
$$

From here on in we neglect any terms of $O\left(M^{2}\right)$, except those present in the form $k_{1}^{\dagger 2} M^{2}$, due to the complexity of the following calculations. We also treat terms of $O(\delta)$ as negligible.

The overall scaling of our particular solution is

$$
\bar{\phi}^{p} \sim k^{-3 / 2}\left(g^{\prime \prime}\left(\theta_{s}^{0}\right)\right)^{-1 / 2}\left(\theta_{s}^{0}\right)^{-2}\left[\frac{1}{a_{1}}+\frac{k}{a_{1}^{2}}\right] \sim k^{-3 / 2}\left(1-b^{2}\right)^{-1}\left[k\left(1-b^{2}\right)^{2}-1\right],
$$

which for a sufficient scaling of thickness results in the same order (in $k$ ) as our previous inner solution, (1.2.0.3), from Chapter 2. This required scaling is $\left(1-b^{2}\right)=O\left(k^{-1 / 2}\right)$, which we highlight implies that $O\left(\tau^{2}\right)$ terms are non-negligible. Note that this scaling also ensures that $H_{0}$ and $H_{1}$, from (4.2.1.4), are comparable. Given this scaling, for the series (A.5) to be valid we require $\theta<O\left(k^{-1 / 2}\right)$. This agrees with the limit given for the validity of (A.1b). We have not bounded $M$ yet, so we choose a scaling that permits a valid asymptotic series for $\bar{\phi}$. We want the scaling to ensure that (A.6a) is also an asymptotic series and hence choose $M=O\left(k^{-1}\right)$, which is consistent with the assumption that we neglect terms of $O\left(M^{2}\right)$. We are free to choose the scaling of $\delta$ provided it satisfies $\delta \ll k^{-2}$ which ensures that neglecting $\delta$ is justified. 


\section{Far Field Implications and Matchings to Previous Work}

We can consider what happens to this high-frequency "inner" solution as we take $r$ to be large, and maintain the small- $\theta$ approximation. For $r \gg 1,(4.2 .0 .5 \mathrm{a})$ has negligible source term (to the orders retained previously) hence (4.2.1.2) also has zero source term. By taking the limits of $r \gg 1$ and small $\theta$ we therefore find that $\phi^{p} \approx 0$, so we are left with $\phi \approx \phi^{h}$ as $r \rightarrow \infty$. We notice that $\theta^{h} \rightarrow \pi / 2$ for $r \rightarrow \infty$ hence it is out of range of the limits of integration, thus $\phi^{h} \rightarrow 0$ also. This tells us, to the orders retained in the calculations, the far-field noise is negligible in the small- $\theta$ region. This may initially seem incorrect in that there is negligible far-field sound radiated upstream from the leading edge, but recall we are only considering a thin wedge about the stagnation point. We could have anticipated this by considering the far-field result for an incident gust in uniform steady flow (Chapter 2); the far-field directivity of this function scales as $O\left((k r)^{-1 / 2} \theta\right)$, for $\theta$ measured clockwise from the zero-streamline direction. If $\theta=O\left(k^{-1}\right)$ this contribution is also deemed negligible in the analysis. In the next paragraph we see that this $\theta=O\left(k^{-1}\right)$ scaling holds, hence we have an agreement between the far-field results presented here, and those from previous work in Chapter 2 to first order in thickness. We can also consider Tsai (1992, Figure 4.25), and Figure 4.2 which visually show that directly upstream in the far-field, the acoustics are much smaller than elsewhere, hence to orders retained here would be deemed negligible. Note, the Mach number for Figure 4.2 is much larger than allowable in this chapter, however is sufficient to illustrate the lack of direct upstream radiation.

We can also consider $\phi^{p}$ as $\theta$ moves out of our predefined "small" region, and when $r$ is large. This asymptotically extends our solution into a leading-edge outer region similar to that described in Chapters 1 and 2. We use the method of stationary phase again, but this time do not restrict the drift function, $\Delta$, to its approximation along a streamline, since we have moved out of the region where (A.6a) is valid. Further, $\Delta$ is now seen as a function of both $r$ and $\theta$ and we take $\theta=O(1)$ but $\theta<\pi / 2$. The point of stationary phase, (4.2.1.9), now occurs at $k_{1}^{\dagger} \Delta^{\prime}\left(r, \theta_{s}\right)=\Omega \sim r$ hence $\Delta \sim r \cos \theta$. Therefore

$$
\bar{\phi}^{p} \sim \frac{r}{k} \frac{f\left(r, \theta_{s}\right)}{\sqrt{k \Delta^{\prime \prime}\left(r, \theta_{s}\right)}} \mathrm{e}^{\mathrm{i} k w r\left(\theta_{s}-\theta\right)}
$$

for large $k$. For $r \gg 1$, we see $f\left(r, \theta_{s}\right) \sim \frac{\Delta}{r^{2}} \sim \frac{\cos \theta}{r}$, which is physically sensible because the far field sees a dipole source created by the body (Gershfeld, 2004), and this yields

$$
\phi^{p} \sim \frac{\sqrt{\cos \theta}}{k^{3 / 2} \sqrt{r}}
$$




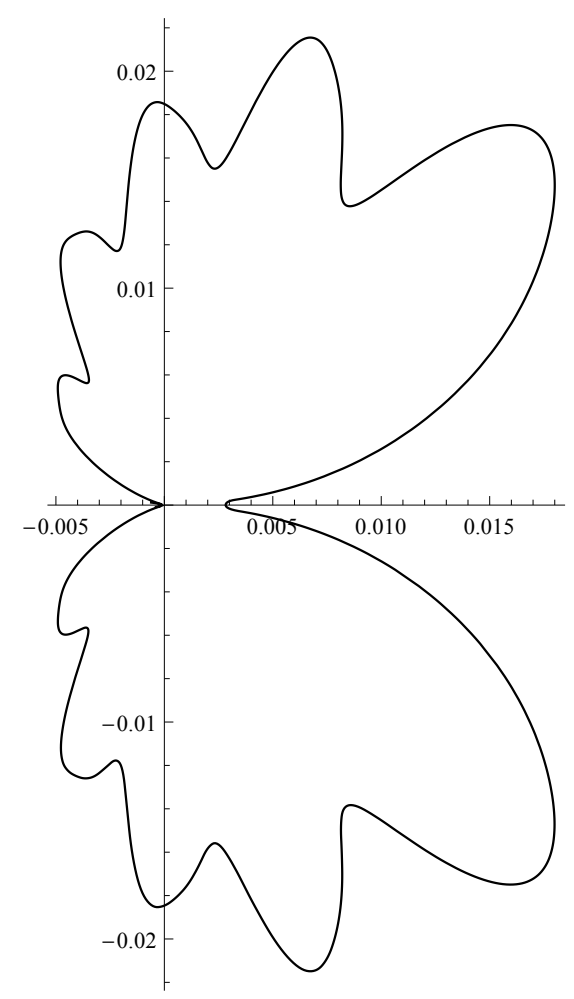

Figure 4.2: Far-field scattered pressure directivity for a NACA 0006 aerofoil, $k=8, \alpha_{i}=$ $0^{\circ}, M_{\infty}=0.6, \theta_{g}=0.01^{\circ}, k_{3}=0$, variables as defined for Chapter 2 .

which is formally the same order in $k$ and $r$ as the leading-edge field obtained in Chapter 2. The contribution from $\phi^{h}$ is negligible, since when $r$ is large, $\theta^{h} \rightarrow \pi / 2$ regardless of the size of $\theta$.

\subsubsection{Low-Frequency Solution}

Here we investigate the low-frequency limit, $k \ll 1$, of (4.2.0.5a). In this limit, the wavelength of the gust is much larger than the thickness of the ellipse, hence we would expect, to leading order, that the solution matches to that of the flat plate, i.e. is independent explicitly of the thickness parameter, $b$. The equivalent governing equation to (4.2.1.2) for the low-frequency solution is

$$
\bar{\phi}^{\prime \prime}=-r^{2} f(r, \theta)
$$

where $f(r, \theta)$ is given in (4.2.1.3), and the scalings, (4.2.1.4), still hold albeit $k$ is now small, hence $H_{1} \ll H_{0}$. The conditions (4.2.1.5) still hold, and we also still require zero normal velocity on the surface of the ellipse. We assume $r$ is $O(1)$ in deducing (4.2.2.1), thus we neglect terms of $O\left((k r)^{2}\right)$. Due to this, any far-field expansion we attempt to 
make of our solution $\left(r \gg k^{-1}\right)$ will be erroneous, thus we only use the low-frequency solution to provide us with details close to the boundary of the ellipse. This problem did not occur in the high-frequency case, because we were not required to place bounds on $r$ (beyond the fact that it is at least $O(1)$ close to the nose of the ellipse) in order to determine the governing equation, thus we could consider the far-field limit easily, and indeed extend our solution out of the small $-\theta$ region.

The particular solution to (4.2.2.1) satisfying (4.2.1.5) is

$$
\bar{\phi}^{p}=r^{2} \int_{\theta^{c}}^{\theta} d \theta^{\prime} \int_{\theta^{c}}^{\theta^{\prime}} f\left(r, \theta^{\prime \prime}\right) d \theta^{\prime \prime} .
$$

The homogeneous solution is

$$
\bar{\phi}^{h}=c(r)\left(\theta-\theta_{c}\right),
$$

where $c$ is an as yet undetermined function of $r$.

On the surface and under the small- $\theta$ approximation, $\boldsymbol{u}^{(I)} \cdot \boldsymbol{n}$ can be approximated by

$$
\frac{A_{1} r^{c}}{r}=O(1)
$$

and from (4.2.2.3) we know that

$$
\frac{\partial \bar{\phi}^{h}}{\partial n} \approx \frac{1}{1-b^{2}}\left[c^{\prime}(r)\left(1-b^{2}\right)-\frac{2 \theta c(r)}{1+b^{2}}\right]
$$

hence on the surface of the ellipse we require $c(r)=O\left(\left(1-b^{2}\right) \theta^{-1}\right)$. Note we do not solve explicitly for $\bar{\phi}^{h}$ in the low-frequency limit, as it is sufficient to know just the order of magnitude of the solution in order to deduce the effect of turbulence and to examine the leading-order turbulent pressure spectrum in this limit (we discuss this point in more detail shortly). We also have not calculated the normal derivative of the particular solution (which we would use to fully determine $c(r)$ ), as this is generated through scattering and hence is no greater in order of magnitude than the normal component of the gust at the boundary.

\subsection{Effect of Turbulence and the Turbulent Spectra}

We now consider the effect of homogeneous weak turbulence from upstream incident on the stagnation point of the ellipse (see Goldstein (1978b) and Hunt (1973) for early work on this topic). We assume the three dimensional upstream turbulent spectrum, $\Phi_{i, j}^{(\infty)}\left(k_{1}, k_{2}, k_{3}\right)$, is known. The one dimensional non-dimensionalised " $\nu, \mu$ " turbulent 
spectrum is defined by

$$
\Theta_{\nu, \mu}\left(\boldsymbol{x}, k_{1}\right)=\frac{1}{2 \pi} \int_{-\infty}^{\infty} R_{\nu, \mu}(\boldsymbol{x}, \tau) \mathrm{e}^{\mathrm{i} k_{1} \tau} d \tau,
$$

where $\nu, \mu=\{r, \theta, z\}$ and

$$
R_{\nu, \mu}(\boldsymbol{x}, \tau)=\overline{u_{\nu}(\boldsymbol{x}, t) u_{\mu}(\boldsymbol{x}, t+\tau)}
$$

is the one-point turbulent velocity correlation tensor, where the long over-bar denotes time averaging. $\Theta_{\nu, \mu}$ relates to the known upstream turbulent spectrum via

$$
\Theta_{\nu, \mu}\left(\boldsymbol{x}, k_{1}\right)=\int_{-\infty}^{\infty} \int_{-\infty}^{\infty} \bar{M}_{\nu, j} M_{\mu, n} \Phi_{j, n}^{(\infty)}(\boldsymbol{k}) d k_{2} d k_{3},
$$

where here the short over-bar denotes complex conjugation, and the $M_{\nu, j}$ are given by

$$
u_{\nu}=A_{j} M_{\nu, j}(r, \theta) \mathrm{e}^{\mathrm{i} k_{1}(\Delta-t)+\mathrm{i} k_{2} \Psi+\mathrm{i} k_{3} z} \quad \text { for } \quad \nu=r, \theta, z
$$

We assume the turbulence is isotropic and homogeneous upstream, so choose the von Kármán spectrum, as done by Goldstein (1978b),

$$
\Phi_{j, n}^{(\infty)}=\alpha \frac{\left(k_{1}^{2}+k_{2}^{2}+k_{3}^{2}\right) \delta_{j n}-k_{j} k_{n}}{\left(g_{2} / l^{2}+k_{1}^{2}+k_{2}^{2}+k_{3}^{2}\right)^{17 / 6}},
$$

where

$$
\alpha=\frac{55 g_{1} \overline{u_{\infty}^{2}}}{36 \pi l^{2 / 3}}
$$

the $g_{i}$ are constants to be determined from experimental data, and $l$ denotes the integral lengthscale of the turbulence. The far upstream magnitude of the vortical perturbation is given by $u_{\infty}$. This von Kármán spectrum is chosen because it agrees with the Kolmogoroff $-5 / 3$ law (Kolmogorov, 1941) which states that $E(k) \sim e^{2 / 3} k^{-5 / 3}$, where $E(k)=2 \pi \Phi_{i i}(k) k^{2}$ is the energy density (per unit mass) of the turbulence, and $e$ is the rate of energy dissipation per unit mass. Commonly this law is just quoted as $E(k) \sim k^{-5 / 3}$ as the dissipation is either unknown or not of interest. See Durbin \& Pettersson Reif (2001) for further details.

We define the one-dimensional turbulent pressure spectrum as

$$
\Theta_{p p}\left(\boldsymbol{x}, k_{1}\right)=\int_{-\infty}^{\infty} \int_{-\infty}^{\infty} \bar{N}_{i} N_{j} \Phi_{i, j}^{(\infty)}(\boldsymbol{k}) d k_{2} d k_{3}
$$


where the $N_{i}$ are defined by

$$
\frac{p}{\rho}=N_{j}(r, \theta) A_{j} \mathrm{e}^{\mathrm{i} k_{1}(\Delta-t)+\mathrm{i} k_{2} \Psi+\mathrm{i} k_{3} z},
$$

and the unsteady pressure can be found using

$$
p=-\rho_{0} \frac{D_{0} \phi}{D t}
$$

We present expressions for $\Theta_{p p}$ in Appendix B.

\subsubsection{High-Frequency Pressure Spectra in the Far Field}

We can extend our solution for $\Theta_{p p}$ into the far field, for $\theta=O(1)$, using (4.2.1.14). For the ellipse, in the far field, the homogeneous solution is negligible, hence $n_{i} \rightarrow 0$ for $i=2,3,4,5$ (where the $n_{i}$ are defined in Appendix B). Also $\phi^{p}=O\left(\frac{1}{k^{3 / 2} \sqrt{r}}\right)$, so $C=O\left(\frac{1}{\sqrt{r}}\right)$ (Appendix B). Substituting this into (B.1) gives

$$
\Theta_{p p} \sim \frac{2 g_{1} \overline{u_{\infty}^{2}} \sqrt{\pi}}{3 r} \frac{\Gamma\left(\frac{1}{3}\right)}{\Gamma\left(\frac{5}{6}\right)} \cos \theta \sin ^{4} \theta l^{2} M\left\{\begin{array}{ll}
g_{2}^{-4 / 3} & k_{1} l \ll 1 \\
\left(k_{1} l\right)^{-8 / 3} & k_{1} l \gg 1
\end{array} .\right.
$$

Hence for $k_{1} l \ll 1, \Theta_{p p}=O\left(l^{2} M r^{-1}\right)$ and for $k_{1} l \gg 1, \Theta_{p p}=O\left(k_{1}^{-8 / 3} l^{-2 / 3} M r^{-1}\right)$.

We construct a composite function to combine the high and low reduced frequency results for $\Theta_{p p}$ given by (4.3.1.1). This yields

$$
\Theta_{p p} \sim \frac{2 \overline{u_{\infty}^{2}} \sqrt{\pi} g_{1} l^{2} M \Gamma\left(\frac{1}{3}\right)}{3 r \Gamma\left(\frac{5}{6}\right)} \frac{g_{2}^{-4 / 3} \cos \theta \sin ^{4} \theta}{\left(1+\frac{\left(k_{1} l\right)^{8}}{g_{2}^{4}}\right)^{1 / 3}}=\frac{\text { const }}{r} h\left(\theta, k_{1} l\right) .
$$

We plot the functional behaviour of the far-field turbulent pressure spectrum in Figure 4.3, which we discuss later in Section 4.4.

\subsubsection{High- and Low-Frequency Surface Pressure Spectrum}

\section{Low-Frequency}

In the low-frequency regime, $k \ll 1$, the scattered pressure can be written as

$$
p=-\rho_{0} \mathrm{e}^{\mathrm{i} k_{1} t+\mathrm{i} k_{3} z}\left[-\mathrm{i} k_{1} \bar{\phi}+U_{r} \frac{\partial \bar{\phi}}{\partial r}+\frac{U_{\theta}}{r} \frac{\partial \bar{\phi}}{\partial \theta}\right],
$$


where $\bar{\phi}=\bar{\phi}^{h}+\bar{\phi}^{p}$. Evaluating each term in the small- $\theta$ approximation gives

$$
p^{p}=O\left(1-b^{2}\right), \quad p^{h}=O(1)
$$

where $p^{p, h}$ are the contributions to the pressure due to the particular and homogeneous solutions respectively. Thus, for $1-b \ll 1$, the homogeneous solution dominates the pressure on the surface of the ellipse near the nose. This agrees with the high-frequency limit, where the same result was found. This is a result of both high- and low-frequency theories because the particular solutions arise from source terms in the governing equations, which are generated by significant changes in momentum associated with the convected disturbance. It is sensible to assume that close to the boundary, in a thin wedge around the nose of the ellipse, the variation in momentum gradients is small, hence the volume sources make little contribution to the overall sound generated. The direct interaction between the boundary and the gust therefore generates the majority of the sound.

We write

$$
p \approx A_{1} N_{1} \mathrm{e}^{\mathrm{i} k_{1} t+\mathrm{i} k_{3} z}
$$

where $N_{1}$ is independent of $k_{2,3}$ and is $O(1)$ in the low-frequency regime. Hence using (4.3.0.7) we find that

$$
\Theta_{p p} \sim\left|N_{1}\right|^{2} \overline{u_{\infty}^{2}} g_{1} g_{2}^{-5 / 6}
$$

which is independent of $k_{1}$, and is $O(1)$ for $\theta,\left(1-b^{2}\right)=O\left(k^{1 / 2}\right)$ (these scalings of the small angle and thickness are consistent with the asymptotic analysis throughout when $k \ll 1$ ). Since the solution does not depend on the thickness of the ellipse at leading order then we are safe to indeed assume that the wavelength of the gust is so large that is does not see an elliptic body in the steady flow, but just a flat plate, and the pressure spectrum is dominated by vertical blocking of the gust velocity by the solid surface. To compute the coefficient $N_{1}$ we therefore look to Mish (2001), who considers spectra for a NACA 0015 aerofoil, based on the work by Amiet (1975) for a flat plate, and writes the pressure jump across a flat plate as

$$
\Delta p^{*}(x, y, t)=2 \pi \rho_{0}^{*} U^{*} b^{*} w_{0}^{*} g\left(x, k_{1}, k_{3}\right) \mathrm{e}^{\mathrm{i} k_{3}^{*} z^{*}-k_{1}^{*} U^{*} t^{*}}
$$

in dimensional form. Here $g$ is the transfer function between turbulent velocity and aerofoil pressure jump, and $w_{0}^{*}$ is the magnitude of the gust velocity. We can therefore 
write that the cross power spectral density at a given point is

$$
S_{q q}\left(x, x, 0, \omega^{*}\right)=4\left(2 \pi \rho_{0}^{*} b^{*}\right)^{2} \int_{-\infty}^{\infty} \int_{-\infty}^{\infty}\left|g\left(x,-\omega / U, k_{3}\right)\right|^{2} \Phi_{1,1}^{(\infty)}\left(-\omega^{*} / U^{*}, k_{2}^{*}, k_{3}^{*}\right) d k_{2}^{*} d k_{3}^{*},
$$

where $\omega^{*}$ is the circular frequency, $U^{*}$ is the steady free-stream velocity, and $b^{*}$ is the semi-chord length of the flat plate. Mish (2001) provides us with the transfer function for a thin aerofoil, which, in our limit of small $k_{1}^{*}$ and low Mach number, is

$$
g\left(x, k_{1}, k_{3}\right)=-\left(1-\sqrt{\frac{x}{2}}\left(1-\operatorname{erf}\left[\sqrt{2(2-x) k_{3}}\right]\right)\right) \frac{\mathrm{e}^{-\mathrm{i} k_{3} x}}{\pi^{3 / 2} \sqrt{x} \sqrt{k_{3}+\mathrm{i} k_{1}}} .
$$

Equation (4.3.2.6) can then be evaluated numerically. We see that (4.3.2.7) is dependent on $k_{1}$ thus we would naively expect our expression given in (4.3.2.4) to be incorrect, and $\Theta_{p p}$ should be dependent on $k_{1}$ in the low-frequency limit. However, we must now recall that whilst the overall scaling on our asymptotic estimate of $\Theta_{p p}$ is independent explicitly of $k_{1}$, this only arises through cancellation of orders of magnitude of $k_{1}$ with the thickness factor $\left(1-b^{2}\right)$, thus to compute $N_{1}$ to agree with experimental data, we must evaluate (4.3.2.6) for the value of small $k_{1}$ associated to the thickness of the aerofoil in the experiment. This then gives us a low-frequency asymptotic estimate for the turbulent pressure spectrum.

We evaluate (4.3.2.6) close to the nose of the aerofoil, at $x=0.01$ say, and for a circular frequency of 2.31, steady free-stream velocity of 30, semi-chord length of 0.3 , and integral lengthscale of turbulence (measured close to the surface) 0.0078 , to find

$$
S_{q q} \approx 2.06
$$

We choose the position $x$, integral lengthscale, $l$, and free-stream velocity $U$ in this way to agree with the experimental set up of Mish \& Devenport (2003). The circular frequency is chosen to concur with our relation between thickness of the aerofoil and frequency of the gust (which in the low-frequency limit is $\left(1-b^{2}\right)=O\left(k^{1 / 2}\right)$ ). We do not consider a high reduced frequency limit in this case because it would be unphysical for turbulence to have such a large integral lengthscale to allow $k_{1} \ll 1$ but $k_{1} l \gg 1$.

\section{High-Frequency}

For small $\theta$ and high frequency, $k_{1} \gg 1$, we consider the turbulent spectrum on the nose of the ellipse by considering the limiting behaviour of (B.1) in Appendix B. It is expected that this depends on the exact location of the stagnation point, and hence 


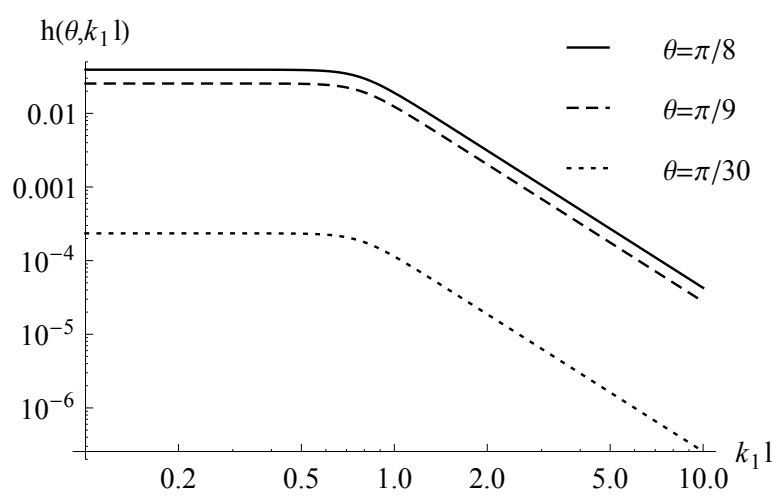

Figure 4.3: Plot of $h\left(\theta, k_{1} l\right)$ governing the turbulent pressure spectrum far from the ellipse, for varying values of $\theta$. The constant, $g_{2}$ is chosen to be 0.6 .

depends explicitly on the thickness parameter $b$, unlike in the far field where the solution decays exponentially and this dependence is negligible (as previously seen in the highfrequency limit in the far field). At intermediate distances, solutions for both small and $O(1)$ values of $\theta$ depend on thickness for the high- and low-frequency limits. Indeed from Appendix B we find in the high-frequency limit,

$$
\Theta_{p p} \sim \frac{g_{1} \overline{u_{\infty}^{2}} \sqrt{\pi} \Gamma\left(\frac{1}{3}\right) l^{2} M}{3 \Gamma\left(\frac{5}{6}\right)\left(1-b^{2}\right)^{2}} \begin{cases}g_{2}^{-4 / 3} & k_{1} l \ll 1 \\ \left(k_{1} l\right)^{-8 / 3} & k_{1} l \gg 1\end{cases}
$$

so $\Theta_{p p}=O\left(k_{1} l^{2} M\right)$ for $k_{1} l \ll 1$ and $=O\left(k_{1}^{-5 / 3} l^{-2 / 3} M\right)$ for $k_{1} l \gg 1$ when we take account of the scaling of the thickness. A composite function can be constructed similarly for the far-field high-frequency solution.

\subsection{Results}

We first compare our high-frequency far-field solutions found in Section 4.3.1 to those obtained, also asymptotically, by Durbin (1978). From Figure 4.3 we see that as $\theta$ decreases, so too does $h\left(\theta, k_{1} l\right)$ for all $k_{1} l$, whilst maintaining the overall shape of the curve. This can be anticipated through the results obtained by Durbin (1978); there he analysed the turbulent pressure spectrum for uniform flow at zero angle of attack around a circular cylinder, and found that at the stagnation point and along the zero-streamline, the pressure spectrum decays exponentially fast for high frequencies. This is due to the piling up of eddies at the stagnation point that cancel each other out. It is sensible to assume a similar effect takes place for our problem.

On the solid surface for small $\theta$ the high-frequency limit of the turbulent pressure 


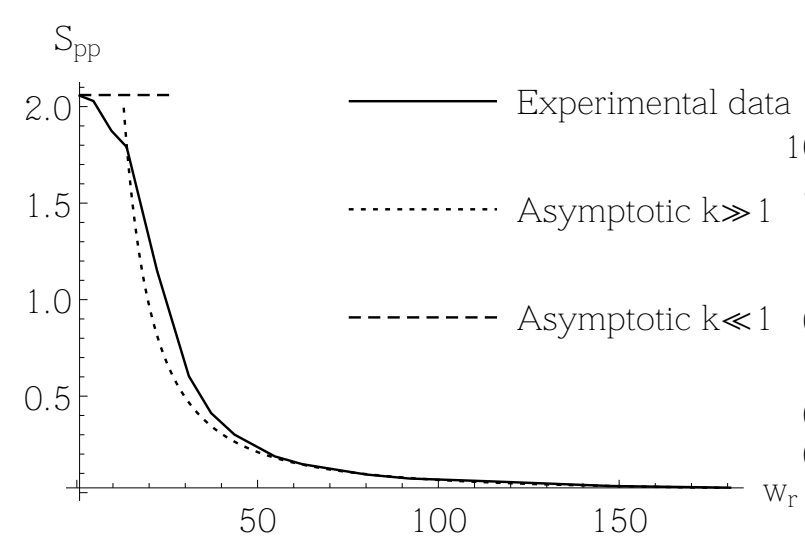

(a) On a linear scale.

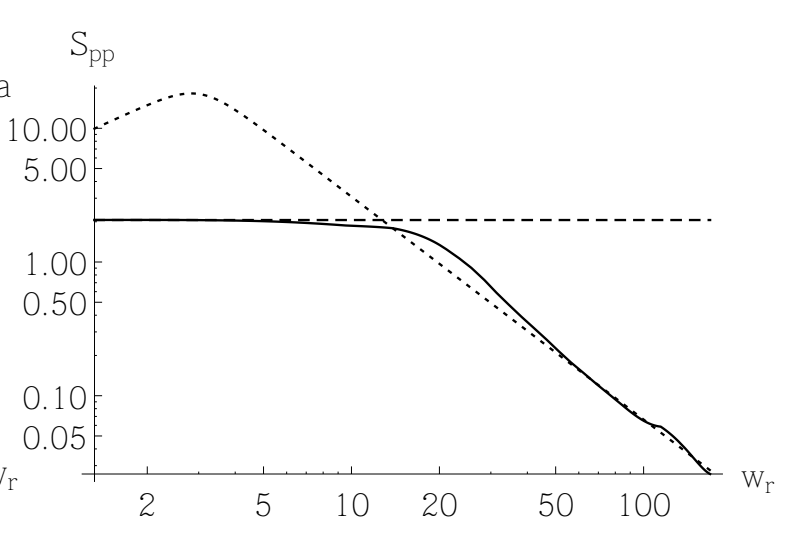

(b) On a log-log scale.

Figure 4.4: Asymptotically obtained cross-PSD (normalised by $4 \pi U$ ) close to $\theta=0$ with $U=30, b=2, \rho_{0}=1, l=0.0078$, and $M=0.09$, compared to experimental data, on the surface of a NACA 0015 aerofoil. The horizontal axis measures frequency, and the vertical axis measures the surface pressure spectrum. Experimental results are courtesy of William Devenport (pers. comm.).

spectrum is given by (4.3.2.9), and the low-frequency limit of the surface turbulent pressure spectrum is given by (4.3.2.8). It can be seen from (4.3.2.6) that the crossPSD is directly related to the turbulent pressure spectrum, thus we can compare our asymptotic results to experimental readings for PSD, and related quantities. We compare our asymptotic solutions for high- and low-frequency on the surface of the ellipse (Section 4.3.2) against the experimental data obtained by Mish \& Devenport (2003) in Figure 4.4. It is clear that the asymptotic results are a good match to the experimental data in both the high- and low-frequency domains, therefore the new solution for the acoustic potential close to the stagnation point found in this chapter reveals a turbulent pressure spectra that agrees well with experimental data. We therefore expect that this acoustic solution gives a physically sensible unsteady pressure at the leading edge of an aerofoil, i.e. one with no singularity. We also note that, whilst the potential solution close to the body scales with an inverse power of $r$ (see Section 4.2), this is not singular since the origin is at the centre of the body and not at the leading edge itself.

The far-field limit of the high-frequency solution, given in Section 4.3.1, along with the sound pressure levels measured experimentally by Geyer et al. (2012) are plotted in Figure 4.5. We see a good agreement for large $k_{1} l$, however in the low-frequency region the asymptotic approximation no longer matches the experimental results. This is because the integral lengthscale of turbulence is fixed in an experiment, however our highfrequency approximation has allowed for us to vary $l$ so that $k_{1} l$ can be very small whilst 


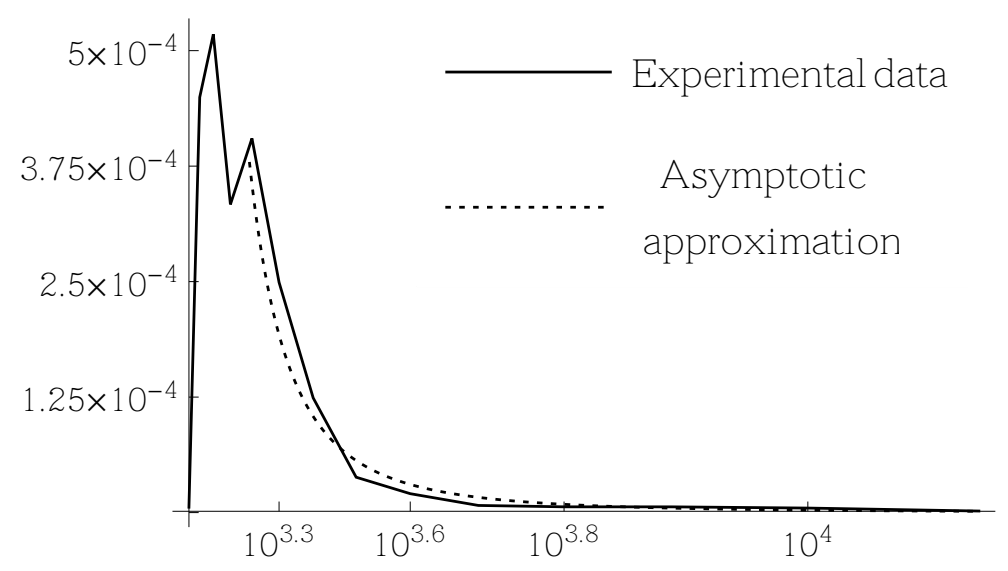

Figure 4.5: Sound pressure levels in the far field from the leading edge of an SD7003 aerofoil measured experimentally, compared to the asymptotic result for $k \gg 1$. The horizontal axis measures frequency and the vertical axis measures the pressure level. Experimental data comes courtesy of Thomas Geyer (pers. comm.). In the asymptotic result we have set $l=0.01, U=35, M=0.09, g_{1}=0.2$ and $g_{2}=0.56$.

$k_{1}$ is still very large. For us to obtain a matching in the high-frequency limit, we would require an incredibly small integral lengthscale of turbulence to be used experimentally which is not usually considered as it is not physically relevant to the effects of real turbulence on aerofoils.

\subsection{Conclusions}

We have found approximations for the turbulent pressure spectra generated by homogeneous isotropic turbulence far upstream of a thin elliptic cylinder in a background uniform steady flow. Both high- and low-frequency turbulence has been considered. In regions close to the leading-edge stagnation point, and in the far field at angles close to the zero-streamline, both approximations show good agreement with current experimental data. Specific bounds have been placed on the thickness of the ellipse, the small angles defining how close to the stagnation streamline we must be, and on the Mach number of the steady flow. These bounds are given as powers of the frequency of the incident turbulence, $k$, in order to allow asymptotic series to be truncated for algebraic simplicity. As mentioned in Chapter 3, despite this work only being valid for low Mach number flows, we believe that these results are still of great importance given the complexity of the problem and the difficulty current computational schemes face in tackling the high-frequency regime. 
The high-frequency velocity potential found in the far-field for $O(1)$ values of $\theta$, (4.2.1.14), shows that as we move out of our designated zero-streamline region, we obtain a matching form to the leading-edge inner velocity potential found in Chapter 2. This concludes that it should be possible to construct a further inner solution, for $O\left(k^{-1}\right)$ values of $\theta$, to the one obtained in Chapter 2 to properly assess the effects of highfrequency gust-aerofoil interaction.

We have seen that at large distances upstream from the leading edge, the radiated power approximation is in agreement with current experimental data by Geyer et al. (2012). We must note that other experimental data has been collected using a single microphone, but the asymptotics in this chapter require certain restrictions on the angle with respect to the leading edge. A microphone positioned outside the region of validity of these results will give us nothing worth comparing against, and this restriction limits the amount of available experimental data. We have also seen that our asymptotic results close to the nose of the body are in good agreement with experimental data from Mish \& Devenport (2003).

Crucially, in this chapter we have constructed a solution for the pressure generated on the surface of a thick body whose nose has a radius of curvature of order thickness squared that is not singular near the nose itself, and whose solution depends on the frequency of the incident turbulence, and the thickness of the body. 


\section{List of Symbols for Chapter 4}

$a_{i} \quad$ constants in solution to steady flow drift function, velocity, and potential.

$\boldsymbol{A}$ amplitude of incident gust, $=\left(A_{1}, A_{2}, A_{3}\right)$ in Cartesian coordinates.

$b \quad$ Joukowski transformation parameter, $\tau=\left(1-b^{2}\right) /\left(1+b^{2}\right)$.

c speed of sound, $c^{2}=M^{-2}+c_{1}^{2}$.

$f \quad$ modified form of the acoustic source term.

$g_{ \pm} \quad$ phase functions in integrand for $\bar{\phi}^{p}$.

$G \quad$ Green's function for the velocity potential.

$h \quad$ composite function determining the behaviour of the high-frequency turbulent pressure spectra in the far field as a function of reduced frequency.

$H_{i} \quad$ amplitude functions in the source of the acoustic equation.

$\boldsymbol{k} \quad$ wavevector of a single incident gust mode, $=k\left(k_{1}^{\dagger}, k_{2}^{\dagger}, k_{3}^{\dagger}\right)$ in Cartesian coordinates.

$l \quad$ integral lengthscale of turbulence.

$M \quad$ Mach number of the steady flow.

$M_{\nu, j}$ amplitude of the $\nu$-component of velocity for a single gust mode with wavevector $\boldsymbol{k}$, dependent on $A_{j}$.

$N_{i} \quad$ amplitude of the pressure of a single gust mode dependent on $A_{i}$.

$p$ pressure.

$R \quad$ function arising in the high-frequency $\bar{\phi}^{h}$ solution.

$R_{\nu, \mu}$ one-point turbulent velocity correlation tensor.

$\boldsymbol{u}_{\infty} \quad$ velocity of a single incident gust mode, $=\boldsymbol{u}^{(I)}+\nabla \phi$.

$\boldsymbol{u}^{(I)}$ evolution of the unsteady incident gust.

$\boldsymbol{U} \quad$ steady flow $=\boldsymbol{U}_{0}+M^{2} \boldsymbol{U}_{1}$, and far upstream equals $\boldsymbol{U}_{\infty}=U_{\infty} \boldsymbol{e}_{x}$.

$w \quad k_{1}^{\dagger 2} M^{2}-k_{3}^{\dagger 2}$.

$z \quad$ complex coordinate for elliptic geometry, $=r e^{i \theta}$.

$\alpha \quad$ constant in the upstream pressure spectrum, proportional to $g_{1}$.

$\Delta \quad$ drift function. 
$\delta \quad$ streamline close to the zero-streamline.

$\epsilon \quad$ upper limit of validity of the small $\theta$ approximation.

$\rho \quad$ density, $=\rho_{0}+M^{2} \rho_{1}$.

$\varrho \quad$ radial coordinate in pre-transformed space for circular cylinder.

$\Phi \quad$ velocity potential for the steady flow, $=\Phi_{0}+M^{2} \Phi_{1}$.

$\phi \quad$ velocity potential for the acoustics generated by the gust, $=\phi_{0}+M^{2} \phi_{1}$.

$\varphi \quad$ angular coordinate in pre-transformed space for circular cylinder.

$\bar{\phi} \quad \phi \mathrm{e}^{\mathrm{i} k_{1} t-\mathrm{i} k_{3} z}$, and $\bar{\phi}=\bar{\phi}^{h}+\bar{\phi}^{p}$ is a sum of homogeneous and particular solutions.

$\Phi_{i, j}^{(\infty)}$ three-dimensional upstream turbulent pressure spectrum.

$\Psi \quad$ streamfunction for the steady flow, $=\Psi_{0}+M^{2} \Psi_{1}$.

$\tau \quad$ small thickness parameter.

$\Theta_{\nu, \mu}$ one-dimensional turbulent velocity spectrum.

$\theta^{c} \quad$ angle at which the $\delta$-streamline begins to curve on approach to the ellipse.

$\Theta_{p p} \quad$ turbulent pressure spectrum.

$\theta_{s}^{\prime} \quad$ point of stationary phase of $\bar{\phi}^{p},=\theta_{s}^{0}+M^{2} \theta_{s}^{1}$.

$\omega \quad$ frequency of the Green's function, $G,=k \Omega$. 


\section{Appendix A}

Here we evaluate the drift function, $\Delta$, needed in Section 4.1. Using (4.1.3.4) we can write the velocity component $u_{\theta}$ as

$$
\begin{aligned}
u_{\theta}= & a_{1} \sin \theta+\delta\left(a_{2}+a_{3} f(\theta)+a_{4} \cos 2 \theta\right) \frac{1}{f(\theta)}+\delta^{2} \frac{\csc \theta}{f(\theta)}\left(a_{5} f(\theta)+a_{6}+a_{7} \sin ^{2} \theta\right) \\
& +\delta M^{2}\left(a_{8}+a_{9} f(\theta)+a_{10} \cos 2 \theta+a_{11} f(\theta) \cos 2 \theta+a_{12} \cos 4 \theta\right) \frac{1}{f(\theta)} \\
& +M^{2}\left(a_{13} \sin \theta+a_{14} \sin 3 \theta\right)
\end{aligned}
$$

where

$$
f(\theta)=\sqrt{\left(1-b^{2}\right)^{2}-\left(1+b^{2}\right)^{2} \sin ^{2} \theta}
$$

and $\theta$ measures from the positive $x$-axis anticlockwise in the standard manner. For $\theta \in[\pi-2 \arctan [(1-b) /(1+b)], \pi], f(\theta)>0$ hence the square root function is well defined. We therefore must choose our limit of "small $\theta$ " to be below

$$
\pi-2 \arctan [(1-b) /(1+b)]
$$

so that $f(\theta)$ is real. This upper limit clearly decreases as the thickness of the ellipse decreases. We still work with a general value of $b$ (governing the thickness of the ellipse) but bear in mind there is an upper limit of $\theta$ for any further series expansion.

The constants $a_{i}$ are given by

$$
\begin{aligned}
& a_{1}=-\frac{2\left(1+b^{2}\right)}{\left(1-b^{2}\right)^{2}}, \\
& a_{2}=-\frac{2\left(b^{2}-6 b^{4}+b^{6}\right)}{\left(1+b^{2}\right)\left(1-b^{2}\right)^{3}}, \\
& a_{3}=\frac{\left(b^{4}+6 b^{2}+1\right)}{\left(1+b^{2}\right)\left(1-b^{2}\right)^{2}}, \\
& a_{4}=-\frac{2 b^{2}\left(1+b^{2}\right)}{\left(1-b^{2}\right)^{2}} \\
& a_{5}=-\frac{1+3 b^{8}+32 b^{6}+26 b^{2}+57 b^{4}}{4\left(1-b^{2}\right)^{2}\left(1+b^{2}\right)^{3}}, \\
& a_{6}=\frac{b^{2}\left(6+14 b^{2}+7 b^{4}\right)}{\left(1-b^{2}\right)\left(1+b^{2}\right)^{3}}, \\
& a_{7}=-\frac{b^{2}\left(6+22 b^{2}+7 b^{4}\right)}{\left(1-b^{2}\right)^{3}\left(1+b^{2}\right)}, \\
& a_{8}=-\frac{b^{2}\left(-7+76 b^{2}+331 b^{4}+412 b^{6}+75 b^{8}-24 b^{10}+b^{12}\right)}{3\left(1-b^{2}\right)^{7}\left(1+b^{2}\right)},
\end{aligned}
$$




$$
\begin{aligned}
& a_{9}=-\frac{63+309 b^{2}+299 b^{4}-303 b^{6}-55 b^{8}+187 b^{10}+9 b^{12}-5 b^{14}}{12\left(1-b^{2}\right)^{7}\left(1+b^{2}\right)}, \\
& a_{10}=\frac{b^{2}\left(1+b^{2}\right)\left(-1+238 b^{2}+392 b^{4}-54 b^{6}+b^{8}\right)}{6\left(1-b^{2}\right)^{7}}, \\
& a_{11}=\frac{\left(1+b^{2}\right)\left(11+12 b^{2}+30 b^{3}-12 b^{4}+24 b^{5}+4 b^{6}-6 b^{7}+b^{8}\right)}{2\left(1-b^{2}\right)^{6}}, \\
& a_{12}=\frac{b^{2}\left(1+b^{2}\right)^{3}\left(b^{4}-8 b^{2}-5\right)}{2\left(1-b^{2}\right)^{7}} \\
& a_{13}=\frac{2\left(1+b^{2}\right)\left(2+b^{2}\right)\left(1+2 b^{2}\right)}{3\left(1-b^{2}\right)^{4}} \\
& a_{14}=-\frac{\left(1+b^{2}\right)^{3}}{2\left(1-b^{2}\right)^{4}}
\end{aligned}
$$

Using (4.1.3.4) we obtain

$$
\begin{aligned}
\frac{r}{u_{\theta}} \sim & \frac{\left(1+b^{2}\right)}{a_{1}} \csc \theta-M^{2} \frac{\left(1+b^{2}\right)}{a_{1}^{2}}\left(a_{13}+a_{14} \csc \theta \sin 3 \theta\right) \csc \theta \\
& +\delta \csc ^{2} \theta \frac{1}{a_{1}^{2}}\left[\frac{a_{1}\left(1-b^{2}\right)^{2}}{2\left(1+b^{2}\right)}-\frac{1+b^{2}}{f(\theta)}\left(a_{2}+a_{4} \cos 2 \theta+a_{3} f(\theta)\right)\right] \\
& +\delta M^{2} \csc ^{2} \theta \frac{1}{a_{1}^{3}}\left\{-2\left(a_{13}+a_{14}+2 a_{14} \cos 2 \theta\right)\left(\frac{a_{1}\left(1-b^{2}\right)^{2}}{2\left(1+b^{2}\right)}\right.\right. \\
& \left.-\frac{1+b^{2}}{f(\theta)}\left(a_{2}+a_{4} \cos 2 \theta+a_{3} f(\theta)\right)\right)+a_{1}\left[\frac{\left(1-b^{2}\right)^{2}}{2\left(1+b^{2}\right)}\left(a_{13}+a_{14}+2 a_{14} \cos 2 \theta\right)\right. \\
& -\frac{\left(1+b^{2}\right)}{f(\theta)}\left(a_{8}+a_{10} \cos 2 \theta+a_{12} \cos 4 \theta+\left(a_{9}+a_{11} \cos 2 \theta\right) f(\theta)\right) \\
& +a_{1} \sin \theta\left(\frac{1}{2}\left(1+b^{2}\right) \sin \theta-\frac{\left(1-b^{2}\right)^{2} \csc \theta}{\left.\left.24\left(1+b^{2}\right)\right]\right\}}\right. \\
& +\delta^{2} \csc ^{2} \theta \frac{1}{8 a_{1}^{2}\left(1+b^{2}\right)^{3}}\left[a_{1}\left(1-b^{2}\right)^{2}-\frac{4\left(1-b^{4}\right)^{2}}{f(\theta)}\left(a_{2}+a_{4} \cos 2 \theta+a_{3} f(\theta)\right)\right. \\
& +\frac{8\left(1+b^{2}\right)^{4}}{a_{1} f(\theta)^{2}}\left(-a_{1} a_{6} f(\theta)-a_{1} a_{5} f(\theta)^{2}-a_{1} a_{7} f(\theta) \sin ^{2} \theta\right. \\
+ & {\left.\left.\left[a_{2}+a_{4} \cos 2 \theta+a_{3} f(\theta)\right]^{2}\right)\right]+O\left(M^{4}, \delta^{3}, \delta^{2} M^{2}\right) . }
\end{aligned}
$$

Integrating (A.3) to obtain (4.1.3.2) requires:

$$
\begin{aligned}
& I_{1}=\int \csc \theta d \theta=\log [\tan (\theta / 2)] \\
& I_{2}=\int \csc ^{2} \theta d \theta=-\cot \theta
\end{aligned}
$$




$$
\begin{aligned}
& I_{3}=\int \csc ^{2} \theta \sin 3 \theta d \theta=4 \cos \theta+3 \log \left[\tan \frac{\theta}{2}\right] \\
& I_{4}=\int \frac{\csc ^{2} \theta}{f(\theta)} d \theta=\frac{1}{\sqrt{2}\left(1-b^{2}\right)^{2} g(\theta)}\left\{\left(b^{2}+1\right)^{2} \sin 2 \theta-2\left(1-b^{2}\right)^{2} \cot \theta\right. \\
& \left.+\sqrt{2}\left(1-b^{2}\right) g(\theta) F\left(\theta \mid \frac{\left(b^{2}+1\right)^{2}}{\left(1-b^{2}\right)^{2}}\right)-\sqrt{2}\left(1-b^{2}\right) g(\theta) E\left(\theta \mid \frac{\left(b^{2}+1\right)^{2}}{\left(1-b^{2}\right)^{2}}\right)\right\}, \\
& I_{5}=\int \frac{\csc ^{2} \theta}{f(\theta)} \cos 2 \theta d \theta= \\
& \frac{g(\theta) \cot \theta}{\sqrt{2}\left(1-b^{2}\right)^{2}}-\frac{1}{\left(1-b^{2}\right)}\left[F\left(\theta \mid \frac{\left(b^{2}+1\right)^{2}}{\left(1-b^{2}\right)^{2}}\right)+E\left(\theta \mid \frac{\left(b^{2}+1\right)^{2}}{\left(1-b^{2}\right)^{2}}\right)\right], \\
& I_{6}=\int \csc ^{2} \theta \cos 2 \theta d \theta=-2 \theta-\cot \theta \text {, } \\
& I_{7}=\int \frac{\csc ^{2} \theta}{f(\theta)} \cos ^{2} 2 \theta d \theta=\frac{1}{\left(1-b^{2}\right)\left(b^{2}+1\right)^{2}}\left[\left(b^{4}-14 b^{2}+1\right) F\left(\theta \mid \frac{\left(b^{2}+1\right)^{2}}{\left(1-b^{2}\right)^{2}}\right)\right. \\
& \left.-\left(5 b^{4}-6 b^{2}+5\right) E\left(\theta \mid \frac{\left(b^{2}+1\right)^{2}}{\left(1-b^{2}\right)^{2}}\right)\right]-\frac{g(\theta) \cot \theta}{\sqrt{2}\left(1-b^{2}\right)^{2}}, \\
& I_{8}=\int \frac{\csc ^{2} \theta}{f(\theta)} \cos 4 \theta d \theta=\frac{1}{\left(1-b^{2}\right)\left(b^{2}+1\right)^{2}}\left[\left(b^{4}-30 b^{2}+1\right) F\left(\theta \mid \frac{\left(b^{2}+1\right)^{2}}{\left(1-b^{2}\right)^{2}}\right)\right. \\
& \left.-\left(9 b^{4}-14 b^{2}+9\right) E\left(\theta \mid \frac{\left(b^{2}+1\right)^{2}}{\left(1-b^{2}\right)^{2}}\right)\right]-\frac{g(\theta) \cot \theta}{\sqrt{2}\left(1-b^{2}\right)^{2}} \\
& I_{9}=\int \frac{1}{f(\theta)} d \theta=\frac{F\left(\theta \mid \frac{\left(b^{2}+1\right)^{2}}{\left(b^{2}-1\right)^{2}}\right)}{1-b^{2}} \\
& I_{10}=\int \frac{\csc ^{2} \theta}{f(\theta)^{2}} d \theta=\frac{\left(b^{2}+1\right)^{2} \tanh ^{-1}\left(\frac{2 b \tan \theta}{1-b^{2}}\right)-2 b\left(1-b^{2}\right) \cot \theta}{2 b\left(1-b^{2}\right)^{3}}, \\
& I_{11}=\int \frac{\csc ^{2} \theta}{f(\theta)^{2}} \cos ^{2} 2 \theta d \theta= \\
& \frac{1}{2\left(1-b^{2}\right)^{3}}\left(\frac{\left(b^{4}-6 b^{2}+1\right)^{2} \tanh ^{-1}\left(\frac{2 b \tan \theta}{1-b^{2}}\right)}{b\left(b^{2}+1\right)^{2}}-2\left(1-b^{2}\right) \cot \theta\right)-\frac{4 \theta}{\left(b^{2}+1\right)^{2}}, \\
& I_{12}=\int \frac{\csc ^{2} \theta}{f(\theta)^{2}} \cos 2 \theta d \theta=-\frac{2\left(1-b^{2}\right) \cot (\theta)+\left(b^{3}-6 b+\frac{1}{b}\right) \tanh ^{-1}\left(\frac{2 b \tan (\theta)}{1-b^{2}}\right)}{2\left(1-b^{2}\right)^{3}},
\end{aligned}
$$


where

$$
g(\theta)=\sqrt{b^{4}+\left(b^{2}+1\right)^{2} \cos 2 \theta-6 b^{2}+1}
$$

For $\theta$ sufficiently close to $\pi$ the quantity within the square root defined in $g(\theta)$ is positive, hence $g$ is real. This boundary is $\pi-\frac{1}{2} \cos ^{-1}\left[\frac{-b^{4}+6 b^{2}-1}{\left(1+b^{2}\right)^{2}}\right]$, which for $b$ close to 1 is $\sim \pi-(1-b)$, hence is closer to $\pi$ than the limit of real $f(\theta)$. We therefore take this as the new limit of validity for our expansions. In the above expression, $F(x \mid m)$ and $E(x \mid m)$ denote the elliptic integrals of the first and second kind respectively (Abramowitz \& Stegun, 1964, p. 589).

We now redefine our coordinates to measure $\theta$ clockwise from the zero-streamline. The origin remains at the centre of the ellipse (see Figure 4.1). We expand the integrals, (A.4), for small $\theta$ now, to obtain the expressions near the frontal stagnation point. These expansions are

$$
\begin{aligned}
I_{1} & \sim \log \left[\frac{\theta}{2}\right]+\frac{\theta^{2}}{12}+O\left(\theta^{3}\right) \\
I_{2} & \sim-\frac{1}{\theta}+\frac{\theta}{3}+O\left(\theta^{3}\right) \\
I_{3} & \sim 3 \log \left[\frac{\theta}{2}\right]+4-\frac{7 \theta^{2}}{4}+O\left(\theta^{3}\right), \\
I_{4} & \sim-\frac{1}{\left(1-b^{2}\right) \theta}+\frac{\left(5 b^{4}+2 b^{2}+5\right) \theta}{6\left(1-b^{2}\right)^{3}}+O\left(\theta^{3}\right), \\
I_{5} & \sim-\frac{1}{\left(1-b^{2}\right) \theta}-\frac{\left(7 b^{4}-26 b^{2}+7\right) \theta}{6\left(1-b^{2}\right)^{3}}+O\left(\theta^{3}\right), \\
I_{6} & \sim-\frac{1}{\theta}-\frac{5 \theta}{3}+O\left(\theta^{3}\right), \\
I_{7} & \sim-\frac{1}{\left(1-b^{2}\right) \theta}-\frac{\left(19 b^{4}-50 b^{2}+19\right) \theta}{6\left(1-b^{2}\right)^{3}}+O\left(\theta^{3}\right), \\
I_{8} & \sim \frac{1}{\left(b^{2}-1\right) \theta}+\frac{\left(43 b^{4}-98 b^{2}+43\right) \theta}{6\left(b^{2}-1\right)^{3}}+O\left(\theta^{3}\right), \\
I_{9} & \sim \frac{\theta}{1-b^{2}}+O\left(\theta^{3}\right), \\
I_{10} & \sim-\frac{1}{\left(1-b^{2}\right)^{2} \theta}+\frac{4\left(b^{4}+b^{2}+1\right) \theta}{3\left(1-b^{2}\right)^{4}}+O\left(\theta^{3}\right), \\
I_{11} & \sim-\frac{1}{\left(1-b^{2}\right)^{2} \theta}-\frac{4\left(2 b^{4}-7 b^{2}+2\right) \theta}{3\left(1-b^{2}\right)^{4}}+O\left(\theta^{3}\right), \\
I_{12} & \sim-\frac{1}{\left(1-b^{2}\right)^{2} \theta}-\frac{2\left(b^{4}-8 b^{2}+1\right) \theta}{3\left(1-b^{2}\right)^{4}}+O\left(\theta^{3}\right) .
\end{aligned}
$$


We therefore obtain the drift function (non-dimensionalised with respect to $L^{*-1} U^{*}$ ) for uniform flow around a thin ellipse;

$$
\begin{aligned}
\Delta(\theta) \approx & \text { const. }-r \cos \theta+\frac{\left(1+b^{2}\right)}{a_{1}} I_{1}-M^{2} \frac{\left(1+b^{2}\right)}{a_{1}^{2}}\left(a_{13} I_{1}+a_{14} I_{3}\right) \\
& +\frac{\delta}{a_{1}^{2}}\left(a_{1} \frac{\left(1-b^{2}\right)^{2}}{\left(1+b^{2}\right)} I_{2}-\left(1+b^{2}\right)\left[a_{2} I_{4}+a_{4} I_{5}+a_{3} I_{2}\right]\right) \\
& +\frac{\delta^{2}}{8 a_{1}^{2}\left(1+b^{2}\right)^{3}}\left\{a_{1}\left(1-b^{2}\right)^{2} I_{2}-4\left(1-b^{4}\right)^{2}\left[a_{2} I_{4}+a_{4} I_{5}+a_{3} I_{2}\right]\right. \\
& +\frac{8\left(1+b^{2}\right)^{4}}{a_{1}}\left(-a_{1} a_{6} I_{4}-a_{1} a_{5} I_{2}-a_{1} a_{7} I_{9}+a_{2}^{2} I_{10}+a_{4} I_{11}+a_{3}^{2} I_{2}+2 a_{2} a_{4} I_{12}\right. \\
& \left.\left.+2 a_{2} a_{3} I_{4}+2 a_{4} a_{3} I_{5}\right)\right\}+\frac{\delta M^{2}}{a_{1}^{3}}\left\{\frac{-a_{1}\left(1-b^{2}\right)^{2}}{\left(1+b^{2}\right)}\left[\left(a_{13}+a_{14}\right) I_{2}+2 a_{14} I_{6}\right]\right. \\
& +2\left(1+b^{2}\right)\left[\left(a_{13}+a_{14}\right)\left(a_{2} I_{4}+a_{4} I_{5}+a_{3} I_{2}\right)+2 a_{14}\left(a_{2} I_{5}+a_{4} I_{7}+a_{3} I_{6}\right)\right] \\
& +a_{1}\left(\frac{\left(1-b^{2}\right)^{2}}{2\left(1+b^{2}\right)}\left[\left(a_{13}+a_{14}\right) I_{2}+2 a_{14} I_{6}\right]-\left(1+b^{2}\right)\left(a_{8} I_{4}+a_{10} I_{5}+a_{12} I_{8}\right.\right. \\
& \left.\left.\left.+a_{9} I_{2}+a_{11} I_{6}\right)+a_{1}\left[\frac{1}{2}\left(1+b^{2}\right) \theta-\frac{\left(1-b^{2}\right)^{2}}{24\left(1+b^{4}\right)} I_{2}\right]\right)\right\}
\end{aligned}
$$

where the constant depends on $r^{c}, \theta^{c}$ and $\delta$. Recall $\left(r^{c}, \theta^{c}\right)$ denotes the position at which the $\delta$-streamline begins to veer away from the uniform zero-streamline as illustrated in Figure 4.1. These points approximate to

$$
\begin{aligned}
\theta^{c}= & \frac{\delta}{r^{c}}, \\
r^{c}= & \frac{\left(1+b^{2}\right)^{4}}{1+6 b^{2}+8 b^{4}+2 b^{6}-b^{8}+\left(1+b^{2}\right)^{2} \sqrt{50 b^{4}-24 b^{6}-b^{8}-8 b^{2}-1}}(4- \\
& \left.\frac{M^{2}\left(1-b^{2}\right)^{2}}{3 \sqrt{50 b^{4}-24 b^{6}-b^{8}-8 b^{2}-1}}\right)+O\left(\delta^{2}\right), \\
& \sim 2-2(1-b)+O\left(M^{4},(1-b)^{2}, \delta^{2}\right) .
\end{aligned}
$$

We do not explicitly calculate the constant because we only use the drift function in a differentiated form where the constant is irrelevant. 


\section{Appendix B}

The turbulent pressure spectrum is given by

$$
\begin{aligned}
& \Theta_{p p} \approx \frac{12 \alpha k_{1} M \sqrt{\pi} \Gamma\left(\frac{1}{3}\right)}{55 \Gamma\left(\frac{5}{6}\right)}\left(\frac{l^{2}}{g_{2}+k_{1}^{2} l^{2}}\right)^{4 / 3}\left[2-{ }_{2} F_{1}\left(\frac{1}{3}, \frac{1}{2}, \frac{3}{2} ;-\frac{k_{1}^{2} l^{2} M^{2}}{g_{2}-k_{1}^{2} l^{2}}\right)\right]\left|n_{0}\right|^{2} \\
& +\frac{72 \alpha \sqrt{\pi} \Gamma\left(\frac{1}{3}\right)}{55 \Gamma\left(\frac{5}{6}\right)}\left(\frac{l^{2}}{g_{2}+k_{1}^{2} l^{2}}\right)^{1 / 3}\left[1-{ }_{2} F_{1}\left(\frac{1}{3}, \frac{1}{2}, \frac{3}{2} ;-\frac{k_{1}^{2} l^{2} M^{2}}{g_{2}-k_{1}^{2} l^{2}}\right)+O\left(M^{2}\right)\right]\left|n_{1}\right|^{2} \\
& +\frac{36 \alpha \pi^{3 / 2} \Gamma\left(\frac{1}{3}\right)}{55 \Gamma\left(\frac{5}{6}\right)}\left(\frac{l^{2}}{g_{2}+k_{1}^{2} l^{2}}\right)^{1 / 3}\left[{ }_{2} F_{1}\left(\frac{-2}{3}, \frac{1}{2}, 1 ;-\frac{k_{1}^{2} l^{2} M^{2}}{g_{2}-k_{1}^{2} l^{2}}\right)\right. \\
& \left.-{ }_{2} F_{1}\left(\frac{1}{3}, \frac{1}{2}, \frac{3}{2} ;-\frac{k_{1}^{2} l^{2} M^{2}}{g_{2}-k_{1}^{2} l^{2}}\right)+O\left(M^{2}\right)\right] \operatorname{Re}\left(n_{0} n_{1}^{*}\right) \\
& +\frac{4 k_{1} \alpha \sqrt{\pi} \Gamma\left(\frac{1}{3}\right)}{55 l^{2} \Gamma\left(\frac{5}{6}\right)}\left[8 k_{1}^{2} l^{2}\left|n_{4}\right|^{2} J_{1}+n_{2}^{2} J_{2}+2 n_{2}\left(\operatorname{Re}\left(n_{1} J_{3}^{*}\right)+\operatorname{Re}\left(n_{0} J_{4}^{*}\right)\right)\right. \\
& \left.+\frac{2\left|n_{3}\right|}{k_{1}}\left(\operatorname{Im}\left(n_{1} J_{5}\right)+\operatorname{Im}\left(n_{0} J_{7}\right)\right)+\frac{8 l^{2}}{k_{1}^{2}}\left|n_{5}\right|^{2} J_{6}+\frac{\left|n_{3}\right|^{2}}{k_{1}^{2}} J_{8}\right] \text {, }
\end{aligned}
$$

where

$$
\begin{aligned}
N_{1}= & \left(n_{0}+n_{1} \sqrt{k_{1}^{2} M^{2}-k_{3}^{2}}\right) \mathrm{e}^{-\mathrm{i} r \sqrt{k_{1}^{2} M^{2}-k_{3}^{2}}\left(\theta-\theta_{s}^{0}\right)}+n_{2} \cos \left[R \sqrt{k_{1}^{2} M^{2}-k_{3}^{2}}\left(\theta-\theta^{h}\right)\right] \\
& +\frac{n_{3}}{\sqrt{k_{1}^{2} M^{2}-k_{3}^{2}}} \sin \left[R \sqrt{k_{1}^{2} M^{2}-k_{3}^{2}}\left(\theta-\theta^{h}\right)\right], \\
N_{2}= & n_{4} \cos \left[R \sqrt{k_{1}^{2} M^{2}-k_{3}^{2}}\left(\theta-\theta^{h}\right)\right]+\frac{n_{5}}{\sqrt{k_{1}^{2} M^{2}-k_{3}^{2}}} \sin \left[R \sqrt{k_{1}^{2} M^{2}-k_{3}^{2}}\left(\theta-\theta^{h}\right)\right],
\end{aligned}
$$

with

$$
\begin{aligned}
n_{0} & =\frac{\mathrm{i} k_{1} C}{k^{3 / 2}}-\frac{U_{0, r}}{k^{3 / 2}}\left[\frac{\partial C}{\partial r}+\mathrm{i} k_{1} C \frac{\partial \Delta\left(\theta_{s}^{0}\right)}{\partial r}\right], & n_{1} & =\frac{\mathrm{i} C \theta}{k^{3 / 2}} U_{0, r}+\frac{\mathrm{i} C}{k^{3 / 2}} U_{0, \theta}, \\
n_{2} & =U_{0, r} \frac{F_{1}}{R} \frac{\partial}{\partial r}\left[R\left(\theta^{h}-\theta_{s}^{0}\right)\right]+\frac{F_{1}}{r} U_{0, \theta}, & n_{3} & =\mathrm{i} U_{0, r} \frac{F_{1}}{R} k_{1} \frac{\partial \Delta\left(R, \theta^{h}\right)}{\partial r}, \\
n_{4} & =U_{0, r} \frac{F_{2}}{R} \frac{\partial}{\partial r}\left[R\left(\theta^{h}-\theta_{s}^{0}\right)\right]+\frac{F_{2}}{r} U_{0, \theta}, & n_{5} & =\mathrm{i} U_{0, r} \frac{F_{2}}{R} k_{1} \frac{\partial \Delta\left(R, \theta^{h}\right)}{\partial r},
\end{aligned}
$$

and we define

$$
\begin{aligned}
\left.\boldsymbol{u}^{(I)} \cdot \boldsymbol{n}\right|_{\left(R, \theta^{h} ; r\right)} & =A_{i} F_{i}\left(R, \theta^{h} ; r\right) \mathrm{e}^{\mathrm{i} k_{1} \Delta+\mathrm{i} k_{2} \Psi+\mathrm{i} k_{3} z}, \\
\bar{\phi}^{p} & =\frac{A_{1} C\left(r, t, k_{1}\right)}{k^{3 / 2}} \mathrm{e}^{\mathrm{i} k_{1} \Delta\left(r, \theta_{s}^{0}\right)+\mathrm{i} k_{2} \Psi\left(r, \theta_{s}^{0}\right)+\mathrm{i} k_{3} z-\mathrm{i} r \sqrt{k_{1}^{2} M^{2}-k_{3}^{2}}\left(\theta-\theta_{s}^{0}\right)},
\end{aligned}
$$




$$
\boldsymbol{U}=\left(U_{0, r}, U_{0, \theta}, U_{0, z}\right)+M^{2}\left(U_{1, r}, U_{1, \theta}, U_{1, z}\right)
$$

The function ${ }_{2} F_{1}$ denotes a hypergeometric function (Abramowitz \& Stegun, 1964, p. 556). When integrating over $k_{3}$ to obtain the pressure spectra, we take only $\left|k_{3}\right| \leq M\left|k_{1}\right|$ to ensure an oscillatory solution for $\bar{\phi}$ rather than an exponentially decaying one. The velocity, $\boldsymbol{U}$, is the velocity field due to the uniform flow around an ellipse as calculated in Section 4.1. The $J_{i}$ are defined by

$$
\begin{aligned}
& J_{1}=\int_{0}^{M}\left(1+s^{2}\right)\left(\frac{l^{2}}{g_{2}+k_{1}^{2}\left(1+s^{2}\right) l^{2}}\right)^{7 / 3} \cos ^{2}\left[R k_{1} \sqrt{M^{2}-s^{2}}\left(\theta-\theta^{h}\right)\right] d s \\
& J_{2}=\int_{0}^{M}\left(\frac{l^{2}}{g_{2}+k_{1}^{2}\left(1+s^{2}\right) l^{2}}\right)^{7 / 3}\left(3 g_{2}+k_{1}^{2}\left(3+11 s^{2}\right) l^{2}\right) \cos ^{2}\left[R k_{1} \sqrt{M^{2}-s^{2}}\left(\theta-\theta^{h}\right)\right] d s
\end{aligned}
$$

$$
\begin{gathered}
J_{3}=\int_{0}^{M} \sqrt{M^{2}-s^{2}}\left(\frac{l^{2}}{g_{2}+k_{1}^{2}\left(1+s^{2}\right) l^{2}}\right)^{7 / 3}\left(3 g_{2}+k_{1}^{2}\left(3+11 s^{2}\right) l^{2}\right) \\
\cos \left[R k_{1} \sqrt{M^{2}-s^{2}}\left(\theta-\theta^{h}\right)\right] \mathrm{e}^{\mathrm{i} r k_{1} \sqrt{M^{2}-s^{2}}\left(\theta-\theta_{s}^{0}\right)} d s,
\end{gathered}
$$

$$
\begin{aligned}
& J_{4}= \int_{0}^{M}\left(\frac{l^{2}}{g_{2}+k_{1}^{2}\left(1+s^{2}\right) l^{2}}\right)^{7 / 3}\left(3 g_{2}+k_{1}^{2}\left(3+11 s^{2}\right) l^{2}\right) \mathrm{e}^{\mathrm{i} r k_{1} \sqrt{M^{2}-s^{2}}\left(\theta-\theta_{s}^{0}\right)} \\
& \cos \left[R k_{1} \sqrt{M^{2}-s^{2}}\left(\theta-\theta^{h}\right)\right] d s \\
& J_{5}= \int_{0}^{M} \frac{1}{\sqrt{M^{2}-s^{2}}}\left(\frac{l^{2}}{g_{2}+k_{1}^{2}\left(1+s^{2}\right) l^{2}}\right)^{7 / 3}\left(3 g_{2}+k_{1}^{2}\left(3+11 s^{2}\right) l^{2}\right) \\
& J_{6}= \int_{0}^{M} \frac{1+s^{2}}{M^{2}-s^{2}}\left(\frac{l^{2}}{g_{2}+k_{1}^{2}\left(1+s^{2}\right) l^{2}}\right)^{7 / 3} \sin ^{2}\left[R k_{1} \sqrt{M^{2}-s^{2}}\left(\theta-\theta^{h}\right)\right] d s \\
& J_{7}= \int_{0}^{M} \frac{1}{\sqrt{M^{2}-s^{2}}}\left(\frac{l^{2}}{g_{2}+k_{1}^{2}\left(1+s^{2}\right) l^{2}}\right)^{7 / 3}\left(3 g_{2}+k_{1}^{2} l^{2}\left(3+11 s^{2}\right)\right) \mathrm{e}^{\mathrm{i} r k_{1} \sqrt{M^{2}-s^{2}}\left(\theta-\theta_{s}^{0}\right)} \\
& J_{8}= \int_{0}^{M} \frac{1}{\sin ^{2}\left[R k_{1} \sqrt{M^{2}-s^{2}}\left(\theta-\theta^{h}\right)\right] d s}\left(\frac{l^{2}}{M_{2}+k_{1}^{2}\left(1+s^{2}\right) l^{2}}\right)^{7 / 3}\left(3 g_{2}+k_{1}^{2} l^{2}\left(3+11 s^{2}\right)\right) \\
& \sin ^{2}\left[R k_{1} \sqrt{M^{2}-s^{2}}\left(\theta-\theta^{h}\right)\right] d s
\end{aligned}
$$




\section{Chapter 5}

\section{Concluding Remarks and Further Work}

This dissertation has considered various ways in which sound is generated in an aeroengine, with a particular focus towards the sound generated by blade-blade interactions within the engine. We have constructed semi-analytic solutions for certain interactions; gust-aerofoil interaction in steady, subsonic, uniform and shear flows; and sound-aerofoil interaction in steady, subsonic uniform flow. To find these solutions we had to separate the solution domain into various asymptotic regions wherein different behaviour dominated the production of sound. The regions were matched using Van Dyke's matching rule. The unsteady pressure singularity arising on the nose of thick aerofoils during gust-aerofoil interaction in uniform flow has been accounted for by constructing a new asymptotic solution specifically close to the incident stagnation point that can be combined with the solutions considered in earlier work (where the singularity was present). This correction is currently only valid for low Mach numbers, although if certain functions could be solved for computationally (requiring only simple numerical methods, but doing so is beyond the aims of this dissertation), we could extend our solutions to the range of Mach numbers more typically seen during aircraft flight. These solutions can be implemented in parallel with computational schemes, allowing the acoustics of the aeroengine to be modelled more quickly than is currently possible.

We have identified dominant sources of far-field noise generated by sound-aerofoil interaction, which can be reduced by altering the aerofoil geometry. These sound-aerofoil interactions arise due to sound scattering upstream from gust-aerofoil interaction, hence the dominant noise could also be reduced by altering the gust-aerofoil interaction noise. We have presented a simple method of calculating the maximum noise output from sound-aerofoil interaction, hence provided with gust-aerofoil scattering data, we could calculate the maximum acoustic pressure generated by sound-aerofoil interaction. Further work is required to establish the complicated relationship between the gust-aerofoil noise, and the maximal sound-aerofoil noise.

To analyse gust-aerofoil interaction (in either uniform or shear flow) we chose a single frequency sinusoidal upstream vortical disturbance, which is typically used computation- 
ally and analytically because a Fourier composition of such gusts can generate an arbitrary upstream vortical disturbance, yet their simplicity allows for analytic solutions to be obtained without too much difficulty. Gusts of arbitrary shape have been considered by Chapman $(2002,2003)$; the leading-edge flat-plate acoustic directivity is obtained using Wiener-Hopf techniques similar to those in Chapters 2 and 3. It would therefore be possible to consider the noise generated by gust-aerofoil interaction for gusts of arbitrary shape using a similar method as outlined in Chapters 2 and 3, however, as shown by Chapman (2003), a more complicated solution for the pressure would be obtained. This complication would only magnify when considering the rescattering of the leading-edge ray field by the trailing edge. The benefit of analysing the noise generated by gusts of arbitrary shape would be to allow a wider range of results for CAA code validation. Indeed, the principal benefits of analytic solutions are to obtain results against which CAA codes can be validated, and be implemented directly into CAA codes.

We have attempted to validate our results against previous analytic and computational solutions, in particular for gust-aerofoil interaction in uniform flow. We found agreement analytically with Myers \& Kerschen (1997) and Tsai (1992) in the limiting cases of zero-thickness and zero-camber respectively. Comparison with current numerical schemes is difficult due to the lack of high-frequency solutions available; we have only been able to find one scheme (Gill et al., 2013) which permits high enough gust frequencies in moderate speed flows. It appears from our comparison with Hixon et al. (2006) that our analytic solution is viable for mid-range frequencies, however it would be difficult to assess the accuracy in this regime. As yet we are not aware of any numerical solutions for high-frequency gust-aerofoil interaction in shear flow, however we have been able to recover a result consistent with the uniform flow solution for some validation. Given the importance of the shear flow problem we are hopeful that numerical solutions may be on the horizon, and our analytic solution will prove mutually beneficial for CAA code validation. Similarly the high-frequency sound-aerofoil interaction problem has not yet been considered computationally for aerofoils with real geometry, nor has it been considered analytically.

The intuitive next step to this work would be to consider gust-cascade interaction (i.e. the sound generated by a gust interacting with a staggered cascade of generalised aerofoils) in uniform steady subsonic flow, and indeed also sound-cascade interaction. This would allow us to comprehensively assess the rotor blockage preventing sound from radiating upstream of the blade-blade system and out of the engine inlet. We must 
also investigate how the Fresnel regions propagate through a cascade, as these will likely indicate locations of maximum noise output. Blandeau et al. (2011) find that the upstream and downstream high-frequency sound power radiated by a cascade has a strong relationship with the single aerofoil result (provided the frequency is sufficiently high). Therefore the single gust-aerofoil results in Chapter 2 could prove greatly beneficial for high-frequency cascade models. However, this correlation is only valid for a restricted set of cascade parameters and it is not clear if this relationship will hold for more general cases. The sound power is obtained in Blandeau et al. (2011) by considering the response function, which is related to the pressure distribution on the aerofoil surface. It would therefore be of interest to obtain an analytic approximation for the acoustic pressure generated in both the near and far field of a cascade containing blades with real geometry; we could then compare the radiated sound power for a wider range of parameters.

We mentioned in the Introduction that the flat-plate gust-cascade problem has been analysed by Peake \& Kerschen (1997, 2004), and approximations for the upstream and downstream acoustic radiation has been found. Evers \& Peake (2002) have also considered gust-cascade interaction for generalised aerofoils, but given the complexity of the method (requiring conformal mapping from the generalised cascade to a flat-plate cascade) only the upstream radiation has been found. Applying Evers's theory to obtain approximations for the downstream radiation would result in long numerical codes to evaluate the conformal mapping and steady flow interaction throughout the cascade, before applying the results from Peake and Kerschen. We believe it would be easier to obtain an approximation for the downstream acoustic radiation by directly adapting the method from Peake \& Kerschen (2004) for the base solution of single gust-aerofoil interaction for a generalised aerofoil, therefore it is necessary to have an analytic solution for the far-field acoustic radiation generated by single gust-aerofoil interaction for a generalised aerofoil. Sound-cascade interaction for flat-plate cascades has been considered by Amiet (1971), who used matched asymptotic expansions to solve for the high-frequency noise transmitted through a blade row. Koch (1971) extended this problem, by constructing a finite Wiener-Hopf problem for the far-field sound (of any frequency) transmitted through a blade row. Whilst this is relevant to incident sound waves of any frequency, the simplicity of the blades (not only flat, but at zero angle of attack) does not yield results that accurately model the true design of an aeroengine. Even recent work by Posson et al. (2013) considers flat-plate cascades at zero angle of attack. Now that we have an asymptotic solution for the single-blade sound-aerofoil interaction problem, we 
expect that, as with gust-cascade interaction, upstream and downstream approximations for the noise generated during sound-cascade interaction could be obtained by applying the theory of Peake \& Kerschen $(1997,2004)$ to the solution obtained from single soundaerofoil interaction. This would significantly extend Amiet (1971) to yield cascade results for realistic geometries.

Finally, we mention a purely mathematical problem arising from this dissertation; in Sections 1.2.1 and 1.2.2 we encountered two integrals with singularities, and upon use of the standard method of stationary phase we obtained singular far-field approximations. We therefore had to consider the effects of the pole and square root-type branch point more carefully. The square root-type singularity (by far the more unusual of the two) arose from the boundary condition accompanying the unforced Helmholtz equation, (1.2.2.1b), and this was a direct result of the leading-edge inner approximation for the potential, (1.2.0.1). Therefore, if the nose of the aerofoil were not parabolic (e.g. $y \sim x^{1 / 3}$ rather than $x^{1 / 2}$ ), we would see a different branch point singularity arise in the expression for $H_{1}$ given in (1.2.2.2). Indeed, one could go further and consider the effects of a general singularity interacting with a general saddle point of arbitrary order (not just the simple saddle points obtained from the Helmholtz equation) to obtain uniformly-valid expressions for highly oscillatory integrals with singularities. Work on this topic has been considered by Bleistein \& Handelsman (1975), however current results do not generalise the order of the point of stationary phase, or the branch cut singularity. Bleistein \& Handelsman (1975) also highlight other integral problems during which there is interference between points of stationary phase and the standard methods cannot be used. Further work to make uniform expressions in these singular cases looks most promising. 


\section{Bibliography}

Abbott, I. H. \& Von Doenhoff, A. E. 1959 Theory of Wing Sections, Including a Summary of Airfoil Data. Courier Dover Publications.

Ablowitz, M. J. \& FokAs, A. S. 2003 Complex Variables: Introduction and Applications. Cambridge University Press.

Abramowitz, M. \& Stegun, I. A. 1964 Handbook of Mathematical Functions: With Formulas, Graphs, and Mathematical Tables. Courier Dover Publications.

Agarwal, A., Dowling, A. P., Shin, H-C., Graham, W. \& Sefi, S. 2007 Ray tracing approach to calculate acoustic shielding by a flying wing airframe. AIAA Journal 45, 1080-1090.

Allampalli, V., Hixon, R., Nallasamy, M. \& Sawyer, S. D. 2009 High-accuracy large-step explicit Runge-Kutta (HALE-RK) schemes for computational aeroacoustics. Journal of Computational Physics 228, 3837-3850.

Amiet, R.K. 1975 Acoustic radiation from an airfoil in a turbulent stream. Journal of Sound and Vibration 41, 407-420.

Amiet, R.K. 1978 Refraction of sound by a shear layer. Journal of Sound and Vibration 58, 467-482.

Amiet, R. K. 1971 Transmission and reflection of sound by a blade row. AIAA Journal pp. $71-181$.

AtAssi, H. M. 1984 The Sears problem for a lifting airfoil revisited - new results. Journal of Fluid Mechanics 141, 109-122.

Atassi, H. M., FAng, J. \& PATrick, S. 1993 Direct calculation of sound radiated from bodies in nonuniform flows. Journal of Fluids Engineering 115 (4), 573.

Ayton, L. J. \& PeAke, N. 2013 On high-frequency noise scattering by aerofoils in flow. Journal of Fluid Mechanics 734, 144-182.

Ayton, L. J. \& PeAke, N. 2014a An analytic approach to high-frequency gust-aerofoil interaction noise in steady shear flows. 20th AIAA/CEAS Aeroacoustics Conference, Atlanta 2014-2322. 
Ayton, L. J. \& PeAke, N. $2014 b$ On high-frequency sound generated by gust-aerofoil interaction in shear flow (under consideration). Journal of Fluid Mechanics .

Batchelor, G. K. 1967 An Introduction to Fluid Dynamics. Cambridge University Press.

Bender, C. M. \& Orszag, S. A. 1978 Advanced Mathematical Methods for Scientists and Engineers. Springer.

Blandeau, V.P., Joseph, P.F., Jenkins, G. \& Powles, C.J 2011 Comparison of sound power radiation from isolated airfoils and cascades in a turbulent flow. Journal of the Acoustical Society of America 129, 3521-3530.

Bleistein, N. \& Handelsman, R. A. 1975 Asymptotic Expansions of Integrals. Ardent Media.

Chapman, C.J. 2002 High-speed leading-edge noise. Proceedings of the Royal Society A: Mathematical, Physical and Engineering Sciences 459, 2131-2151.

Chapman, C.J. 2003 Some benchmark problems for computational aeroacoustics. Journal of Sound and Vibration 270, 495-508.

Crighton, D. G. 1985 The Kutta condition in unsteady flow. Annual Review of Fluid Mechanics 17, 411-445.

Durbin, P. A. 1978 Rapid Distortion Theory of Turbulent Flows. PhD thesis, University of Cambridge.

Durbin, P. A. 1983 High frequency Green function for aerodynamic noise in moving media, Part I: General theory. Journal of Sound and Vibration 91, 519-525.

Durbin, P. A. \& Pettersson Reif, B. A. 2001 Statistical Theory and Modeling for Turbulent Flows. Wiley.

Evers, I. \& PEAKE, N. 2002 On sound generation by the interaction between turbulence and a cascade of airfoils with non-uniform mean flow. Journal of Fluid Mechanics 463, $25-52$.

Ffowcs Williams, J. E. \& Hawkings, D. L. 1969 Sound generation by turbulence and surfaces in arbitrary motion. Philosophical Transactions of the Royal Society A 264, 321-342. 
FinnigAn, J. J. 1983 A streamline coordinate system for distorted two-dimensional shear flows. Journal of Fluid Mechanics 130, 241-258.

GershfelD, J. 2004 Leading edge noise from thick foils in turbulent flows. Journal of the Acoustical Society of America 116, 1416-1426.

Geyer, T., Sarradj, E. \& Giesler, J. 2012 Application of a beamforming technique to the measurement of airfoil leading edge noise. Advances in Acoustics and Vibration 2012, 1-16.

Gill, J., Zhang, X., Joseph, P. F. \& Node-Langlois, T. 2013 Effects of real airfoil geometry on leading edge gust interaction noise. 19th AIAA/CEAS Aeroacoustics Conference, Berlin, DE.

Goldstein, M. E. 1976 Aeroacoustics. McGraw-Hill.

Goldstein, M. E. 1978a Characteristics of the unsteady motion on transversely sheared mean flows. Journal of Fluid Mechanics 84, 305-329.

Goldstein, M. E. $1978 b$ Unsteady vortical and entropic distortions of potential flows round arbitrary obstacles. Journal of Fluid Mechanics 89, 433-468.

Goldstein, M. E. 1979 Scattering and distortion of the unsteady motion on transversely sheared mean flows. Journal of Fluid Mechanics 91, 601-632.

Goldstein, M. E., Afsar, M. Z. \& Leib, S. J. 2013 Non-homogeneous rapid distortion theory on transversely sheared mean flows. Journal of Fluid Mechanics 736, $532-569$.

Goldstein, M. E. \& AtAssi, H. 1976 A complete second-order theory for the unsteady flow about an airfoil due to a periodic gust. Journal of Fluid Mechanics 74, 741-765.

Gradshteyn, I. S. \& Ryzhik, I. M. 1980 Table of Integrals, Series, and Products, sixth edn. Academic Press.

Hixon, R., Scott, J. R., Sawyer, S. \& Nallasamy, M. 2006 Application of a nonlinear computational aeroacoustics code to the gust-airfoil problem. AIAA Journal 44, 323-328.

Howe, M. S. 1978 A review of the theory of trailing edge noise. Contractor Report Jul Dec 1977 Bolt Beranek and Newman Inc Cambridge MA 61, 437-465. 
Hunt, J. C. R. 1973 A theory of turbulent flow round two-dimensional bluff bodies. Journal of Fluid Mechanics 61, 625-706.

Jones, D. S. 1966 Generalised Functions. McGraw-Hill.

Jones, D. S. 1986 Acoustic and Electromagnetic Waves. Clarendon Press.

Kerschen, E. J. \& BAlsA, T. F. 1981 Transformation of the equation governing disturbances of a two-dimensional compressible flow. AIAA Journal 19, 1367-1370.

Kerschen, E. J. \& Myers, M. R. 1987 Perfect gas effects in compressible rapid distortion theory. AIAA Journal 25, 504-507.

Koch, W. 1971 On the transmission of sound waves through a blade row. Journal of Sound and Vibration 18, 111-128.

Kolmogorov, A. N. 1941 The local structure of turbulence in incompressible viscous fluid for very large Reynolds numbers. Doklady Akademiia Nauk SSSR 30, 301-305.

Lighthill, M J 1956 Drift. Journal of Fluid Mechanics 1, 31-53.

Mish, P. F. 2001 Mean Loading and Turbulence Scale Effects on the Surface Pressure Fluctuations Occurring on a NACA 0015 Airfoil Immersed in grid Generated Turbulence. PhD thesis, Virginia Polytechnic Institute and State University.

Mish, P. F. \& Devenport, W. J. 2003 An experimental investigation of unsteady surface pressure on an airfoil in turbulence. Tech. Rep.. Final report to NASA Langley.

Myers, M. R. 1987 Effect of airfoil mean loading on high-frequency gust interaction noise. PhD thesis, University of Arizona.

Myers, M. R. \& Kerschen, E. J. 1995 Influence of incidence angle on sound generation by airfoils interacting with high-frequency gusts. Journal of Fluid Mechanics 292, 271-304.

Myers, M. R. \& Kerschen, E. J. 1997 Influence of camber on sound generation by airfoils interacting with high-frequency gusts. Journal of Fluid Mechanics 353, 221-259.

NoBle, B. 1998 Methods Based on the Wiener-Hopf Technique for the Solution of Partial Differential Equations. Chelsea Publications. 
Peake, N. \& Kerschen, E. J. 1997 Influence of mean loading on noise generated by the interaction of gusts with a flat-plate cascade: upstream radiation. Journal of Fluid Mechanics 347, 315-346.

Peake, N. \& Kerschen, E. J. 2004 Influence of mean loading on noise generated by the interaction of gusts with a cascade: downstream radiation. Journal of Fluid Mechanics 515, 99-133.

PeAke, N. \& PARry, A. B. 2012 Modern challenges facing turbomachinery aeroacoustics. Annual Review of Fluid Mechanics 44, 227-248.

Posson, H., Bériot, H. \& Moreau, S. 2013 On the use of an analytical cascade response function to predict sound transmission through an annular cascade. Journal of Sound and Vibration 332, 3706-3739.

Sakurai, A. \& Arai, T. 1981 Solution of Imai's equation for compressible flow past a cylinder. Journal of the Physics Society Japan 50, 3782-3784.

ScotT, J. R. 2004 Benchmark solutions for computational aeroacoustics (CAA) code validation. NASA Technical Report 2004, NASA/TM-2004-213386.

Scott, J. R. \& AtAssi, H. M. 1995 A Finite-Difference, Frequency-Domain Numerical Scheme for the Solution of the Gust Response Problem. Journal of Computational Physics 119 (1), 75-93.

SEARS, W. R. 1941 Some aspects of non-stationary airfoil theory and its practical applications. Journal of the Aeronautical Sciences 8, 104-188.

SowYRDA, A. 1958 Theory of cambered Joukowsky airfoils in shear flow. Tech. Rep.. Cornell University.

THWAites, B. 1960 Incompressible aerodynamics: an account of the theory and observation of the steady flow of incompressible fluid past aerofoils, wings, and other bodies. Dover Publications.

TsAi, C-T. 1992 Effect of Airfoil Thickness on High-Frequency Gust Interaction Noise. $\mathrm{PhD}$ thesis, University of Arizona.

VAN Der Waerden, B. L. 1952 On the method of saddle points. Applied Scientific Research, Section B 2, 33-45.

VAn Dyke, M. 1975 Perturbation methods in fluid mechanics. Parabolic Press. 